\title{
Structural and Mechanical Properties of Silica and Hybrid Aerogels and Xerogels Studied Using Molecular Dynamics Simulations
}

John Sandro Rivas Murillo

West Virginia University

Follow this and additional works at: https://researchrepository.wvu.edu/etd

\section{Recommended Citation}

Rivas Murillo, John Sandro, "Structural and Mechanical Properties of Silica and Hybrid Aerogels and Xerogels Studied Using Molecular Dynamics Simulations" (2012). Graduate Theses, Dissertations, and Problem Reports. 3604.

https://researchrepository.wvu.edu/etd/3604

This Dissertation is protected by copyright and/or related rights. It has been brought to you by the The Research Repository @ WVU with permission from the rights-holder(s). You are free to use this Dissertation in any way that is permitted by the copyright and related rights legislation that applies to your use. For other uses you must obtain permission from the rights-holder(s) directly, unless additional rights are indicated by a Creative Commons license in the record and/ or on the work itself. This Dissertation has been accepted for inclusion in WVU Graduate Theses, Dissertations, and Problem Reports collection by an authorized administrator of The Research Repository @ WVU.

For more information, please contact researchrepository@mail.wvu.edu. 


\title{
Structural and Mechanical Properties of Silica and Hybrid Aerogels and Xerogels Studied Using Molecular Dynamics Simulations
}

\author{
John Sandro Rivas Murillo \\ Dissertation submitted to the \\ College of Engineering and Mineral Resources \\ at West Virginia University \\ in partial fulfillment of the requirements \\ for the degree of \\ Doctor of Philosophy \\ in \\ Mechanical Engineering \\ Committee Members
}

Ever J. Barbero, Ph.D. (Chairperson and Advisor)

Martina E. Bachlechner, Ph.D.

Darran Cairns, Ph.D.

Edward Sabolsky, Ph.D.

Nianqiang Wu, Ph.D.

Department of Mechanical and Aerospace Engineering

Morgantown, WV, USA

2012

Keywords: Classical Molecular Dynamics Simulations; Computational Materials; Silica Aerogel; Silica Xerogels; Hybrid Aerogel; Hybrid Xerogels; Structural Properties; Mechanical Properties 


\section{Abstract}

\section{Structural and Mechanical Properties of Silica and Hybrid Aerogels and Xerogels Studied Using Molecular Dynamics Simulations}

\section{John Sandro Rivas Murillo}

The structural and mechanical properties of silica and hybrid aerogels and xerogels are studied using Molecular Dynamics simulations. Hybrid samples are created by adding methyl groups $\left(\mathrm{CH}_{3}\right)$ to the silica structure. Silica samples are generated expanding a dense silica glass in a simulation box of volume chosen to produce a porous sample of a specified density. The expanded glass is thermally treated using a combination of heating and cooling processes. The heating process increases the kinetic energy of the atoms in the system, which facilitates their movement throughout the system. The cooling process reduces the kinetic energy of the atoms and helps locking them in place forming the desired porous structure. A similar procedure is used to create the hybrid samples, but $\mathrm{CH}_{3}-\mathrm{SiO}_{2}$ molecules are randomly inserted in the expanded structure before the thermal treatment of the sample.

The structures are characterized estimating the fractal dimension of the samples. It is calculated using its relationship with the decay of the Pair Distribution Function of the samples in the intermediate range of structural arrangement. The fractal dimension of the samples used for this study compares favorably to the values found reported in experimental studies, which validates the preparation processes used here. The mechanical properties of the samples are obtained by modeling a tension test performed by stretching the sample along one direction. The stress vs. strain relationship is estimated for all the samples. The results reproduce trends similar to those reported from physical experiments.

The computational models show that the addition of methyl groups reduces the stiffness of the structure allowing it to stretch more easily, and increasing the toughness of the samples. From the stress-strain plots is concluded that as the amount of methyl groups in the structure is increased also does the compliance of the sample, effectively making the nature of the material similar to a rubber-like material. These results are in agreement with physical experimental findings which show that adding methyl groups to silica allows compressing the samples more than $50 \%$ without destroying them. 
To my family, my life support.

My father Jose Catalino, my mother Maria Leida, my brothers Oscar, Alirio, Carlos Ivan, and Jose Antonio, my sisters Lina Maria and Julia Ema, and to my nephews and nieces, especially Andrea and Alejandra. Thanks for always being there for me!. 


\section{Acknowledgments}

I have had the honor of working with the guidance and support of:

Dr. Ever J. Barbero, my academic advisor, an excellent scientist and a great human being. He always helped me to find the right approach to solve my research problems. He is my academic father. And Dr. Martina E. Bachlechner, who thought me all I needed to understand the Molecular Dynamics technique, and gave me much needed advise whenever I struggled; definitively, my academic mother.

Big thanks to the faculty and staff of the Mechanical \& Aerospace Department of West Virginia University for the opportunity the gave me to learn and to teach in a very constructive environment.

And to all my dear friends, a fundamental part of my life.

I would like to thank so many people that helped me and made my life happy during all this time at WVU. Unfortunately I can not list all of them here; but I hope they know how grateful I am.

Let's go Mountaineers! 


\section{Contents}

\begin{tabular}{|ll} 
Abstract & ii
\end{tabular}

\begin{tabular}{|l|l}
\hline Table of Contents & v
\end{tabular}

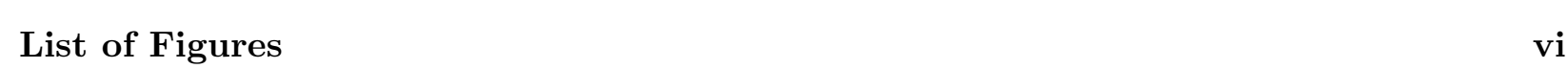

List of Tables $\quad$ ix

1 Silica and Hybrid Aerogels and Xerogels Materials 1

1.1 Aerogel and Xerogel Materials . . . . . . . . . . . . . . . . . 2

1.2 Silica Aerogels and Xerogels . . . . . . . . . . . . . . . . . . . 5

1.2.1 Structure of Silica Aerogels and Xerogels . . . . . . . . . . . . . . . 5

1.2.2 Mechanical Properties of Aerogels and Xerogels . . . . . . . . . . . . . . . 8

1.3 Hybrid Aerogels and Hybrid Xerogels _. . . . . . . . . . . . . . . . . 9

1.3.1 Structure of $\mathrm{CH}_{3}$-Added Silica Aerogels and Xerogels . . . . . . . . . . . 10

1.3.2 Mechanical Properties of $\mathrm{CH}_{3}$-Added Silica Aerogels and Xerogels . . . . . . 12

2 Molecular Dynamics Simulation Technique 14

2.1 Molecular Dvnamics Simulation Technique . . . . . . . . . . . . . . . . . . . . . . . . . . . . . . 15

2.1 .1 Statistical Ensembles . . . . . . . . . . . . . . . . . . . . 16

2.1 .2 Interatomic Potential _. . . . . . . . . . . . . . . 17

2.1.3 Initial and Boundarv Conditions . . . . . . . . . . . . . . . 18

2.1.4 Integration Algorithm for the Equations of Motion . . . . . . . . . . . . . 19

2.1.5 Molecular Dvnamics of Constrained Systems . . . . . . . . . . . . . . 20

2.2 Phvsical Properties of Atomic Svstems . . . . . . . . . . . . . . . . . 22

2.2.1 Temperature and Kinetic Energy of an Atomic System . . . . . . . . . . . . 22

2.2 .2 Pressure in an Atomic Svstem . . . . . . . . . . . . . . . . . . . . . . . . . . . . . . . . . . 22

2.2 .3 Stress in Atomic Svstems . . . . . . . . . . . . . . . . . . . 24

2.2 .4 Structural Properties of Atomic Systems . . . . . . . . . . . . . . . . 25

3 Molecular Dynamics Simulation Models for Silica Aerogels and Xerogels 28

3.1 MD Models of the Structure of Silica Aerogels and Xerogels . . . . . . . . . . . . . 29 
3.2 MD Models of the Mechanical Properties of Silica Aerogels and Xerogels . . . . . . . 30

3.3 Interatomic Potential for Silica $\ldots \ldots \ldots \ldots$. . . . . . . . . . . 31

3.4 MD Model of Dense Silica Glassed . . . . . . . . . . . . . . . . . . . 34

3.4.1 Sample Preparation and Structural Characterization . . . . . . . . . . . 34

3.4.2 Mechanical Characterization . . . . . . . . . . . . . . . . 40

3.5 MD Model of Silica Aerogels and Xerogels . . . . . . . . . . . . . . . . . . . . 44

3.5.1 Sample Preparation . . . . . . . . . . . . . . . . . . . . . . . . . . . . . . . . 45

3.5.2 Structural Characterization . . . . . . . . . . . . . . . 46

3.5.3 Mechanical Characterization . . . . . . . . . . . . . . . . 49

4 Molecular Dynamics Simulation Models for Hybrid Aerogels and Xerogels $\quad 57$

4.1 Interatomic Potentials for $\mathrm{CH}_{3}$-Added Systems $\ldots \ldots \ldots \ldots$. . . . . . . . 58

4.2 Preparation of $\mathrm{CH}_{3}$-Added Svstems . . . . . . . . . . . . . . . . . 60

4.2.1 Constraint Dvnamics applied to $\mathrm{CH}_{3}-\mathrm{SiO}_{2}$ molecules . . . . . . . . . . . 64

4.3 Structural Characterization of $\mathrm{CH}_{3}$-Added Svstems . . . . . . . . . . . . . . . . . . . . . . . .

4.4 Mechanical Characterization of $\mathrm{CH}_{3}$-Added Svstems $\ldots \ldots \ldots \ldots$. . . . . . . 72

$\begin{array}{lll}5 \text { Conclusions } & 80\end{array}$

$\begin{array}{ll}\text { Bibliography } & 90\end{array}$

$\begin{array}{ll}\text { A Additional Figures: Samples } & 91\end{array}$

B Additional Figures: Pair Distribution Function \& Fractal Dimension 104

$\begin{array}{ll}\text { C Additional Figures: Stress vs. Strain } & 117\end{array}$ 


\section{List of Figures}

1.1 Schematic of the process to make an aerogel . . . . . . . . . . . . . . . 5

1.2 Dependence of the densitv of a porous svstem on the scale length . . . . . . . . . 7

1.3 Molecules formed bv hvdrolvsis and polvmerization of MTMS . . . . . . . . . 11

1.4 Molecules formed by hydrolysis and polymerization of TMOS:TRIMOS mixtures . . 12

2.1 Example of the Pair Distribution Function for a silica aerogel . . . . . . . . . . . 26

2.2 Example of scattering intensity vs. wavenumber plot for a silica aerogel . . . . . . 27

3.1 Temperature evolution during the creation of a sample of amorphous silica. . . . . . 35

3.2 Total energy per particle during the creation of a sample of amorphous silica _ . . . 36

3.3 PDF of Si-O distances for $\beta$-cristobalite and amorphous silica at $300 \mathrm{~K}$ and $0 \mathrm{~K}$. . 37

3.4 PDF of Si-Si distances for amorphous silica at $300 \mathrm{~K}$ and $0 \mathrm{~K} \ldots \ldots$. . . . . . . 37

$3.5 \mathrm{PDF}$ of $\mathrm{O}-\mathrm{O}$ distances for amorphous silica at $300 \mathrm{~K}$ and $0 \mathrm{~K} \ldots \ldots . \ldots 38$

3.6 Snap-shoot of simulated silica samples . . . . . . . . . . . . . . . . . . 38

3.7 Si-O-Si angle distribution for amorphous silica at 300 and $0 \mathrm{~K} \ldots \ldots$. . . . . . 39

3.8 O-Si-O angle distribution for amorphous silica at 300 and $0 \mathrm{~K} \ldots \ldots \ldots$

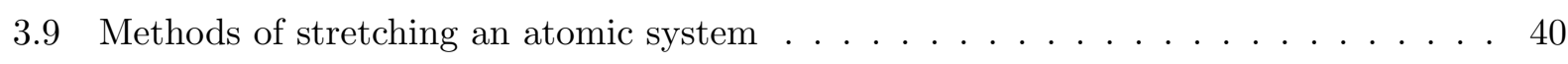

3.10 Comparison of tension tests simulated by different stretching procedures . . . . . . . 41

3.11 Stress-Strain plot for a silica glass sample stretched at different strain rates . . . . 42

3.12 Effect of the damping coefficient in the Langevin equation on simulated tension tets 43

3.13 Comparison of simulated tension test with and without temperature control . . . . . 44

3.14 Effect of the size of the system on simulated tension tests . . . . . . . . . . . 45

3.15 Samples generated for the studv of the properties of silica aerogels and xerogels . . . 47

$3.16 \mathrm{PDF}$ for samples of silica aerogels and xerogels . . . . . . . . . . . . 48

$3.17 \mathrm{PDF}$ in the intermediate range for samples of silica aerogels and xerogels . . . . . . . 49

3.18 O-Si-O angel distribution for samples of silica aerogels and xerogels . . . . . . . . . 50

$3.19 \mathrm{Si}-\mathrm{O}-\mathrm{Si}$ angel distribution for samples of silica aerogels and xerogels . . . . . . . . . 51

3.20 Scattering profiles of the samples of silica aerogels and xerogels . . . . . . . . . 52

3.21 Fractal dimension of aerogel and xerogel samples . . . . . . . . . . . . . . . 52

3.22 Stress vs. Strain plots of the samples of silica aerogels and xerogels . . . . . . . . . . 55

3.23 Sample of silica aerogel, $\rho=0.45 \frac{\mathrm{g}}{\mathrm{em}^{3}}$ before and after a tension test . . . . . . . 56 
3.24 Elastic modulus vs. densitv for silica aerogels . . . . . . . . . . . . . . 56

3.25 Strength vs. density for silica aerogels $\ldots \ldots \ldots \ldots \ldots$

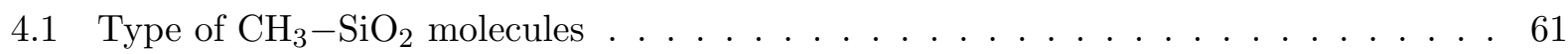

4.2 Expanded glass sample with $\mathrm{CH}_{3}-\mathrm{SiO}_{2}$ molecules added $\ldots \ldots \ldots \ldots \ldots \ldots$. . . 62

4.3 Temperature profile used to create silica and hvbrid aerogel and xerogel samples . . 64

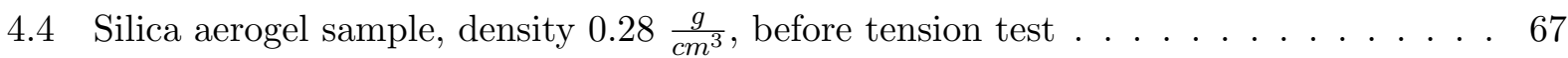

4.5 Aerogel sample with $0.1 \mathrm{~mol} \% \mathrm{CH}_{3}$ content, densitv $0.28 \frac{\mathrm{g}}{\mathrm{m} \mathrm{w}^{3}}$. before tension test . . 68

4.6 Aerogel sample with $0.4 \mathrm{~mol} \% \mathrm{CH}_{3}$ content, density $0.28 \frac{\mathrm{g}}{\mathrm{m}^{2}}$, before tension test . . 68

$4.7 \mathrm{PDF}$ and $\mathrm{df}$ for silica aerogel. $\rho=0.45 \frac{g}{67^{3}} \ldots \ldots \ldots \ldots \ldots \ldots$

4.8 PDF and $\mathrm{df}$ for hybrid aerogel, $\rho=0.45 \frac{\mathrm{g}}{2}, 0.1 \mathrm{~mol} \% \mathrm{CH}_{3}$ bonded to $\mathrm{Si} \ldots 70$

4.9 PDF and df for hybrid aerogel, $\rho=0.45 \frac{\mathrm{g}}{3}, 0.1 \mathrm{~mol} \% \mathrm{CH}_{3}$ bonded to $\mathrm{O} \ldots \ldots$

4.10 PDF hybrid aerogel, $\rho=0.45 \frac{g}{2}, 0.4 \mathrm{~mol}_{0} \mathrm{CH}_{3}$ content . . . . . . . . . 71

4.11 Fractal dimension vs. densitv plot for silica aerogels and xerogels . . . . . . . . . 71

4.12 Silica aerogel sample, densitv $0.28 \frac{g}{n^{3}}$. after tension test . . . . . . . . . . . 72

4.13 Aerogel sample with $0.1 \mathrm{~mol} \% \mathrm{CH}_{3}$ content densitv $0.28 \frac{g}{6 m^{3}}$. after tension test . . 73

4.14 Aerogel sample with $0.4 \mathrm{~mol}_{\%} \mathrm{CH}_{3}$ content. densitv $0.28 \frac{g}{\mathrm{~m}}$. after tension test . . . 73

4.15 Stress vs. Strain plot for silica and hvbrid aerogel, densitv $0.77 \frac{g}{m m^{3}} \ldots \ldots \ldots$. . . 75

4.16 Toughness vs. densitv for silica and hvbrid aerogels and xerogels . . . . . . . . 76

4.17 Elastic mod. vs. densitv plot for silica aerogels and xerogels . . . . . . . . . . . 77

4.18 Elastic mod. vs. densitv plot for hybrid aerogels and xerogels. $0.1 \mathrm{~mol} \% \mathrm{CH}_{3}$ content 77

4.19 Elastic mod. vs. densitv plot for hvbrid aerogels and xerogels. $0.4 \mathrm{~mol} \% \mathrm{CH}_{3}$ content 78

4.20 Strength vs. density plot for silica aerogels and xerogels . . . . . . . . . . 78

4.21 Strength vs. densitv plot for hvbrid aerogels and xerogels, $0.1 \mathrm{~mol} \% \mathrm{CH}_{3}$ content . . 79

4.22 Strength vs. density plot for hybrid aerogels and xerogels, $0.4 \mathrm{~mol} \% \mathrm{CH}_{3}$ content . . 79

A.1 Silica aerogel and xerogel samples before and after tension test (Pure silica study) . 92

A.2 Silica aerogel samples before and after tension test (Pure silica studv) . . . . . . . . 93

A.3 Silica aerogel and xerogel samples before tension test $\left(\mathrm{CH}_{3}\right.$-added silica studv $)$. . . 94

A.4 Silica aerogel and xerogel samples after tension test $\left(\mathrm{CH}_{3}\right.$-added silica studv $) \ldots .95$

A.5 Hvbrid aerogel and xerogel samples before tension test. $0.1 \mathrm{~mol} \% \mathrm{CH}_{3}$ bonded to $\mathrm{Si} \quad 96$

A.6 Hvbrid aerogel and xerogel samples after tension test. $0.1 \mathrm{~mol} \% \mathrm{CH}_{3}$ bonded to $\mathrm{Si}$. 97

A.7 Hybrid aerogel and xerogel samples before tension test. $0.1 \mathrm{~mol}^{2} \mathrm{CH}_{3}$ bonded to $\mathrm{O} \quad 98$

A.8 Hybrid aerogel and xerogel samples after tension test. $0.1 \mathrm{~mol} \% \mathrm{CH}_{3}$ bonded to $\mathrm{O}$. 99

A.9 Hybrid aerogel and xerogel samples before tension test, $0.4 \mathrm{~mol} \% \mathrm{CH}_{3}$ bonded to $\mathrm{Si} 100$ A.10 Hvbrid aerogel and xerogel samples after tension test. $0.4 \mathrm{~mol}_{\%} \mathrm{CH}_{3}$ bonded to $\mathrm{Si} .101$

A.11 Hvbrid aerogel and xerogel samples before tension test. $0.4 \mathrm{~mol}_{\%} \mathrm{CH}_{3}$ bonded to $\mathrm{O} 102$

A.12 Hybrid aerogel and xerogel samples after tension test, $0.4 \mathrm{~mol}_{\%} \mathrm{CH}_{3}$ bonded to $\mathrm{O} .103$

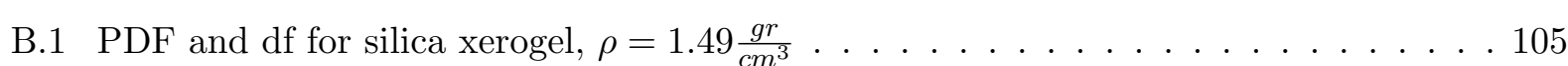


B.2 PDF and df for hybrid xerogel. $\rho=1.49 \frac{g r}{r^{2}} .0 .1 \mathrm{~mol} \% \mathrm{CH}_{3}$ bonded to $\mathrm{Si} \ldots 105$ B.3 PDF and df for hybrid xerogel. $\rho=1.49 \frac{g r}{r^{2}} .0 .1 \mathrm{~mol} \% \mathrm{CH}_{3}$ bonded to $\mathrm{O} \ldots 106$ B.4 PDF hybrid aerogel, $\rho=1.49 \frac{g}{2}, 0.4$ mol\% $\mathrm{CH}_{3}$ content $\ldots \ldots \ldots \ldots$

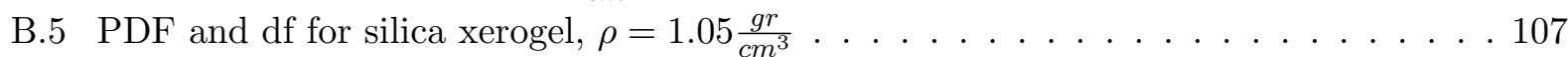

B.6 PDF and df for hybrid xerogel, $\rho=1.05 \frac{g r}{r^{3}}, 0.1 \mathrm{~mol} \% \mathrm{CH}_{3}$ bonded to $\mathrm{Si} \ldots 107$ B.7 PDF and df for hybrid xerogel, $\rho=1.05 \frac{\mathrm{gr}}{\mathrm{rr}^{2}}, 0.1 \mathrm{~mol} \% \mathrm{CH}_{3}$ bonded to $\mathrm{O} \ldots 108$

B.8 PDF hvbrid aerogel. $\rho=1.05 \frac{g}{2} .0 .4 \mathrm{~mol} \% \mathrm{CH}_{3}$ content . . . . . . . . . 108 B.9 PDF and $\mathrm{df}$ for silica aerogel. $\rho=0.77 \frac{g r}{2} \ldots \ldots \ldots \ldots \ldots \ldots \ldots$

B.10 PDF and df for hybrid xerogel. $\rho=0.77 \frac{g r}{r^{2}} .0 .1 \mathrm{~mol} \% \mathrm{CH}_{3}$ bonded to $\mathrm{Si} \ldots 109$ B.11 PDF and df for hybrid xerogel. $\rho=0.77 \frac{g r}{v^{3}}, 0.1 \mathrm{~mol} \% \mathrm{CH}_{3}$ bonded to $\mathrm{O} \ldots 110$

B.12 PDF hvbrid aerogel. $\rho=0.77 \frac{g}{2} .0 .4$ mol\% $\mathrm{CH}_{3}$ content . . . . . . . . . . 110 B.13 PDF and $\mathrm{df}$ for silica aerogel. $\rho=0.58 \frac{g r}{2 r} \ldots \ldots \ldots \ldots \ldots \ldots \ldots$

B.14 PDF and df for hybrid xerogel. $\rho=0.58 \frac{g r}{r^{3}} .0 .1 \mathrm{~mol} \% \mathrm{CH}_{3}$ bonded to $\mathrm{Si} \ldots \ldots 11$ B.15 PDF and $\mathrm{df}$ for hybrid xerogel. $\rho=0.58 \frac{\mathrm{gr}}{\mathrm{r}^{2}} .0 .1 \mathrm{~mol} \% \mathrm{CH}_{3}$ bonded to $\mathrm{O} \ldots 112$ B.16 PDF hybrid aerogel. $\rho=0.58 \frac{g}{2} \cdot 0.4 \mathrm{~mol}_{0} \mathrm{CH}_{3}$ content . . . . . . . . 112 B.17 PDF and df for silica aerogel. $\rho=0.35 \frac{g r}{2 r} \ldots \ldots \ldots \ldots \ldots \ldots \ldots \ldots$

B.18 PDF and df for hvbrid xerogel. $\rho=0.35 \frac{g r}{r^{3}} .0 .1 \mathrm{~mol} \% \mathrm{CH}_{3}$ bonded to $\mathrm{Si} \ldots 113$ B.19 PDF and df for hybrid xerogel. $\rho=0.35 \frac{g r}{v^{3}}, 0.1 \mathrm{~mol} \% \mathrm{CH}_{3}$ bonded to $\mathrm{O} \ldots \ldots 114$ B.20 PDF hybrid aerogel, $\rho=0.35 \frac{g}{2}, 0.4 \mathrm{~mol} \% \mathrm{CH}_{3}$ content . . . . . . . . . 114

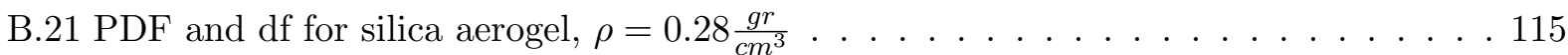

B.22 PDF and df for hybrid xerogel, $\rho=0.28 \frac{g r}{r^{2}}, 0.1 \mathrm{~mol} \% \mathrm{CH}_{3}$ bonded to $\mathrm{Si} \ldots 115$ B.23 PDF and df for hybrid xerogel. $\rho=0.28 \frac{g r}{3} .0 .1 \mathrm{~mol} \% \mathrm{CH}_{3}$ bonded to $\mathrm{O} \ldots 116$ B.24 PDF hybrid aerogel, $\rho=0.28 \frac{g}{c m m^{3}}, 0.4 \mathrm{~mol} \% \mathrm{CH}_{3}$ content . . . . . . . . . 116

C.1 Stress vs. Strain plots for silica aerogels and xerogels . . . . . . . . . . . . . 118

C.2 Stress vs. Strain plots for hybrid aerogels and xerogels, $0.1 \mathrm{~mol} \% \mathrm{CH}_{3}$ bonded to Si 119

C.3 Stress vs. Strain plots for hybrid aerogels and xerogels, $0.1 \mathrm{~mol} \% \mathrm{CH}_{3}$ bonded to $\mathrm{O} 120$

$\begin{array}{lll}\text { C. } 4 \text { Stress vs. Strain plots for hybrid aerogels and xerogels, } 0.4 \mathrm{~mol} \% \mathrm{CH}_{3} \text { bonded to } \mathrm{Si} & 121\end{array}$

C.5 Stress vs. Strain plots for hybrid aerogels and xerogels, $0.4 \mathrm{~mol} \% \mathrm{CH}_{3}$ bonded to $\mathrm{O} 122$ 


\section{List of Tables}

1.1 Exponent for the relation between elastic modulus and densitv of porous silica . . . 9

1.2 Exponent for the relation between strength and densitv of porous silica $\ldots . . . .99$

1.3 Type of molecules formed by condensation of TMOS:TRIMOS mixtures . . . . . . 12

1.4 Elastic modulus and Hardness of samples prepared from TMOS:TRIMOS mixtures . 12

3.1 Elastic modulus and strength of silica glass obtained using different potentials . . . . 32

3.2 Elastic modulus and strength for dense silica modeled with different svstem sizes . . 44

3.3 Elastic moduli and strength of silica aerogels and xerogels modeled by MD . . . . 54

3.4 Exponent for the relationship between Elastic modulus and densitv of porous silica . 54

3.5 Exponent for the relationship between strength and density of porous silica . . . . . 54

4.1 Interatomic potentials used for $\mathrm{CH}_{3}$-added svstems . . . . . . . . . . . . . 60

4.2 Number of atoms used to create silica and hybrid samples . . . . . . . . . . . 61

4.3 Density and volume of silica and hybrid aerogel and xerogel samples . . . . . . . . 63 


\section{Chapter 1}

\section{Silica and Hybrid Aerogels and Xerogels Materials}

In this chapter the descriptions of aerogel and xerogel materials in general, and silica and $\mathrm{CH}_{3}$-added silica in particular, are presented. It starts with an overview of the most important properties of this kind of materials and their potential applications, followed by a brief summary of the historical development of the processing conditions used to fabricate them. Section 1.1 presents a review of the experimental techniques used to characterize the structural features and mechanical properties of silica aerogels and xerogels. In section 1.2.1 it is highlighted that aerogels and xerogels have fractal structures, which are affected by the processing conditions. In section 1.2.2 experiments to characterize the mechanical properties of silica aerogels and xerogels are presented. They have shown that there is a power law relation between the elastic modulus and the density of the material as well as between the strength and the density. Those relations are characterized by exponents between 2.9 and 3.9 and between 2.3 and 2.8, respectively. Section 1.3 describes hybrid aerogels and xerogels, specially those fabricated by adding methyl groups $\left(\mathrm{CH}_{3}\right)$ into the silica structure $\left(\mathrm{CH}_{3}\right.$-added silica). It focuses on the experimental information available about how the structural and mechanical properties are affected by the addition of organic groups into the structure of the material. In that section, the molecules formed in $\mathrm{CH}_{3}$-added silica due to the combination of organic and inorganic precursors are identified since they will be necessary to build the computational model of the material. Contrasting findings about the effect of $\mathrm{CH}_{3}$ in the structure on the hardness and the strength of the samples determined by nanoidentation tests are presented. 


\subsection{Aerogel and Xerogel Materials}

Aerogels and xerogels are nanoporous materials with an open-cell structure formed by interconnected colloidal sphere-like particles or polymeric chains. Their porous structure contains pores ranging from 2 to $100 \mathrm{~nm}$ in size which leads them to have large surface areas and low densities. Aerogels, for instance, have surface areas ranging from 400 to $1000 \frac{\mathrm{m}^{2}}{\mathrm{~g}}$ and densities varying from 0.003 to $0.5 \frac{\mathrm{g}}{\mathrm{m}^{3}}$. Their large surface area, larger than that of any other known material, is due to their complex cross-linked internal structure, where the sphere-like colloidal particles or the polymeric chains are connected forming branches with a characteristic dimension of $10 \mathrm{~nm}$ [1]. Aerogels and xerogels became industrially important because they exhibit the hihgest electrical, thermal and sound insulation properties among all solid materials. Those properties added to their particular structure make them attractive for applications like dielectrics, electrode materials, membranes for desalination, precursors for advanced glasses and ceramics, substrates for catalysis, fuel cells, particle traps, high performance windows, sensors, lasers, and emitters of visible light [2]. Many of the outstanding properties of aerogels are more commonly found in a dense gas than in a solid material 3 .

Steven Kistler coined the term aerogel based on his work done in 1931 at the College of the Pacific (Stockton, CA). He proposed that a gel was composed of both liquid and solid phases which were independent of each other, and that if the liquid phase was separated in a non-destructive manner the remainder would be a highly porous solid spaning almost all the volume prior occupied by the wet gel [4]. To prove his hypothesis, Kistler prepared gels of silica $\left(\mathrm{SiO}_{2}\right)$ and tried to evacuate the liquid phase by evaporating the gel in air; but the occurring capillary tensions at the liquid/vapor interfaces caused collapsing of the structure, or a considerable shrinkage of it. He realized that, to avoid damaging the solid structure, the gel samples must be heated up and the pressure increased until the critical conditions of the liquid are reached. This allows draining the liquid without creating the liquid/vapor interface, because at the critical conditions there is no surface tension between liquid and gaseous states. His processing method became a key component of the industrial fabrication of aerogels [2]. Since slow evaporation of the liquid inside the gel at room temperature and atmospheric pressure is possible without breaking the silica structure but causing large shrinkage of it, the resulting porous materials are different from the aerogels because they have lower surface area, smaller pores and higher densities. Materials processed under those conditions are known as xerogels, and they have densities ranging from 0.8 to $1.3 \frac{\mathrm{g}}{\mathrm{m}^{3}}$ [5]. A different method to remove the liquid phase of the gel leads to the materials known as cryogels. In that case the liquid inside the wet gel is first frozen and then sublimed. The name is used because cryogenic conditions are needed to process the gel [3]. Understanding that the main difference between aerogels and xerogels is due only to the shrinkage, caused by the drying process, it can be considered that they have similar structural properties only differentiated by the density.

Kistler's first process to produce silica aerogels was based on the polycondensation of sodium 
silicate, also known as waterglass. The chemical reaction is as follows:

$$
\mathrm{Na}_{2} \mathrm{SiO}_{3}+2 \mathrm{HCl}+\mathrm{nH}_{2} \mathrm{O} \longrightarrow \mathrm{SiO}_{2} \bullet \mathrm{H}_{2} \mathrm{O}+2 \mathrm{NaCl}+(\mathrm{n}-\mathrm{x}+1) \mathrm{H}_{2} \mathrm{O}
$$

His early attempts to produce aerogels by evaporating the water at supercritical conditions failed because the water dissolved the silica, which was precipitated as the water was vented [2]. The critical conditions for water are reached at $21.8 \mathrm{MPa}(215 \mathrm{~atm})$ and $647 \mathrm{~K}$; in these conditions the silica is highly soluble in water [6]. Later, he designed a process where water was substituted by alcohol (ethanol) before the venting process. After the water was replaced the gels were referred as alcogels. Over the next years he characterized aerogels and xerogels prepared using materials like: alumina, tungsten oxide, ferric oxide, tin oxide, nickel tartrate, cellulose, cellulose nitrate, gelatin, agar, egg albumen, and rubber [2, 3]. One of his main conclusions was that, in theory, any multifunctional monomer that can be cross-linked to form a gel could be converted into an aerogel via supercritical extraction of the liquid [4].

No further work about aerogels was done until the 1970's, when the French government entrusted Stanislaus Teichner at Claude Bernard University with the development of a method for storing oxygen and rocket's fuel in porous materials. Teichner's group studied Kistler's method and realized that it was inefficient because it took long time to produce small quantities of aerogel. To solve that inconvenience, the group developed a process based on Sol-Gel chemistry. The new process replaced Kistler's sodium silicate $\left(\mathrm{Na}_{2} \mathrm{SiO}_{3}\right)$ with an alkoxysilane, namely tetramethoxysilane (TMOS or $\left.\mathrm{SiO}_{4}\left(\mathrm{CH}_{3}\right)_{4}\right)$. Hydrolyzing TMOS produces a gel constituted by a solid skeleton of silica combined with liquid methanol, which are the same constituents of Kistler's alcogels, in one step. This process eliminated the major drawbacks in Kistler's method: i) the water/alcohol exchange step, which was highly time consuming, because the porous $\mathrm{SiO}_{2}$ skeleton was filled by alcohol after the hydrolyzation, and ii) the presence of inorganic salts in the aerogel. Drying alcogels at the supercritical conditions of the alcohol resulted in high quality silica aerogels [2].

The hydrolysis of TMOS is a particular case of hydrolysis and polycondensation of organometallic precursors. The reaction proceeds as described in the following. Mixing the organometallic precursor, for instance $\mathrm{SiO}_{4} \mathrm{R}_{4}$, where $\mathrm{R}$ represents an alkyl group such as $\mathrm{CH}_{3}$ or $\mathrm{C}_{2} \mathrm{H}_{5}$, with water causes the formation of monosilicic acid $\left(\mathrm{Si}(\mathrm{OH})_{4}\right)$ and a form of alcohol $(\mathrm{R}(\mathrm{OH}))$. Simultaneously, the monosilicic acid polycondensates forming dimers $\left((\mathrm{OH})_{3}-\mathrm{Si}-\mathrm{O}-\mathrm{Si}-(\mathrm{OH})_{3}\right)$ and water. The excess of water accelerates the hydrolisis reaction.

$$
\begin{gathered}
\mathrm{SiO}_{4} \mathrm{R}_{4}+4 \mathrm{H}_{2} \mathrm{O} \underset{\&}{\longrightarrow} \mathrm{Si}(\mathrm{OH})_{4}+4 \mathrm{ROH} \\
2 \mathrm{Si}(\mathrm{OH})_{4} \longrightarrow(\mathrm{OH})_{3}-\mathrm{Si}-\mathrm{O}-\mathrm{Si}-(\mathrm{OH})_{3}+\mathrm{H}_{2} \mathrm{O}
\end{gathered}
$$

Further polycondensation produces additional $\equiv \mathrm{Si}-\mathrm{O}-\mathrm{Si} \equiv$ linkages until a coherent network exists [7]. Those two reactions are strongly dependent on the $\mathrm{pH}$ and are catalyzed by bases or acids, 
changing the final characteristics of the aerogel $[8]$.

For the case of TMOS the reaction is as follows

$$
\mathrm{SiO}_{4}\left(\mathrm{CH}_{3}\right)_{4}+\mathrm{H}_{2} \mathrm{O} \longrightarrow \mathrm{SiO}_{2}+4 \mathrm{CH}_{3} \mathrm{OH}
$$

The main drawback of the application of this technique is that the alcohol produced during the hydrolysis, $\mathrm{CH}_{3} \mathrm{OH}$ (methanol), reaches critical conditions at $516 \mathrm{~K}$ and $8.1 \mathrm{MPa}(80 \mathrm{~atm})$ and it is highly explosive in that state. The risk involved in the process was confirmed in 1984 when a plant located in Lund, Switzerland, exploded. Additional risks arise because TMOS is a toxic reagent [6].

In 1983 the Microstructured Materials Group at Lawrence Berkeley Lab (LBL, Berkeley, CA), under direction of Arlon Hunt, found that the TMOS could be replaced with tetraethylorthosilicate (TEOS, $\left.\mathrm{Si}\left(\mathrm{C}_{2} \mathrm{H}_{5}\right)_{4}\right)$, a less toxic reagent. LBL's group also discovered that the alcohol within the gel, ethanol $\mathrm{C}_{2} \mathrm{H}_{5}(\mathrm{OH})$ in this case, could be replaced by liquid $\mathrm{CO}_{2}$ before drying. That discovery represented a big advance in safety because the critical point of $\mathrm{CO}_{2}$ is reached at $304 \mathrm{~K}$ and 74 $\mathrm{MPa}(73 \mathrm{~atm})$, less severe conditions than those for methanol or ethanol. In addition, $\mathrm{CO}_{2}$ is non explosive. The process to produce an aerogel can be schematized as in the Fig. 1.1.

In spite of the advances in the understanding of the properties of the aerogel and xerogels materials they have not found a lot of industrial applications specially due to the production cost. However, it is clear that in several industrial cases, such as thermal insulation, glass precursors, and support for catalysts, aerogels and xerogels will give a better performance than more traditional materials. Recent efforts are oriented to overcome the processing limitations and to make this type of materials economically competitive [6].

Developments include the implementation of techniques to facilitate the drying of the samples at atmospheric pressure, without causing excessive shrinkage of the structure. One interesting new technique consists of adding reagents to the solution which will modify either the properties of the solid structure, making it able to support the capillary forces, or the liquid phase, reducing the capillary tensions developed. The main goal is to reduce the costs caused by processing the material at the supercritical conditions of the liquid phase. Additionally, the use of cheap precursors has being considered, the sodium silicate used by Kistler to produce waterglass is cheaper than the organometallic precursos (like TMOS or TEOS) but the process must be modified to make it efficient [6].

Another drawback of the traditional process is that heating up the wet gel to reach the critical temperature of the liquid takes a lot of time. The differences in the expansion coefficients of liquid and solid phases. makes necessary the use of slow heating rates to avoid cracking the solid phase. New processes have been designed in which the processing time has been reduced about 30 times 


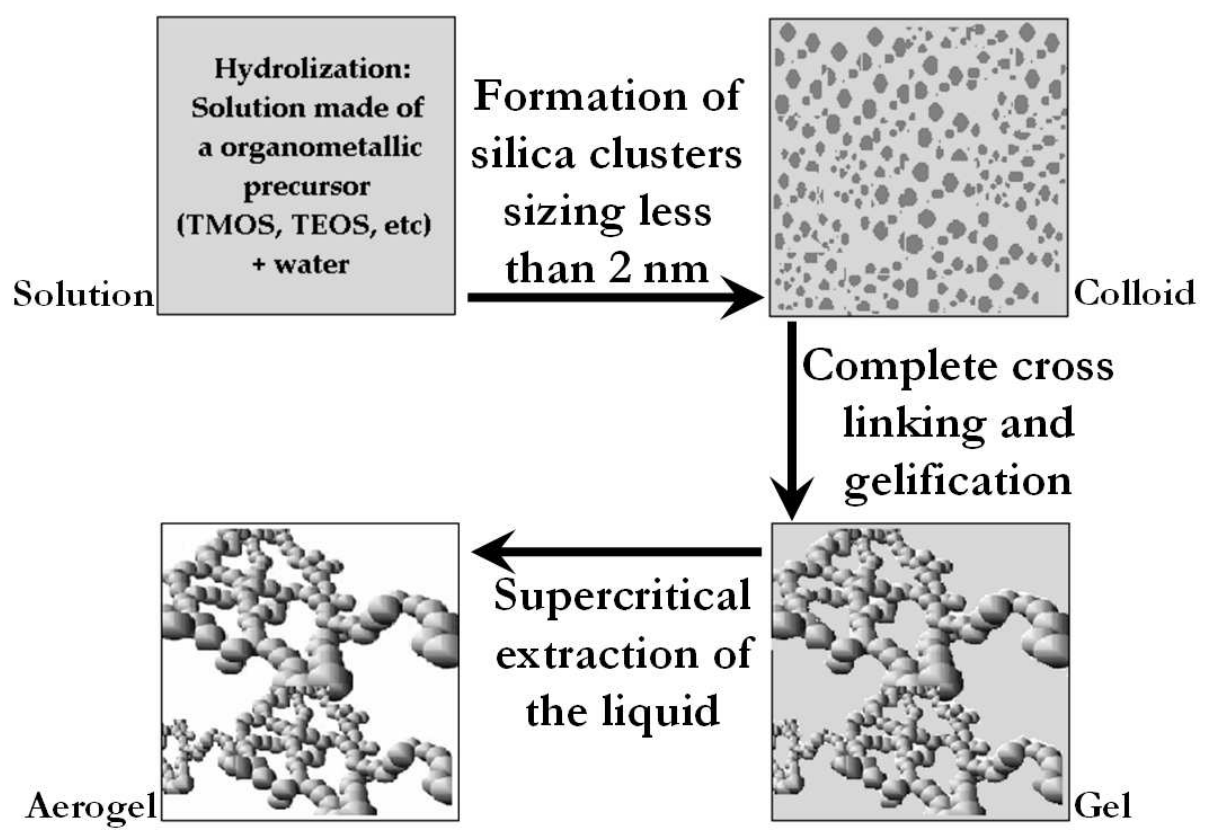

Figure 1.1: Schematic of the process to make an aerogel

by combining the stages of gelation and drying in one process [6]. An additional set of barriers for the generalized use of aerogels and xerogels comes from their poor mechanical properties. Their low strength and Young's modulus make them difficult to manipulate. Even though some aerogels can support 1000 times their own weight, most of people crush them the first time they handle them 2 .

\subsection{Silica Aerogels and Xerogels}

\subsubsection{Structure of Silica Aerogels and Xerogels}

Gesser [3] reviewed some of the advances on the understanding of the internal structure of aerogels. He summarized the initial developments on measuring the pore size distribution and the description of the internal structure of the material. Measurements of pore size distribution by mercury porosimetry and adsorption/desorption of nitrogen have shown that this type of materials have pores with sizes varying in the nanometer range, from 1 to $100 \mathrm{~nm}$ (micro to mesoporous range), with an average pore size of about $40 \mathrm{~nm}[9]$.

High resolution electron microscopy (HREM), electron spectroscopy for chemical analysis (ESCA) and Auger spectroscopy have allowed defining the structure of these materials as composed by a complex network of interconnected sphere-like particles of pure stoichiometric silica sizing about 10 nm. Tewari et al. (as cited in [3]) used Rayleigh scattering and transmission electron microscopy (TEM) to study the internal structure of the aerogel obtaining sizes of internal components of about $8 \mathrm{~nm}$ and $5 \mathrm{~nm}$ with each technique respectively. Small angle X-ray scattering (SAXS) data 
presented by Schuck (as cited in [3]) confirmed that the most frequent particle diameter in the sphere-like chains was in range of 5-6 $\mathrm{nm}$.

The understanding of the gelation process along with the results of microscopic examinations, scattering experiments and different analytical models have led to the conclusion that silica aerogels and xerogels, in most of the cases, can be treated as open cell foams with a fractal structure. Woignier et al. [10] compared the elastic properties of a model for aerogels based on percolation theory with experimental results. They found that the fractal structure of the aerogels is far more complex than the one that can be deduced from a straight forward application of the percolation theory. Nevertheless they showed that a qualitative description of the material could be made based on a percolation model.

Fricke et al. [9] outlined a study of the structure of silica aerogel based on the relation between the density and the length scale of the sample, a technique commonly used to analyze porous materials. They remarked that the aerogels have a fractal structure which was clearly identified in samples prepared under acid-catalyzed conditions; a fractal dimension around 2.4 was estimated from scattering experiments. Their experiment with base-catalyzed aerogels did not reveal strong fractality.

The fractal dimension, as referred by Fricke et al. [9, is explained using the Fig. 1.2, When the density of a large piece of a porous material is measured the value obtained corresponds to the macroscopic or apparent density of the sample. If the scale of the sample (its size) is progressively reduced, the effect of the porosity starts having an effect, and the measured density is larger than the apparent density. When the selected scale is small enough one reaches the skeletal density of the porous material, which is larger than the apparent density of the sample. The form in which the density and the scale relate in the region between the apparent density and the skeletal density determines the fractality of the material. If there is a linear relation between the logarithm of density and the logarithm of the linear length scale the porous sample has a fractal structure, otherwise it does not [9].

Further analysis of the fractality of silica aerogels were performed by Woignier et al. [11. They prepared samples under acid, neutral and basic conditions. After analyzing the results of small angle neutron scattering (SANS) they concluded that all the samples had a fractal structure, which was characterized by a fractal dimension that changed with the $\mathrm{pH}$ of the solution, and that was independent of the concentration of the silica precursor. They found that the fractal dimension was around 2.3 for aerogel prepared under neutral conditions, around 2.4 for those prepared in acidic solutions, and around 1.8 for samples under basic conditions. Their results could be explained by the fractal growth of the clusters prior to gelation. During the gelation of a solution only cross-linking of previously formed clusters occur without changing the internal structure of the 


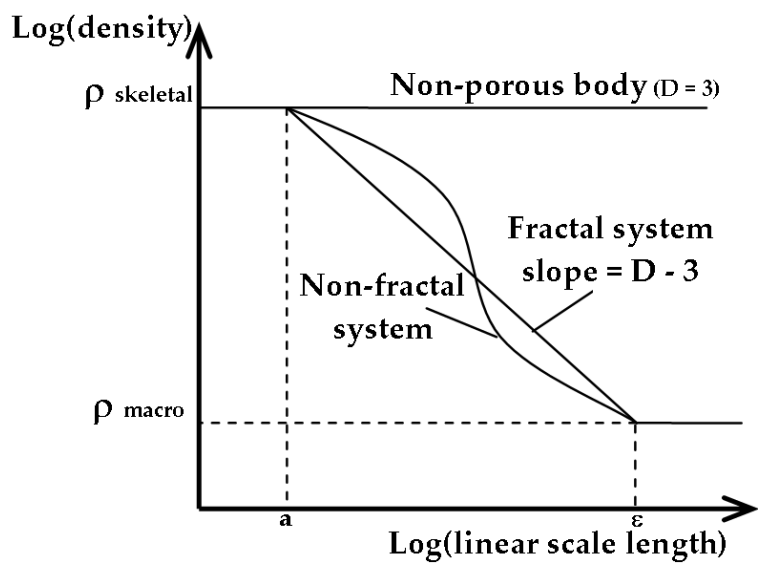

Figure 1.2: Dependence of the density of a porous system on the scale length. $a$, size of the smallest building block. $\epsilon$, correlation length. D, fractal dimension of the object

clusters. However, the fractal characteristics of the aerogels structure can be influenced by other factors, such as, aging time of the gel prior to extraction of the liquid and the amount of shrinkage experienced during the drying process [11].

In a more recent study, Woignier et al. [8] used SAXS to characterize the structure of five different kinds of silica aerogel and xerogel samples prepared by TMOS hydrolization and supercritical drying. Three groups of samples were catalyzed under acid, base and neutral conditions, one group was isostatically compressed using a mercury porosimetry device (called partially compressed samples), and the last group was partially densified by sintering (called partially densified samples). The total set of samples had porosities varying from $0 \%$ to $98 \%$, a range of porosities covering both aerogels and xerogels. Scattering experiments confirmed the values of the fractal dimension found previously [11], even for the samples that were partially compressed and partially densified. The study showed that for aerogel samples catalyzed under neutral, acidic, and basic conditions developed fractal features characterized by fractal dimensions of $2.4 \pm 0.1,2.2 \pm 0.1$, and $1.8 \pm 0.1$, respectively. The density mainly affected the extension of the fractal range, being greater for samples with lower density. For partially densified samples they found that the fractal dimension increases with the density, and for the partially compressed samples, it remains essentially constant.

The use of techniques such as nuclear magnetic resonance (NMR), Brunauer, Emmett, and Teller (BET) adsorption/desorption analysis, SAXS or mechanical testing has led to classify the aerogels as: i) Polymeric aerogels, for those having larger surface area, higher stiffness and a fibrous microstructure characterized by a fractal dimension of about 2.4; in silica aerogels those properties correspond to acid-catalyzed samples, and ii) Colloidal aerogels, which have smaller surface areas, lower stiffness and a microstructure composed of interconnected spheres with limited fractal characteristics, in silica aerogels these features correspond to base-catalyzed samples. In spite of the knowledge about the different types of structures of the aerogels and xerogels, there is no a well 
defined line that separates the processing conditions leading to one type of structure or the other; it is more a continuous transition allowing colloidal and polymeric regions to coexist [1].

Ruben et al. [1] produced samples of resorcinol-formaldehyde (RF) aerogel with different proportions of resorcinol to catalyst $(\mathrm{R} / \mathrm{C})$ and base-catalyzed aerogels. After characterizing the samples using BET adsorption/desorption and mechanical tests, they concluded that RF aerogels with low R/C should have a microstructure similar to that of acid-catalyzed silica aerogels, while the other samples of RF aerogel (high R/C) should be similar to base-catalyzed silica aerogels.

Ruben et al. [1] replicated the structure of the aerogels by growing a thin layer of platinumcarbon ( $\sim 1 \mathrm{~nm}$ thick) on the surface of the samples, cutting them under cryogenic conditions, and imaging their microstructure using HRTEM. The replication technique allowed them to resolve the structural features of the material at the molecular level, identifying structures of about $1 \mathrm{~nm}$ in size. For the colloidal aerogels (base-catalyzed silica and high R/C RF samples) they found that the structural branches were formed by many spherical particles connected like a chain of beads, whose sizes varied depending on the $\mathrm{R} / \mathrm{C}$ value. For the silica aerogels the bead size ranged from 8.6 to $13.2 \mathrm{~nm}$ having an average value of $11.3 \pm 0.1 \mathrm{~nm}$. For the polymeric aerogels (RF aerogels with properties similar to acid-catalyzed silica aerogel) they found that the branches were made of highly convoluted chains forming filaments of $2-6 \mathrm{~nm}$ in diameter, a feature that makes the structure even more open than that of base-catalyzed samples. This could account for both the larger surface area and the higher stiffness of polymeric aerogels. Their conclusions about the polymeric RF aerogels could be extended to acid-catalyzed silica aerogels.

\subsubsection{Mechanical Properties of Aerogels and Xerogels}

The internal structure of aerogels and xerogels is responsible for their outstanding properties, such as large surface area and low thermal conductivity; but it also makes these materials relatively weak from the point of view of the mechanical response. The tensile strength and the elastic modulus of aerogels and xerogels can be up to four orders of magnitude smaller than those of dense silica glass.

Woignier et al. [10] measured the mechanical properties of silica aerogel samples having den-

sities from 100 to $400 \frac{\mathrm{kg}}{\mathrm{m}^{3}}$, equivalent to porosities between $95 \%$ and $78 \%$ calculated considering a skeletal density of $1850 \frac{\mathrm{kg}}{\mathrm{m}^{3}}$ obtained by Helium pycnometry at room temperature. They used monolithic samples of $150 \times 8.5 \times 8.5 \mathrm{~mm}^{3}$ loaded in a three point bending configuration to determine a relation between the density and the Young's modulus of the material. They found that the Young's modulus is one or two orders of magnitude smaller than the corresponding for a fully dense material. They also determined that for silica aerogels and silica xerogels there is a power law dependency between the Young's modulus and the density, $E \propto \rho^{\alpha}$, with $\alpha$ varying from 3.0 to $4.0[8,10]$ 
Silica and Hybrid Aerogels and Xerogels Materials

\begin{tabular}{llll}
\hline Reference & Exponent & Density $\left[\frac{\mathbf{g}}{\mathbf{c m}^{3}}\right]$ & Type of sample \\
\hline Woignier et al. $[\mathbf{8}]$ & $3.7 \pm 0.2$ & $0.055-0.5$ (approx. values) & $\mathrm{pH}$ neutral, acidic and basic \\
\hline Woignier et al. $[\mathbf{8}]$ & 3.2 & $0.42-2.2$ (approx. values) & Partially densified samples \\
\hline Woignier et al. [10] & 3.8 & $0.1-0.4$ (approx. values) & $\mathrm{pH}$ neutral \\
\hline Groß et al. [12] & $3.49 \pm 0.07$ & $0.14-2.7$ & \\
\hline Groß et al. [12] & $2.97 \pm 0.05$ & $0.08-1.2$ & Sintered aerogels \\
\hline
\end{tabular}

Table 1.1: Exponent for the power law relation between elastic modulus and density of porous silica.

\begin{tabular}{llll}
\hline Reference & Exponent & Density $\left[\frac{\mathbf{g}}{\mathbf{c m}^{3}}\right]$ & Type of sample \\
\hline Woignier et al. $[8]$ & $2.6 \pm 0.2$ & $0.055-0.5$ (approx. values) & pH neutral, acidic and basic \\
\hline Woignier et al. $[8]$ & 2.3 & $0.42-2.2$ (approx. values) & Partially densified samples \\
\hline
\end{tabular}

Table 1.2: Exponent for the power law relation between strength and density of porous silica.

Woignier and Phalippou [5] extended the previous study by including the diametral compression tests (brazilian test), and developing a model to predict the mechanical properties of the silica aerogel based on some textural properties of the material such as pore volume, mean particle radius, and mean neck radius. Their model of the aerogel structure consisted of spheres of silica connected by small necks forming a frame of cubic cells. The radius of the silica spheres and the volume of the pores where calculated based on the pore size distribution obtained from the BET method. Their model needed to adjust the ratio between the radius of the necks and the silica spheres to match the experimental results; nevertheless, it probed to be an interesting and simple approach to predict the strength of the aerogels. More important than the model they proposed are the experimental results they presented, which show that, similarly to the Young's modulus, the strength of the silica aerogels relates to the density following a power law. 10 years later, Woignier et al. [8] published new results of similar tests, again confirming the power law relation between Young's modulus and density as well as between strength and density.

Groß and Fricke [12] used the ultrasonic pulse-echo method to study the relation between elastic modulus and density. A power law relation between elastic modulus and density was also found using this technique. Ruben et al. also reported a power law relation between Young's modulus and density, with an exponent varying from 2.0 to 4.0; for their base-catalyzed silica aerogels the exponent was $2.9 \pm 0.2$.

The exponents of the power law relations, as well as the range of densities used for the experiments are summarized in Tables 1.1 and 1.2 .

\subsection{Hybrid Aerogels and Hybrid Xerogels}

Since aerogels and xerogels can be produced from both organic and inorganic precursors, the term hybrid aerogel or hybrid xerogels will apply to aerogels and xerogels composed of organic and inor- 
ganic components. Some common hybrid aerogels and xerogels are produced combining silica and alkyl groups $\left(\mathrm{C}_{m} \mathrm{H}_{n}\right)$, for instance methyl groups $\left(\mathrm{CH}_{3}\right)$, ethyl groups $\left(\mathrm{C}_{2} \mathrm{H}_{5}\right)$, or propyl groups $\left(\mathrm{C}_{3} \mathrm{H}_{7}\right)$. Those materials are also known as organo-modified silicas.

Hybrid aerogels and xerogels are fabricated to obtain products with enhanced properties compared to materials made of simpler compounds. For instance, organo-modified silicas are studied because they can provide hydrophobicity to the products, functionalizable surfaces for specific applications, or materials with improved mechanical properties [13, 14]. There are four main mechanisms for incorporating organic components in an inorganic network, namely, i) interpenetrating organic-inorganic networks, where two non-bonding networks are grown during the sol-gel process, ii) embedded molecules, where non-bonding molecules are trapped inside the aerogel/xerogel structure, iii) dual networks, and iv) functionalized structures. For the last two cases the organic molecules are bonded to the inorganic structure through $\mathrm{Si}-\mathrm{C}$ bonds. The non-bonding structures are difficult to achieve because the organic components tend to be washed out during the drying process, in particular if it is performed at supercritical conditions. Therefore, hybrid structures based on the covalent bonding of $\mathrm{Si}$ and $\mathrm{C}$ atoms are the most common [13].

\subsubsection{Structure of $\mathrm{CH}_{3}$-Added Silica Aerogels and Xerogels}

The usage of methyl groups in the silica structure has been traditionally done to change the natural hydrophilic character of silica aerogels and xerogels as gelled to a hydrophobic character [15, 16, 17, 18].

Using hydrolyzed mixtures of TMOS $\left(\mathrm{Si}\left(\mathrm{OCH}_{3}\right)_{4}\right)$ and methyltrimethoxysilane (MTMS or $\left.\mathrm{CH}_{3} \mathrm{Si}\left(\mathrm{OCH}_{3}\right)_{3}\right)$ hydrophilic aerogels and xerogels are produced because the hydrophilic -OH groups formed on the surface of silica after hydrolyzation of TMOS are replaced by hydrophobic $-\mathrm{CH}_{3}$ produced during the hydrolyzation of trimethoxymethylsilane (TRIMOS). The change in the nature of the surface of silica is due to the fact that TMOS hydrolyzes faster than TRIMOS, since the hydrolyzation of TMOS creates the $\mathrm{SiO}_{2}$ frame of the aerogel, while the hydrolyzation of TRIMOS produces the hydrophobic groups formed on the surface of the material [15, 16, 17]. The characterization of of the samples used by Schwertfeger et al. [15, 16, 17] showed that methyl groups can appear in the final structure of the aerogel or xerogel bonded to Si or O atoms effectively inhibiting the formation of O-Si-O bonds. Experiments performed by Tillison et al. [19] reported that the addition of organic groups changes the structural features of the aerogels increasing the surface area of the samples.

Kanamori et al. [14] prepared aerogels and xerogels using methyltrimethoxysilane (MTMS or $\left.\mathrm{CH}_{3} \mathrm{Si}\left(\mathrm{OCH}_{3}\right)_{3}\right)$ as the only silica precursor. The expected structure after the hydrolysis and gelation of MTMS is composed of $\mathrm{CH}_{3} \mathrm{SiO}_{1.5}$ molecules, called methylsilesquioxane (MSQ). However due to the strong hydrophobicity of the methyl $\left(-\mathrm{CH}_{3}\right)$ groups, phase separation is highly likely to 
(a)

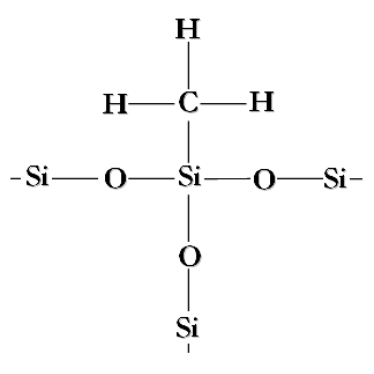

(b)

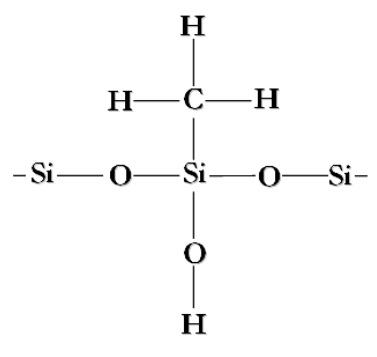

(c)

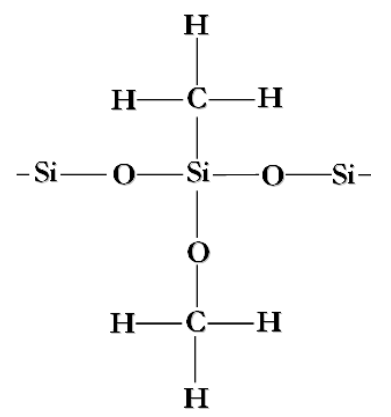

Figure 1.3: Molecules formed after the hydrolysis and polymerization of MTMS [14. (a) Fully condensed (MSQ). (b) Partially condensed with silanol group (OH). (c) Partially condensed with additional methyl group $\left(\mathrm{CH}_{3}\right)$

occur in the solution leading to the formation of aggregates or stable solutions instead of gels. In the preparation procedures described in [14 the use of surfactants to prevent the phase separation is explained. Through the use of solid-state NMR spectroscopy, Si cross polarity magic angle spinning NMR (Si CP/MAS NMR), Kanamori et al. showed that the hydrolysis and condensation of MTMS leads to the formation of three types of molecules: i) fully condensed molecules of the form $\mathrm{CH}_{3} \mathrm{Si}(\mathrm{OSi})_{3}$, which are equivalent to MSQ molecules, ii) partially condensed molecules of the form $\mathrm{CH}_{3} \mathrm{Si}(\mathrm{OSi})_{2}(\mathrm{OH})$, and iii) partially condensed molecules of the form $\mathrm{CH}_{3} \mathrm{Si}(\mathrm{OSi})_{2} \mathrm{CH}_{3}$. Schematic representations of those molecules are presented in the Fig. 1.3. Kanamori et al. [14] also found that the amount of partially condensed molecules varied for the two different types of surfactants used in their experiments, $n$-hexadecyltrimethylammonium bromide (CTAB) and poly(ethylene oxide)-block-poly(propylene oxide)-block-poly(ethylene oxide) $\left(\mathrm{EO}_{106} \mathrm{PO}_{70} \mathrm{EO}_{106}\right.$, F127). For samples prepared under the same conditions but with different surfactant, the ratio of partially to fully condensed molecules was 0.22 (2:9) for CTAB and 0.29 (5:17) for F127. In [14] there is no reference about either the ratio between the type of partially condensed molecules or the relation between the amount of partially condensed particles and the density of the samples.

Hybrid aerogels were prepared by Martin et al. [18] using combinations of a traditional silica precursor, TMOS, and a silica precursor that incorporates organic components into the final structure of the aerogel (TRIMOS). The aerogels produced were formed by four types of molecules, which were identified using Si-CP/MAS-NMR and C-CP/MAS-NMR techniques. Schematic representations of those molecules are shown in Fig. 1.4. Molecules of type $\mathrm{Q}_{4}$ and $\mathrm{Q}_{3}$ correspond to the hydrolysis of TMOS in traditional silica aerogels. Molecules of type $T_{3}$ and $T_{2}$ are formed due to the presence of TRIMOS in the solution, and correspond to MSQ and partially condensed molecules as identified by Kanamori et al. [14. However, molecules of the form $\mathrm{CH}_{3} \mathrm{Si}(\mathrm{OSi})_{2} \mathrm{CH}_{3}$, characterized by the presence of two methyl groups, were not found in the samples. The amount of each type of molecule for different ratios of the mixture TMOS:TRIMOS is presented in Table 1.3 , 


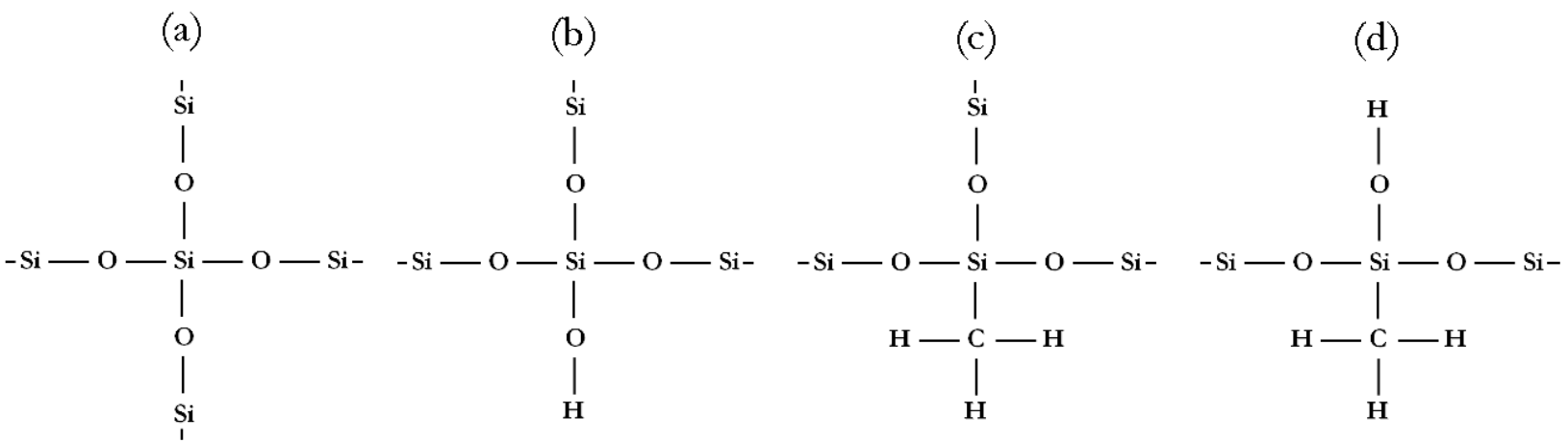

Figure 1.4: Molecules formed after the hydrolysis and polymerization of TMOS:TRIMOS mixtures [18. (a) $\mathrm{Q}_{4}$ type, (b) $\mathrm{Q}_{3}$ type, (c) $\mathrm{T}_{3}$ type, and $\mathrm{T}_{2}$ type.

\begin{tabular}{ccccc}
\hline TMOS:TRIMOS & $\mathbf{Q}_{4}[\boldsymbol{\%}]$ & $\mathbf{Q}_{3}[\boldsymbol{\%}]$ & $\mathbf{T}_{3}[\boldsymbol{\%}]$ & $\mathbf{T}_{4}[\boldsymbol{\%}]$ \\
\hline $100: 0$ & 62.23 & 37.77 & - & - \\
\hline $90: 10$ & 52.68 & 29.78 & 11.33 & 6.21 \\
\hline $60: 40$ & 42.25 & 12.67 & 31.89 & 13.19 \\
\hline $50: 50$ & 38.22 & 7.77 & 41.78 & 12.23 \\
\hline $45: 55$ & 34.27 & 7.63 & 45.64 & 12.46 \\
\hline $40: 60$ & 28.26 & 4.75 & 52.79 & 14.19 \\
\hline
\end{tabular}

Table 1.3: Percentage of the different type of molecules formed after the condensation of TMOS:TRIMOS mixtures [18.

\subsubsection{Mechanical Properties of $\mathrm{CH}_{3}$-Added Silica Aerogels and Xerogels}

Aerogels and xerogels prepared by Kanamori et al. 14] from MTMS showed improved mechanical properties under compression. Their samples could be uniaxially compressed up to $80 \%$. After the load is released, the samples spring-back to almost the original length without major damage. These levels of deformation can not be achieved with samples made of silica only. Three factors are considered responsible for the improved compressibility of the MSQ aerogels: i) lower cross-linking of the structure because silicon atoms are only bonded to three oxygen atoms (siloxane groups, -SiO-), while in pure silica structures silicon atoms are bonded to four oxygen siloxane groups,

\begin{tabular}{cccc}
\hline TMOS:TRIMOS & $\left.\begin{array}{c}\text { Density } \\
{[ \pm \mathbf{0 . 0 1}}\end{array} \frac{\mathbf{g}}{\mathbf{\mathbf { g m } ^ { 3 }}}\right]$ & $\begin{array}{c}\mathbf{E} \\
{[\mathbf{M P a}]}\end{array}$ & $\begin{array}{c}\text { Hardness } \\
{[\mathbf{M P a}]}\end{array}$ \\
\hline $100: 0$ & - & 31.3 & 5.1 \\
\hline $90: 10$ & 0.16 & 22.6 & 4.9 \\
\hline $85: 15$ & 0.13 & 23.8 & 6.0 \\
\hline $75: 25$ & 0.12 & 16.0 & 5.0 \\
\hline $60: 40$ & 0.11 & 16.0 & 6.4 \\
\hline $45: 55$ & 0.13 & 14.9 & 5.8 \\
\hline $40: 60$ & 0.13 & 13.0 & 5.0
\end{tabular}

Table 1.4: Elastic modulus and Hardness of samples prepared using different TMOS:TRIMOS mixtures [18. 
making the material stiffer, ii) the lower density silanol groups $(-\mathrm{OH})$ reduce the permanent shrinkage of the structure increasing the spring back of the system when the load is removed, and iii) methyl groups $\left(-\mathrm{CH}_{3}\right)$, which repel from each other, are homogeneously distributed in the structure favoring the spring back of the system. The compliance obtained in this type of aerogels makes ambient pressure drying possible without significant damage of the samples [14].

Martin et al. [18] also studied the mechanical properties of the organo-modified aerogels by performing nanoindentation tests to samples prepared with different TMOS:TRIMOS mixtures. They found that as the amount of organic molecules in the structure increases, the elastic modulus of the material is reduced while the hardness remains constant, see Table 1.4. The hardness of the material is related to its strength. Therefore, for materials having similar hardness, the compliance, which is related to the inverse of the Young's modulus, determines the toughness. In the case of the aerogel samples studied in [18], increasing the amount of organic molecules in the structure increases the compliance of the samples making them tougher.

De la Rosa-Fox et al. 20] also studied the mechanical characteristics of hybrid aerogels by using nanoindentation and creep tests. Their samples were prepared using mixtures of tetraethylorthosilicate (TEOS) and polydimethylsiloxane (PDMS or $\left.\left(\mathrm{CH}_{3}\right)_{3} \mathrm{SiO}\left(\left(\mathrm{CH}_{3}\right)_{2} \mathrm{SiO}\right)_{n} \mathrm{Si}\left(\mathrm{CH}_{3}\right)_{3}\right)$. They found that addition of organic components changes the behavior of the material from a brittle solid to a rubber elastomer; a result that was also found by Piñero et al. [21] who studied the compressive properties of aerogels produced using the same precursors. From experimental results presented in [20, it is clear that the Young's modulus of the hybrid aerogels is reduced when the amount of organic material added is increased. However, it was also found that the hardness of the samples is reduced as well; a result that contradicts the findings of Martin et al. [18. The changes of the mechanical properties of hybrid aerogels are due to the reorganization of the internal structure of the material when the amount of organic material is increased. If the structure has a small organic content, the mechanical response is dominated by the brittle inorganic structure, but when the amount of organic material surpasses a certain level an organic matrix is formed and the material becomes rubbery [20, 21]. 


\section{Chapter 2}

\section{Molecular Dynamics Simulation Technique}

The fundamental concepts of Molecular Dynamics simulations are presented in this chapter. A brief overview of the technique is presented first, including essential aspects for a simulation such as statistical ensembles, interatomic potentials, initial and boundary conditions, integration of the equations of motion, and the theory of constraint dynamics, which is useful to model rigid molecules in atomic systems. This chapter also illustrates some of the methods used to estimate important macroscopic properties of atomic systems, namely temperature, kinetic energy, pressure, and mechanical stress. The final section of the chapter deals with the study of the fractal dimension of aerogels and xerogels, which is a quantity used to characterize their structure. 


\subsection{Molecular Dynamics Simulation Technique}

One of the first ideas of performing molecular dynamics simulations could be attributed to Pierre Simon Laplace, who around 1814 envisioned a hypothetical instance that could look at anytime into the past or future of the universe by only knowing the positions and momenta of all the particles at a particular instant of time. Laplace's vision was based on the basic ideas of classical mechanics, namely, i) all the processes are considered to be deterministic, and ii) the fundamental equations describing the phenomena occurring in the universe are time reversible. For a complete knowledge of the evolution of the universe the Laplace's daemon, as this hypothetical entity was called, needed to analyze the universe as an isolated system of particles whose behavior is fully determined by the solution of the classical equations of motion [22].

$$
\dot{p}_{i}=-\frac{\partial H}{\partial q_{i}} \quad \dot{q}_{i}=\frac{\partial H}{\partial p_{i}}
$$

Where $H$ is the Hamiltonian of the system, and $p_{i}$ and $q_{i}$ are the generalized momenta and coordinates of the $i^{\text {th }}$ particle. Nowadays, this vision has to be complemented with the theory of chaos and by quantum mechanics; nevertheless, the basic idea of the Laplace's daemon is present in modern particle simulations, a field that has become increasingly useful to understand the behavior of systems at scales where experimentation is either cost-restricted or impossible. Once a simulation model has been built it becomes a powerful tool to investigate the properties of a particular system. It also acts as a bridge between the microscopic/nanoscopic length and time scales and the macroscopic world of the laboratory, even though, a direct extrapolation of the results from particle simulations to macroscopic properties is not always straight forward [22].

Computer simulations allow applying almost any imaginable condition to analyze the response of a system. For instance, extremely high pressures and/or temperatures can be imposed to a computational system without using expensive devices and without safety concerns. In this way, computer simulations are very useful to verify theoretical developments at low cost and complement experimental research.

In the case of molecular dynamics, a program to simulate a system must be implemented describing:

i. A statistical ensemble where thermodynamic quantities, like pressure, temperature, energy and/or number of particles can be controlled. Statistical ensembles work as a mean to represent the actual characteristics of the system under study, for instance, mass transfer phenomena would be modeled by allowing the number of particles in the system to change during the simulation.

ii. An interatomic potential to represent the interactions between the system's constituents, namely atoms, molecules, free surfaces, etc. Generally, it is assumed that the particles interact 
at least pair-wise, which is exact in some cases (particles with fixed partial charges [22]).

iii. A set of initial and boundary conditions.

iv. An integration procedure to solve the equations of motion and to propagate the particle positions and velocities from time $t$ to $(t+\Delta t)$. The integration procedure is a finite difference scheme in which the time step $\Delta t$ must be carefully selected to guarantee stability of the program, i.e. there should be no drift in the system energy [22]. The fact that, at the atomic level, different phenomena happen at different time scales makes it difficult to select an adequate time step. However, it is usually selected as a fraction of the period of the fastest phenomenon being studied. The use of very small time steps seems to be appropriate but it leads to highly time consuming simulations and the need of sophisticated computer facilities to speed up the calculations.

v. A methodology to determine the physical properties of the system, e.g. temperature, pressure or energy, and other quantities of interest, e.g. stress and strain, based on the knowledge of the atomic positions and velocities.

As in any other type of simulation technique, the results of MD simulations need to be compared to experimental and/or theoretical information, and the interaction model should be refined accordingly. The interaction models are developed based on measurements of neutron diffraction, nuclear magnetic resonance and/or in theoretical formulations like ab-initio quantum mechanics [22].

\subsubsection{Statistical Ensembles}

During a molecular dynamics simulation all the information available about the system consists of atomic positions and velocities (or momenta), in some cases rotational speeds or momenta are also available. Therefore, means to relate that information to measurable, macroscopic variables are needed. To do this, statistical ensembles are used.

The time evolution of a system is represented by an orbit or trajectory in the so called Gamma phase space, $\Gamma$; a hyperspace composed by $6 \mathrm{~N}$ axes representing the $3 \mathrm{~N}$ coordinates and the $3 \mathrm{~N}$ velocities (or momenta) of the particles in the system. The physical properties for an atomic system, namely generalized positions, generalized momenta, temperature, pressure, energy, etc, are estimated based on averages of the trajectories of the system in the $\Gamma$ phase space. A statistical ensemble is defined as the group of trajectories in the $\Gamma$ phase space having the same average physical properties [23].

Common statistical ensembles are: i) the Microcanonical ensemble, where the number of particles, the volume, and the energy, E, are constants; it is also known as NVE ensemble, ii) the Canonical ensemble, where the number of particles, $\mathrm{N}$, the volume, $\mathrm{V}$, and the temperature, $\mathrm{T}$, 
are constant; it is also known as NVT ensemble, iii) The Isothermal-Isobaric ensemble, having constant temperature and pressure and, iv) the Grand Canonical ensemble, having a constant chemical potential, $\mu$, volume, and temperature. Among those statistical ensembles two are used for this research, the microcanonical and canonical. The former is used during the generation of the samples when the system needs to be equilibrated. The latter is used for the processes related with the generation of samples and during the evaluation of the mechanical properties through the simulation of tension tests.

\subsubsection{Interatomic Potential}

The interatomic potential is a critical component of the molecular dynamics simulation program. Its importance is easily explained when the dynamics of the system is expressed in terms of the Hamiltonian as

$$
\mathscr{H}(p, q)=\mathscr{K}(p)+\mathscr{V}(q)
$$

where $q, p, \mathscr{K}$, and $\mathscr{V}$ are the generalized coordinate, generalized momenta, the kinetic energy function, and potential energy function, respectively. The Hamiltonian takes the form

$$
\begin{gathered}
\mathscr{H}=\frac{1}{2} p^{T}[M]^{-1} p+\frac{1}{2} w^{T}[\theta]^{-1} w+\sum_{i=1}^{N-1} \sum_{j>i}^{N} V_{2}\left(r_{i j}\right)+\sum_{i=1}^{N-2} \sum_{j>i}^{N-1} \sum_{k>j}^{N} V_{3}\left(r_{i j}, r_{i k}, r_{j k}\right)+\ldots \\
\cdots+\sum_{i=1}^{N-3} \sum_{j>i}^{N-2} \sum_{k>j}^{N-1} \sum_{l>k}^{N} V_{4}\left(r_{i j}, r_{i k}, r_{i l}, r_{j k}, r_{j l}, r_{k l}\right)+\ldots
\end{gathered}
$$

The first and second terms in Eq. 2.3 represent the kinetic energy for translations and rotations of the particles. The third and additional terms represent the potential energy due to the interaction between two particles, three particles, four particles, and so on.

From this formulation it can be seen that the potential selected represents a large part of the Hamiltonian, and it can include as many interactions as deemed necessary to represent the desired system. Usually only pair-wise interactions are considered neglecting triplets and higher order interactions; However, potentials including triplets interactions are also found in the literature.

The importance of the interatomic potential is also reflected when the dynamics of the system is written in the form of Newton's second law (Eq. 2.4), because the interatomic forces are calculated directly from the potential formulation as indicated in Eq. 2.5

$$
m_{i} \ddot{\overrightarrow{r_{i}}}=\overrightarrow{f_{i}}
$$




$$
\vec{f}_{i}=-\frac{\partial V}{\partial \vec{r}_{i}}
$$

The contributions to the interatomic potential can be separated in two groups, one containing the interactions between atoms bonding to each other, and one containing the interactions between atoms that do not form bonds between each other. This division could be useful for modeling complex systems like long hydrocarbon chains or complex proteins. A model can be constructed by creating pseudo-atoms or pseudo-particles representing secondary chains or small parts of molecules. For instance, in a hydrocarbon chain, $\mathrm{CH}_{2}$ and $\mathrm{CH}_{3}$ groups can be replaced by pseudo-atoms [24]

Splitting the potential into bonded and non-bonded parts may make the implementation of a molecular dynamics simulation code easier, because a list of the neighboring atoms can be built when the system is set up. Such a list can be used to save computational time by just looking at listed neighbors of each atom when the system is perturbed. An additional division of the interatomic potential could be made based on the distance at which the interaction extends. The potential could be considered as representing short or long range interactions, having similar applicability than the division in bonded and non-bonded potentials [24].

\subsubsection{Initial and Boundary Conditions}

Setting up a system for molecular dynamics simulations implies the selection of a set of initial conditions, namely the positions and velocities of all the atoms in the system. The initial velocities of the atoms are assigned randomly in such way that the system has the desired temperature. As it will be shown later, the temperature of an atomic system is determined only by the velocity of the atoms. Additionally, the velocity of the atoms is corrected to ensure the overall momentum is zero, i.e. $\sum_{i=1}^{N} m_{i} v_{i}=0$ [23]. A common strategy to assign the initial velocities of the atoms is to set them following the Maxwell-Boltzmann distribution [23, 25], which is given by

$$
\rho\left(v_{i x}\right)=\left(\frac{m_{i}}{2 \pi k_{B} T}\right)^{\frac{1}{2}} \exp \left(-\frac{m_{i} v_{i x}^{2}}{2 k_{B} T}\right)
$$

where $\rho\left(v_{i x}\right)$ is the probability density for the velocity component $v_{i x}, m_{i}$ is the mass of the $i t h$ atom, $k_{B}$ is the Boltzmann constant, and $T$ is the targeted temperature of the system. A similar equation applies to the velocities along $\mathrm{y}$ and $\mathrm{z}$ axes. The Maxwell-Boltzmann distribution ensures that most of the atoms move with approximately the same speed, leaving only a few atoms with very high and very low speeds. It will be inconvenient for a simulation process to have a velocity distribution different than a unimodal for each type of atom, specially when having gradients of temperature is not the main purpose of the simulation.

The boundary conditions of the atomic system define how the atoms reaching the limits of the simulation box are treated. Three types of boundaries can be considered: i) free surfaces, where the atoms reaching the surface can remain there, diffuse through the surface, diffuse into the bulk 
of the system, or evaporate from it. The process followed by the atoms reaching the surface is determined by the energy they have when they reach the surface, ii) Reflecting surfaces, in this case the atoms reaching the surface bounce back into the bulk system, with or without energy loss due to the bouncing [25], and iii) Periodic boundaries, under these conditions the atoms reaching the limits of the simulation box are allowed to leave it, but they are reintroduced into the system, with the same velocity, through the opposite boundary [23]. Periodic boundary conditions are useful when the simulation is intended to investigate the bulk properties of the system, as it is the case in this research.

\subsubsection{Integration Algorithm for the Equations of Motion}

The algorithm used to integrate the equations of motion for the system of particles is another important part of the simulation process because it has a crucial effect on the performance of the program. The algorithm is expected to be accurate and stable in the description of the trajectory followed by the particles for long simulations, i.e. the trajectory of the particles is not highly affected by rounding errors. Also, the algorithm is expected to be conservative of the quantities that have been set to remain constant during the simulation (For example energy, temperature, pressure, etc), and able to run using large time steps to perform a small amount of recalculations of the interatomic forces. These recalculations can consume about $95 \%$ of the total CPU time for each evolution of the system [22, 26]. There are several numerical integration techniques that can be used to evolve the system from time $(t)$ to $(t+\Delta t)$. The simplest one is the Euler method, which in terms of the Hamiltonian of the system is

$$
p_{n+1}=p_{n}-\delta t * \frac{\partial \mathscr{H}\left(p_{n}, q_{n}\right)}{\partial q} \quad q_{n+1}=q_{n}+\delta t * \frac{\partial \mathscr{H}\left(p_{n}, q_{n}\right)}{\partial p}
$$

In this case by knowing all the coordinates $q_{i}$ and the momenta $p_{i}$ at the $n^{\text {th }}$ time step, the evolution to the $(n+1)^{\text {th }}$ time step can be easily calculated. This is a first-order, explicit scheme; but it can be also written in an implicit form requiring an iterative process to find the solution

$$
p_{n+1}=p_{n}-\delta t * \frac{\partial \mathscr{H}\left(p_{n+1}, q_{n+1}\right)}{\partial q} \quad q_{n+1}=q_{n}+\delta t * \frac{\partial \mathscr{H}\left(p_{n+1}, q_{n+1}\right)}{\partial p}
$$

By writing the Hamiltonian as the addition of kinetic and potential energies of the system, $\mathscr{H}=\frac{1}{2} p^{T}[M]^{-1} p+V(q)$, additional integration schemes can be written. Those schemes can be implicit and first-order in the velocity but explicit in the positions, and vice versa [22]. However, the Euler scheme for solving the equations of motion is not recommended because it is not time reversible and suffers a large energy drift [26].

A more sophisticate scheme known as Velocity Verlet has been developed, which is praised due to its long time stability. It has become widely used in modern molecular dynamics simulations codes [23]. The scheme involves an evaluation of the momenta at half of a time step using 


$$
p_{n+\frac{1}{2}}=p_{n}+\frac{1}{2 m} * \delta t * F\left(q_{n}\right)
$$

$F\left(q_{n}\right)$ is the interatomic force acting on each atom calculated given the positions in the $n^{\text {th }}$ time step. Using the momenta at half time step, the new positions are calculated for a full time step using

$$
q_{n+1}=q_{n}+\delta t * p_{n+\frac{1}{2}}
$$

Finally, the momenta at the end of the time step are given by

$$
p_{n+1}=p_{n+\frac{1}{2}}+\frac{1}{2 m} * \delta t * F\left(q_{n+1}\right)
$$

\subsubsection{Molecular Dynamics of Constrained Systems}

Constraint Dynamics is a MD technique developed to analyze the evolution of systems in which certain degrees of freedom, expressed as the distance between two particles, need to be constrained (Holonomic constraints). Systems in which the use of Holonomic constraints is useful are characterized by the presence of degrees of freedom that change with a rapid frequency but small amplitude of variation. This situation emerges usually during modeling of systems such as long hydrocarbon and polymeric chains [27, 28, 29], in which the vibrations of the $\mathrm{C}-\mathrm{H}$ bonds have a much higher frequency that those of the $\mathrm{C}-\mathrm{C}$ or other bonds; but, since they have a shorter amplitude, they can be modeled as rigid bonds. Similar situations may appear in biological systems involving modeling the folding and interaction of protein chains [30, 31].

The main idea behind the technique is to use a set of forces applied to the particles that need to be constrained. The set of constraint forces is added to the interatomic forces (obtained from the interatomic potential) to guarantee that the constraints are fulfilled at every stage of the evolution of the system [23].

The constraint forces are obtained from the set of Lagrangian multipliers calculated during the solution of the equations of motion. The mathematical formulation of the equations of motion for the constrained particles is given by

$$
m_{i} \ddot{\vec{r}_{i}}=\vec{f}_{i}+\vec{g}_{i}
$$

where $m_{i}$ is the mass of the particle $i, \ddot{\vec{r}}_{i}$ is the acceleration of the particle $i, f_{i}$ is the interatomic force exerted on the particle $i$, and $g_{i}$ is the constraint force applied to particle $i$ to guarantee the fulfillment of the constraints imposed.

The constraints are written in terms of the atomic positions as 


$$
\psi_{i j}=\left(r_{i j}\right)^{2}-\left(d_{i j}\right)^{2}=0
$$

where $\psi_{i j}$ is the constraint function, $r_{i j}$ is the current distance between particles $i$ and $j$, and $d_{i j}$ is the specified distance between particles $i$ and $j$.

The constraint force $\vec{g}_{i}$ is obtained from the constraint function as

$$
\vec{g}_{i}=\sum_{j} \sum_{k} \frac{1}{2} \lambda_{j k} \vec{\nabla}_{\vec{r}_{i}}\left(\psi_{j k}\right)
$$

where $\lambda_{j k}$ is the Lagrangian multiplier for the distance between particles $i$ and $j$, and $\vec{\nabla}_{\vec{r}_{i}}\left(\psi_{j k}\right)$ is the gradient of the function $\psi_{j k}$ relative to the position vector $\left.\vec{r}\right]$.

The solution of the set of equations given by Eq. 2.12 can be written as

$$
\vec{r}_{i}(t+\delta t)=\vec{r}_{i \mathrm{uc}}(t+\delta t)+\left(\frac{(\delta t)^{2}}{m_{i}}\right) \vec{g}_{i}(t)
$$

where $\vec{r}_{i}(t+\delta t)$ is the position vector of particle $i$ at the time step $(t+\delta t)$ fulfilling the imposed constraints, $\vec{r}_{i \mathrm{uc}}(t+\delta t)$ is the position vector of particle $i$ at the time step $(t+\delta t)$ if it moves unconstrained, $m_{i}$ is the mass of the particle $i, \delta t$ is the size of the time step, and $\vec{g}_{i}(t)$ is the constraint force on the particle $i$ at the time step $(t)$.

An ingenious method to solve the Eq. 2.12 was developed by Ryckaert et al. [27], and it is presented in an algorithm called SHAKE. The algorithm consists of four fundamental steps as follow

i. Calculate the unconstrained positions at the time step $(t+\delta t)$, i.e. $\vec{r}_{i \mathrm{uc}}(t+\delta t)$, by solving the equations of motion (Eq. 2.4) using only the forces due to the interatomic interactions. Any method can be used during this step (See section 2.1.4).

ii. Calculate the Lagrangian multipliers, and the constraint forces corresponding to them based on the unconstrained positions of the constrained particles. These forces correspond to $\vec{g}_{i}(t)$ in Eq. 2.15,

iii. Update the positions of the constrained particles according to Eq. 2.15.

iv. Update the velocities of the constrained particles by calculating the displacement, using $\vec{r}_{i \mathrm{uc}}(t+\delta t)$ and $\vec{r}_{i \mathrm{uc}}(t)$, and dividing it by the time step $\delta t$.

Ryckaert et al. 27] presented a form of calculating the Lagrangian multipliers, therefore the constraint forces, based on an iterative procedure using the constraint equation (Eq. 2.13). That procedure was adopted for this research and its specific implementation is described in Chapter 4.

\footnotetext{
${ }^{1} r_{i j}$ is the magnitude of the vector $\vec{r}_{i j}$ defined as $\vec{r}_{i j}=\vec{r}_{j}-\vec{r}_{i}$
} 


\subsection{Physical Properties of Atomic Systems}

\subsubsection{Temperature and Kinetic Energy of an Atomic System}

Neglecting the energy associated with rotation, the kinetic energy of an atomic system is given by the addition of the translational energies of the individual particles. $p$ being the generalized momenta of each particle, the kinetic energy of the system is given by

$$
\mathscr{K}=\sum_{i=1}^{N} \frac{\left|p_{i}\right|^{2}}{2 m_{i}}=\sum_{i=1}^{N} \sum_{\alpha=x, y, z} \frac{p_{i \alpha}^{2}}{2 m_{i}}
$$

The relation between the kinetic energy and the temperature is stated in the Virial theorem written in the form of generalized equipartition [32]. For each particle

$$
\left\langle p_{k} \frac{\partial \mathscr{H}}{\partial p_{k}}\right\rangle=k_{B} T
$$

where, $\langle\mathscr{A}\rangle$ means time average of the quantity $\mathscr{A}$, and $k_{B}$ is the Boltzmann's constant. Recalling that the Hamiltonian, $\mathscr{H}$, for each particle is given by

$$
\mathscr{H}=\sum_{\alpha=x, y, z} \frac{p_{i \alpha}^{2}}{2}+\sum_{j}^{N} V_{2}\left(r_{i j}\right)+\sum_{j}^{N} \sum_{k}^{N} V_{3}\left(r_{i j}, r_{i k}, r_{j k}\right)
$$

Then, the LHS of the Eq. 2.17 becomes

$$
\left\langle p_{k} \frac{\partial \mathscr{H}}{\partial p_{k}}\right\rangle=\left\langle\frac{p_{k}^{2}}{m}\right\rangle=2\langle\mathscr{K}\rangle
$$

When the whole system is considered $\langle\mathscr{K}\rangle$ represents the average of the kinetic energy of the entire system. And the RHS of the equation Eq. 2.17 becomes equal to $3 N k_{B} T$, because it must include the effect of the components of the momentum along the three coordinate axes for each of the $\mathrm{N}$ particles. Therefore, Eq. 2.17 is equal to

$$
2\langle\mathscr{K}\rangle=3 N k_{B} T
$$

Finally, from Eq. 2.20 the average temperature of the system can be derived as

$$
T=\frac{2\langle\mathscr{K}\rangle}{3 N k_{B}}=\frac{1}{3 N k_{B}}\left\langle\sum_{i}^{N} \sum_{\alpha} \frac{p_{i \alpha}^{2}}{m_{i}}\right\rangle
$$

When the instantaneous values of momenta are used the instantaneous temperature of the system is obtained.

\subsubsection{Pressure in an Atomic System}

The generalized equipartition form of the Virial theorem also states that [32] 


$$
\left\langle q_{k} \frac{\partial \mathscr{H}}{\partial q_{k}}\right\rangle=k_{B} T
$$

Averaging over all the particles and along the three Cartesian coordinates the RHS of the equation Eq. 2.22 becomes equal to $3 N k_{B} T$. The LHS can be developed using

$$
\frac{\partial \mathscr{H}}{\partial q_{k}}=-\dot{p_{k}}=\nabla_{k} V=-f_{k}
$$

Therefore, Eq. 2.22 can be rewritten as

$$
\left\langle q_{k} \frac{\partial \mathscr{H}}{\partial q_{k}}\right\rangle=-\left\langle q_{k} f_{k}\right\rangle=3 N k_{B} T
$$

where $f_{k}$ is the total force acting on each particle. Such a force is composed of an internal part, coming from interatomic interactions, and an external part representing the effect of a container or the environment. Hence the Eq. 2.24 can be expanded as

$$
-\left\langle q_{k} f_{k}\right\rangle=-\left\langle q_{k} f_{k}^{i n t}\right\rangle-\left\langle q_{k} f_{k}^{e x t}\right\rangle=3 N k_{B} T
$$

The pressure in the atomic system is related to the external force, $f_{k}^{e x t}$, and the volume, $\mathscr{V}$, of the system by

$$
-\frac{1}{3}\left\langle q_{k} f_{k}^{e x t}\right\rangle=P \mathscr{V}
$$

The component representing the effect of the interatomic forces is called internal Virial of the system and is given by

$$
w=\frac{1}{3} \sum_{i=1}^{N-1} \sum_{j>i}^{N} r_{i j} f_{i j}
$$

$r_{i j}$ and $f_{i j}$ being the position vector and the force vector between the particles $i$ and $j$, respectively.

Finally, by using Eqs. 2.24, 2.26, and 2.27 an expression for the pressure in the atomic system can be obtained as

$$
P \mathscr{V}=N k_{B} T+\langle w\rangle
$$

Instead of the averages, the instantaneous conditions can be used defining an instantaneous pressure for the system. The average of that pressure must be equal to $\mathrm{P}$ given by equation Eq. 2.28 , 


\subsubsection{Stress in Atomic Systems}

Combining Eqs. 2.20 and 2.24 a most common form of the Virial theorem is obtained, which states a direct relation between the kinetic and potential energies of the system as

$$
2\langle K\rangle=-\left\langle q_{k} f_{k}\right\rangle=\left\langle q_{k} \nabla V\right\rangle
$$

Eq. 2.29 can also be derived following a procedure presented by Goldstein [33]. It starts by considering the time variation of a certain quantity, $G$, defined as

$$
G=\sum_{i} p_{i} q_{i}
$$

where, $p_{i}$ and $q_{i}$ represent the momentum and the coordinate of each particle. Then, the derivative of the function $\mathrm{G}$ with respect to time is given by

$$
\frac{d G}{d t}=\sum_{i} \dot{p}_{i} q_{i}+\sum_{i} p_{i} \dot{q}_{i}=\sum_{i} \dot{p}_{i} q_{i}+2 K
$$

If the quantity $\frac{d G}{d t}$ is integrated over a time $\tau$, assuming all the coordinates and momenta remain finite, there is an upper bound for $G$ and $\int_{0}^{\tau} \frac{d G}{d t} d t=G(\tau)-G(0)$. By selecting $\tau$ to be long enough the integral can be as small as one wants, particularly zero. A similar conclusion is obtained if the motion of all the particles in the system is periodic and $\tau$ is selected to be the period. Therefore, Eq. 2.31 becomes

$$
2\langle K\rangle+\left\langle\sum_{i} \dot{p}_{i} q_{i}\right\rangle=0
$$

Using $\dot{p}_{i}=f_{i}$, Eq. 2.32 becomes identical to Eq. 2.29, Finally, using the same arguments used to decompose $\left\langle q_{i} f_{i}\right\rangle$ in the Eq. 2.25 the Eq. 2.28 can be reproduced.

The procedure presented by Goldstein in [33] is an alternative way to derive the Virial theorem and an expression for the pressure in the system. That procedure can be generalized to determine a tensorial quantity, $\prod_{\alpha \beta}$, considered as a form of stress tensor for the atomic system. The generalization of Goldstein's procedure is presented by McLellan [34]. It follows the same steps presented in [33] but using a tensorial quantity defined as: $G_{\alpha \beta}=\sum_{i} \sum_{j} q_{i}^{\alpha} p_{j}^{\beta}$. An equation similar to Eq. 2.28 is obtained as

$$
\Omega \prod_{\alpha \beta}=-\left\langle\sum_{i} \sum_{j} \frac{1}{m}\left(q_{i}^{\alpha} p_{j}^{\beta}\right)\right\rangle+\left\langle\sum_{i} \sum_{j} q_{i}^{\alpha} f_{i}^{\beta}\right\rangle
$$

where $\Omega$ is the volume over which the averages are being computed, and $\prod_{\alpha \beta}$ represents a stress tensor. The first term of the RHS in Eq. 2.33 is the stress caused by momentum in direction $\beta$ transfered in direction $\alpha$, and across the surface of the volume $\Omega$. The second term is the stress generated by interatomic forces exerted between the atoms inside the volume. This last term is 
commonly written as in Eq. 2.27. Zhou [35] demonstrated that this second component of the stress tensor derived from the Virial Theorem corresponds exactly to the Cauchy stress of the system. Accordingly, the Cauchy stress used in this research is written as

$$
\Omega \sigma_{\alpha \beta}=\left\langle\sum_{i} \sum_{j} q_{i}^{\alpha} f_{i}^{\beta}\right\rangle=\left\langle\frac{1}{3} \sum_{i}^{N-1} \sum_{j}^{N}\left(r_{i j}^{\alpha} f_{i j}^{\beta}\right)\right\rangle
$$

\subsubsection{Structural Properties of Atomic Systems}

Describing the structure of an atomic system consists of defining functions to determine the relative distances between the different atoms, their bonding angles, and the general arrangement of the atoms in the structure. Those functions allow determining values that can be compared to experimental measures. One of the most used structural properties for atomic systems is the Pair Distribution Function (PDF). It is a function defined by the likelihood of finding a pair of atoms separated by a distance $r$ in the actual structure, divided by the likelihood of finding two atoms separated by the same distance $r$ in a structure that has a uniform distribution and the same density as the actual structure [23, 26]. The PDF is commonly used because its Fourier transform, called Static Structure Factor, can be directly compared to neutron scattering results [36, 37]. Additionally, both bonding and non-bonding neighbors distances directly obtained from the PDF are comparable to experimental information. The PDF is given by

$$
g_{\alpha \beta}\left(r_{1}, r_{2}\right)=\frac{V^{2}}{N_{\alpha} N_{\beta}}\left\langle\sum_{i \in\{\alpha\}}^{N_{\alpha}} \sum_{j \in\{\beta\}}^{N_{\beta}} \delta\left(r_{1}-r_{i}\right) \delta\left(r_{2}-r_{j}\right)\right\rangle
$$

or

$$
g_{\alpha \beta}\left(r_{1}, r_{2}\right)=\rho_{\alpha}^{-1} \rho_{\beta}^{-1}\left\langle\sum_{i \in\{\alpha\}}^{N_{\alpha}} \sum_{j \in\{\beta\}}^{N_{\beta}} \delta\left(r_{1}-r_{i}\right) \delta\left(r_{2}-r_{j}\right)\right\rangle
$$

where, $\mathrm{V}$ is the volume of the system, $\mathrm{N}_{\alpha}$ and $\mathrm{N}_{\beta}$ are the number of particles of the entities of type $\alpha$ and $\beta$, respectively, $\rho_{\alpha}$ and $\rho_{\beta}$ are the corresponding densities of the $\alpha$ and $\beta$ subsystems, and the symbol \langle\rangle means ensemble average. The ensemble average is used when several configurations need to be considered for the analysis structure. The term inside the \langle\rangle symbol indicates that when finding the distribution of distances between the atoms of the entities type $\alpha$ and $\beta$ one must locate each atom of type $\alpha$ and obtain the distance from it to each one of the atoms of type $\beta$.

In Fig. 2.1 a plot of the PDF for a silica aerogel with density of about $0.45 \frac{\mathrm{g}}{\mathrm{cm}^{3}}$ is shown. The peaks appearing at distances smaller than $5 \AA$ correspond to the nearest neighbors, for instance the peak around $1.6 \AA$ represents the bonding distance between the silicon and oxygen atoms [36]. A straight forward procedure to determine the PDF for a system uses the following steps:

i. For the selected configuration, build a histogram of the distances between the atoms in the structure. If several configurations are used to characterize the structure, a histogram must 


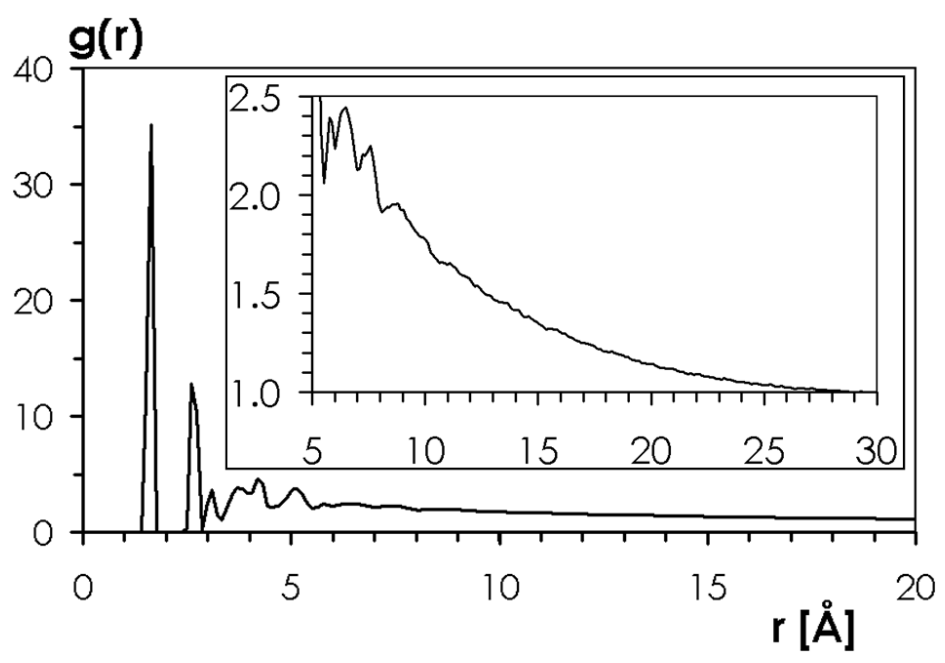

Figure 2.1: Example of the Pair Distribution Function for a silica aerogel with density of $0.45 \frac{\mathrm{g}}{\mathrm{cm}^{3}}$.

be created for each configuration. The usage of several configurations may become necessary when the positions of the particles change rapidly (system with high kinetic energy) and the PDF of the average configuration is the function of interest.

ii. If several configurations are used an average histogram must be created by adding the data in all histograms and dividing by the number of configurations used. If only one configuration is chosen this step is not necessary.

iii. Create a histogram for a configuration having the same density as the actual sample, but in which the atoms are uniformly distributed across the volume of the system. This is achieved by considering a region around a distance $r$ and multiplying the volume of that region by the total number of atoms divided by the total volume occupied by the sample.

iv. Calculate the PDF by dividing the data in the histogram created in step ii) by the histogram created in step iii).

Aerogels and xerogels are fractal structures [8, 11, 38, 39]; therefore an atomistic model to investigate these type of materials must reproduce their fractal properties. The fractality of a structure is characterized by a non-integer number called fractal dimension. It indicates how the volume of the fractal varies with respect to its linear scale. For example, if a fractal is enclosed in a cubic box, the volume of the box is related to its linear scale (the length of the side) by an expression of the form $V \propto L^{D}$, where $\mathrm{D}$ is the Euclidean dimension of the box and is equal to 3. For a fractal the relation between the volume and the linear dimension is the same; but the exponent $\mathrm{D}$, which is called fractal dimension and denoted $d_{f}$, is smaller than 3 [40]. Among the different procedures that can be used to determine the fractal dimension of an atomic system two are considered in this research. One based on the pair distribution function, and the other one based on the modeling of a scattering experiment. 


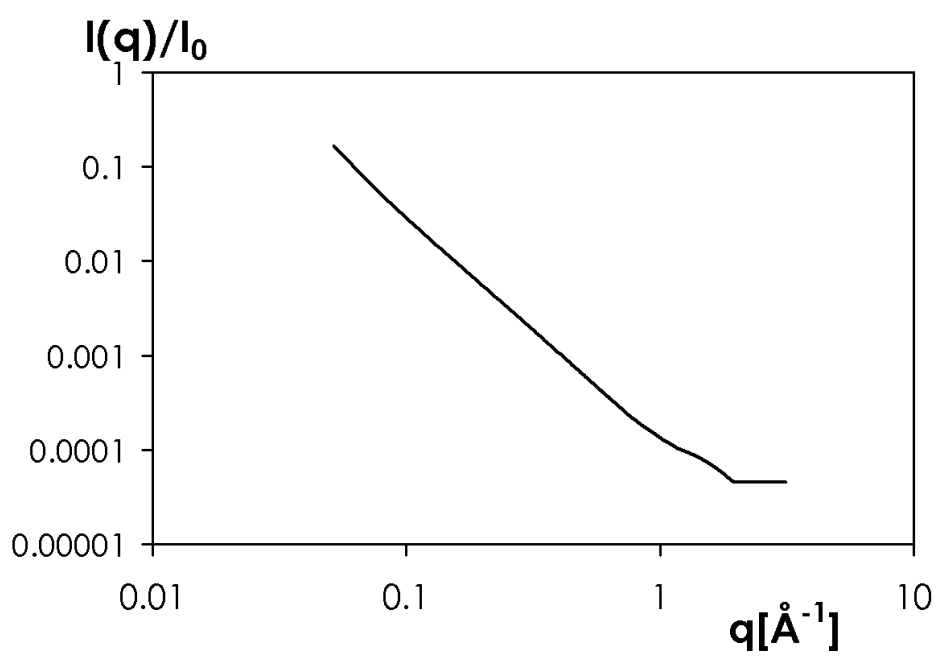

Figure 2.2: Example of scattering intensity vs. wavenumber plot for a silica aerogel with density of about $0.45 \frac{\mathrm{g}}{\mathrm{cm}^{3}}$.

In the inset of Fig. 2.1 the region of the PDF beyond $5 \AA$ is enlarged. From the plot, it can be seen that the value of PDF continuously decreases to one. That decay can be used to estimate the fractal dimension of porous systems according to the relation below, where $g(r)$ is the PDF, [4]

$$
d_{f}=3+\frac{\mathrm{d}(\operatorname{Ln}(g(r)))}{\mathrm{d}(\operatorname{Ln}(r))}
$$

The other procedure that is considered here to obtain the fractal dimension of the samples is based on a simulated scattering experiment [42, 43]. To simulate a scattering experiment it is necessary to calculate the scattering intensity corresponding to different wavenumbers. An expression to calculate the scattering intensity when the positions of all the particles in the system are known is given by [44, 45]

$$
\frac{I(q)}{I_{0}}=\sum_{i j} \frac{\sin \left(q r_{i j}\right)}{q r_{i j}}
$$

where $I$ is the scattering intensity, $I_{0}$ is a reference value of intensity, $q$ is the wavenumber and $r_{i j}$ is the distance between two atoms $i$ and $j$.

For fractal structures, the scattering intensity can be related to the wavenumber by a power law, where the exponent is the negative of the fractal dimension of the structure, i.e. $I \propto q^{-d_{f}}$ [44, 45]. An example of the $I(q)$ is plotted in the Fig. 2.2. For large wavenumbers the scattering intensity corresponds to the individual particles and remains constant; while for small wavenumbers, clusters of particles are responsible for the scattering. The limiting value of wavenumber in the scattering is determined by the size of the sample modeled. For example, for a silica aerogel sample with density of $0.45 \frac{\mathrm{g}}{\mathrm{cm}^{3}}$ the fractal dimension is about 2.4 . 


\section{Chapter 3}

\section{Molecular Dynamics Simulation Models for Silica Aerogels and Xerogels}

In this chapter the use of Molecular Dynamics (MD) simulations to model and characterize aerogels and xerogels is presented. Most of the computational models developed for aerogels and xerogels are devoted to the study of the characteristics of the structure and its formation; this is addressed in section [3.1. Section 3.2 covers the most relevant work related to the use of MD to model the mechanical behavior of silica aerogels and xerogels. In section 3.3 the interatomic potential chosen to model the silica samples for this research is discussed. The interatomic potential was developed by Vashishta et al. [36, 46]. It is composed of two-body and three-body functions accounting for the energy associated with the stretching and bending of covalent $\mathrm{Si}-\mathrm{O}$ bonds. In section 3.4 the modeling technique adopted for this research is introduced, along with the results obtained from models for dense silica glass. The model for silica glass was used to determine the simulation parameters necessary to model silica aerogel and xerogel samples. Section 3.5 describes the application of the modeling technique to the study of silica aerogels and xerogels. The samples produced display fractal features in the intermediate range of structural arrangement. The fractal dimension and its relationship with the density of the samples are in agreement with experimental data. The mechanical characterization was done simulating uniaxial tension tests. The elastic modulus and the strength of the samples relate to the density following a power law. This type of relationship has been found experimentally, not only for silica aerogels and xerogels but also for other types of fractal systems, as discussed earlier in section 1.2.2. 


\subsection{Models of the Structure of Silica Aerogels and Xerogels}

MD simulations have been one of the most used techniques to model the structural characteristics of silica aerogels and xerogels. MD models implemented to study silica aerogels and xerogels use interatomic potentials developed for dense silica glass and preparation procedures to recreate the porous structure of those materials. In this section a review of published work about the use of MD simulations for modeling the structure of aerogels and xerogels is presented.

Kieffer et al. [41 prepared computational samples of aerogels and xerogels modeling a dense glass, then gradually expanding the volume of the sample keeping the number of particles (atoms) constant. The interatomic potential accounts for two-body interactions only. The samples had densities varying from $0.2 \frac{\mathrm{g}}{\mathrm{cm}^{3}}$ to $2.2 \frac{\mathrm{g}}{\mathrm{cm}^{3}}$ (density of dense silica glass), and were created using 1500 atoms. During the expansion the density of the samples is reduced forcing atomic rearrangement, causing the $\mathrm{Si}-\mathrm{O}$ bonds to break, and leading to the formation of fractal structures. The resulting fractal structures were characterized by a fractal dimension that changes linearly with the density for samples with densities lower than $1 \frac{\mathrm{g}}{\mathrm{cm}^{3}}$. The fractal dimension of the samples varied between 2.0 and 3.0 .

Nakano et al. [47] used the interatomic potential developed by Vashishta et al. [36, 46], described in section 3.3, to model porous silica with densities varying from $0.2 \frac{\mathrm{g}}{\mathrm{cm}^{3}}$ to $2.2 \frac{\mathrm{g}}{\mathrm{cm}^{3}}$ in a system with 41472 atoms. Similarly to Kieffer et al. [41, Nakano et al. 47] gradually expanded the system until the desired density was obtained. They investigated the dependence of the fractal dimension, internal surface area, the pore to volume ratio, pore size distribution, correlation length, and mean particle size on the density of the sample. Kieffer et al. 41 and Nakano et al. [47. obtained the fractal dimension of the samples from the pair distribution function (PDF)(see section 2.2.4).

Pohl et al. 48 used different computational techniques to generate porous silica samples, and to compare the structural properties obtained from the models with those determined experimentally. One of the computational techniques used in [48] to generate porous samples followed the same procedure as Kieffer et al. [41] and Nakano et al. [47]; but the interatomic potential chosen was the one developed by Feuston and Garofalini [49]. They found that the variation of the pore size distribution with the density for the simulated samples compares well to experimental results. However, they also concluded that although samples of silica aerogel generated by expanding a glass allow matching certain experimental results, the computational representation fails to match the chemical composition of real aerogels. It contains a stoichiometric ratio of silicon atoms to oxygen atoms, and does not capture the formation of hydroxyl groups (-OH) on the surface of the material. In spite of the observations made by Pohl et al. 48 MD simulations of aerogels and xerogels have been successfully used to investigate the most important properties of these materials. 
Campbell et al. [50, also using Vashishta et al.'s potential [36, 46], generated porous samples by placing spherical clusters of dense silica glass in a large volume and sintering the system at constant pressure and temperature. With that approach they generated samples of densities varying from $1.67 \frac{\mathrm{g}}{\mathrm{cm}^{3}}$ to $2.2 \frac{\mathrm{g}}{\mathrm{cm}^{3}}$, a range of density corresponding to xerogels, allowing the study of the changes on the short-range and intermediate-range order of the structure with density.

Recently, Bhattacharya and Kieffer [51] used MD to study the formation of aerogels focusing on both the condensation and the water-forming reactions of fully hydrolyzed precursor molecules. This is the final stage of the gelation of a solution where the fully hydrolyzed molecules condense forming Si-O-Si bonds and releasing water molecules. The fractal dimension of the structures generated using this procedure are smaller than the one of structures generated by gradually breaking a dense glass as in Refs. [41] and [47. However, the relationship between fractal dimension and density is similar in both cases. The difference in the fractal dimension is attributed to the formation of structures with more uniform pore distribution compared to those produced by gradually rupturing a glass sample.

Although the procedures described so far reproduce results comparable to experimental data, they differ from each other and neither conclusively describes the processes that lead to formation of aerogel and xerogel structures. Developing additional procedures to create computational samples of aerogels and xerogels could provide supplementary insight into the formation and properties of fractal structures in general.

\subsection{Models of the Mechanical Properties of Silica Aerogels and Xerogels}

MD simulations have proven to be an effective tool to investigate the properties of silica aerogels and xerogels. However, the study of the mechanical properties of these materials have been mainly done via experimentation as it is presented in chapter 1. Campbell et al. [50, along with the study of the structural properties of xerogels, modeled the relation between elastic modulus, bulk modulus, shear modulus, and density. Their findings are in good agreement with experimental data predicting a power law relation between those properties and the density [5, 8, 10, 12, 52.

Other computational techniques have also been used to study the relation between elastic properties of aerogels and density producing results that compare well with experimental findings. For instance, Gelp [53] used a coarse-grained model in which spheres representing silica clusters were allowed to interact based on two potentials, a weak non-bonding potential modeled with a LenardJones form and a short-range bonding potential based on Morse's potential, including bending and torsion interactions for the links between the spheres. Ma et al. 54 modeled aerogel samples using a diffusion limited cluster-cluster aggregation algorithm combined with an algorithm to reduce the 
number of dead-ends formed in the structure. Using finite elements, they found that bulk modulus and density are related by a power law characterized by an exponent of 3.6, a result similar to those obtained experimentally and by MD simulations.

The MD simulation models used to study the mechanical properties of silica aerogels and xerogels have been limited to small ranges of densities. The present research involves the creation of computational samples with densities spanning from aerogels to dense silica ( 0.23 to $\left.2.2 \frac{\mathrm{g}}{\mathrm{cm}^{3}}\right)$. The samples are used to estimate a relationship between elasticity and strength over a wide range of densities.

In the next section the steps followed in this research to produce and test samples of aerogels and xerogels are presented.

\subsection{Interatomic Potential for Silica}

The interatomic potential chosen for this study was developed by Vashishta et al. [36, 46]. It is used because it accurately reproduces the structural parameters for dense silica glass. Using the structural factor $R_{\chi}$ introduced by Wright [37] to compare the experimental results of neutron scattering to computer simulations based on this potential produces a discrepancy of only $4.4 \%$, which is the smallest among all the available potentials for silica [50]. Vashishta's potential also excels in the representation of the elasticity of silica glass. The elastic modulus of the silica glass calculated using this potential has been reported to be around $70 \mathrm{GPa}$ [50], which compares well with the experimental value of $71.9 \mathrm{GPa}$ reported by Muralidharan et al. [55].

Vashishta's potential [36, 46] involves terms representing the interaction between two atoms (two-body component), which, disregarding if the atoms form a bond or not, accounts for the potential energy due to the distance between them. The potential also includes the potential energy due to the change of orientation and bonding angle of triplets of atoms (three-body component). The interatomic potential, along with the two-body and three-body components of the potential, are given by

$$
\begin{gathered}
V_{i j}=\sum_{i} \sum_{j} V_{i j}^{(2)}+\sum_{i} \sum_{j} \sum_{k} V_{i j k}^{(3)} \\
V_{i j}^{(2)}=\frac{H_{i j}}{r_{i j}^{\eta_{i j}}}+\frac{Z_{i} Z_{j}}{r_{i j}} \exp \left(\frac{-r_{i j}}{r_{1 s}}\right)-\frac{P_{i j}}{r_{i j}^{4}} \exp \left(\frac{-r_{i j}}{r_{4 s}}\right) \\
V_{j i k}^{(3)}=B_{j i k} f\left(r_{i j}, r_{i k}\right)\left(\cos \theta_{j i k}-\cos \tilde{\theta}_{j i k}\right)^{2}
\end{gathered}
$$

The RHS of Eq. 3.2, the two-body potential, is composed of three terms, namely steric repulsion due to the atomic size, screened Coulomb interactions between the atoms accounting for the electric 
Molecular Dynamics Simulation Models for Silica Aerogels and Xerogels

\begin{tabular}{lcccc}
\hline Potential & $\sigma[\mathbf{G P a}]$ & Deviation & E[GPa] & Deviation \\
\hline BMH potential [55] & $30-65$ & $67 \%-261 \%$ & 220 & $206 \%$ \\
\hline S-potential [55] & $24-35$ & $33 \%-49 \%$ & 220 & $206 \%$ \\
\hline BKS potential [55] & $15-22$ & $17 \%-22 \%$ & 100 & $39 \%$ \\
\hline FG potential [55] & $12-21$ & $33 \%-14 \%$ & 125 & $74 \%$ \\
\hline Vashishta's potential [50] & - & - & 70 & $2.6 \%$ \\
\hline Exp. data [55] & 18 & - & 71.9 & - \\
\hline
\end{tabular}

Table 3.1: Elastic modulus, E, and strength, $\sigma$, of silica glass obtained using different potentials

charge transfer, and charge-dipole interactions taking into account the electric polarizability of the atoms, respectively.

In Eq. 3.2 and Eq. 3.3, $r_{i j}$ is the distance between atoms $i$ and $j$. The parameters $H_{i j}$ and $\eta_{i j}$ are related to the strength and extension of steric repulsion, respectively. $H_{i j}$ can be expressed in terms of the ionic radii, $\sigma_{i}$ and $\sigma_{j}$, of the two atoms involved and an empirical parameter, $A_{i j}$. That relation is given by

$$
H_{i j}=A_{i j}\left(\sigma_{i}+\sigma_{j}\right)^{\eta_{i j}}
$$

The second term in the RHS of Eq. 3.2 involves the screened Coulomb interactions between the atoms, and accounts for the electric charge transfer. The quantities $Z_{i}$ and $Z_{j}$ are the effective charge of the $i^{t h}$ and $j^{t h}$ ions. Their values are obtained from electronic structure calculations done using Quantum Mechanics.

The third term in the RHS of Eq. 3.2 accounts for the charge-dipole interactions considering the electric polarizability, $\alpha_{i}$, of the atoms. The factor $P_{i j}$ is given by

$$
P_{i j}=\frac{1}{2}\left(\alpha_{i} Z_{j}^{2}+\alpha_{j} Z_{i}^{2}\right)
$$

The three-body part of the potential, presented in Eq. 3.3, is always positive to prevent bonding angles deviating from the ideal values. $B_{j i k}$ is the strength of the three-body interaction, $\theta_{j i k}$ is the angle between the vector positions of the atoms, i.e. $\vec{r}_{i j}$ and $\vec{r}_{i k}$ with $i$ being the center atom, and $\tilde{\theta}_{j i k}$ is the ideal bond angle. The function $f$ represents the effect of bond stretching and is given by

$$
f\left(r_{i j}, r_{i k}\right)=\exp \left(\frac{l}{r_{i j}-r_{c 3}}+\frac{l}{r_{i k}-r_{c 3}}\right)
$$

where $l$ and $r_{c 3}$ are empirical parameters.

Although, there are six possible three-body interactions in the system, this potential only considers the most dominant ones, which are related to (Si-O-Si) and (O-Si-O) angles [36]. 
Other important potentials for silica that have been developed and used to study the structure and mechanical properties of silica glass are the Born-Meyer-Huggins potential (BMH potential) [55], which is a two-body potential of the form

$$
V_{i j}=\frac{q_{i} q_{j}}{r_{i j}}+A_{i j} \exp \left(-b_{i j} r_{i j}\right)+C_{i j} r_{i j}^{-6}+D_{i j} r_{i j}^{-8}
$$

where, $r_{i j}$ is the distance between the pair of atoms, and the parameters $A_{i j}, b_{i j}, C_{i j}$ and $D_{i j}$ are empirical constants obtained by fitting simulated data to structural information obtained experimentally.

The Soules potential (S-potential) [56] is a two-body potential based on the BMH potential. It is given by

$$
V_{i j}=A_{i j} \exp \left(\frac{-r_{i j}}{\rho}\right)+z_{i} z_{j} e^{2} \frac{\left(1-\operatorname{erf}\left(\frac{r_{i j}}{\eta L}\right)\right)}{r_{i j}}-\frac{\left(z_{i}^{2}+z_{j}^{2}\right) e^{2}}{\sqrt{\pi} \eta L}
$$

where, $r_{i j}$ is the distance between the pair of atoms, $z_{i}$ is the electronic charge of the $i^{\text {th }}$ ion, $\eta$ is the number of valence shell electrons, $L$ is the size of the simulation box, and $A_{i j}$ and $\rho$ are constants obtained by fitting experimental data.

van Beest, Kramer and van Sauten [57] developed an interatomic potential, BKS potential, deriving the parameters from a combination of ab-initio calculations and experimental data. The potential is given by

$$
V_{i j}=\frac{z_{i} z_{j}}{r_{i j}}+A_{i j} \exp \left(-b_{i j} r_{i j}\right)-\frac{c_{i j}}{r_{i j}^{6}}
$$

where $r_{i j}$ and $z_{i}$ have the same meaning that in the previously describe potentials, and $A_{i j}, b_{i j}$ and $c_{i j}$ are the constant parameters.

Two-body potentials as the ones described above are based on the ionic characteristics of the Si-O bond, and adequately reproduce some of the structural features of silica glass related with the bonding distances. However, they fail to properly reproduce the angle distribution of the Si-O-Si and O-Si-O bonds. That drawback is corrected by the addition of directionally dependent threebody potentials, which describe the covalent characteristics of the Si-O bond [58].

Feuston and Garafalini [58] derived a potential (FG potential) based on the BMH potential adding a three body potential. The two-body component of this potential is given by

$$
V_{i j}=A_{i j} \exp \left(\frac{-r_{i j}}{\rho}\right)+\frac{\left(z_{i} z_{j} e^{2}\right)}{r_{i j}} \operatorname{erfc}\left(\frac{r_{i j}}{\beta_{i j}}\right)
$$

where $A_{i j}, \rho$ and $\beta_{i j}$ are parameters. The three-body component added to the potential is given by 


$$
V_{i j k}=h\left(r_{i j}, r_{i k}, \theta_{j i k}\right)+h\left(r_{j i}, r_{j k}, \theta_{k j i}\right)+h\left(r_{k i}, r_{k j}, \theta_{j k i}\right)
$$

and,

$$
h\left(r_{i j}, r_{i k}, \theta_{j i k}\right)=\left\{\begin{array}{llll}
\lambda_{i} \exp \left(\frac{\gamma_{i}}{r_{i j}-r_{i}^{c}}+\frac{\gamma_{i}}{r_{i k}-r_{i}^{c}}\right)\left(\cos \theta_{j i k}-\cos \theta_{j i k}^{c}\right) & , r_{i j}<r_{i}^{c} & \text { and } & r_{i k}<r_{i}^{c} \\
0 & , r_{i j} \geq r_{i}^{c} & \text { or } & r_{i k} \geq r_{i}^{c}
\end{array}\right.
$$

where $\lambda_{i}, \gamma_{i}, r_{i}^{c}$, and $\theta_{j i k}^{c}$ are constants, and $\theta_{j i k}$ is the angle formed by vectors $\vec{r}_{i j}$ and $\vec{r}_{i k}$. The parameters for this potential are derived by fitting experimental data and numerical results.

Muralidharan et al. [55] report the values of the mechanical properties of silica glass obtained using the potentials mentioned above. Those values and their deviation with respect to experimental data are presented in Table 3.1. It is worthy recalling that Vashishta's potential leads to a elastic modulus of about $70 \mathrm{GPa}$, which deviates from the experimental data by only $2.6 \%$.

\subsection{Model of Dense Silica Glasses}

In this section the steps necessary to produce and characterize computational samples of dense silica are discussed, namely the creation of amorphous structures starting from crystalline samples, methods of applying displacement fields for simulating tension tests, and maintaining isothermal conditions during the simulation of a tension test. This section corresponds to the work presented by the author at the 2008 ASME International Mechanical Engineering Congress and Exposition [59].

\subsubsection{Sample Preparation and Structural Characterization}

To model silica glass, an amorphous form of silica, there are two common ways to define the initial atomic positions. The first consists of setting the atoms at the atomic positions corresponding to a crystalline form of silica (the atomic positions for crystalline silica are reported elsewhere [60]), then model the heating of the system until its temperature is above the melting point, and quenching the liquid. Heating up and cooling down the atomic system is achieved by controlling the speed of the particles representing the atoms. In order to heat up the system the speed of the atoms is increased and to cool down the system the speed of the atoms is reduced. The kinetic energy of the atoms and the temperature of the system are related according to Eq. 2.21. The fast cooling during the quenching step prevents the system from recrystallizing and leads to the formation of a structure that no longer has long-range order, only short-range order (up to first neighbors) is preserved. The second method to produce an amorphous sample is by randomly distributing silicon and oxygen atoms in the simulation box, continuing by heating up and quenching the system. The 


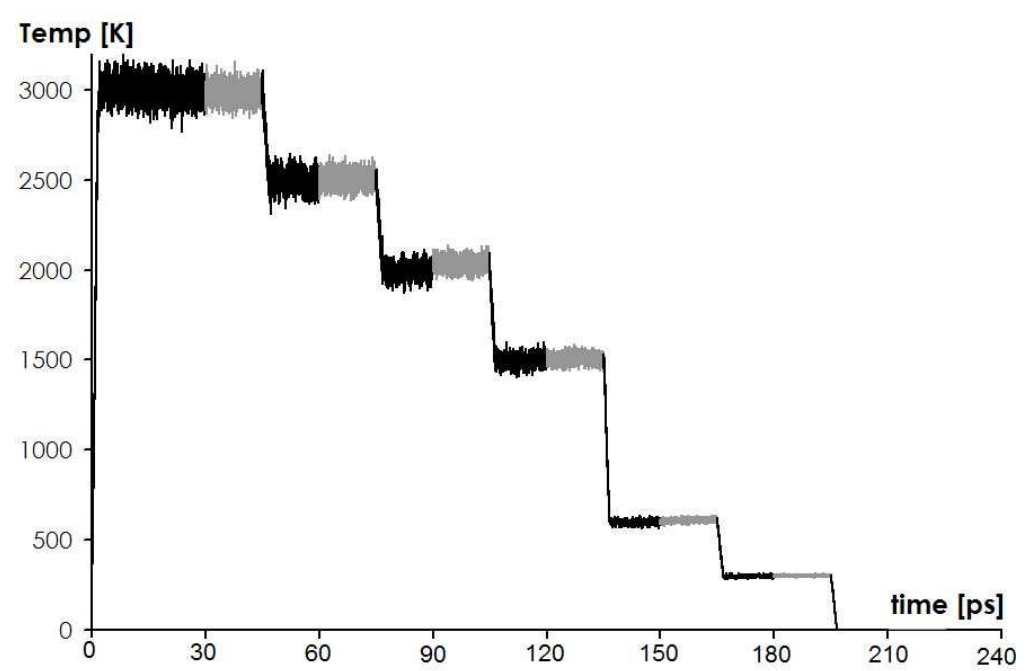

Figure 3.1: Temperature evolution during the creation of a sample of amorphous silica. Black lines correspond to NVT ensemble simulation, gray lines correspond to NVE ensemble simulation

method selected for this research is based on the former procedure. The samples representing dense silica glass are prepared starting from a sample of $\beta$-cristobalite, a phase of silica having a density similar to dense silica glass $\left(2.2 \frac{\mathrm{g}}{\mathrm{cm}^{3}}\right)$.

The goal is to create a fully randomized structure. The initial system can be setup with atoms at the positions corresponding to any given crystalline structure or randomly distributed in the simulation box. Then, the system must be heated up to a high temperature simulating the liquid state, and then cooled down using high cooling rates to avoid recrystallization. The cooling can be done either directly from the liquid phase to the desired temperature or in a step-wise process, thermalizing the system at different temperatures [36, 61].

The procedure followed in this research is similar to that used by Vashishta et al. [36]. A system composed by 2160 atoms ( $720 \mathrm{Si}$ atoms and $1440 \mathrm{O}$ atoms) sizing $71.7 \times 21.5 \times 21.5 \AA^{3}$ is set up with the atoms placed at the crystalline positions corresponding to $\beta$-cristobalite, a crystalline phase of silica having the same density as amorphous silica. The positions of the atoms for the $\beta$-cristobalite system are given in Ref. [60]. Periodic boundary conditions are applied along the three Cartesian directions and the time step is selected as $0.5 \mathrm{fs}$. The crystal is heated up to $3000 \mathrm{~K}$ and is kept at that temperature for $30 \mathrm{ps}$ (60,000 time steps of the simulation) under a temperature-controlled condition (NVT ensemble). Subsequently, the temperature control is removed and the system is allowed to stabilize without further heat transfer, under adiabatic conditions (NVE ensmble), for 15 ps. After that period, the system is cooled to $2500 \mathrm{~K}$ at a cooling rate of about $800 \frac{K}{p s}$ and kept under temperature-controlled conditions for $15 \mathrm{ps}$ before removing the temperature control and thermalizing for additional $15 \mathrm{ps}$. The same scheme of cooling and thermalizing was used to create systems at 2000,1500, 600, 300 and $0 \mathrm{~K}$. The evolution of the temperature and the total 


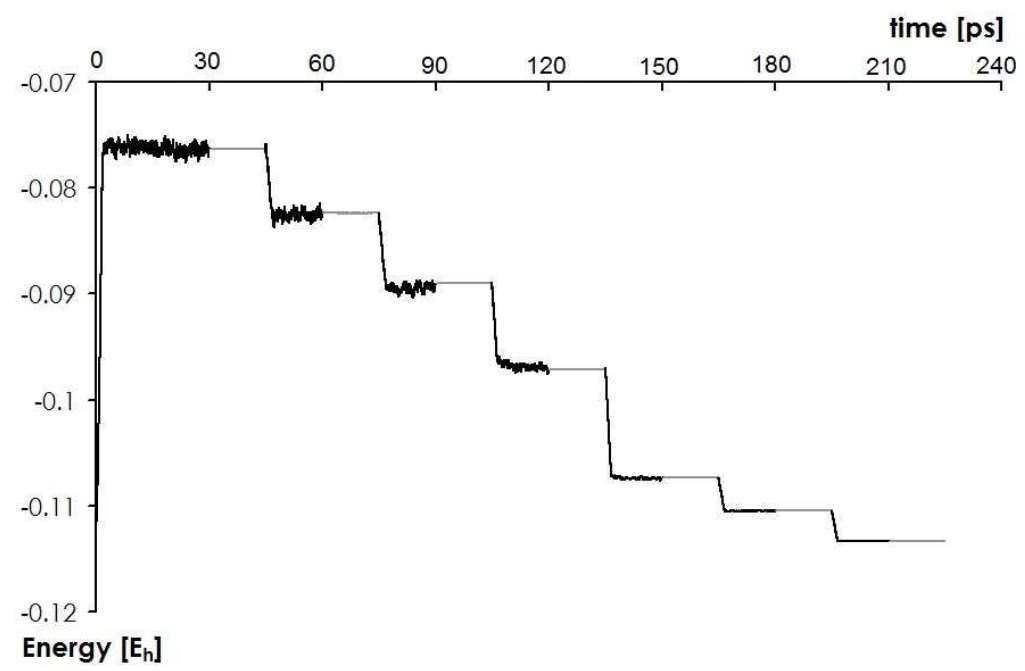

Figure 3.2: Total energy per particle during the creation of a sample of amorphous silica. Black lines correspond to NVT ensemble simulation, gray lines correspond to NVE ensemble simulation

energy per particle 1 for the system through the simulation are shown in Figures 3.1 and 3.2

The amorphization of the structure is verified by comparing the Pair Distribution Function (PDF) of the different atomic pairs, namely $\mathrm{Si}-\mathrm{O}, \mathrm{Si}-\mathrm{Si}$, and $\mathrm{O}-\mathrm{O}$, at the initial state ( $\beta$-cristobalite) with those for the systems at 300 and $0 \mathrm{~K}$. The comparison of the PDF for Si-O distances for $\beta$ cristobalite and amorphous samples at 300 and $0 \mathrm{~K}$ is shown in the Fig. 3.3. The Si-O distances peaks around $1.6 \AA$, corresponding to the bonding distance of that pair of atoms. The peaks appearing in the PDF at distances larger than the nearest neighbors for each pair were flattened which means that the system no longer has regular arrangement at distances larger than the nearest neighbors, i.e. the structure can be considered amorphous. The PDF for Si-Si peaks around $3.1 \AA$ and for O-O peaks around $2.6 \AA$, as presented in Figs. 3.4 and 3.5. The nearest neighbor distances obtained in the present simulations are in good agreement with previous reports by Vashishta [36] and other authors using different potentials [58, 61]. In Fig. 3.6 the structural reorganization produced by the thermal treatment is illustrated; it can be seen that there is no a regular distribution of the particles in the amorphous system.

The angle distribution for the bonds $\mathrm{Si}-\mathrm{O}-\mathrm{Si}$ and $\mathrm{O}-\mathrm{Si}-\mathrm{O}$ for systems of amorphous silica at $300 \mathrm{~K}$ and $0 \mathrm{~K}$ are shown in the Fig. 3.7 and Fig. 3.8. Those distributions peak around $145^{\circ}$ for the $\mathrm{Si}-\mathrm{O}-\mathrm{Si}$ angle and around $109^{\circ}$ for the $\mathrm{O}-\mathrm{Si}-\mathrm{O}$ angle, values that are in good agreement with information collected using NMR and other computer simulations [36, 61. Note that the distributions at $0 \mathrm{~K}$ are as expected to be narrower than those at $300 \mathrm{~K}$.

\footnotetext{
${ }^{1}$ The energy unit used in Fig. 3.2 is the Hartree, $\mathrm{E}_{\mathrm{h}}$, which is equivalent to $4.360 \mathrm{x} 10^{-18} \mathrm{~J}$, or $27.211 \mathrm{eV}$, approximately.
} 


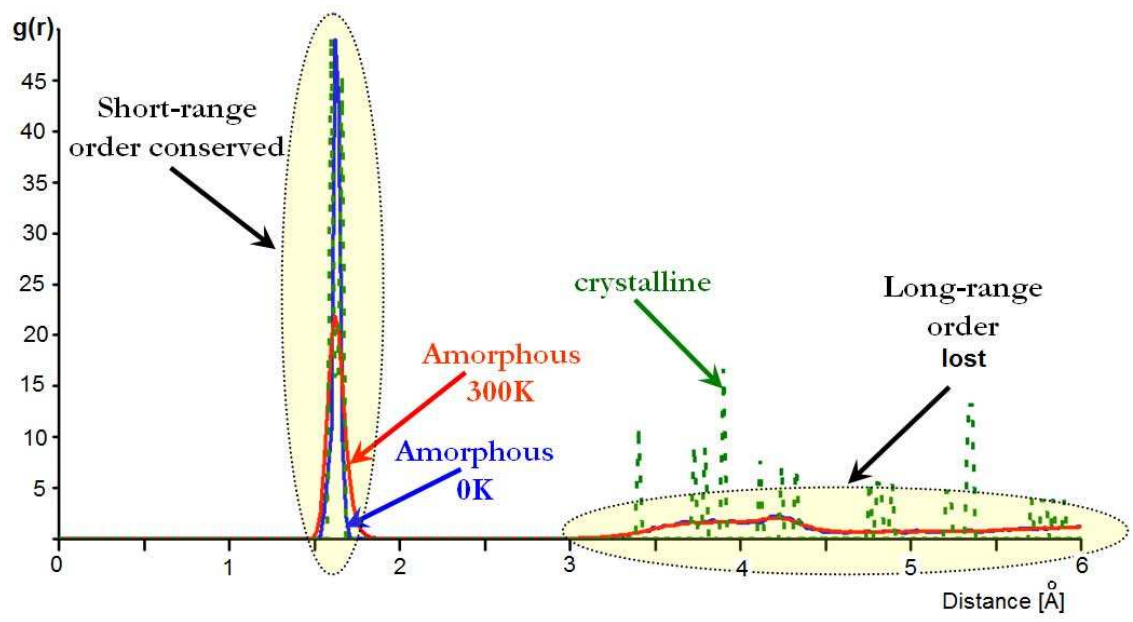

Figure 3.3: PDF of Si-O distances for $\beta$-cristobalite and amorphous silica at 300 and $0 \mathrm{~K}$

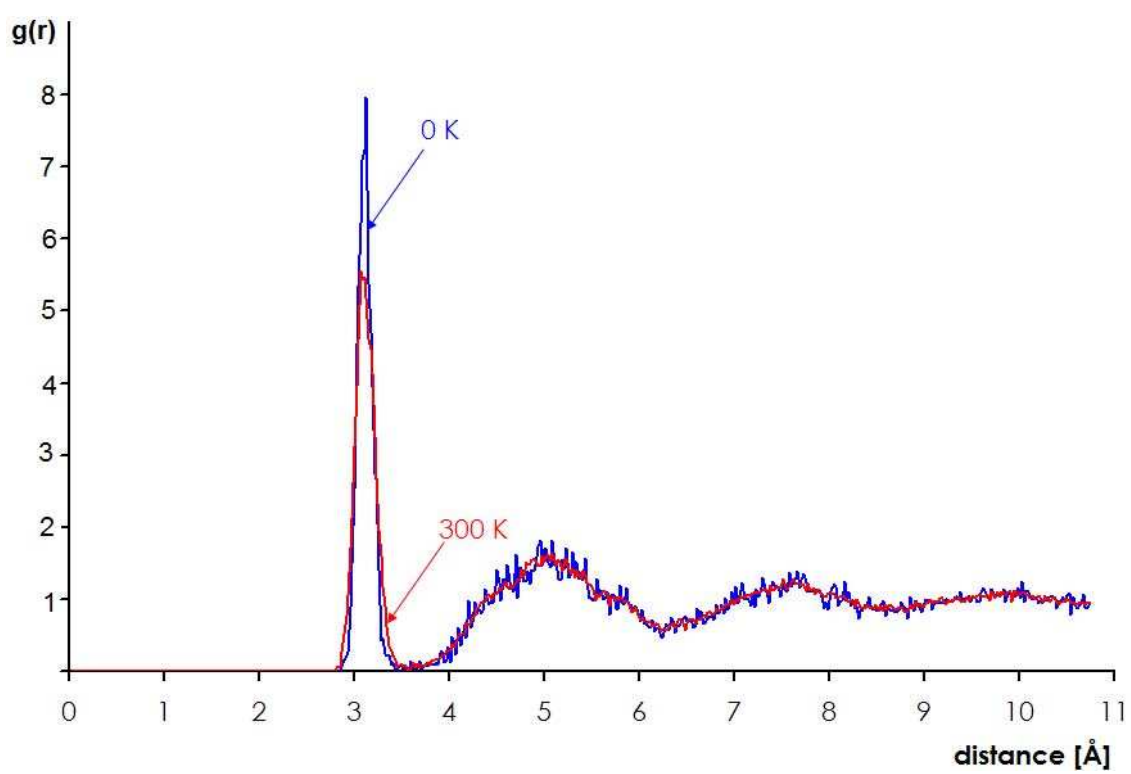

Figure 3.4: PDF of Si-Si distances for amorphous silica at $300 \mathrm{~K}$ and $0 \mathrm{~K}$ 


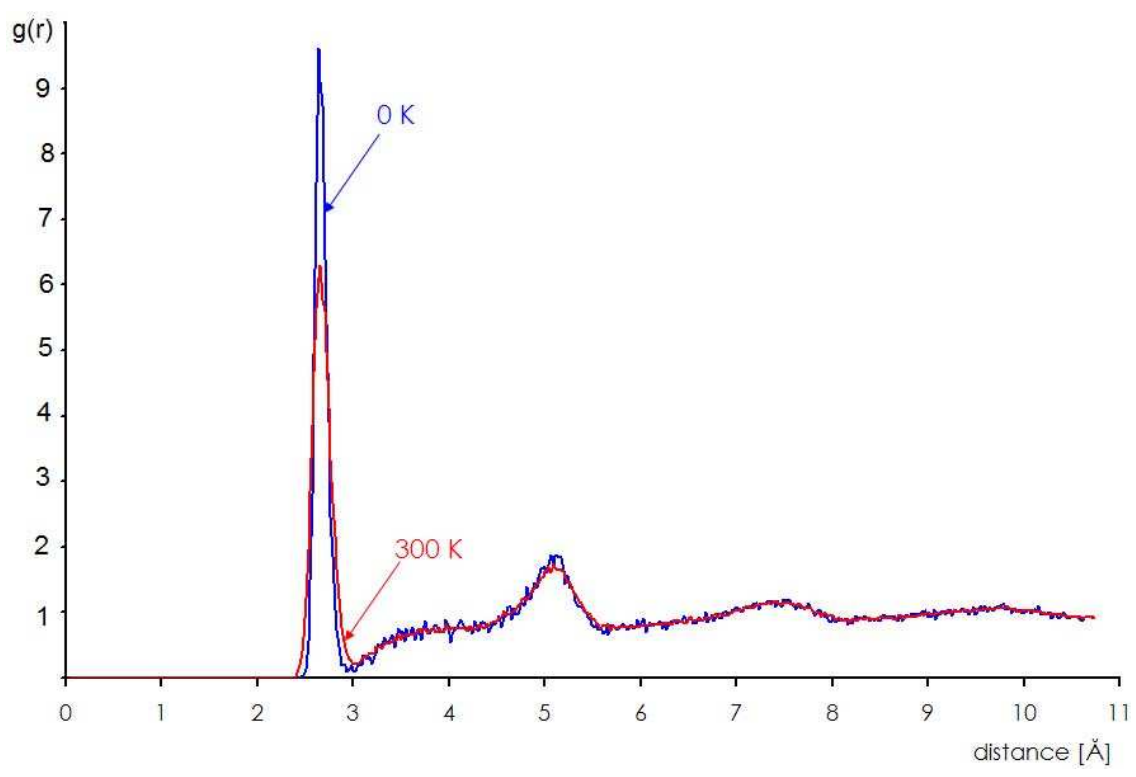

Figure 3.5: PDF of O-O distances for amorphous silica at $300 \mathrm{~K}$ and $0 \mathrm{~K}$
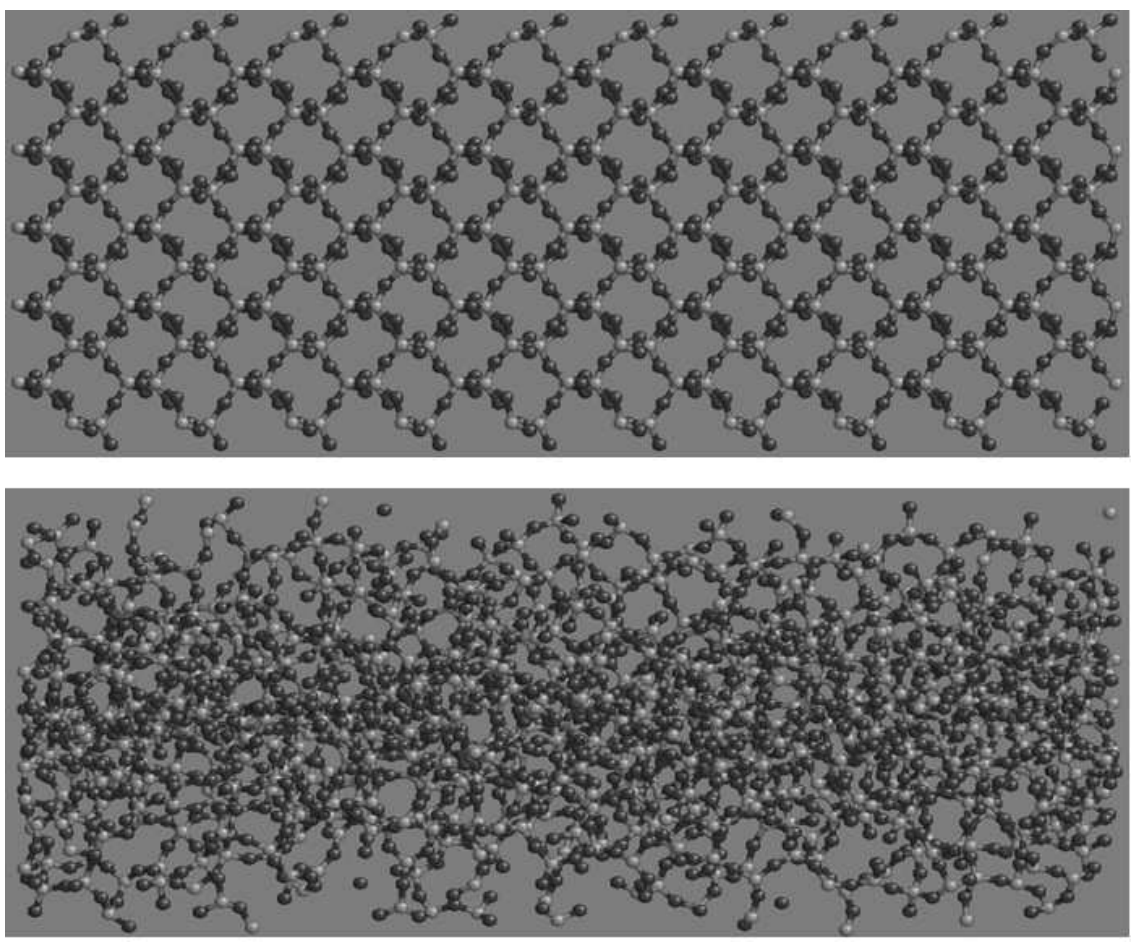

Figure 3.6: Snap-shoot of simulated silica samples, 2160 atoms, $71.7 \times 21.5 \times 21.5 \AA^{3}$. Top) $\beta$-cristobalite. Bottom) glass. Oxygen atoms in dark color, Silicon atoms in light color 62 


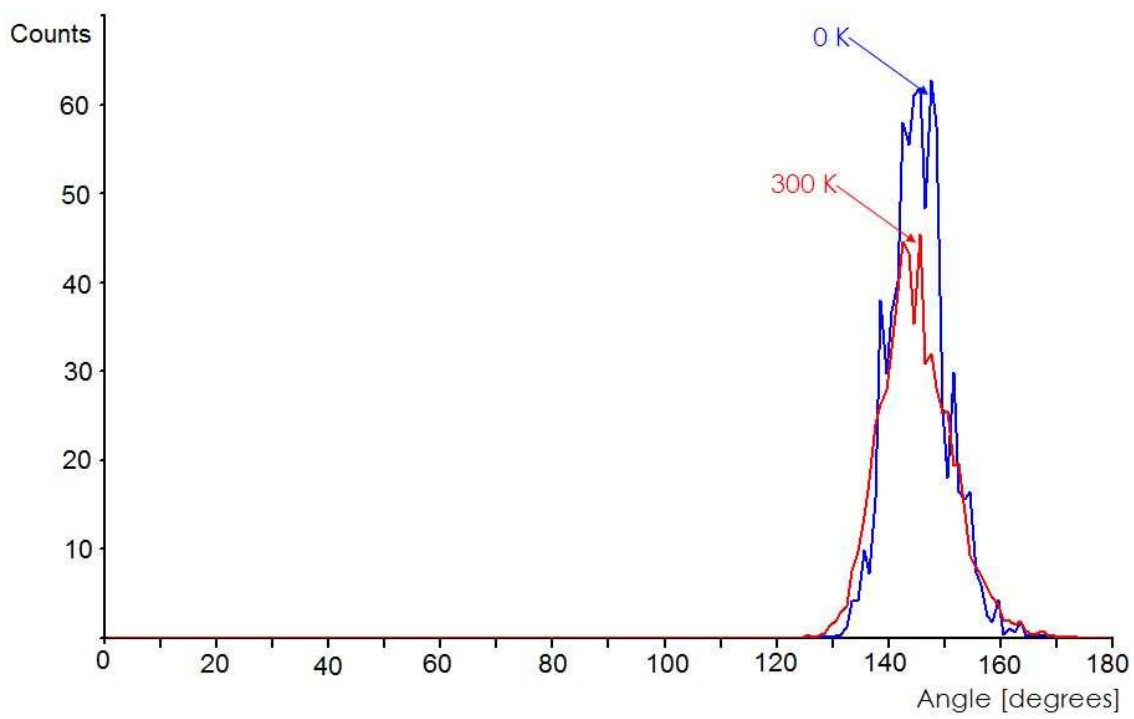

Figure 3.7: Si-O-Si angle distribution for amorphous silica at 300 and $0 \mathrm{~K}$

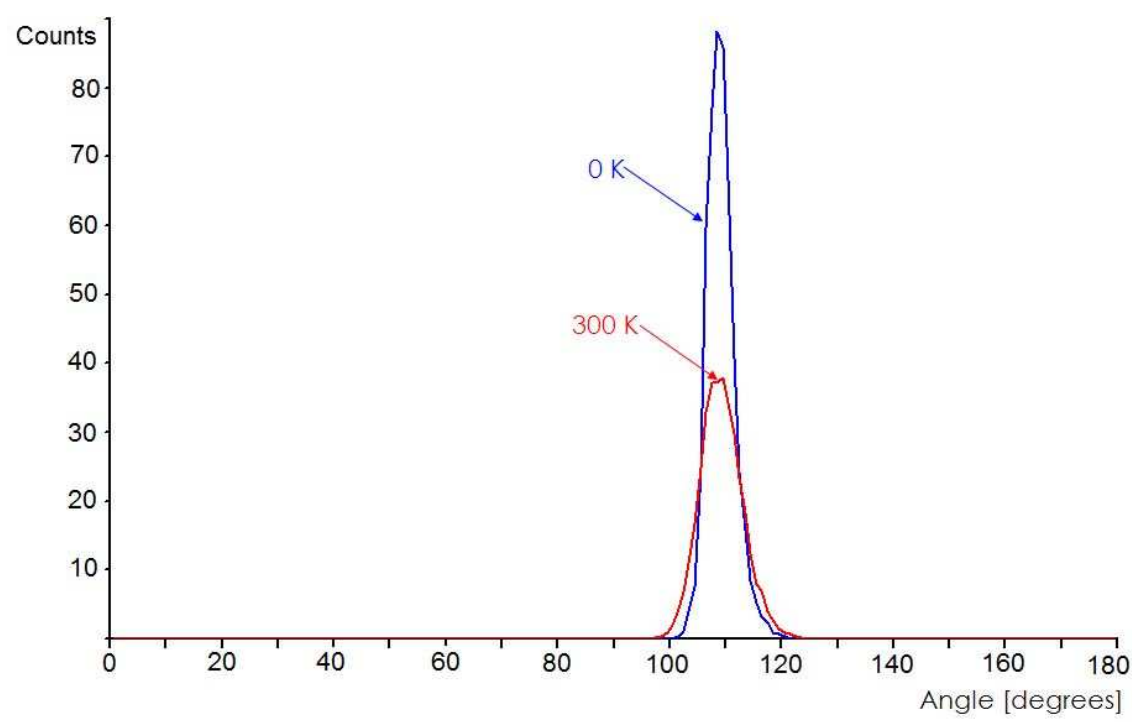

Figure 3.8: O-Si-O angle distribution for amorphous silica at 300 and $0 \mathrm{~K}$ 

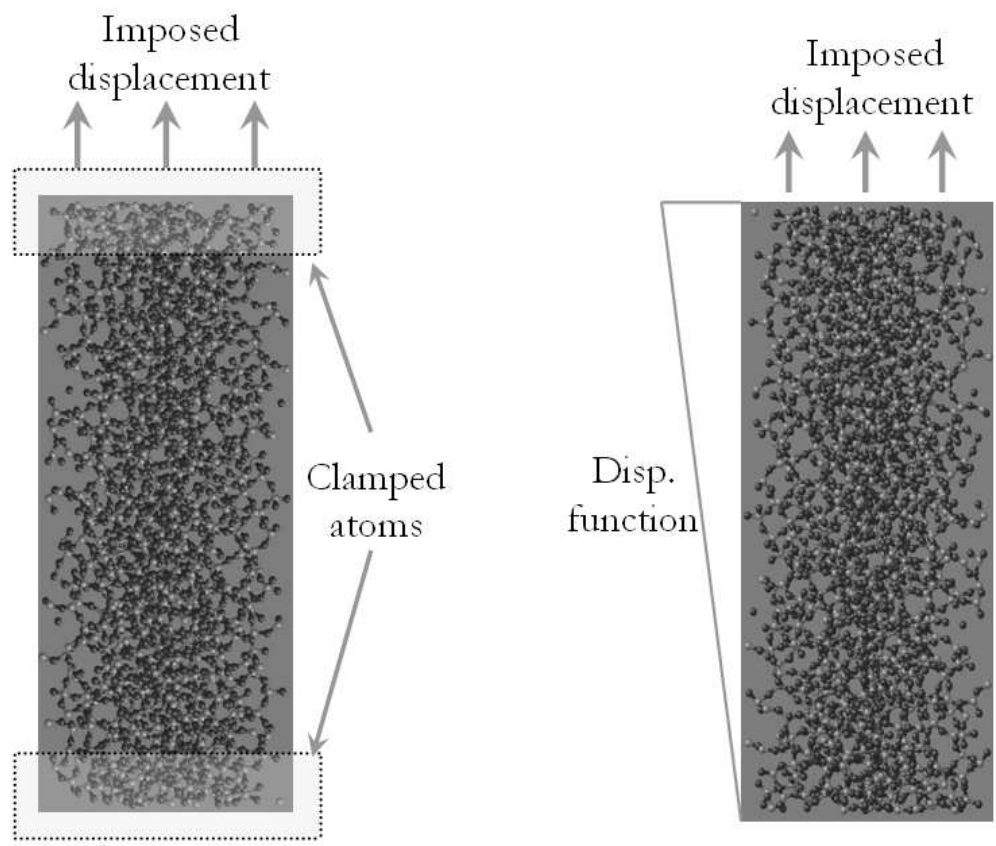

Fix atoms

Figure 3.9: Methods of stretching an atomic system. Left) Pulling. Right) Scaling

\subsubsection{Mechanical Characterization}

Modeling a tension tests of an atomic systems can be performed by clamping a group of atoms at both ends of the system and then pulling them apart, or keeping one end fixed and pulling the other. The atoms in the rest of the system will accommodate and reorganize to follow the perturbation introduced by the displacement of the edges. In this procedure the groups of atoms that have been clamped do not follow the same dynamics as the rest of the system, but this type of test is intuitively a good representation of laboratory experiments. Another way to model the stretching of the system consists of scaling all the atomic positions at the same time. This means that there is no delay in the propagation of the perturbation through the system, and since no clamping is needed, all atoms follow the same dynamics. A schematic representation of these two stretching procedures is presented in Fig. 3.9. In both cases, after the system has been stretched and relaxed the stresses can be calculated based on the interactomic forces and the dimensions of the sample using the approach discussed in section 2.2.3.

The elastic moduli of the samples is obtained from the stress and strain data at the beginning of the stretching process, as follows

$$
\begin{gathered}
\sigma_{x x}=E \varepsilon_{x x}+\nu\left(\sigma_{y y}+\sigma_{z z}\right)+\sigma_{x x}^{o} \\
\sigma_{y y}+\sigma_{z z}=\nu\left(2 \sigma_{x x}+\sigma_{y y}+\sigma_{z z}\right)+\left(\sigma_{y y}^{o}+\sigma_{z z}^{o}\right)
\end{gathered}
$$




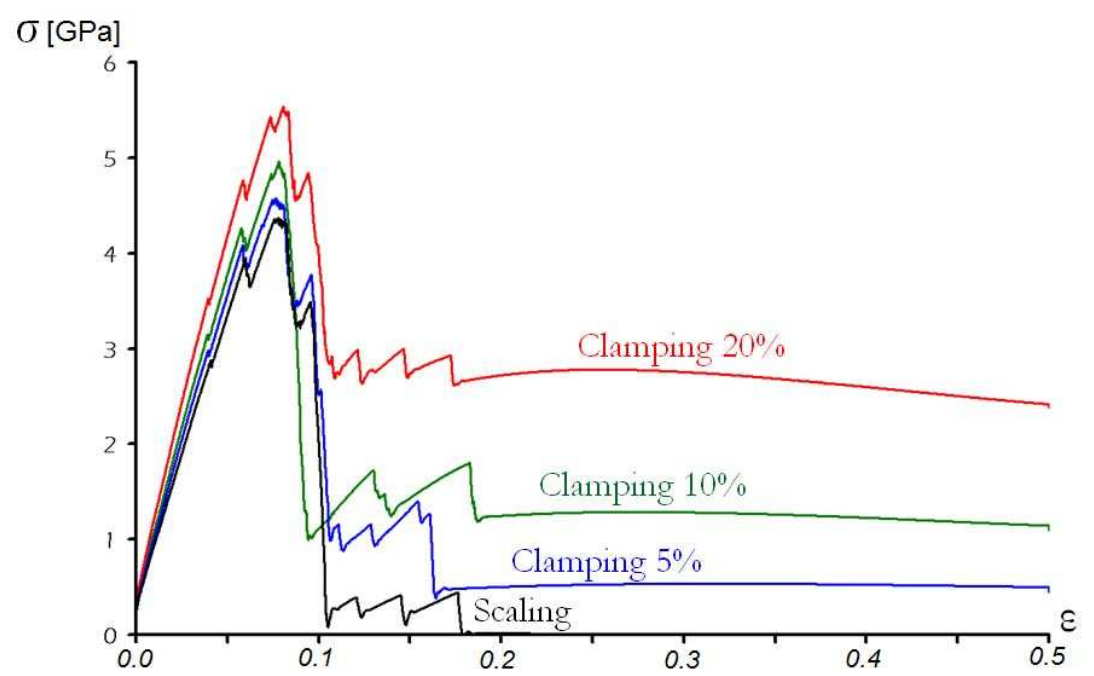

Figure 3.10: Stress-Strain plot for a silica glass sample stretched using a strain rate of $0.004 \mathrm{ps}^{-1}$. Comparison of pulling vs. scaling stretching procedures

Hooke's law as written in the Eq. 3.13 and Eq. 3.14 is applied to fit the data and determine the elastic modulus and the Poisson ratio. The strength is defined as the maximum value of stress recorded for the system. In those equations $\sigma_{x x}, \sigma_{y y}$, and $\sigma_{z z}$ represent the stress along the Cartesian axes. Although the systems are stretched along the x-direction only, $\sigma_{y y}$ and $\sigma_{z z}$ appear due to the periodic boundary conditions, because the system is not allowed to shrink or expand along y- and z-directions. The remaining quantities in Eq. 3.13 and Eq. 3.14 are the elastic modulus, E, the Poisson ratio, $\nu$, and $\sigma_{x x}^{o}, \sigma_{y y}^{o}$, and $\sigma_{z z}^{o}$ that represent the initial stresses in the system.

A comparison between tension tests performed by scaling and pulling, changing the size of the area clamped, is presented in the Fig. 3.10. Given that the group of atoms clamped for pulling do not follow the same dynamics than the rest of the system, pulling the sample generates fictitious stresses. This becomes clear after the sample has been broken in two separated pieces because there is no full relaxation of the stresses in the system. It seems obvious that if a bigger part of the system is clamped the fictitious stress is larger. The scaling of the system does a better representation of the results expected from a laboratory test because after the sample is completely broken the system is fully relaxed. Therefore, scaling the atomic position of the atoms was selected as the method to stretch the samples for all the subsequent tension tests.

The strain rate used for the simulations described above was $0.004 \mathrm{ps}^{-1}$, which is a large strain rate compared to those used in laboratory experiments. However, using high strain rates is necessary to produce results in reasonable computer time. The effect of the strain rate on the stress vs. strain behavior for the sample simulated in this study is presented in Fig. 3.11, It is observed that for high strain rates the strength of the sample is higher. However, after the sample has been broken in two separated pieces it is not fully relaxed. Using a strain rate of $0.004 \mathrm{ps}^{-1}$ it was 


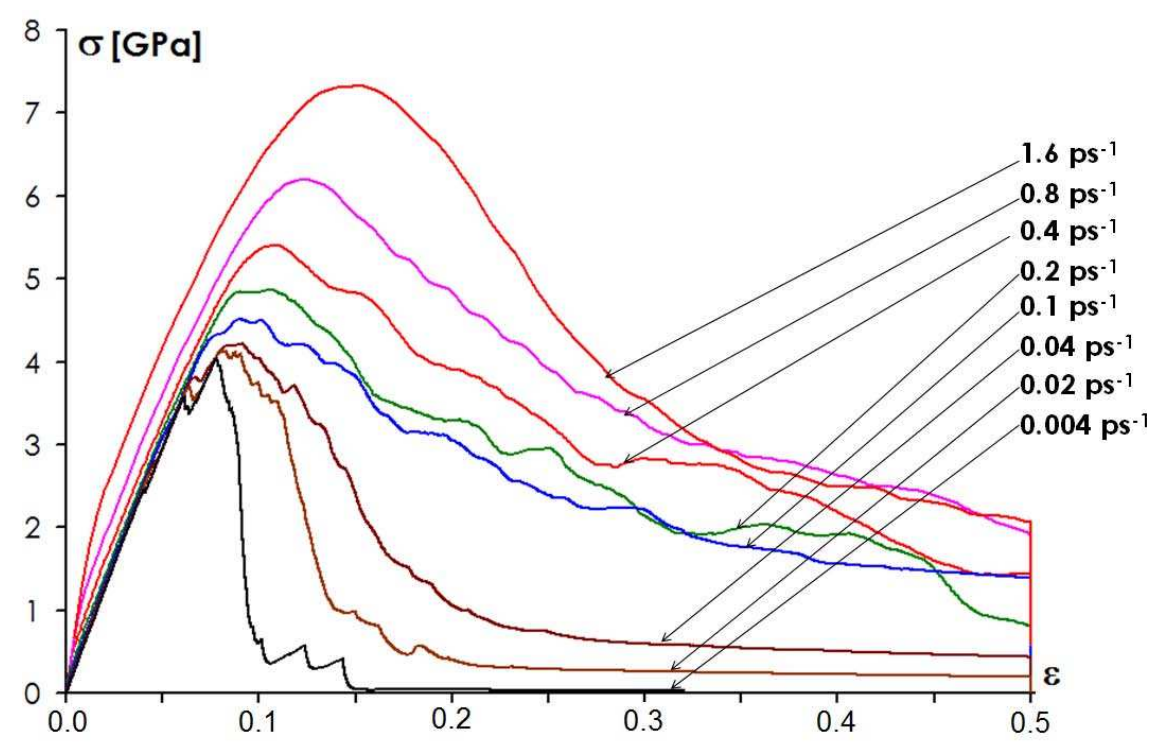

Figure 3.11: Stress-Strain plot for a silica glass sample stretched by scaling using different strain rates

observed that the stress relaxed after breaking the sample in two separated pieces. Therefore, this strain rate was selected for the subsequent tests.

Using a high strain rate during the simulation has the drawback that the atoms tend to speedup, which causes an unrealistic increase in the temperature of the atomic system. To model the experiment more realistically, it is necessary to implement a technique to control the speed of the atoms, thereby the temperature of the atomic system. A technique that has been proven successful in controlling the temperature during simulations of atomic systems consists of solving the dynamics of the systems not in the form of Newton's second law but in the form of a Langevin equation 63

$$
m_{i} \ddot{\vec{r}}_{i}-m_{i} \gamma \dot{\vec{r}}_{i}=\vec{F}_{i}+\vec{R}_{i}
$$

where $m_{i}, \ddot{\vec{r}}_{i}$, and $\dot{\vec{r}}_{i}$ are the mass, the acceleration and the velocity of atom $i$ respectively, $\vec{F}_{i}$ represents the interatomic force exerted by all the atoms in the system on atom $i, \overrightarrow{R_{i}}$ is a stochastic force related to the current and targeted temperatures of the system, and $\gamma$ is a damping factor. Using the Langevin equation to represent the dynamics of the system produces a gradual scaling of the speed of the atoms in order to match the desired temperature of the system when a temperaturecontrolled ensemble is modeled.

The damping coefficient has a strong influence on the results of the simulation, therefore it must be selected according to the system that is being modeled. The effect of the damping coefficient is presented in the Fig. 3.12, a stress vs. strain plot for a silica sample stretched by scaling at a strain rate of $0.004 \mathrm{ps}^{-1}$. In Fig. [3.12, low damping means that the speed of atoms is gradually modified to adjust the temperature of the system, while high damping means that the speed is 


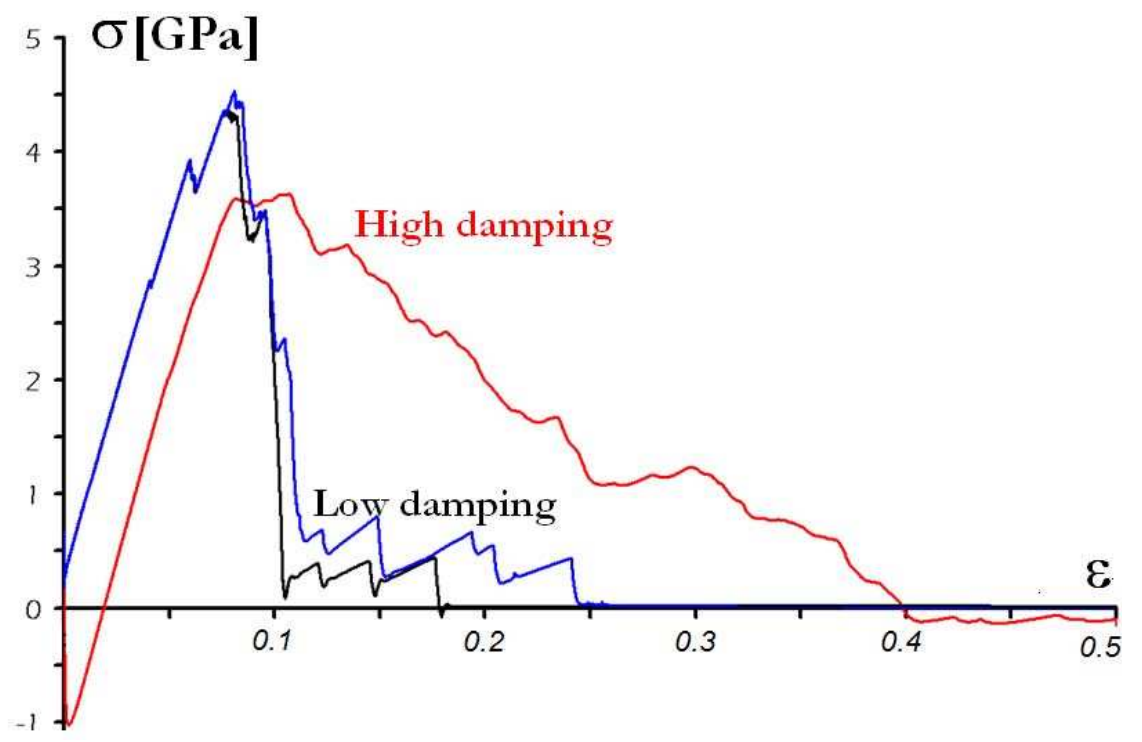

Figure 3.12: Stress-Strain plot for a silica glass sample stretched by scaling at a strain rate of $0.004 \mathrm{ps}^{-1}$. Effect of the damping coefficient in the Langevin equation

modified rather fast (in a few time steps). According to what is seen in Fig. 3.12 the damping to be selected for the simulations was low $\left(0.001 \mathrm{fs}^{-1}\right)$.

Additional information about the selection of the damping factor can be obtained by comparing simulations performed with and without the temperature control scheme. In Fig. 3.13 the stress vs. strain plot for one of such comparisons is shown. The initial temperature of the samples was set at $0 \mathrm{~K}$. Removing the temperature control in the system causes the stress to fluctuate, specially after the sample starts breaking. Once the sample starts breaking the atoms in the vicinity of the breaking regions develop large acceleration and speed increasing the temperature of the system and relaxing the stress. That is the reason why the system without temperature control breaks at a stress level slightly smaller than the temperature-controlled system. Additionally, for the system without temperature control, after the sample is broken in two pieces, the parts are at higher temperature than they were at the early stages of the simulation which causes fluctuations in the stress. The parameters of the controlled temperature simulation must allow keeping the system under isothermal conditions without disrupting the dynamics of the atoms.

The final test performed on the dense silica systems was used to investigate the effect of the system size on the simulation results. In Fig. 3.14 the stress vs. strain plot corresponding to samples stretched by scaling at a strain rate of $0.004 \mathrm{ps}^{-1}$ is shown. The elastic modulus and the strength for the three systems are presented in Table 3.2. This test showed that there is no change in the properties by increasing the size of the system. This is largely due to the use of periodic boundary conditions which make the modeled system virtually of infinite volume. It is important to notice that the elastic modulus obtained here deviates only by about $12 \%$ from the experimental 


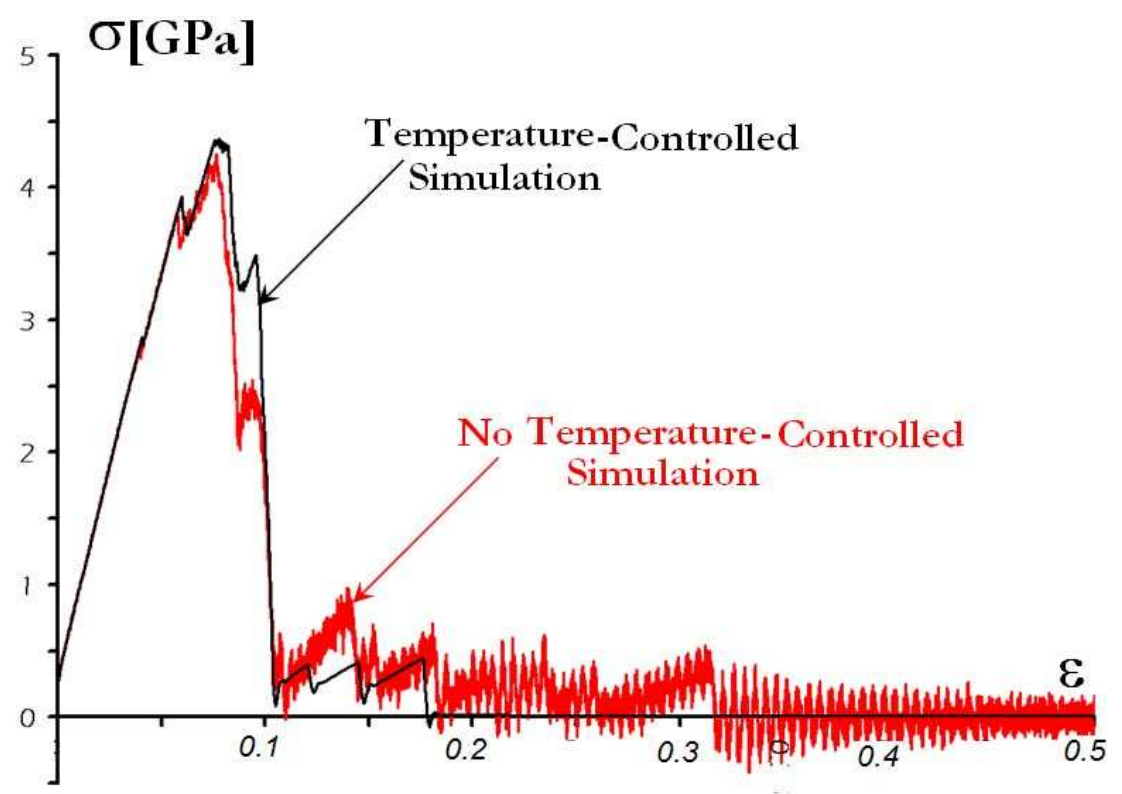

Figure 3.13: Stress-Strain plot for a silica glass sample stretched by scaling at a strain rate of $0.004 \mathrm{ps}^{-1}$. Comparison of simulations with and without temperature control

\begin{tabular}{ccc}
\hline System size $\left[\AA^{3}\right]$ & $\mathbf{E}[\mathbf{G P a}]$ & $\sigma[\mathbf{G P a}]$ \\
\hline $71 \times 21 \times 21$ & 60 & 4.4 \\
\hline $71 \times 71 \times 71$ & 63 & 4.4 \\
\hline $142 \times 142 \times 142$ & 63 & 4.3
\end{tabular}

Table 3.2: Elastic modulus, E, and strength, $\sigma$, for dense silica modeled with different system sizes

value. However, increasing the transverse section from $21 \times 21 \AA^{2}$ to $71 \times 71 \AA^{2}$ and to $142 \times 142$ $\AA^{2}$ increases the strain that the system can sustain before breaking apart. The reason for this is that when a small transverse section is used each bond forming that section represents a greater percentage of it compared to when a bigger section is used. For example, if the transverse section is formed by four bonds each would represent $25 \%$ of the section and using a transverse section formed by 8 bonds each one represents only $12.5 \%$ of the section.

The conclusions obtained from modeling dense silica are applied to modeling porous silica (aerogels and xerogels), which is discussed in the next section.

\subsection{Model of Silica Aerogels and Xerogels}

This section contains an extended version of the work published by the author in the Journal of Non-Crystalline Solids 2010 [64]. In that study the structural and mechanical properties of computational samples of silica aerogels and xerogels characterized at $0 \mathrm{~K}$ were presented. The procedure to prepare the compuational porous samples consisted in expanding single crystals of $\beta$ - 


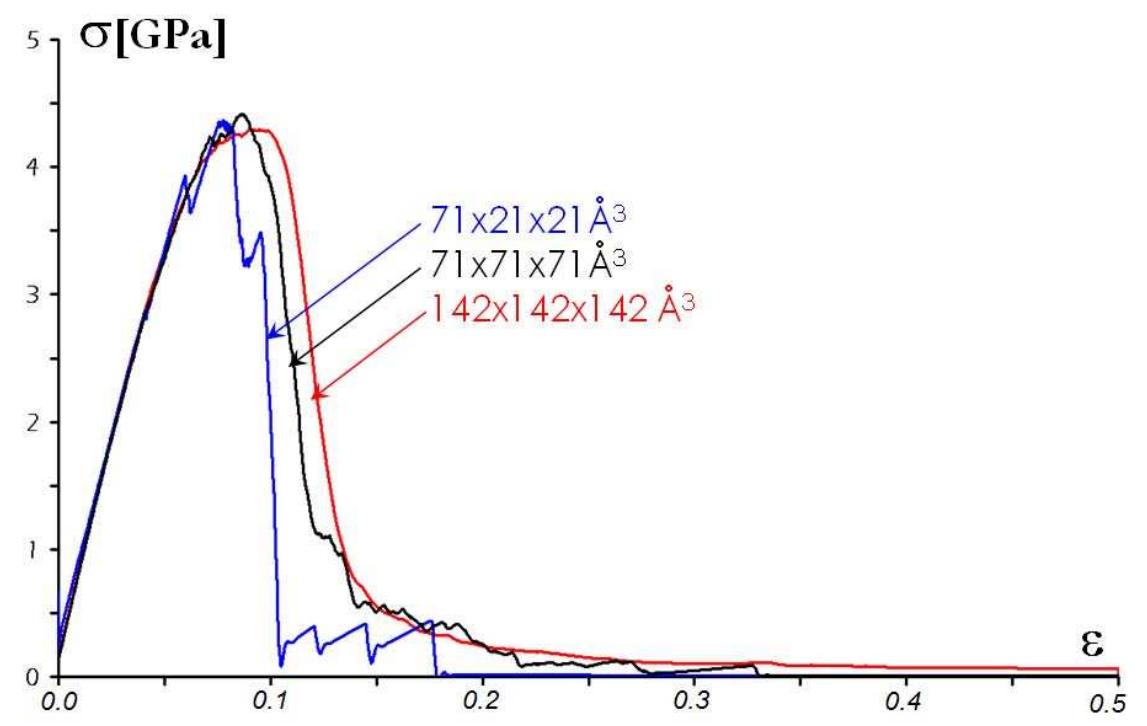

Figure 3.14: Stress-Strain plot for a silica glass sample stretched by scaling at a strain rate of $0.004 \mathrm{ps}^{-1}$. Effect of the size of the system

cristobalite to reach the desired density followed by a thermal treatment. The resulting structures have densities in the range from 0.23 to $2.2 \frac{\mathrm{g}}{\mathrm{cm}^{3}}$. The structures are studied by obtaining the fractal dimension using the methods described in chapter 2, The fractal dimension found was in good agreement with previously published data from experiments and comparable computer simulations. The mechanical properties, namely elastic modulus and strength, are studied through the simulation of a tension test. The elastic modulus and the strength relate to density by a power law characterized with exponents of $3.11 \pm 0.21$ and $2.53 \pm 0.15$, respectively. These values compare well with previously published data. The results of this study proved that the direct expansion of a compuataional crystalline sample, coupled with thermal processing, leads to porous systems suitable to investigate the structure and the mechanical properties of silica aerogels.

\subsubsection{Sample Preparation}

The procedure to create the computational samples follow these steps:

i Placing the atoms at the crystalline positions of $\beta$-cristobalite with a lattice constant corresponding to the desired density. For instance, if the samples were to be dense silica, as in the previous section, the lattice constant would have been $7.13 \AA$.

ii Heating up the system up to $3,000 \mathrm{~K}$ and cooling it down allowing relaxation at different temperatures, following the same procedure described in the previous section for dense silica samples.

The crystalline samples used have a size of approximately $71^{3} \AA^{3}$ containing 24,000 atoms. They are expanded to obtain eight different systems with volumes of $81^{3}, 91^{3}, 101^{3}, 111^{3}, 121^{3}$, 
$131^{3}, 141^{3}$, and $151^{3} \AA^{3}$, and having densities of about $1.49,1.05,0.77,0.58,0.45,0.35,0.28$, and $0.23 \frac{\mathrm{g}}{\mathrm{cm}^{3}}$, respectively. The simulations were performed using periodic boundary conditions along the three Cartesian direction and using a time step of $0.5 \mathrm{fs}$. The selection of a small time step ensures an accurate tracking of the trajectory of the atoms, which is important in this case because large free surfaces are generated inside the samples.

In Fig. 3.15 2D projections of the structures generated for this study are shown. In the images it can be seen that when the density is reduced clusters of atoms interconnected by small branches are formed in the system. The same structure is found in aerogel samples studied using high resolution TEM [1].

The preparation procedure described above is not intended to represent the actual low temperature sol-gel process that is used to produce physical samples of aerogels and xerogels. The procedure implemented here has for goal to generate computational samples with a structure comparable to that of physical aerogels and xerogels. The computational samples created are found suitable to investigate some of the structural and mechanical properties silica aerogels and xerogels.

\subsubsection{Structural Characterization}

The Pair Distribution Functions (PDF) of the porous samples are shown in Fig. 3.16, along with that of a dense silica glass for comparison. From the figures it can be concluded that the short-range order of the structures is preserved at the characterization temperature, which was set at $0 \mathrm{~K}$. The bonding distance between $\mathrm{Si}$ and $\mathrm{O}$ atoms spreads from $1.4 \AA$ to $1.8 \AA$ for all the samples, the maximum is found at $1.6 \AA$. The intermediate range order (distances from $5 \AA$ to $20 \AA$ ) changes as the density of the samples is reduced. As it is illustrated in the Fig. 3.17, for the dense sample the peaks of the PDF cross the line $g=1$, and for distances greater than $10 \AA$ the PDF tends to 1 . As the density of the sample is reduced the peaks of the PDF in the intermediate range region grow above the line $g=1$ and the function gradually approaches 1 for distances greater than $10 \AA$. As mentioned in chapter 2, that gradual decay of the PDF is associated with the fractal dimension of the samples [41].

The distribution of the bonding angles $\mathrm{O}-\mathrm{Si}-\mathrm{O}$ and $\mathrm{Si}-\mathrm{O}-\mathrm{Si}$ is another way to analyze the changes caused for the reduction of the density of the samples. In Figs. 3.18 and 3.19 those distributions are shown for all the samples used in this study. The Si-O-Si angle distribution has its major peak around $144^{\circ}$ and the O-Si-O angle distribution has it around $109^{\circ}$; both values are in good agreement with results from dense silica samples. In Figs. 3.18 and 3.19 it is shown that, as the density of the sample is reduced, a small peak appears next to the main peak of the distribution. For the O-Si-O angle distribution the small peak appears around $120^{\circ}$, and for the Si-O-Si angle distribution it does around $135^{\circ}$. Those peaks are related to the formation of free surfaces inside the structure. 


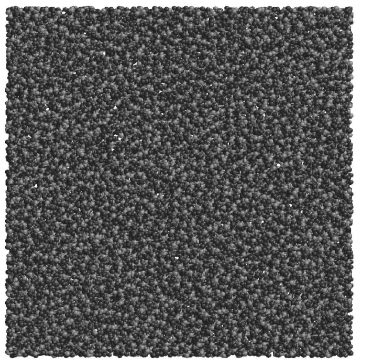

(a)

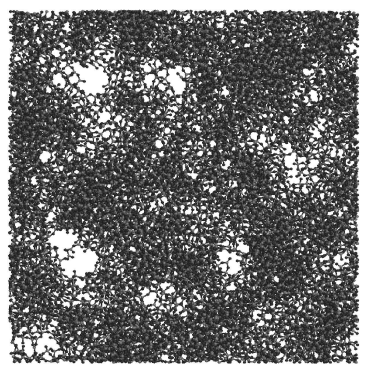

(d)

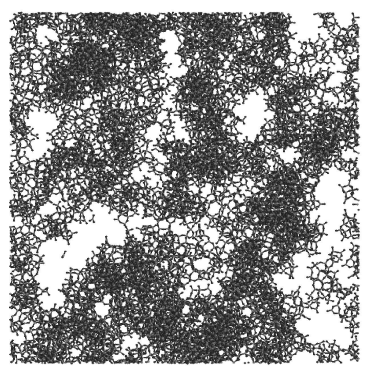

(g)

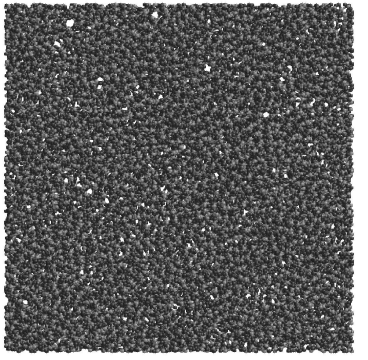

(b)

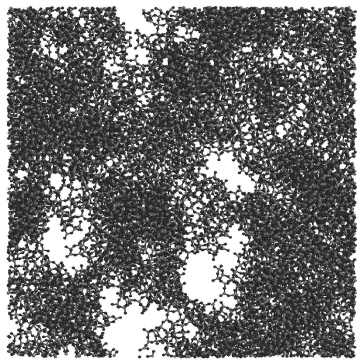

(e)

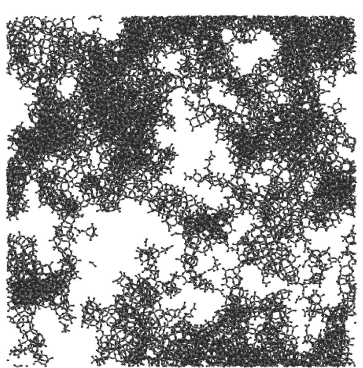

(h)

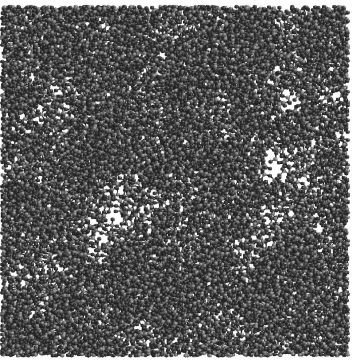

(c)

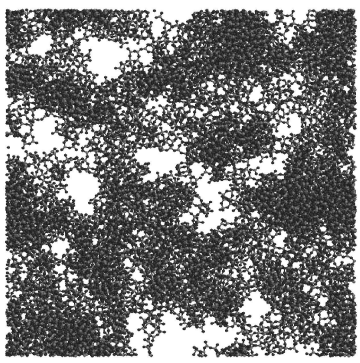

(f)

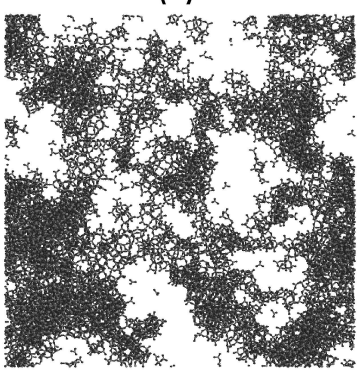

(i)

Figure 3.15: Samples generated for the study of the properties of silica aerogels and xerogels 62. (a) $\rho=2.2$ $\frac{\mathrm{g}}{\mathrm{cm}^{3}}$, (b) $\rho=1.49 \frac{\mathrm{g}}{\mathrm{cm}^{3}}$, (c) $\rho=1.05 \frac{\mathrm{g}}{\mathrm{cm}^{3}}$, (d) $\rho=0.77 \frac{\mathrm{g}}{\mathrm{cm}^{3}}$, (e) $\rho=0.58 \frac{\mathrm{g}}{\mathrm{cm}^{3}}$, (f) $\rho=0.45 \frac{\mathrm{g}}{\mathrm{cm}^{3}}$, (g) $\rho=0.35 \frac{\mathrm{g}}{\mathrm{cm}^{3}}$, (h) $\rho=0.28 \frac{\mathrm{g}}{\mathrm{cm}^{3}}$, (i) $\rho=0.23 \frac{\mathrm{g}}{\mathrm{cm}^{3}}$ 

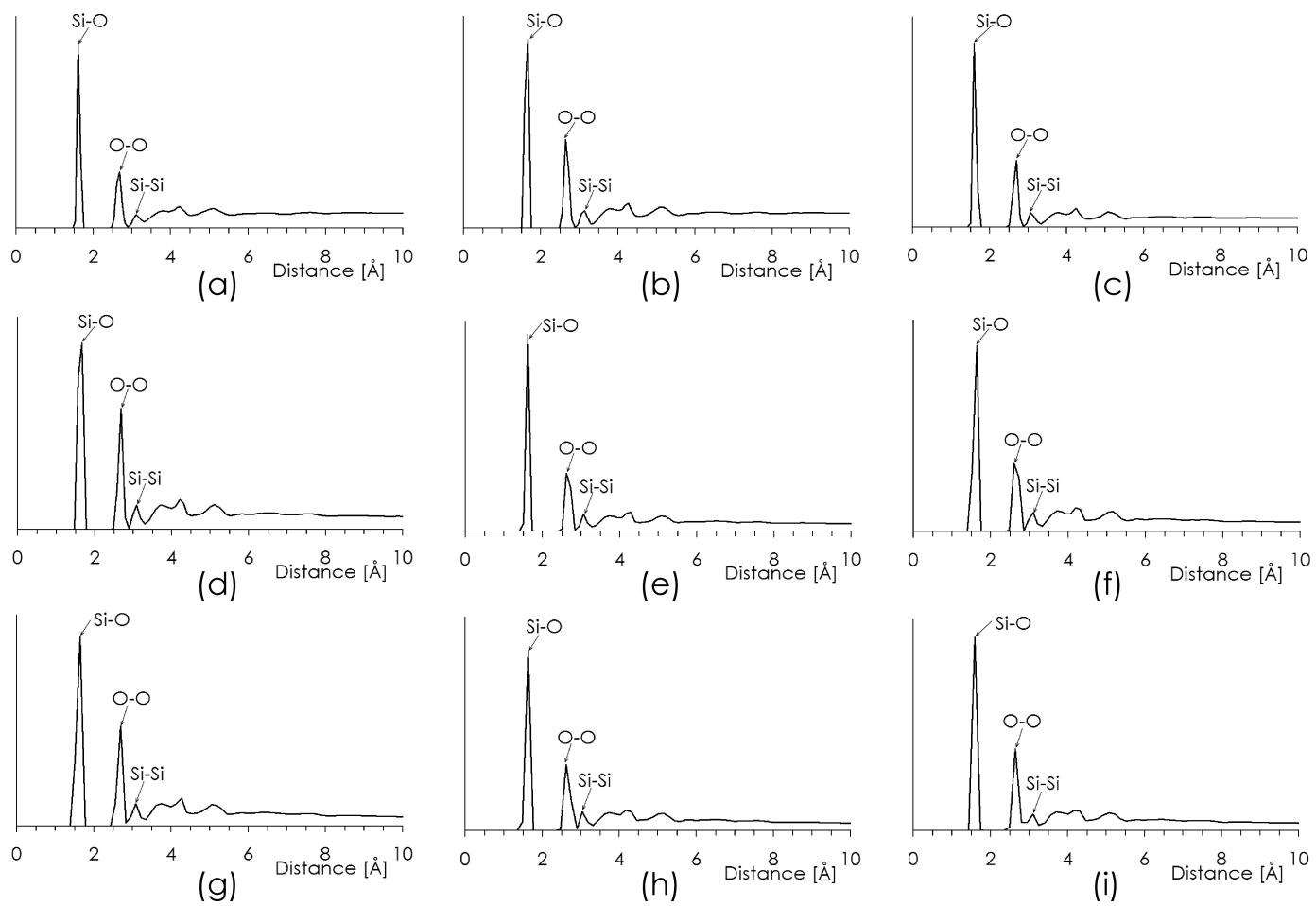

Figure 3.16: PDF for samples generated for the study of the properties of silica aerogels and xerogels. (a) $\rho=2.2 \frac{\mathrm{g}}{\mathrm{cm}^{3}}$, (b) $\rho=1.49 \frac{\mathrm{g}}{\mathrm{cm}^{3}}$, (c) $\rho=1.05 \frac{\mathrm{g}}{\mathrm{cm}^{3}}$, (d) $\rho=0.77 \frac{\mathrm{g}}{\mathrm{cm}^{3}}$, (e) $\rho=0.58 \frac{\mathrm{g}}{\mathrm{cm}^{3}}$, (f) $\rho=0.45 \frac{\mathrm{g}}{\mathrm{cm}^{3}}$, (g) $\rho=0.35$ $\frac{\mathrm{g}}{\mathrm{cm}^{3}}$, (h) $\rho=0.28 \frac{\mathrm{g}}{\mathrm{cm}^{3}}$, (i) $\rho=0.23 \frac{\mathrm{g}}{\mathrm{cm}^{3}}$

Using the decay of the PDF in the intermediate range order, illustrated in the Fig. 3.17, the fractal dimension for all the samples is calculated according to Eq. 2.37. Additionally, the fractal dimension is obtained from simulated scattering experiments, which are based on the application of the Eq. 2.38. The scattering intensity vs. wave number plots are shown in Fig. 3.20, the slope of the linear part of the plots corresponds to the fractal dimension of the samples.

The values of the fractal dimension of the samples obtained from the PDF and through the simulated scattering experiments are presented in Fig. 3.21, along with the values of samples simulated by two different procedures and experimental measurements done by SANS. The simulated results produced here compare well with those produced by other methods. Although the procedure used to generate the porous samples for this study is not an exact representation of the events occurring during the polymerization and gelation of a silica solution, it produces samples having geometrical features which are very similar to those of real aerogels and xerogels. The fractal dimension is slightly smaller than the experimental results but it relates to the density in a similar way as the experimental results. This leads to the conclusion that the direct expansion of crystalline samples, as implemented here, allows the formation of fractal structures. 

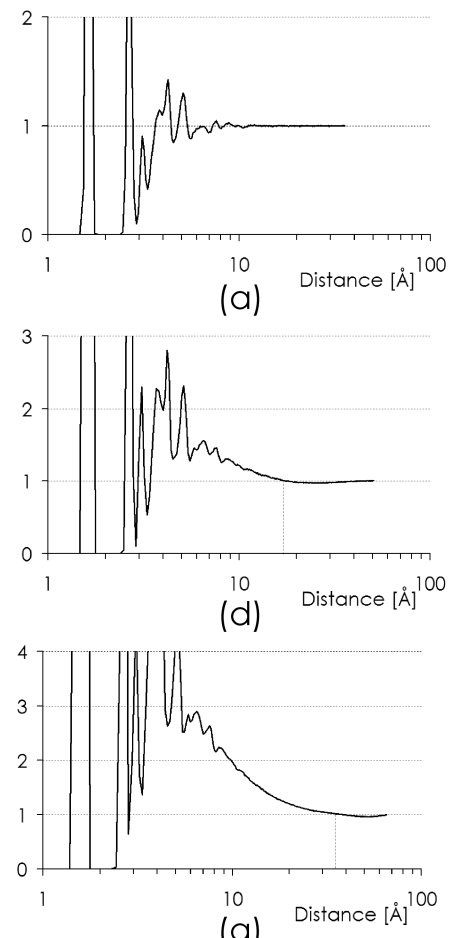

(g)

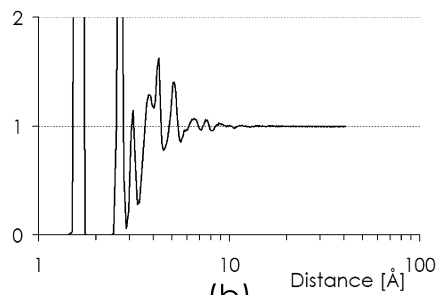

(b)
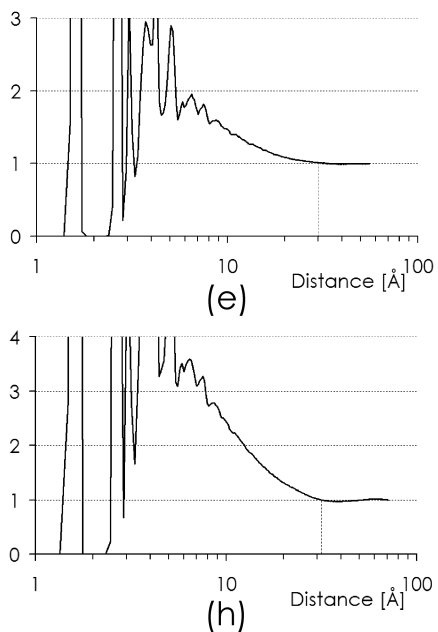

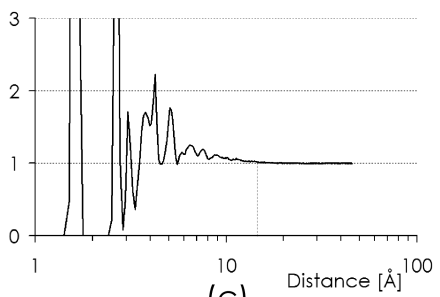

(c)

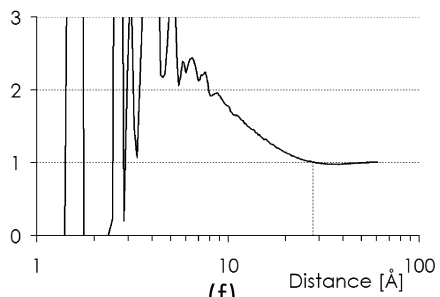

(f)

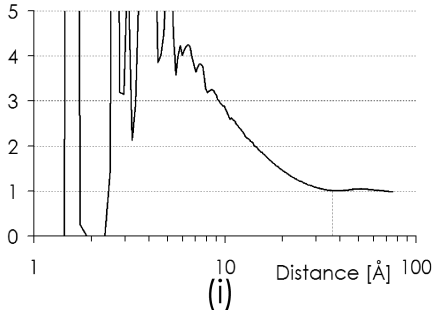

Figure 3.17: PDF in the intermediate range for samples generated for the study of the properties of silica aerogels and xerogels. (a) $\rho=2.2 \frac{\mathrm{g}}{\mathrm{cm}^{3}}$, (b) $\rho=1.49 \frac{\mathrm{g}}{\mathrm{cm}^{3}}$, (c) $\rho=1.05 \frac{\mathrm{g}}{\mathrm{cm}^{3}}$, (d) $\rho=0.77 \frac{\mathrm{g}}{\mathrm{cm}^{3}}$, (e) $\rho=0.58 \frac{\mathrm{g}}{\mathrm{cm}^{3}}$, (f) $\rho=0.45 \frac{\mathrm{g}}{\mathrm{cm}^{3}}$, (g) $\rho=0.35 \frac{\mathrm{g}}{\mathrm{cm}^{3}}$, (h) $\rho=0.28 \frac{\mathrm{g}}{\mathrm{cm}^{3}}$, (i) $\rho=0.23 \frac{\mathrm{g}}{\mathrm{cm}^{3}}$

\subsubsection{Mechanical Characterization}

Tension tests were simulated for all the samples generated. The conditions for the simulated tests were similar to those used for dense silica samples described in the previous section. A strain rate of $0.004 \mathrm{ps}^{-1}$ was applied by scaling the atomic positions of the atoms until the strain was equal to 0.5. Langevin dynamics with a damping factor of $1 \mathrm{ps}^{-1}$ was used to keep the temperature of the system at $0 \mathrm{~K}$. In Fig. 3.23 a $2 \mathrm{D}$ projection of a silica sample of density $0.45 \frac{\mathrm{g}}{\mathrm{cm}^{3}}$ is shown before and after the tension test. Additional figures depicting 2D projections of the initial and final states of the other samples used for this study are included in appendix A. The stress-strain relationships for all the samples are presented in the Fig. 3.22 ,

Before the tension test, the samples have residual stresses due to the generation process. However, the elastic region of the stress vs. strain curve for almost all the samples is highly linear, therefore accuracy of the elastic modulus is not expected to be affected. Additionally, for the samples of lower density after the strain has reached 0.5 the stress has not relaxed to zero. Although the samples can not carry more load, they have not been broken in two separated pieces. The observation becomes clear after looking at Fig. A.2 where it can be seen that at the end of the test small chains of atoms are connecting larger clusters. Fig. 3.22(i) is particularly interesting because the sample is so diluted inside the simulation box that there is always an interaction, although 

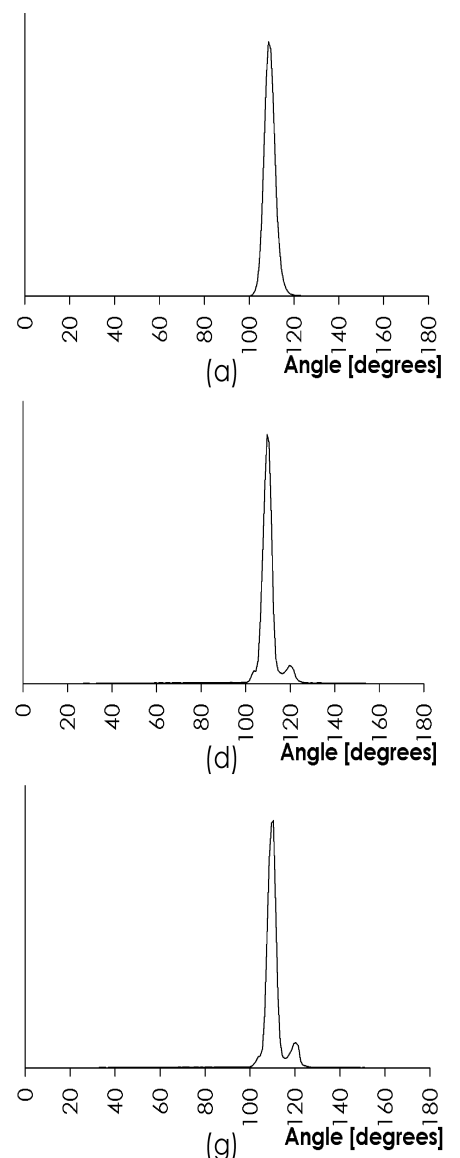

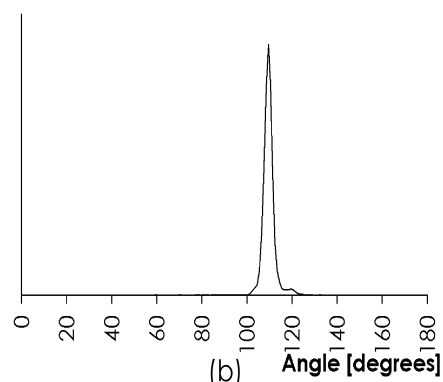

(b) Ānglē [dégrees]
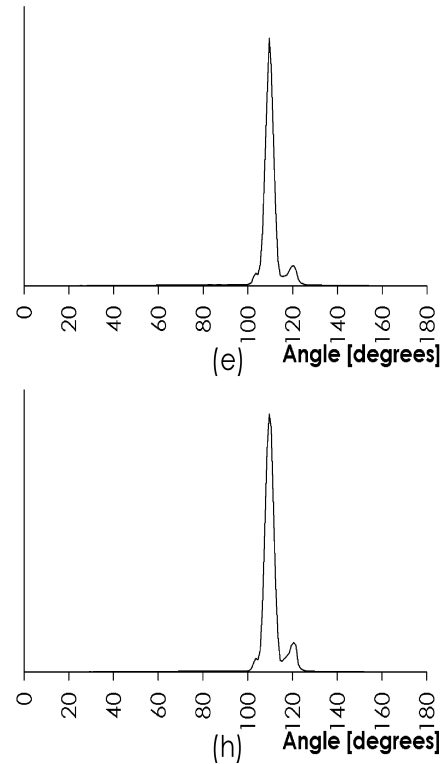
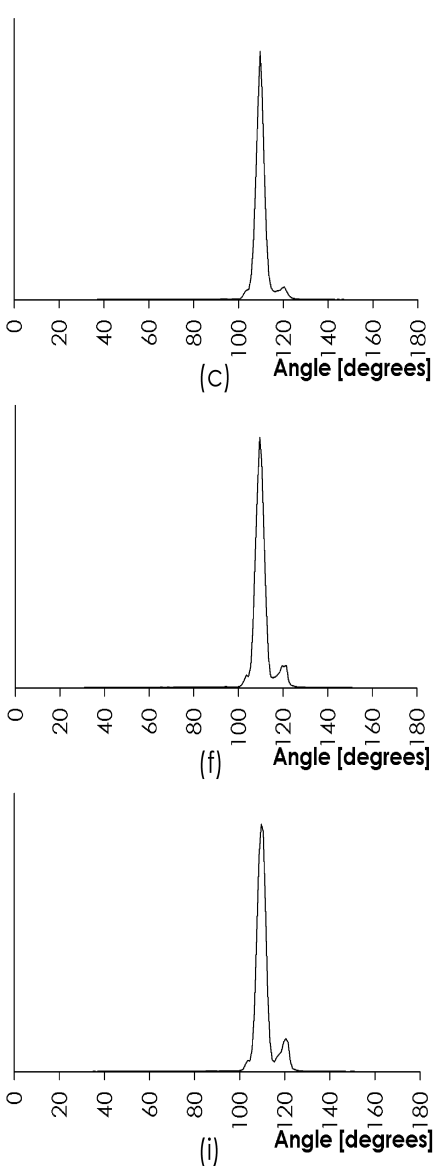

Figure 3.18: O-Si-O angel distribution for samples generated for the study of the properties of silica aerogels and xerogels. (a) $\rho=2.2 \frac{\mathrm{g}}{\mathrm{cm}^{3}}$, (b) $\rho=1.49 \frac{\mathrm{g}}{\mathrm{cm}^{3}}$, (c) $\rho=1.05 \frac{\mathrm{g}}{\mathrm{cm}^{3}}$, (d) $\rho=0.77 \frac{\mathrm{g}}{\mathrm{cm}^{3}}$, (e) $\rho=0.58 \frac{\mathrm{g}}{\mathrm{cm}^{3}}$, (f) $\rho=0.45$ $\frac{\mathrm{g}}{\mathrm{cm}^{3}}$, (g) $\rho=0.35 \frac{\mathrm{g}}{\mathrm{cm}^{3}}$, (h) $\rho=0.28 \frac{\mathrm{g}}{\mathrm{cm}^{3}}$, (i) $\rho=0.23 \frac{\mathrm{g}}{\mathrm{cm}^{3}}$ 


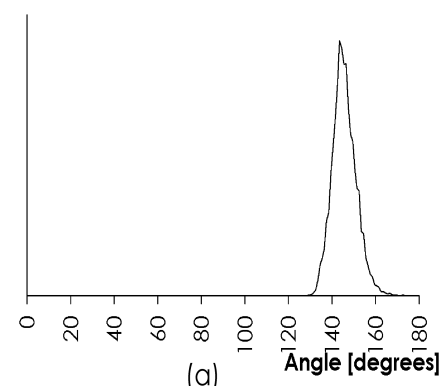

(a)
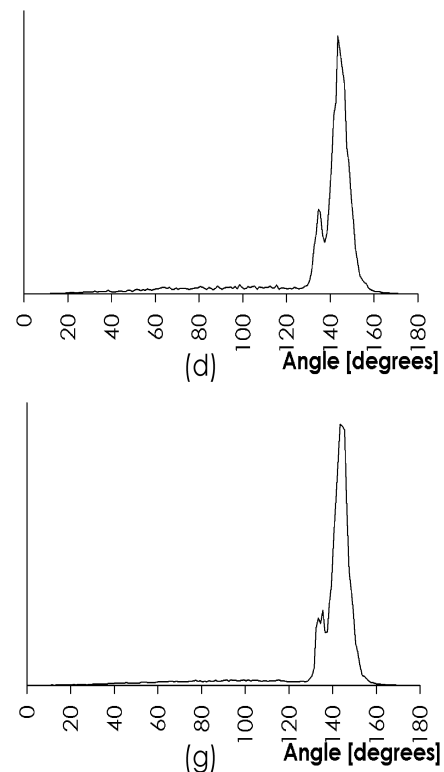

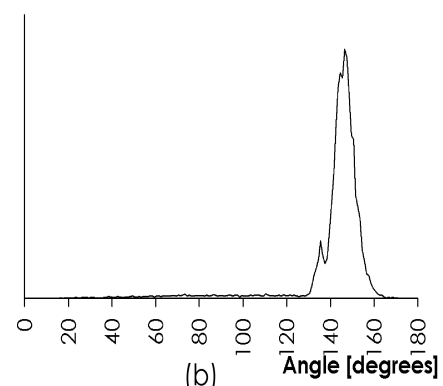

(b)

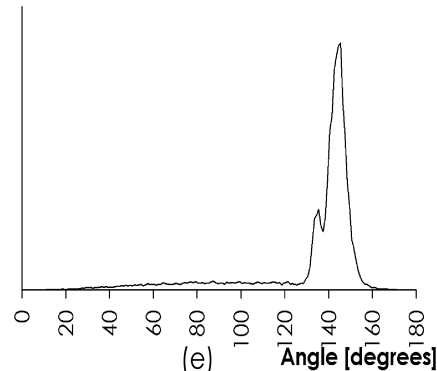

(e)

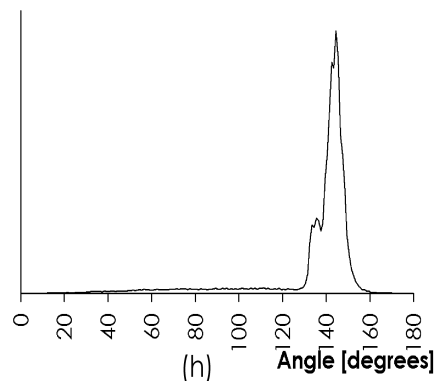

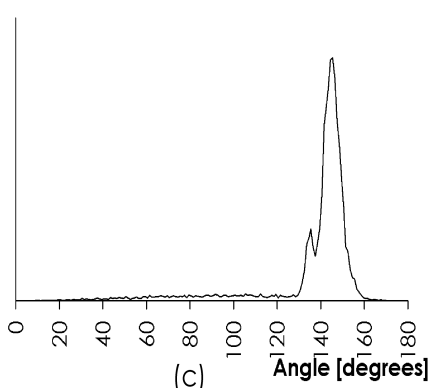
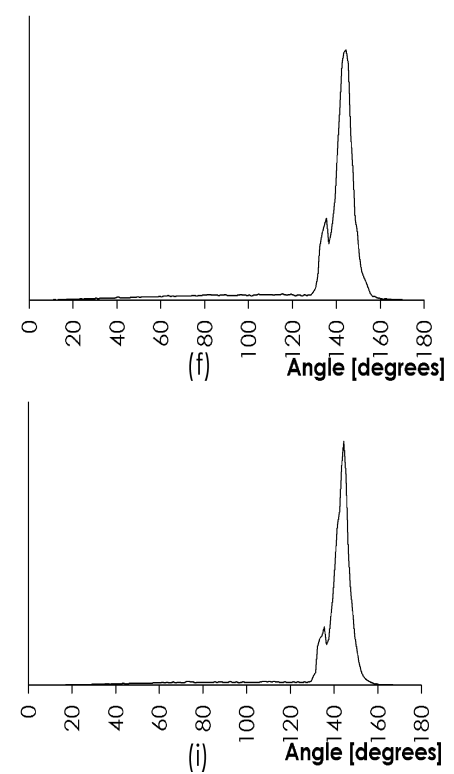

Figure 3.19: Si-O-Si angel distribution for samples generated for the study of the properties of silica aerogels and xerogels. (a) $\rho=2.2 \frac{\mathrm{g}}{\mathrm{cm}^{3}}$, (b) $\rho=1.49 \frac{\mathrm{g}}{\mathrm{cm}^{3}}$, (c) $\rho=1.05 \frac{\mathrm{g}}{\mathrm{cm}^{3}}$, (d) $\rho=0.77 \frac{\mathrm{g}}{\mathrm{cm}^{3}}$, (e) $\rho=0.58 \frac{\mathrm{g}}{\mathrm{cm}^{3}}$, (f) $\rho=0.45$ $\frac{\mathrm{g}}{\mathrm{cm}^{3}}$, (g) $\rho=0.35 \frac{\mathrm{g}}{\mathrm{cm}^{3}}$, (h) $\rho=0.28 \frac{\mathrm{g}}{\mathrm{cm}^{3}}$, (i) $\rho=0.23 \frac{\mathrm{g}}{\mathrm{cm}^{3}}$ 

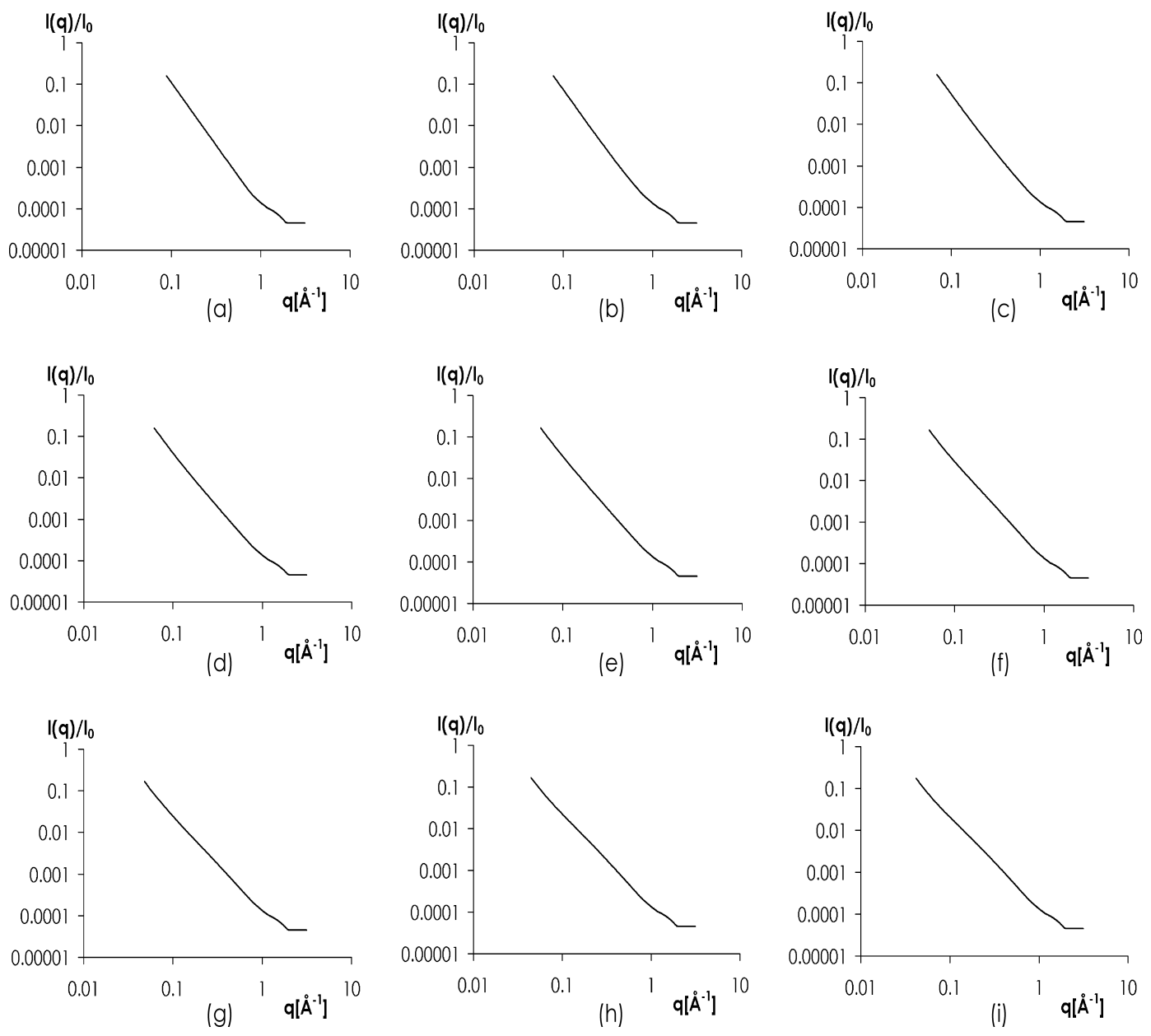

Figure 3.20: Scattering profiles of the samples generated for the study of the properties of silica aerogels and xerogels. (a) $\rho=2.2 \frac{\mathrm{g}}{\mathrm{cm}^{3}}$, (b) $\rho=1.49 \frac{\mathrm{g}}{\mathrm{cm}^{3}}$, (c) $\rho=1.05 \frac{\mathrm{g}}{\mathrm{cm}^{3}}$, (d) $\rho=0.77 \frac{\mathrm{g}}{\mathrm{cm}^{3}}$, (e) $\rho=0.58 \frac{\mathrm{g}}{\mathrm{cm}^{3}}$, (f) $\rho=0.45$ $\frac{\mathrm{g}}{\mathrm{cm}^{3}}$, (g) $\rho=0.35 \frac{\mathrm{g}}{\mathrm{cm}^{3}}$, (h) $\rho=0.28 \frac{\mathrm{g}}{\mathrm{cm}^{3}}$, (i) $\rho=0.23 \frac{\mathrm{g}}{\mathrm{cm}^{3}}$

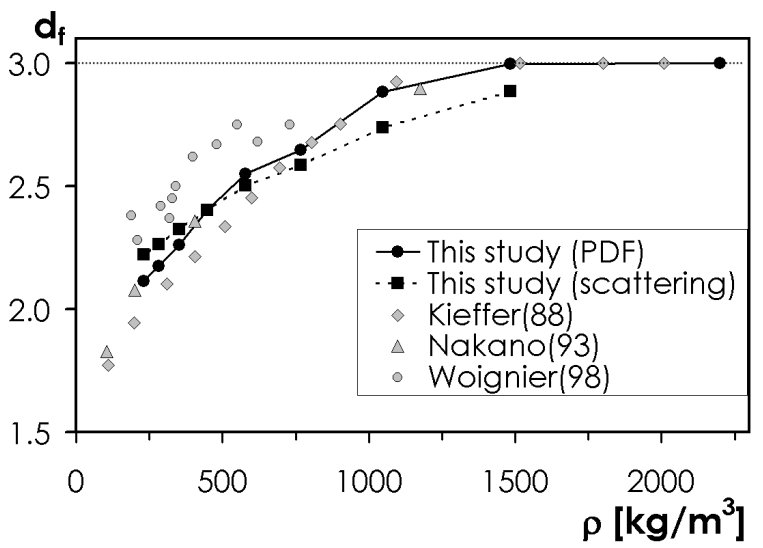

Figure 3.21: Fractal dimension of aerogel and xerogel samples used in this study 
small, between the particles, and a unique breaking point can not be identified.

For all the samples the strength was determined as the maximum stress recorded during the test. The elastic modulus and the Poisson ratio were determined by fitting the data corresponding to the initial strain (less than 1\%) to the Hooke's law as written in Eq. 3.13 and Eq. 3.14. The Poisson ratio for all the samples was around 0.2 which is comparable to the experimental value for dense silica glass, 0.17 [65]. The elastic modulus and the strength for the samples are summarized in the Table 3.3. This data is presented in the Fig. 3.24 along with experimental data taken from Refs. [8] and [10]. A power law can be used to describe the relationship between elastic modulus and density. The exponent characterizing that relation is $3.11 \pm 0.21$. Comparison of that value and previously published data is summarized in Table 3.4. Although the exponent determined here differs from the one determined by Campbell et al. [50, it is important to notice that the range of densities modeled here is broader and the exponent lies in the range of the values found experimentally, from 2.97 to 3.8. The range of densities modeled in this research covers almost the same range that is covered by Woignier et al. [8] for partially densified samples (last row in Table 3.4). In that range our exponent of $3.11 \pm 0.21$ compares well to the one determined experimentally of 3.2 .

The relation between strength and density is plotted in the Fig. 3.25. It can be seen the power law relationship between strength and density. The exponent for that relationship is $2.53 \pm 0.15$, a value that compares well with previously published values summarized in Table 3.5. There is a difference between the magnitude of the strength from experiments and simulations. The value of the strength for dense glass obtained in this study was $4.4 \mathrm{GPa}$ which is four times smaller than the experimental value of $18 \mathrm{GPa}$ reported in [55].

In conclusion, the direct expansion of crystalline samples of $\beta$-cristobalite to reach densities between $2.2 \frac{\mathrm{g}}{\mathrm{cm}^{3}}$ and $0.23 \frac{\mathrm{g}}{\mathrm{cm}^{3}}$, along with thermal processing, leads to fractal structures which allow to investigate the properties of silica aerogels and xerogels. The geometrical features of the modeled samples are characterized by the fractal dimension, determined from the PDF of the samples as well as from simulated scattering experiments. The results are found to be in good agreement with previous computational and experimental data. Furthermore, the mechanical properties, namely the elastic modulus and strength, of the porous samples are found to scale with density following a power law, which is expected for fractal structures. The exponent of those power law relations are $3.11 \pm 0.21$ and $2.53 \pm 0.15$ for elasticity and strength, respectively. Those values are in the range determined experimentally. The exponent for the elastic modulus vs. density relationship deviates only $3 \%$ from the experimental value determined by Woignier et al. [8] for partially densified aerogels in a similar range of densities as the samples modeled here. And the one for the strength vs. density relationship deviates $10 \%$ from the one determined by them. 


\begin{tabular}{ccc}
\hline Density $\left[\frac{\mathbf{g}}{\mathbf{c m}^{3}}\right]$ & $\mathbf{E}[\mathbf{G P a}]$ & $\sigma[\mathbf{M P a}]$ \\
\hline 2.20 & 63.0 & 4413 \\
\hline 1.49 & 24.3 & 2183 \\
\hline 1.05 & 8.5 & 843 \\
\hline 0.77 & 3.1 & 389 \\
\hline 0.58 & 1.1 & 157 \\
\hline 0.45 & 0.66 & 81 \\
\hline 0.35 & 0.26 & 52 \\
\hline 0.28 & 0.16 & 29 \\
\hline 0.23 & 0.05 & - \\
\hline
\end{tabular}

Table 3.3: Elastic moduli, E, and strength, $\sigma$, of silica aerogels and xerogels

\begin{tabular}{lll}
\hline Reference & Exponent & Density $\left[\frac{\mathrm{g}}{\mathbf{c m}^{3}}\right]$ \\
\hline This study & $3.11 \pm 0.21$ & $0.23-2.2$ \\
\hline Campbell et al. [50] & 3.45 & $1.67-2.2$ \\
\hline Groß et al. [12] & $3.49 \pm 0.07$ & $0.14-2.7$ \\
\hline Groß et al. [12] & $2.97 \pm 0.05$ & $0.08-1.2$ \\
\hline Woignier et al. [10] & 3.8 & $0.1-0.4$ (approx. values) \\
\hline Woignier et al. [8] & $3.7 \pm 0.2$ & $0.055-0.5$ (approx. values) \\
\hline Woignier et al. [8] & 3.2 & $0.42-2.2$ (approx. values) \\
\hline
\end{tabular}

Table 3.4: Exponent for the power law relationship between elastic modulus and density of porous silica

\begin{tabular}{lll}
\hline Reference & Exponent & Density $\left[\frac{\mathbf{g}}{\mathbf{c m}^{3}}\right]$ \\
\hline This study & $2.53 \pm 0.15$ & $0.23-2.2$ \\
\hline Woignier et al. $[8]$ & $2.6 \pm 0.2$ & $0.055-0.5$ (approx. values) \\
\hline Woignier et al. $[8]$ & 2.3 & $0.42-2.2$ (approx. values) \\
\hline
\end{tabular}

Table 3.5: Exponent for the power law relationship between strength and density of porous silica 


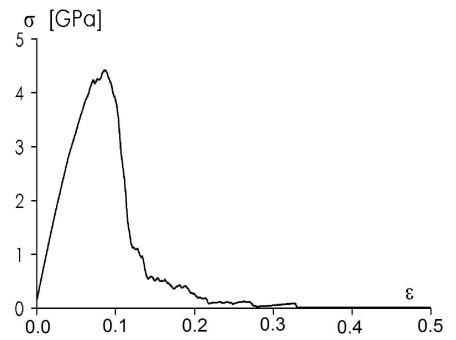

(a)

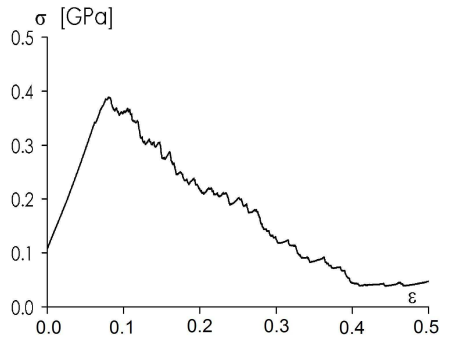

(d)

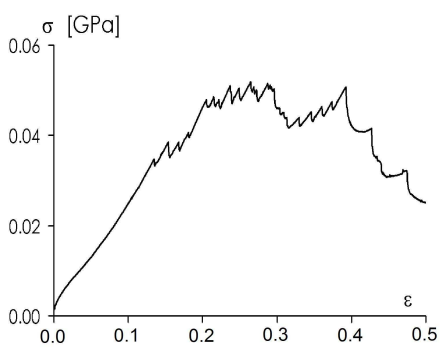

(g)

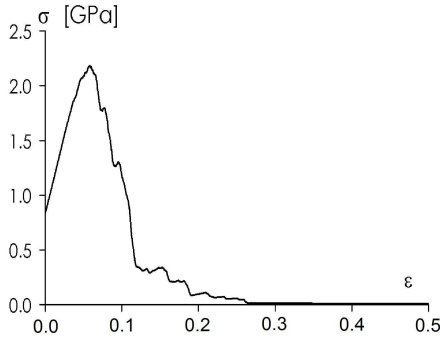

(b)

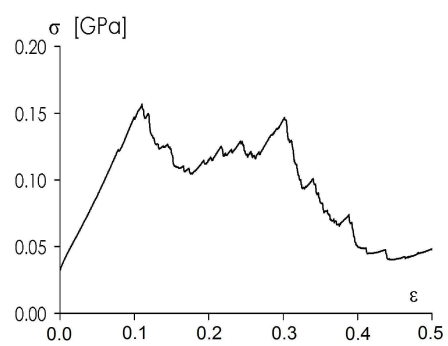

(e)

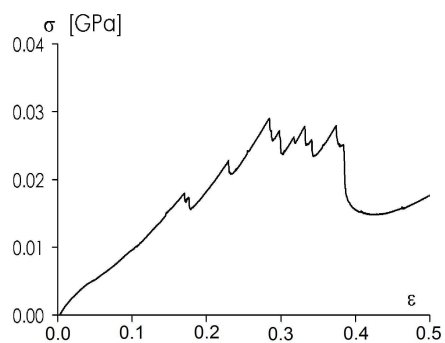

(h)

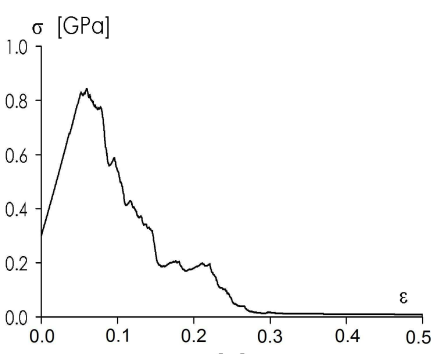

(c)

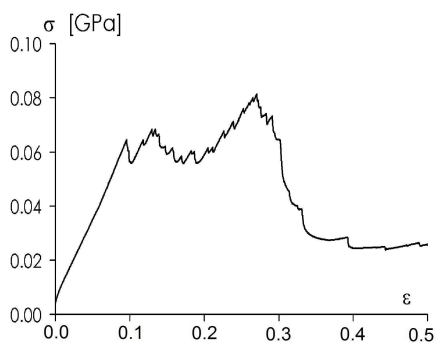

(f)

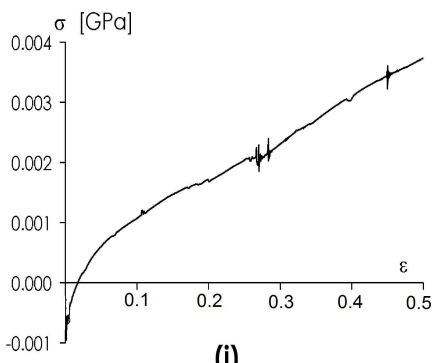

(i)

Figure 3.22: Stress vs. Strain plots of the samples generated for the study of the properties of silica aerogels and xerogels. (a) $\rho=2.2 \frac{\mathrm{g}}{\mathrm{cm}^{3}}$, (b) $\rho=1.49 \frac{\mathrm{g}}{\mathrm{cm}^{3}}$, (c) $\rho=1.05 \frac{\mathrm{g}}{\mathrm{cm}^{3}}$, (d) $\rho=0.77 \frac{\mathrm{g}}{\mathrm{cm}^{3}}$, (e) $\rho=0.58 \frac{\mathrm{g}}{\mathrm{cm}^{3}}$, (f) $\rho=0.45$ $\frac{\mathrm{g}}{\mathrm{cm}^{3}}$, (g) $\rho=0.35 \frac{\mathrm{g}}{\mathrm{cm}^{3}}$, (h) $\rho=0.28 \frac{\mathrm{g}}{\mathrm{cm}^{3}}$, (i) $\rho=0.23 \frac{\mathrm{g}}{\mathrm{cm}^{3}}$ 

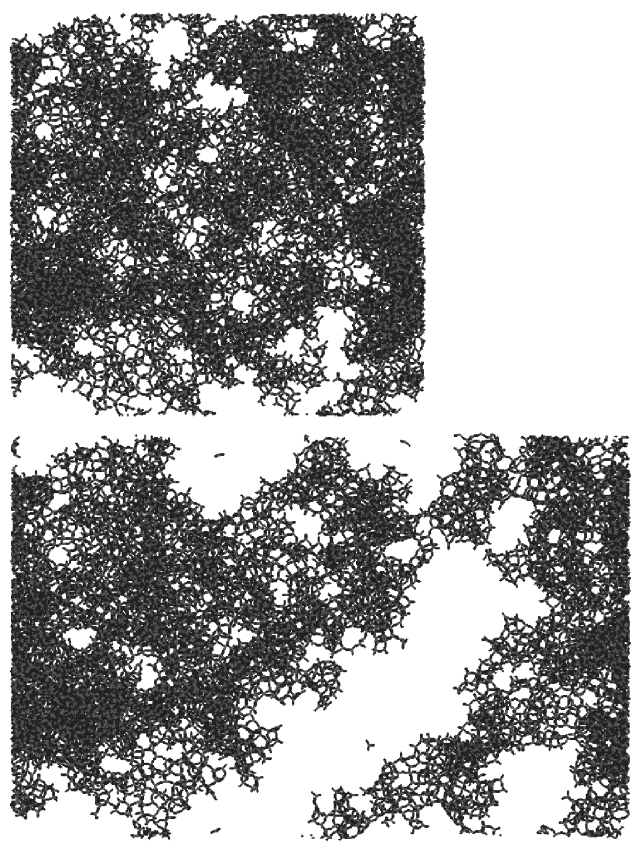

Figure 3.23: Sample of silica aerogel, $\rho=0.45 \frac{\mathrm{g}}{\mathrm{cm}^{3}}$, before (top) and after (bottom) a tension test 62

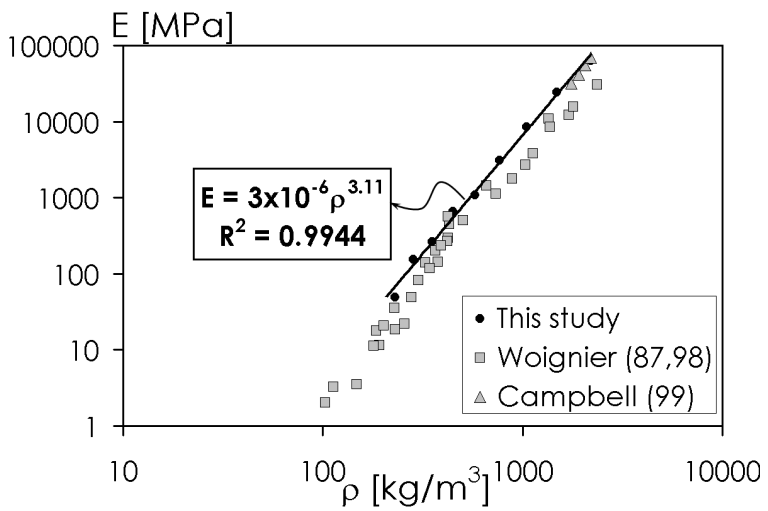

Figure 3.24: Elastic modulus vs. density for silica aerogels. Campbell $(99)[50]$, Woignier $(87,98)$ [8, 10].

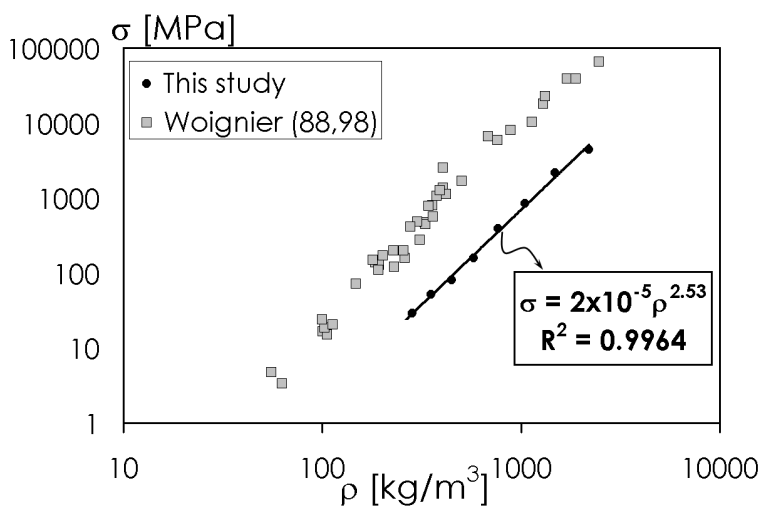

Figure 3.25: Strength vs. density for silica aerogels. Woignier $(88,98)[8,5]$. 


\section{Chapter 4}

\section{Molecular Dynamics Simulation Models for Hybrid Aerogels and Xerogels}

The objective of this chapter is to present the results of a model developed to study some of the structural and mechanical properties of hybrid aerogels and xerogels produced by adding methyl groups $\left(-\mathrm{CH}_{3}\right)$ to a silica structure. In the first section of the chapter the interatomic potentials used in the model are presented. Interatomic potentials are necessary to describe interactions between $S i, O, C$, and $H$ atoms. The interatomic potentials used were developed by Vashishta et al. [36, 46], Brenner et al. [66, 67], Boris et al. [68], and Dyson \&3 Smith [69]. Section [4.2 presents the procedure used to prepare the computational samples, including the implementation of Constraint Dynamics to model the presence of $\mathrm{CH}_{3}$ molecules in the silica structure. In section 4.3 the structural characteristics of hybrid aerogels and xerogels are compared to those of the silica samples having the same densities. In this section it is shown that the use of $\mathrm{CH}_{3}$-added silica to produce aerogels and xerogels leads to samples that do not display fractal features in the range of 9 to 30 A. Section 4.4 is dedicated to compare the mechanical properties of hybrid and silica aerogels and xerogels. It was found that by increasing the amount of $\mathrm{CH}_{3}$ in the samples their stiffness is reduced without affecting their strength, which leads to significant increases of the toughness of the material. It is concluded that $\mathrm{CH}_{3}$ molecules hinder the formation of $\mathrm{O}-\mathrm{Si}-\mathrm{O}$ bonds allowing the $\mathrm{Si}-\mathrm{O}$ bonds formed in the structure to stretch and rotate more easily. Modeling the $\mathrm{CH}_{3}$ molecules bonded to Si or $\mathrm{O}$ atoms yields identical structural or mechanical properties. 


\subsection{Interatomic Potentials for $\mathrm{CH}_{3}$-Added Systems}

Developing a Molecular Dynamics model for $\mathrm{CH}_{3}$-added silica samples requires the selection of interatomic potentials to cover all the possible interactions between the atoms in the system, namely $\mathrm{Si}, \mathrm{O}, \mathrm{C}$, and $\mathrm{H}$. For this research the interatomic potential developed by Vashishta et al. (See section 3.3) [36, 46] is used to describe the $\mathrm{Si}-\mathrm{O}, \mathrm{Si}-\mathrm{Si}$, and O-O interactions. The C-H, C-C, and $\mathrm{H}-\mathrm{H}$ interactions are modeled using the interatomic potential developed by Brenner et al. 66, 67. Interactions between $\mathrm{C}$ and $\mathrm{O}$ atoms, and between $\mathrm{H}$ and $\mathrm{O}$ atoms are described using the interatomic potential developed by Boris et al. [68]. And the interactions between $\mathrm{C}$ and $\mathrm{Si}$, and between $\mathrm{H}$ and $\mathrm{Si}$ atoms are modeled using the interatomic potential developed by Dyson \& Smith 69. In Table 4.1 the interatomic potentials used for each interaction are summarized. A detailed description of the potentials is presented below.

Brenner et al. [66, 67] potential is an empirical potential energy formulation developed to study solid carbon and hydrocarbon molecules. It has been successfully used in a multitude of MD applications involving polymers [70], carbon nanotubes [71], diamond structures [72, fracture dynamics [73], and nanoindentation processes [74]. The potential energy is given by a two-body function,

$$
V_{i j}=\sum_{i} \sum_{j}\left(V_{i j}^{(R)}-b_{i j} * V_{i j}^{(A)}\right)
$$

where $V_{i j}^{(R)}$ represents the repulsive energy, $V_{i j}^{(A)}$ represents the attractive energy, and $b_{i j}$ is the bond-order term for atoms $i$ and $j$.

The repulsive energy is given by

$$
V_{i j}^{(R)}=f^{c}\left(r_{i j}\right) *\left(1+\frac{Q_{i j}}{r_{i j}}\right) * A_{i j} * \exp \left(-\alpha * r_{i j}\right)
$$

where $r_{i j}$ is the distance between atoms $i$ and $j, Q_{i j}, A_{i j}$, and $\alpha_{i j}$ are empirical values obtained by fitting of structural data, and $f^{c}$ is a cutoff function defined as

$$
f^{c}\left(r_{i j}\right)= \begin{cases}1 & , r_{i j} \leq D_{i j}^{\min } \\ \frac{1}{2} *\left(1+\cos \left(\pi *\left(\frac{r_{i j}-D_{i j}^{\min }}{D_{i j}^{\max }-D_{i j}^{\min }}\right)\right)\right) & , D_{i j}^{\min }<r_{i j}<D_{i j}^{\max } \\ 0 & , r_{i j} \geq D_{i j}^{\max }\end{cases}
$$

where $D_{i j}^{\min }$ and $D_{i j}^{\max }$ are empirical parameters defining the extension of each type of interaction.

The attractive energy is given by

$$
V_{i j}^{(A)}=f^{c}\left(r_{i j}\right) * \sum_{n=1,3} B_{i j}^{(n)} * \exp \left(-\beta_{i j}^{(n)} * r_{i j}\right)
$$


where $B_{i j}^{(n)}$ and $\beta_{i j}^{(n)}$ are empirical parameters.

The bond-order function accounts for the coordination number and the bond angles of atoms $i$ and $j$, it is calculated using

$$
b_{i j}=\frac{1}{2}\left(p_{i j}^{\sigma \pi}+p_{j i}^{\sigma \pi}\right)+\pi_{i j}^{r c}+\pi_{i j}^{d h}
$$

where $p_{i j}^{\sigma \pi}$ is known as covalent bond interaction, and it is given by

$$
p_{i j}^{\sigma \pi}=\left(1+\sum_{k \neq i, j}\left(f^{c}\left(r_{i k}\right)+g_{i}\left(\cos \left(\theta_{j i k}\right)\right) * \exp \left(\lambda_{j i k}\right)+P_{i j}\right)\right)^{-\frac{1}{2}}
$$

$p_{i j}^{\sigma \pi}$ takes into account the atoms in the vicinity of the $i-j$ pair, and it was developed to represent the covalent interactions. $g_{i}$ is a function depending on the angle, $\theta_{j i k}$, formed between atoms $i, j$, and $k$, its value is estimated using splines interpolation from empirical data. $\lambda_{j i k}$ is an empirical negative screening parameter. $\pi_{i j}^{r c}$ and $\pi_{i j}^{d h}$ in Eq. 4.5 and $P_{i j}$ in Eq. 4.6 are empirical adjustment functions for the potential energy.

Ni et al. potential [68] for oxygen-hydrocarbons interactions was developed as an extension of Brenner et al. potential [66, 67]. It has the same form presented in Eq. 4.1, with the $V_{i j}^{(R)}$ and $V_{i j}^{(A)}$, and $b_{i j}$ given by Eq. 4.2, Eq. 4.4, and Eq. 4.5 respectively. The main difference is the form in which the function $g_{i}$ in Eq. 4.6] is obtained

$$
g_{i}\left(\theta_{j i k}\right)=a_{0}+a_{1} *\left(a_{2}-\cos \left(\theta_{j i k}\right)\right)
$$

where $a_{0}, a_{1}$, and $a_{2}$ are $(-0.014),(0.07)$, and $(-0.478)$ respectively, and $\theta_{j i k}$ is the angle formed between atoms $i, j$, and $k$.

Dyson et al. potential [69] for silicon-hydrocarbons interactions was developed as an extension of an earlier version of Brenner's potential for hydrocarbons 75 . The general form of the potential is presented in Eq. 4.1, but $V_{i j}^{(R)}, V_{i j}^{(A)}$, and $b_{i j}$ are given by

$$
\begin{gathered}
V_{i j}^{(R)}=f^{c}\left(r_{i j}\right) *\left(\frac{D_{i j}^{(e)}}{\left(S_{i j}-1\right)}\right) * \exp \left(-\sqrt{2 S_{i j}} * \beta_{i j}\left(r_{i j}-R_{i j}^{(e)}\right)\right) \\
V_{i j}^{(A)}=f^{c}\left(r_{i j}\right) *\left(\frac{D_{i j}^{(e)} S_{i j}}{\left(S_{i j}-1\right)}\right) * \exp \left(-\sqrt{\frac{2}{S_{i j}}} * \beta_{i j}\left(r_{i j}-R_{i j}^{(e)}\right)\right) \\
b_{i j}=\frac{1}{2}\left(p_{i j}^{-\delta_{i}}+p_{j i}^{-\delta_{j}}\right)
\end{gathered}
$$

where $D_{i j}^{(e)}, S_{i j}, \beta_{i j}, R_{i j}^{(e)}$, and $\delta_{i}$ are empirical parameters. $p_{i j}$ is calculated using 


\begin{tabular}{cc}
\hline Interaction & Source \\
\hline $\mathrm{Si}-\mathrm{Si}$ & Vashishta et al. [36, [46] \\
\hline $\mathrm{Si}-\mathrm{O}$ & Vashishta et al. [36,, 46$]$ \\
\hline $\mathrm{O}-\mathrm{O}$ & Vashishta et al. [36, [46] \\
\hline $\mathrm{C}-\mathrm{C}$ & Brenner et al. [66, 67] \\
\hline $\mathrm{C}-\mathrm{H}$ & Brenner et al. [66, 67] \\
\hline $\mathrm{H}-\mathrm{H}$ & Brenner et al. [66, [67] \\
\hline $\mathrm{O}-\mathrm{C}$ & Boris et al. [68] \\
\hline $\mathrm{O}-\mathrm{H}$ & Boris et al. [68] \\
\hline $\mathrm{Si}-\mathrm{C}$ & Dyson \& Smith [69] \\
\hline $\mathrm{Si}-\mathrm{H}$ & Dyson \& Smith [69] \\
\hline
\end{tabular}

Table 4.1: Interatomic potentials used for $\mathrm{CH}_{3}$-added systems

$$
p_{i j}=1+\sum_{k \neq i, j} f^{c}\left(r_{i k}\right) * g_{i}\left(\theta_{j i k}\right) * \exp \left(\alpha_{j i k}\left(\left(r_{i j}-R_{i j}^{(e)}\right)-\left(r_{i k}-R_{i k}^{(e)}\right)\right)\right)
$$

$\alpha_{j i k}$, and $R_{i j}^{(e)}$ are empirical parameters. The function $g_{i}\left(\theta_{j i k}\right)$ is given by

$$
g_{i}\left(\theta_{j i k}\right)=a_{0} *\left(1+\frac{c^{2}}{d^{2}}-\frac{c^{2}}{\left(d^{2}+\left(1+\cos \left(\theta_{j i k}\right)\right)^{2}\right)}\right)
$$

with $a_{0}, c$, and $d$ empirical parameters.

\subsection{Preparation of $\mathrm{CH}_{3}$-Added Systems}

The preparation of the computational samples of hybrid aerogels and xerogels started with the creation of dense silica glass samples following the procedure described in section 3.4. Those samples were equally expanded along the coordinate directions to match the desired density. The expansion was achieved multiplying the coordinates of the atoms by a factor given by

$$
\phi=\sqrt[3]{\frac{\rho_{0}}{\rho_{s}}}
$$

where $\rho_{0}$ and $\rho_{s}$ are the density of the dense glass $\left(2.2 \frac{\mathrm{g}}{\mathrm{cm}^{3}}\right)$ and the desired density of the porous sample, respectively.

In section 1.3.1 it was mentioned that $\mathrm{CH}_{3}$ molecules appear in the structure of the $\mathrm{CH}_{3}$-added silica mostly bonded to $\mathrm{Si}$ atoms, but they also can be bonded to $\mathrm{O}$ atoms. Groups of samples containing only $\mathrm{CH}_{3}$ bonded to Si atoms and only $\mathrm{CH}_{3}$ bonded to $\mathrm{O}$ atoms were created to study the effect of the presence of each type of molecule in the system. Molecules containing $\mathrm{CH}_{3}$ bonded to $\mathrm{Si}$ and bonded to $\mathrm{O}$ are shown in Fig. 4.1.

The expanded glass samples were used to produce five sets of samples containing $0 \mathrm{~mol} \% \mathrm{CH}_{3}$, 


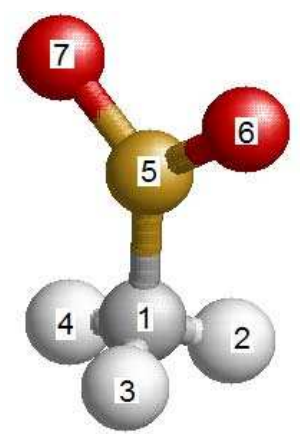

(a)

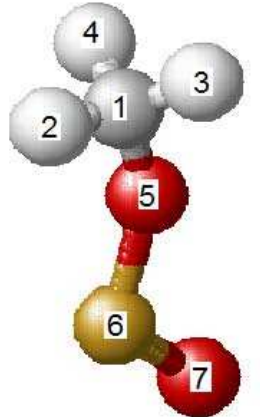

(b)

Figure 4.1: Type of $\mathrm{CH}_{3}-\mathrm{SiO}_{2}$ molecules 62. (a) $\mathrm{CH}_{3}$ molecule bonded to $\mathrm{Si}$, atom 1 is the $\mathrm{C}$ atom, atoms 2, 3, 4 are the $\mathrm{H}$ atoms, atom 5 is the $\mathrm{Si}$ atom, and atoms 6,7 are the $\mathrm{O}$ atoms. (b) $\mathrm{CH}_{3}$ molecule bonded to $\mathrm{O}$, atom 1 is the $\mathrm{C}$ atom, atoms 2, 3, 4 are the $\mathrm{H}$ atoms, atom 6 is the $\mathrm{Si}$ atom, and atoms 5,7 are the $\mathrm{O}$ atoms.

\begin{tabular}{lccc}
\hline & $\begin{array}{c}\mathbf{0} \mathbf{~ m o l \%} \\
\mathbf{C H}_{3}\end{array}$ & $\begin{array}{c}\mathbf{0 . 1} \mathbf{~ m o l \%} \\
\mathbf{C H}_{3}\end{array}$ & $\begin{array}{c}\mathbf{0 . 4} \mathbf{~ m o l \%} \\
\mathbf{C H}_{3}\end{array}$ \\
\hline Total number of atoms & 24000 & 24004 & 24012 \\
\hline Number of Si atoms & 8000 & 7060 & 5220 \\
\hline Number of O atoms & 16000 & 14120 & 10440 \\
\hline Number of C atoms & 0 & 706 & 2088 \\
\hline Number of H atoms & 0 & 2118 & 6264 \\
\hline
\end{tabular}

Table 4.2: Number of atoms used to create silica and hybrid samples

$0.1 \mathrm{~mol} \%$ and $0.4 \mathrm{~mol} \% \mathrm{CH}_{3}$ bonded to $\mathrm{Si}$ atoms, and $0.1 \mathrm{~mol} \%$ and $0.4 \mathrm{~mol} \% \mathrm{CH}_{3}$ bonded to $\mathrm{O}$ atoms. Samples containing $\mathrm{CH}_{3}$ were created randomly removing $\mathrm{SiO}_{2}$ molecules from the system, and randomly inserting $\mathrm{CH}_{3}-\mathrm{SiO}_{2}$ molecules. The amount of $\mathrm{SiO}_{2}$ removed and $\mathrm{CH}_{3}-\mathrm{SiO}_{2}$ added were chosen to keep the density of the original sample using a number of atoms close to 24000 , the same number of atoms used to create the silica aerogels and xerogels samples analyzed in [64 ( also see section 3.5). The number of atoms used for each group of samples is presented in Table 4.2. In Fig. 4.2 an example of expanded glass with $\mathrm{CH}_{3}-\mathrm{SiO}_{2}$ molecules added is presented.

Since $\mathrm{CH}_{3}-\mathrm{SiO}_{2}$ molecules are randomly inserted in the expanded glass, it is possible that some of the inserted particles are located too close to existing atoms in the system. If this happens the atoms will experience very large repulsive forces causing high accelerations and velocities. Adequately describing the trajectory of atoms moving with high velocity is difficult using explicit methods to solve the equations of motion (Eq. 3.15), e.g. Velocity-Verlet, and since the presence of atoms close to each other is only due to the procedure used to insert the $\mathrm{CH}_{3}-\mathrm{SiO}_{2}$ molecules, it is necessary to equilibrate the samples allowing the particles to gradually find equilibrium positions. The samples were thermalized for 10,000 time steps at $0 \mathrm{~K}$ in two stages defined as NVT ensembles, the time step for the simulations was $0.5 \mathrm{fs}$. The solution of the equations of motion was done using 


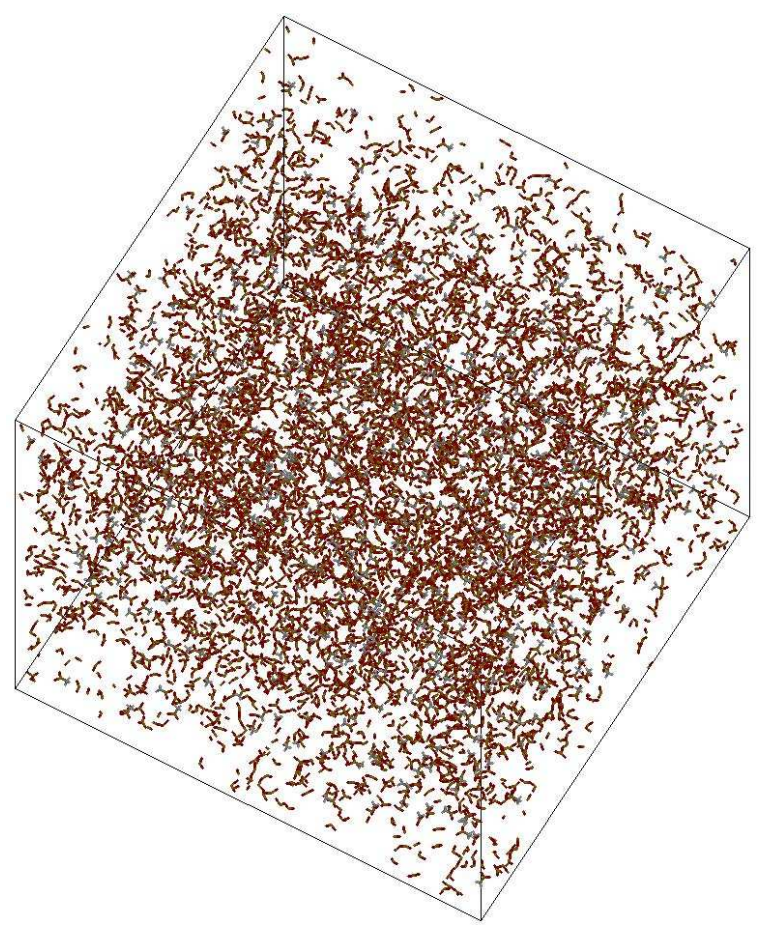

Figure 4.2: Expanded glass sample with $\mathrm{CH}_{3}-\mathrm{SiO}_{2}$ molecules added, density $0.28 \frac{g}{\mathrm{~cm}^{3}}[62$

high damping factors, $\gamma=100 \mathrm{ps}^{-1}$ during the first 5000 time steps, and $\gamma=10 \mathrm{ps}^{-1}$ the rest of the time.

After thermalizing the samples, they were processed to create the aerogel (or xerogel) structure. First, they were heated up to $1000 \mathrm{~K}$ using a heating rate of $20 \frac{\mathrm{K}}{\mathrm{ps}}$, and low damping factor $\gamma=$ $1 \mathrm{ps}^{-1}$, the same value used for the models of silica aerogels and xerogels described in chapter 3. Then, they were cooled down to $0 \mathrm{~K}$ using a cooling rate of $-20 \frac{K}{p s}$, and the same damping factor. The heating and cooling process were done in 120,000 time steps each, with $\delta t=0.5$ fs. The temperature profile used during the preparation of the samples is shown in Fig. 4.3 .

The procedure described above was used to create samples of densities $1.49,1.05,0.77,0.58$, $0.45,0.35$, and $0.28 \frac{\mathrm{g}}{\mathrm{cm}^{3}}$. For each density five different samples were created, namely $0 \mathrm{~mol} \% \mathrm{CH}_{3}$, $0.1 \mathrm{~mol} \%$ and $0.4 \mathrm{~mol} \% \mathrm{CH}_{3}$ bonded to Si atoms, and $0.1 \mathrm{~mol} \%$ and $0.4 \mathrm{~mol}_{\%} \mathrm{CH}_{3}$ bonded to $\mathrm{O}$ atoms. The samples have cubic shape and were modeled using periodic boundary conditions along all three Cartesian directions. The size of the samples is presented in Table 4.3.

The $\mathrm{CH}_{3}$ molecule and the atom to which it was bonded were modeled as a rigid molecule using Constraint Dynamics to guarantee that the $\mathrm{CH}_{3}$ molecule remains bonded to the desired atom through the simulation process. 


\begin{tabular}{|c|c|c|c|}
\hline $\begin{array}{c}\text { Density } \\
\left(\frac{g}{c m^{3}}\right)\end{array}$ & $\begin{array}{c}\mathrm{CH}_{3} \text { content } \\
\mathrm{mol} \%\end{array}$ & $\begin{array}{c}\mathrm{CH}_{3} \\
\text { bonded to }\end{array}$ & $\begin{array}{c}\text { Volume } \\
\AA^{3}\end{array}$ \\
\hline \multirow[t]{5}{*}{1.49} & 0.0 & - & $(81.3)^{3}$ \\
\hline & 0.1 & $\mathrm{Si}$ & $(78.6)^{3}$ \\
\hline & 0.1 & $\mathrm{O}$ & $(78.6)^{3}$ \\
\hline & 0.4 & $\mathrm{Si}$ & $(72.8)^{3}$ \\
\hline & 0.4 & $\mathrm{O}$ & $(72.8)^{3}$ \\
\hline \multirow[t]{5}{*}{1.05} & 0.0 & - & $(91.3)^{3}$ \\
\hline & 0.1 & $\mathrm{Si}$ & $(88.3)^{3}$ \\
\hline & 0.1 & $\mathrm{O}$ & $(88.3)^{3}$ \\
\hline & 0.4 & $\mathrm{Si}$ & $(81.8)^{3}$ \\
\hline & 0.4 & $\mathrm{O}$ & $(81.8)^{3}$ \\
\hline \multirow{5}{*}{0.77} & 0.0 & - & $(101.3)^{3}$ \\
\hline & 0.1 & $\mathrm{Si}$ & $(98.0)^{3}$ \\
\hline & 0.1 & $\mathrm{O}$ & $(98.0)^{3}$ \\
\hline & 0.4 & $\mathrm{Si}$ & $(90.7)^{3}$ \\
\hline & 0.4 & $\mathrm{O}$ & $(90.7)^{3}$ \\
\hline \multirow[t]{5}{*}{0.58} & 0.0 & - & $(111.3)^{3}$ \\
\hline & 0.1 & $\mathrm{Si}$ & $(107.6)^{3}$ \\
\hline & 0.1 & $\mathrm{O}$ & $(107.6)^{3}$ \\
\hline & 0.4 & $\mathrm{Si}$ & $(99.7)^{3}$ \\
\hline & 0.4 & $\mathrm{O}$ & $(99.7)^{3}$ \\
\hline \multirow[t]{5}{*}{0.45} & 0.0 & - & $(121.3)^{3}$ \\
\hline & 0.1 & $\mathrm{Si}$ & $(117.3)^{3}$ \\
\hline & 0.1 & $\mathrm{O}$ & $(117.3)^{3}$ \\
\hline & 0.4 & $\mathrm{Si}$ & $(108.6)^{3}$ \\
\hline & 0.4 & $\mathrm{O}$ & $(108.6)^{3}$ \\
\hline \multirow[t]{5}{*}{0.35} & 0.0 & - & $(131.3)^{3}$ \\
\hline & 0.1 & $\mathrm{Si}$ & $(127.0)^{3}$ \\
\hline & 0.1 & $\mathrm{O}$ & $(127.0)^{3}$ \\
\hline & 0.4 & $\mathrm{Si}$ & $(117.6)^{3}$ \\
\hline & 0.4 & $\mathrm{O}$ & $(117.6)^{3}$ \\
\hline \multirow[t]{5}{*}{0.28} & 0.0 & - & $(141.3)^{3}$ \\
\hline & 0.1 & $\mathrm{Si}$ & $(135.5)^{3}$ \\
\hline & 0.1 & $\mathrm{O}$ & $(135.5)^{3}$ \\
\hline & 0.4 & $\mathrm{Si}$ & $(126.5)^{3}$ \\
\hline & 0.4 & $\mathrm{O}$ & $(126.5)^{3}$ \\
\hline
\end{tabular}

Table 4.3: Density and volume of silica and hybrid aerogel and xerogel samples 


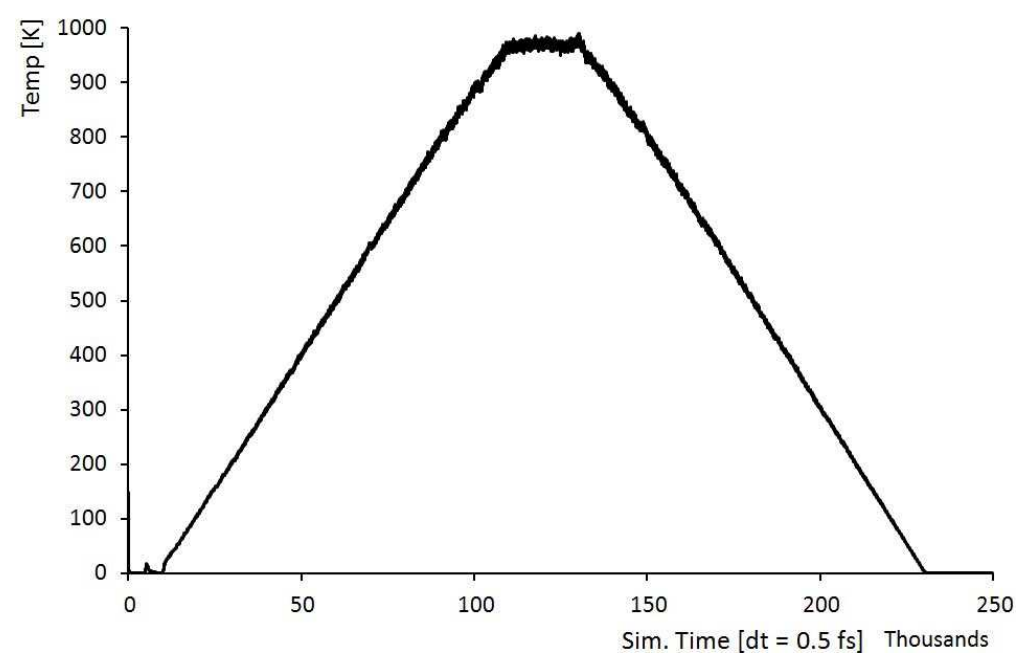

Figure 4.3: Temperature profile used to create silica and hybrid aerogel and xerogel samples

\subsubsection{Constraint Dynamics applied to $\mathrm{CH}_{3}-\mathrm{SiO}_{2}$ molecules}

The fundamental concepts of Constraint Dynamics were presented in section 2.1.5. They are applied in this research to model a rigid molecule formed by a $\mathrm{CH}_{3}$ molecule bonded to either a $\mathrm{Si}$ or $\mathrm{O}$ atom. The atoms forming the rigid molecule are identified as atoms 1, 2, 3, 4, and 5 in Fig. 4.1. where atom 1 is the $\mathrm{C}$ atom, atoms 2,3 , and 4 are the $\mathrm{H}$ atoms, and atom 5 is either the $\mathrm{Si}$ or the $\mathrm{O}$ atom. The constraints applied to the system are the distances between the $\mathrm{C}$ atom and the $\mathrm{H}$ atoms, i.e. $d_{12}, d_{13}$, and $d_{14}$, the distance between the $\mathrm{C}$ atom and the $\mathrm{Si}$ or $\mathrm{O}$ atom, i.e. $d_{15}$, and the distances between the $\mathrm{Si}$ or $\mathrm{O}$ atom and the $\mathrm{H}$ atoms, i.e. $d_{52}, d_{53}$, and $d_{54}$. The set of constraints allows the molecule to rotate about the $\mathrm{C}-\mathrm{Si}$, or $\mathrm{C}-\mathrm{O}$, bond while keeping the bonding angles constant, i.e. $\widehat{\mathrm{H}-\mathrm{C}-\mathrm{H}}, \widehat{\mathrm{H}-\mathrm{C}-\mathrm{Si}}$, and $\widehat{\mathrm{H}-\mathrm{C}-\mathrm{O}}$.

The geometrical characteristics of the $\mathrm{CH}_{3}$ bonded to $\mathrm{Si}$ and the $\mathrm{CH}_{3}$ bonded to $\mathrm{O}$ molecules were defined using the methane molecule, $\mathrm{CH}_{4}$, as starting point. The atomic structure of $\mathrm{CH}_{4}$ is tetrahedral, with the $\mathrm{C}$ atom in the centroid of the tetrahedron and the four $\mathrm{H}$ atoms in the corners. The bonding distance between $\mathrm{C}$ and $\mathrm{H}$ is about $1.1 \AA$ and the bonding angle $\widehat{\mathrm{H}-\mathrm{C}-\mathrm{H}}$ is 109.5 $5^{\circ}$. The $\mathrm{CH}_{3}$ - bonded to $\mathrm{Si}$ and $\mathrm{CH}_{3}$ bonded to $\mathrm{O}$ molecules were formed by replacing one of the $\mathrm{H}$ atoms of the $\mathrm{CH}_{4}$ molecule, and using bonding distances $\mathrm{Si}-\mathrm{C}$ and $\mathrm{O}-\mathrm{C}$ as $1.9 \AA$ and $1.3 \AA$ respectively [68, 76, 77].

The seven constraint equations necessary to model $\mathrm{CH}_{3}$ bonded to $\mathrm{Si}$ and $\mathrm{CH}_{3}$ bonded to $\mathrm{O}$ molecules are defined applying Eq. 2.13

$$
\begin{aligned}
& \psi_{12}(t)=\left(r_{12}(t)\right)^{2}-\left(d_{C H}\right)^{2}=0 \\
& \psi_{13}(t)=\left(r_{13}(t)\right)^{2}-\left(d_{C H}\right)^{2}=0
\end{aligned}
$$




$$
\begin{aligned}
& \psi_{14}(t)=\left(r_{14}(t)\right)^{2}-\left(d_{C H}\right)^{2}=0 \\
& \psi_{15}(t)=\left(r_{15}(t)\right)^{2}-\left(d_{C X}\right)^{2}=0 \\
& \psi_{52}(t)=\left(r_{52}(t)\right)^{2}-\left(d_{X H}\right)^{2}=0 \\
& \psi_{53}(t)=\left(r_{53}(t)\right)^{2}-\left(d_{X H}\right)^{2}=0 \\
& \psi_{54}(t)=\left(r_{54}(t)\right)^{2}-\left(d_{X H}\right)^{2}=0
\end{aligned}
$$

where $\psi_{i j}(t)$ is the constraint function, $r_{i j}(t)$ is the distance between atoms $i$ and $j$ at the time step $(t), d_{i j}$ is the desired distance between atoms $i$ and $j$, and the subindex $X$ applies for the type of atom corresponding to atom 5, i.e. Si or O.

The constraint forces are obtained using Eq. 2.14

$$
\begin{gathered}
\vec{g}_{1}(t)=\lambda_{12} * \vec{r}_{12}(t)+\lambda_{13} * \vec{r}_{13}(t)+\lambda_{14} * \vec{r}_{14}(t)+\lambda_{15} * \vec{r}_{15}(t) \\
\vec{g}_{2}(t)=-\lambda_{12} * \vec{r}_{12}(t)-\lambda_{52} * \vec{r}_{52}(t) \\
\vec{g}_{3}(t)=-\lambda_{13} * \vec{r}_{13}(t)-\lambda_{53} * \vec{r}_{53}(t) \\
\vec{g}_{4}(t)=-\lambda_{14} * \vec{r}_{14}(t)-\lambda_{54} * \vec{r}_{54}(t) \\
\vec{g}_{5}(t)=\lambda_{52} * \vec{r}_{52}(t)+\lambda_{53} * \vec{r}_{53}(t)+\lambda_{54} * \vec{r}_{54}(t)-\lambda_{15} * \vec{r}_{15}(t)
\end{gathered}
$$

where $\vec{g}_{i}(t)$ is the constraint force on atom $i$ at the time step $(\mathrm{t}), \lambda_{i j}$ is the Lagrangian multiplier corresponding to the constraint force between atoms $i$ and $j$, and $\vec{r}_{i j}(t)$ is the vector position from atom $i$ to atom $j$ at the time step $(t)$.

The positions at time step $(t+\delta t)$ are calculated using Eq. 2.15

$$
\begin{gathered}
\vec{r}_{1}(t+\delta t)=\vec{r}_{1 \mathrm{uc}}(t+\delta t)+C_{1} * \lambda_{12} * \vec{r}_{12}(t)+C_{1} * \lambda_{13} * \vec{r}_{13}(t) \ldots \\
\cdots+C_{1} * \lambda_{14} * \vec{r}_{14}(t)+C_{1} * \lambda_{15} * \vec{r}_{15}(t) \\
\vec{r}_{2}(t+\delta t)=\vec{r}_{2 \mathrm{uc}}(t+\delta t)-C_{2} * \lambda_{12} * \vec{r}_{12}(t)-C_{2} * \lambda_{52} * \vec{r}_{52}(t) \\
\vec{r}_{3}(t+\delta t)=\vec{r}_{3 \mathrm{uc}}(t+\delta t)-C_{3} * \lambda_{13} * \vec{r}_{13}(t)-C_{3} * \lambda_{53} * \vec{r}_{53}(t) \\
\vec{r}_{4}(t+\delta t)=\vec{r}_{4 \mathrm{uc}}(t+\delta t)-C_{4} * \lambda_{14} * \vec{r}_{14}(t)-C_{4} * \lambda_{54} * \vec{r}_{54}(t) \\
\vec{r}_{5}(t+\delta t)=\vec{r}_{5 \mathrm{uc}}(t+\delta t)+C_{5} * \lambda_{52} * \vec{r}_{52}(t)+C_{5} * \lambda_{53} * \vec{r}_{53}(t) \ldots \\
\ldots+C_{5} * \lambda_{54} * \vec{r}_{54}(t)-C_{5} * \lambda_{15} * \vec{r}_{15}(t)
\end{gathered}
$$

where $\vec{r}_{i \mathrm{uc}}(t+\delta t)$ is the vector position of atom $i$ calculated using only the forces due to the interatomic potential, i.e. without the effect of the constraint forces, $C_{i}$ is a constant defined as 


$$
C_{i}=\left(\frac{(\delta t)^{2}}{m_{i}}\right)
$$

where $m_{i}$ is the mass of atom $i$.

To solve Eq. 4.16 is necessary to find the seven Lagrangian multipliers, i.e. $\lambda_{12}, \lambda_{13}, \lambda_{14}, \lambda_{15}$, $\lambda_{52}, \lambda_{53}, \lambda_{54}$. The relative position vector between atoms $i$ and $j$ at any time during the simulation is given by

$$
\vec{r}_{i j}(t)=\vec{r}_{i}(t)-\vec{r}_{j}(t)
$$

Using Eq. 4.16 and Eq. 4.18 seven equations involving relative position vector between the atoms in the molecule can be written

$$
\begin{gathered}
\vec{r}_{12}(t+\delta t)=\vec{r}_{12 \mathrm{uc}}(t+\delta t)+C_{12} * \lambda_{12} * \vec{r}_{12}(t)+C_{1} * \lambda_{13} * \vec{r}_{13}(t)+\ldots \\
\ldots C_{1} * \lambda_{14} * \vec{r}_{14}(t)+C_{1} * \lambda_{15} * \vec{r}_{15}(t)+C_{2} * \lambda_{52} * \vec{r}_{52}(t) \\
\vec{r}_{13}(t+\delta t)=\vec{r}_{13 \mathrm{uc}}(t+\delta t)+C_{1} * \lambda_{12} * \vec{r}_{12}(t)+C_{13} * \lambda_{13} * \vec{r}_{13}(t)+\ldots \\
\ldots C_{1} * \lambda_{14} * \vec{r}_{14}(t)+C_{1} * \lambda_{15} * \vec{r}_{15}(t)+C_{3} * \lambda_{53} * \vec{r}_{53}(t) \\
\vec{r}_{14}(t+\delta t)=\vec{r}_{14 \mathrm{uc}}(t+\delta t)+C_{1} * \lambda_{12} * \vec{r}_{12}(t)+C_{1} * \lambda_{13} * \vec{r}_{13}(t)+\ldots \\
\ldots C_{14} * \lambda_{14} * \vec{r}_{14}(t)+C_{1} * \lambda_{15} * \vec{r}_{15}(t)+C_{4} * \lambda_{54} * \vec{r}_{54}(t) \\
\vec{r}_{15}(t+\delta t)=\vec{r}_{15 \mathrm{uc}}(t+\delta t)+C_{1} * \lambda_{12} * \vec{r}_{12}(t)+C_{1} * \lambda_{13} * \vec{r}_{13}(t)+\ldots \\
\ldots C_{1} * \lambda_{14} * \vec{r}_{14}(t)+C_{15} * \lambda_{15} * \vec{r}_{15}(t)-C_{5} * \lambda_{52} * \vec{r}_{52}(t) \ldots \\
\ldots-C_{5} * \lambda_{53} * \vec{r}_{53}(t)-C_{5} * \lambda_{54} * \vec{r}_{54}(t) \ldots \\
\vec{r}_{54}(t+\delta t)=\vec{r}_{54 \mathrm{uc}}(t+\delta t)-C_{5} * \lambda_{15} * \vec{r}_{15}(t)+C_{5} * \lambda_{52} * \vec{r}_{52}(t)+\ldots \\
\vec{r}_{53}(t+\delta t)=\vec{r}_{53 \mathrm{uc}}(t+\delta t)-C_{5} * \lambda_{15} * \vec{r}_{15}(t)+C_{5} * \lambda_{52} * \vec{r}_{52}(t)+\ldots \\
\vec{r}_{52}(t+\delta t)=\vec{r}_{52 \mathrm{uc}}(t+\delta t)-C_{5} * \lambda_{15} * \vec{r}_{15}(t)+C_{52} * \lambda_{52} * \vec{r}_{52}(t)+\ldots \\
\ldots C_{53} * \lambda_{53} * \vec{r}_{53}(t)+C_{53} * \lambda_{54} * \vec{r}_{54}(t)+C_{2} * \lambda_{12} * \vec{r}_{12}(t)+C_{5} * \lambda_{54} * \vec{r}_{54}(t)+C_{3} * \lambda_{13} * \vec{r}_{13}(t) \\
\ldots
\end{gathered}
$$




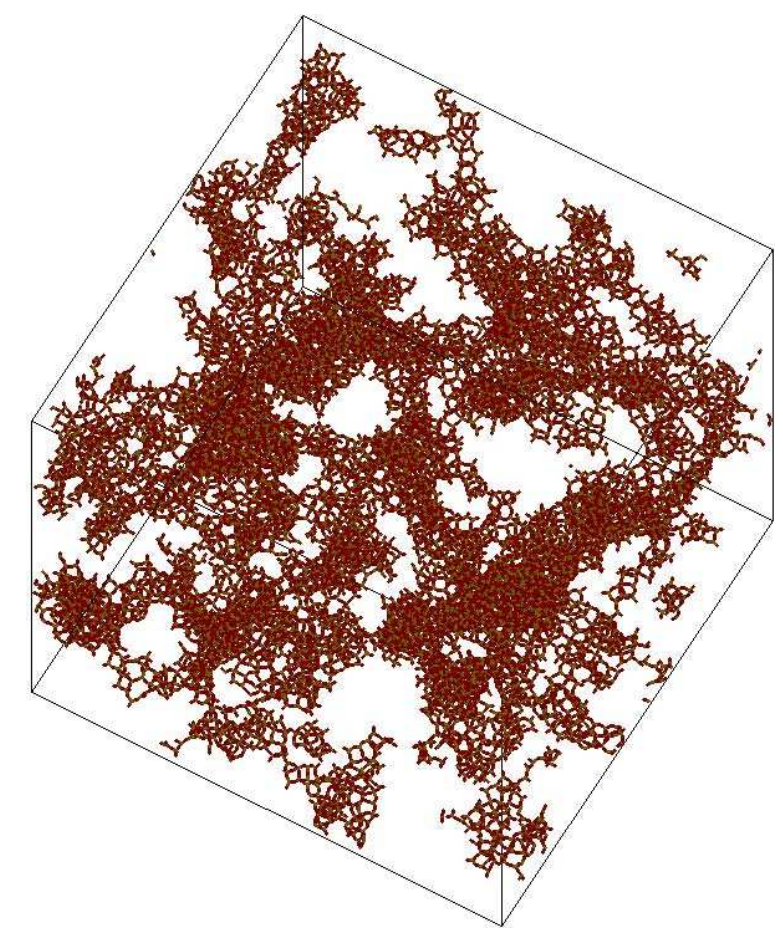

Figure 4.4: Silica aerogel sample, density $0.28 \frac{\mathrm{g}}{\mathrm{cm}^{3}}$, before tension test 62

$$
\ldots C_{5} * \lambda_{53} * \vec{r}_{53}(t)+C_{54} * \lambda_{54} * \vec{r}_{54}(t)+C_{4} * \lambda_{14} * \vec{r}_{14}(t)
$$

with $C_{i j}=C_{i}+C_{j}$

Since the magnitude of the seven vectors in Eq. 4.19 must satisfy the constraints defined in Eq. 4.14 there is a $7 \times 7$ system of quadratic equations to calculate the seven Lagrangian multipliers. A new set of Lagrangian multipliers must be found each time the positions of the atoms in the system are evolved.

\subsection{Structural Characterization of $\mathrm{CH}_{3}$-Added Systems}

Samples of silica and hybrid aerogels of density $0.28 \frac{\mathrm{g}}{\mathrm{cm}^{3}}$, with $0 \mathrm{~mol} \%, 0.1 \mathrm{~mol} \%$ and $0.4 \mathrm{~mol} \%$ $\mathrm{CH}_{3}$ content, are presented in Figs. 4.4- 4.6, It is noticeable that the presence of $\mathrm{CH}_{3}$ produced changes in the structure of the aerogel sample. The samples containing $\mathrm{CH}_{3}$ are characterized by the presence of smaller clusters and smaller pores than those of pure silica. This observation is in agreement with experimental findings indicating that hybrid aerogels prepared using mixtures of tetramethoxysilane (TMOS) and trimethoxymethylsilane (TRIMOS) have larger surface area, and smaller average pore size than those prepare with TMOS as only precursor. The presence of $\mathrm{CH}_{3}$ leads to the formation of more branched and open structures [18]. Figures corresponding to the additional samples are presented in appendix $\mathrm{A}$. 

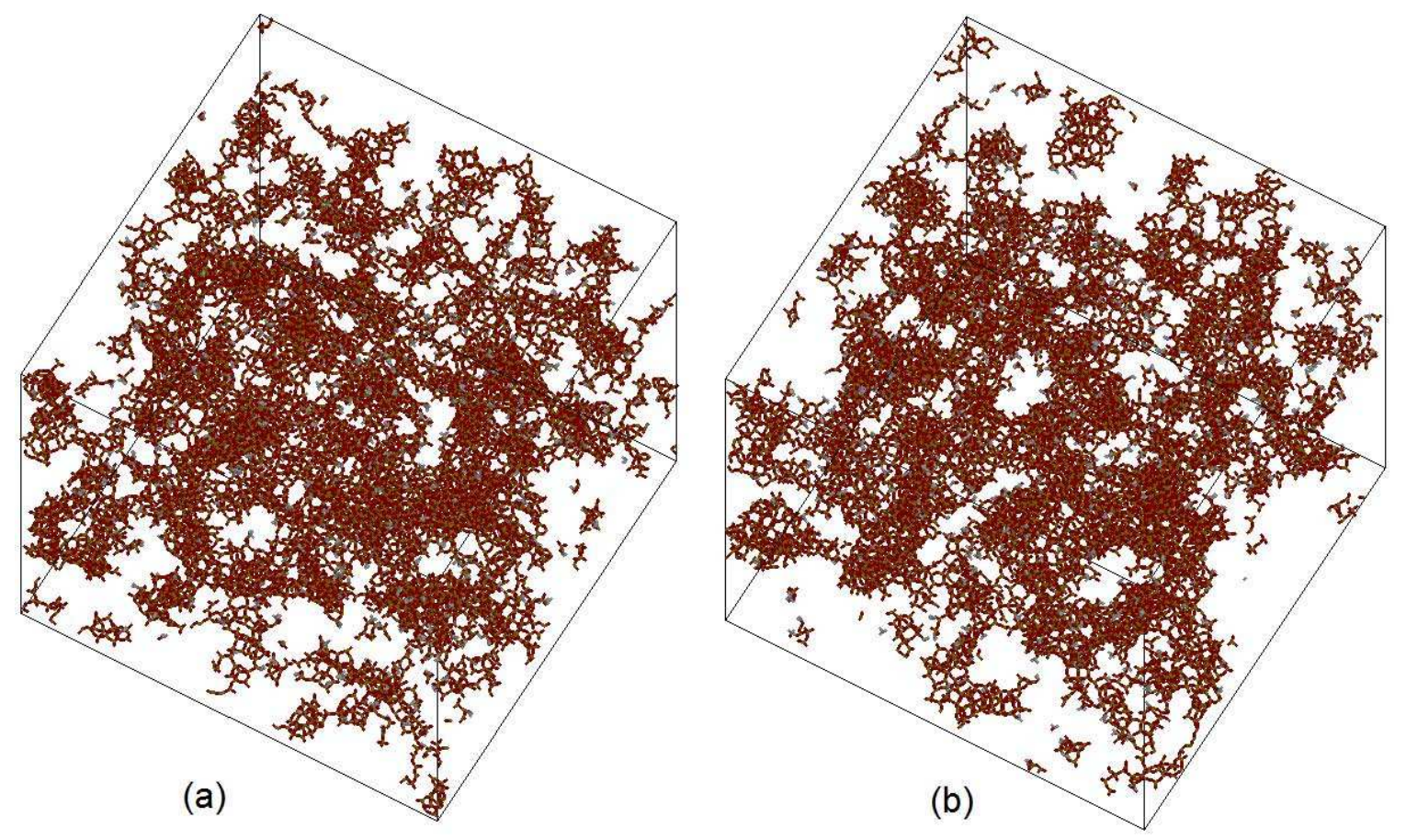

Figure 4.5: Aerogel sample with $0.1 \mathrm{~mol} \% \mathrm{CH}_{3}$ content, density $0.28 \frac{\mathrm{g}}{\mathrm{cm}^{3}}$, before tension test [62]. (a) $\mathrm{CH}_{3}$ bonded to $\mathrm{Si}$, (b) $\mathrm{CH}_{3}$ bonded to $\mathrm{O}$.
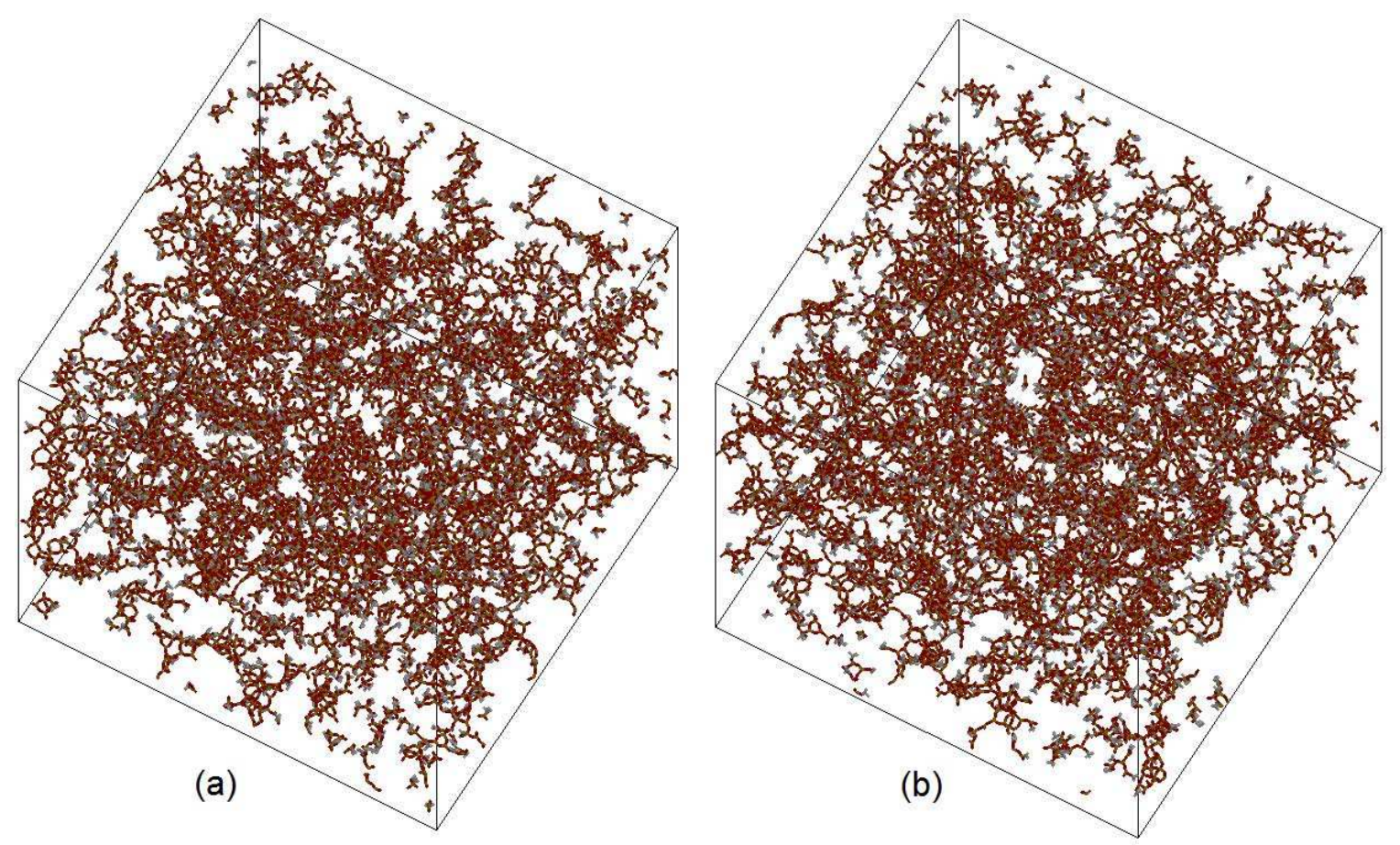

Figure 4.6: Aerogel sample with $0.4 \mathrm{~mol} \% \mathrm{CH}_{3}$ content, density $0.28 \frac{\mathrm{g}}{\mathrm{cm}^{3}}$, before tension test 62]. (a) $\mathrm{CH}_{3}$ bonded to $\mathrm{Si}$, (b) $\mathrm{CH}_{3}$ bonded to $\mathrm{O}$. 


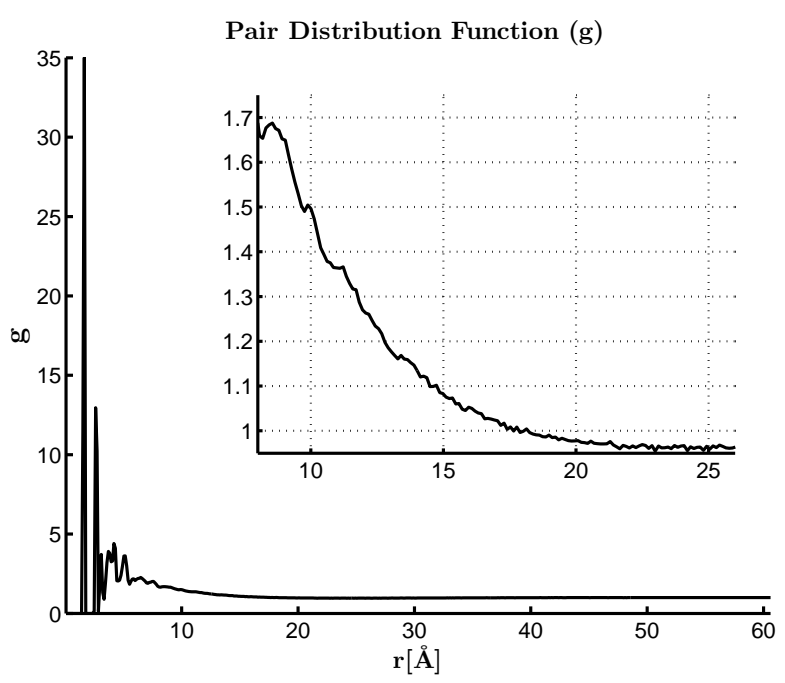

(a)

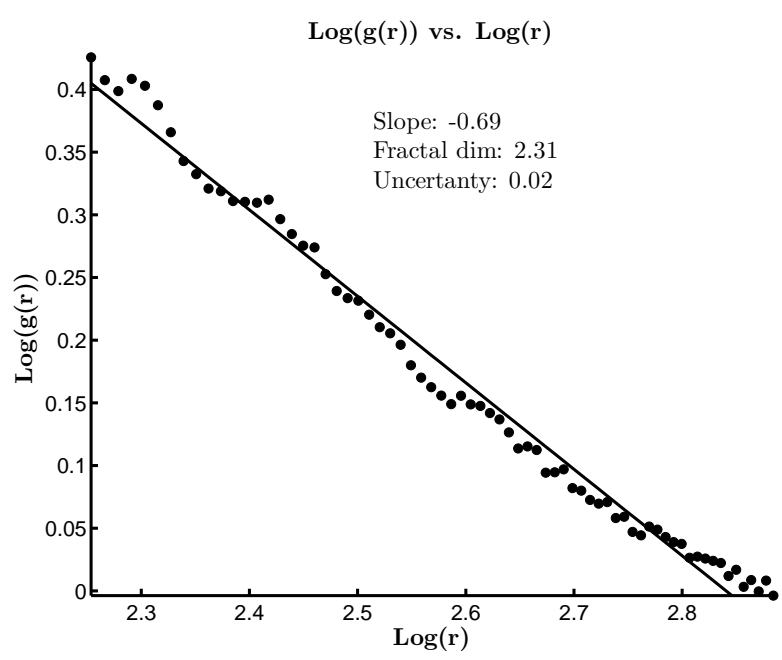

(b)

Figure 4.7: (a) Pair Distribution Function, (b) fractal dimension for silica aerogel, $\rho=0.45 \frac{\mathrm{g}}{\mathrm{cm}^{3}}$.

In Figs. 4.7 - 4.10 the Pair Distribution Functions (PDF) for samples of silica and hybrid aerogels of density $0.45 \frac{\mathrm{g}}{\mathrm{cm}^{3}}$ are illustrated. The inset in those figures displays the intermediate distance range, where the fractal character of the structure is manifested [41].

The addition of $\mathrm{CH}_{3}$ causes a reduction, and eventual elimination, of the fractal features in the structure of the aerogel. For a sample of pure silica (Fig. 4.7) the fractal features are observed in a region between 9.5 and $18 \AA$ approximately, while for samples containing $0.1 \mathrm{~mol} \% \mathrm{CH}_{3}$ (Figs. 4.8- 4.9) that region extends only between 9 and $12 \AA$ approximately, and for samples containing $0.4 \mathrm{~mol}_{0} \mathrm{CH}_{3}$ (Fig. 4.10) the fractal region has disappeared all together.

The (b) parts in Figs. 4.7-4.9 illustrate how the fractal dimension of the samples is estimated by representing the PDF in a $\log -\log$ plot, and applying Eq. 2.37. The fractal dimension for the pure silica sample is $2.31 \pm 0.02$, and for hybrid samples containing $0.1 \mathrm{~mol}^{\circ} \mathrm{CH}_{3}$ it is virtually the same, $2.33 \pm 0.08$ and $2.25 \pm 0.09$, indicating that the addition of $\mathrm{CH}_{3}$ only alters the extension of the fractal region, and not the value of the fractal dimension. Plots illustrating the PDF and the fractal dimension for all the additional samples are included in the appendix B,

The fractal dimension of the silica samples produced for this study is plotted in Fig. 4.11, along with data reported in [64]. The samples produced for this study have similar fractal dimensions and fractal dimension vs. density relationship than those reported in [64]. The procedures used in both cases are different, in [64 the samples were started expanding the atomic positions of a crystalline phase, then they were thermally treated to generate the fractal structures (also see section 3.5). While for this study the atomic positions of glass samples were expanded to the desired density, then thermally treated. The thermal treatments used to prepare the samples for each study were 


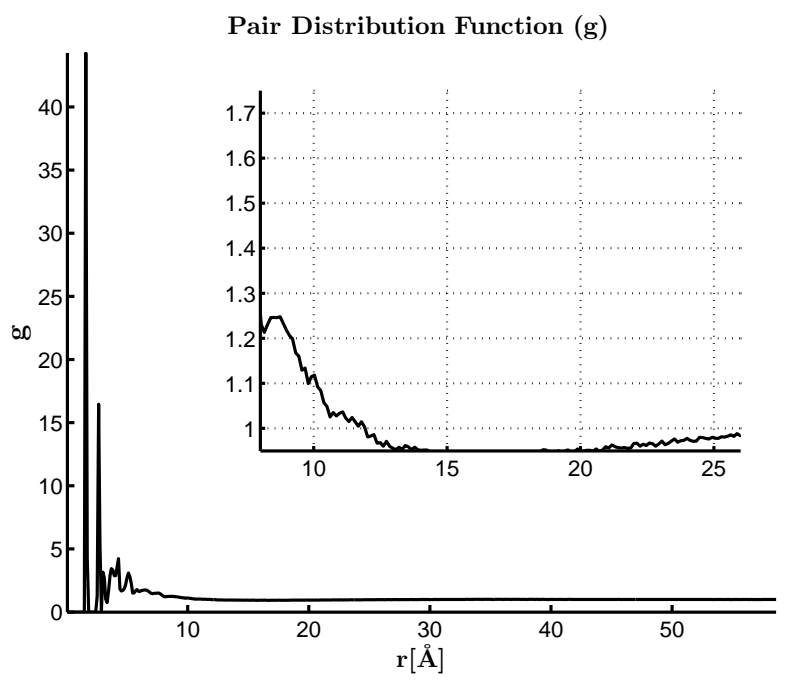

(a)

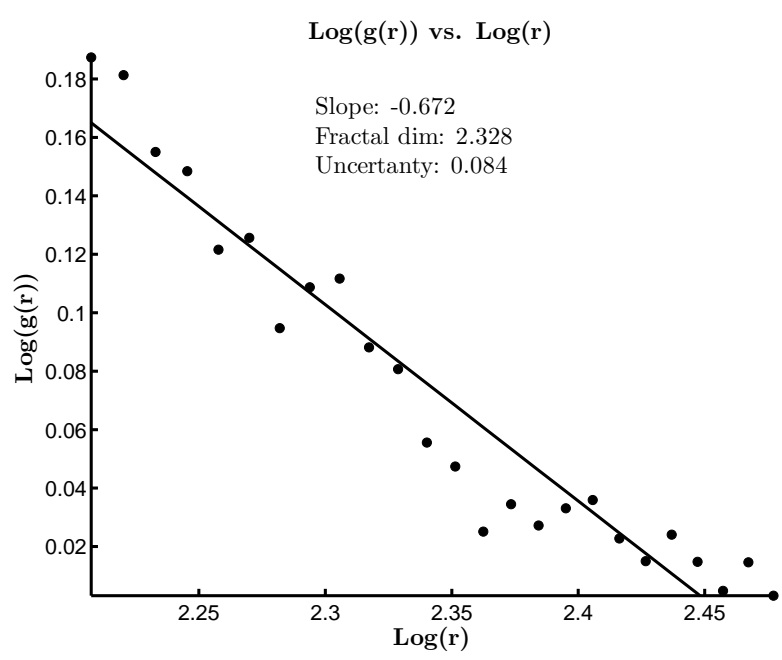

(b)

Figure 4.8: (a) Pair Distribution Function, (b) fractal dimension for hybrid aerogel, $\rho=0.45 \frac{\mathrm{g}}{\mathrm{cm}^{3}}, 0.1 \mathrm{~mol} \%$ $\mathrm{CH}_{3}$ bonded to $\mathrm{Si}$.

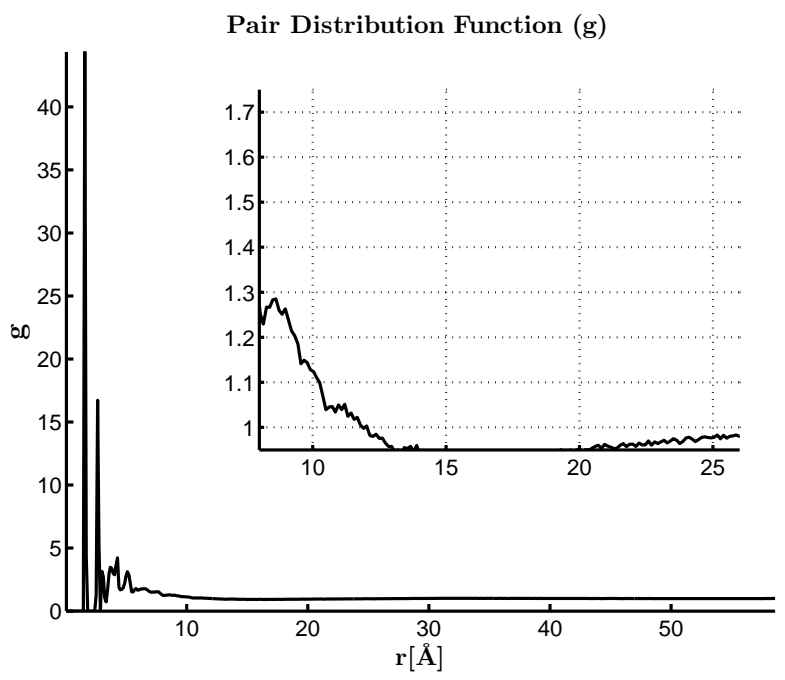

(a)

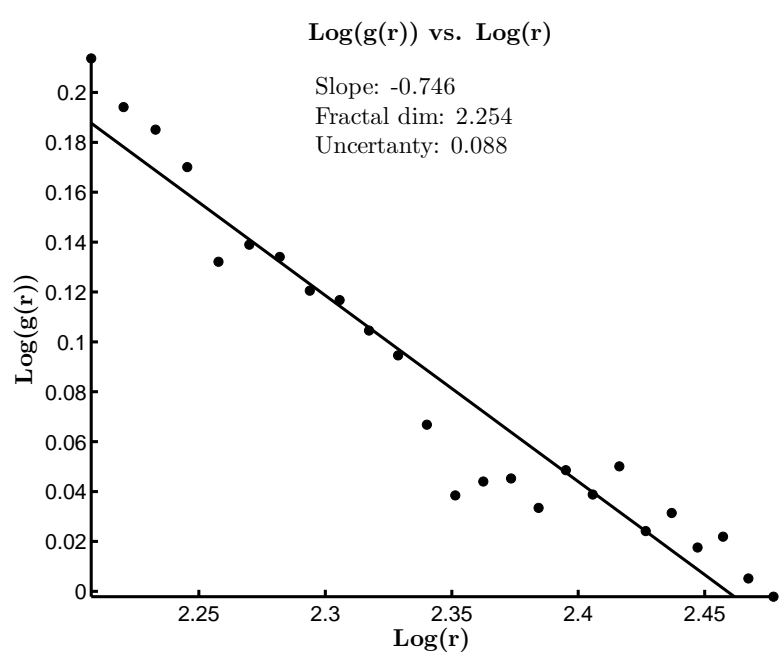

(b)

Figure 4.9: (a) Pair Distribution Function, (b) fractal dimension for hybrid aerogel, $\rho=0.45 \frac{\mathrm{g}}{\mathrm{cm}^{3}}, 0.1 \mathrm{~mol} \%$ $\mathrm{CH}_{3}$ bonded to $\mathrm{O}$. 


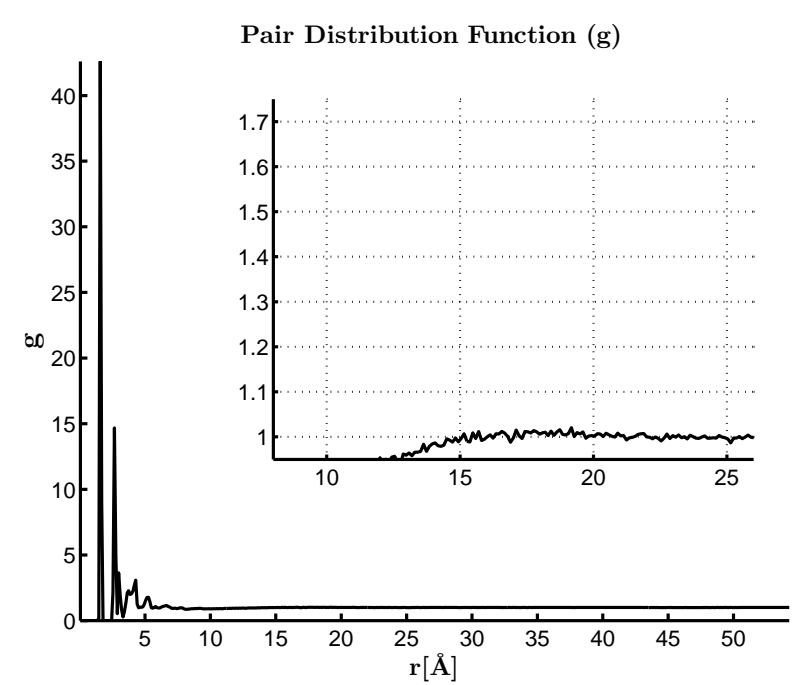

(a)

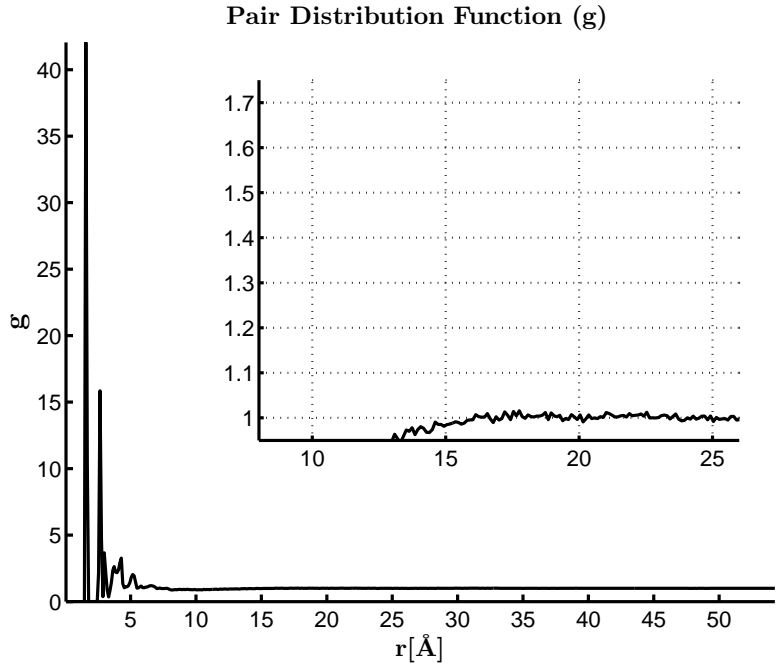

(b)

Figure 4.10: Pair Distribution Function hybrid aerogel, $\rho=0.45 \frac{g}{\mathrm{~cm}^{3}}, 0.4 \mathrm{~mol} \% \mathrm{CH}_{3}$ content. (a) $\mathrm{CH}_{3}$ bonded to $\mathrm{Si}$, (b) $\mathrm{CH}_{3}$ bonded to $\mathrm{O}$

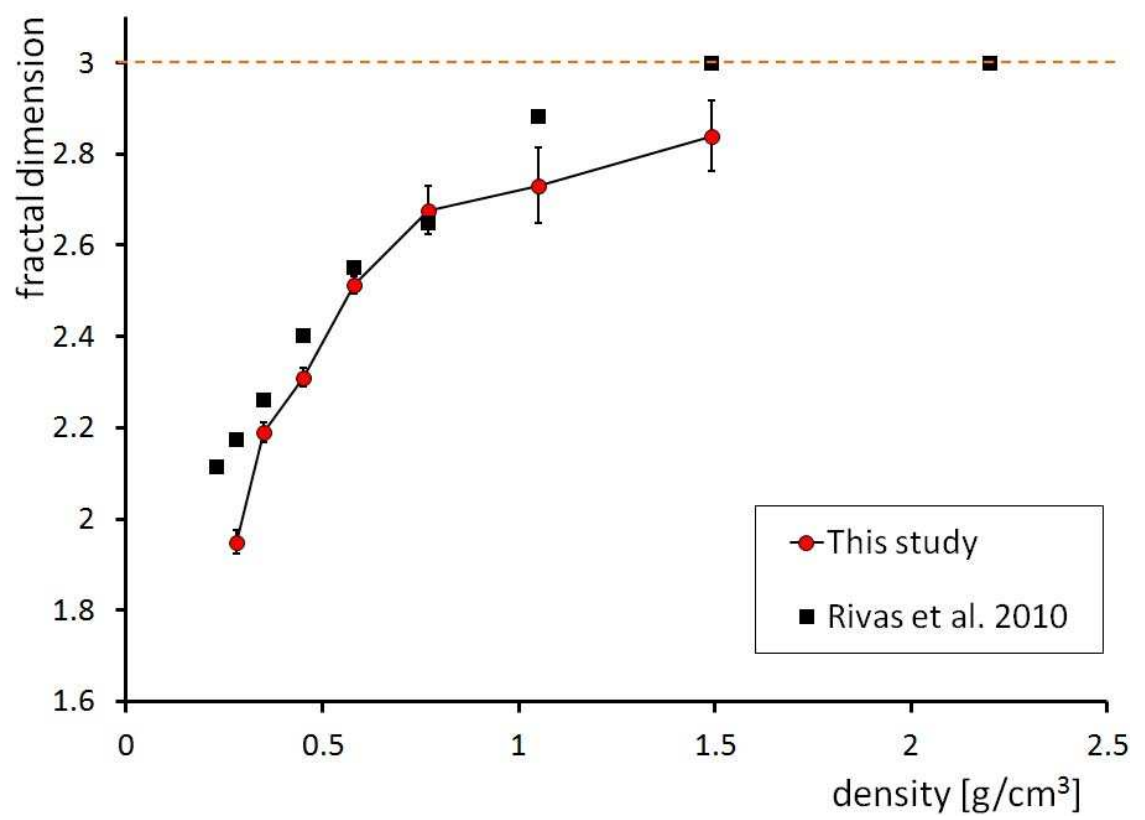

Figure 4.11: Fractal dimension vs. density plot for silica aerogels and xerogels. Rivas et al. 2010 [64] 


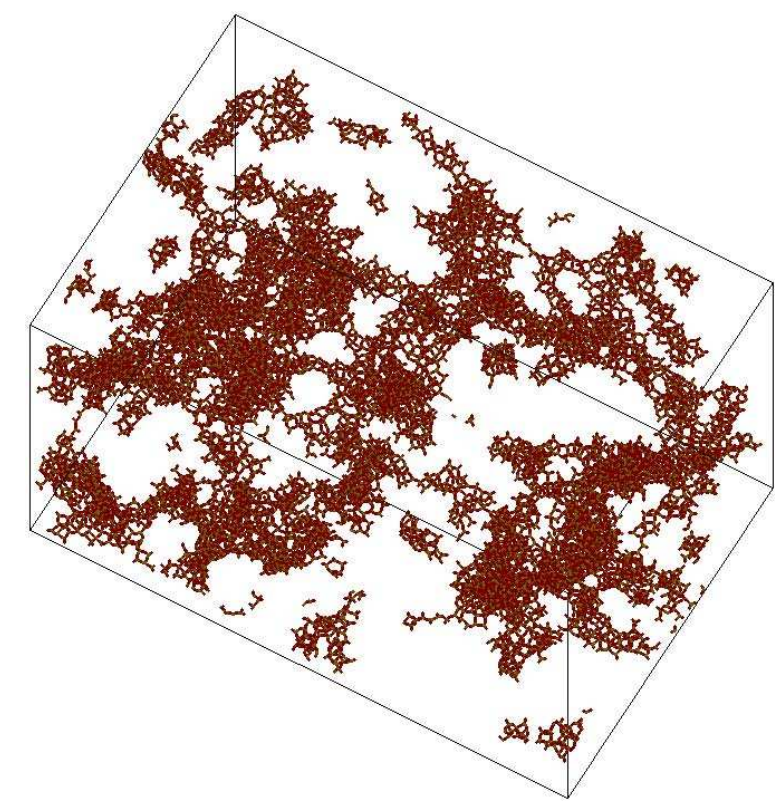

Figure 4.12: Silica aerogel sample, density $0.28 \frac{\mathrm{g}}{\mathrm{cm}^{3}}$, after tension test 62.

also different. Both procedures can be used to generate samples that are surrogate models of aerogel and xerogel materials.

\subsection{Mechanical Characterization of $\mathrm{CH}_{3}$-Added Systems}

Tension tests for all the samples were simulated using the same conditions applied for the silica aerogel and xerogel samples as in 64] (also see section 3.5.3), i.e. stretching by scaling the atomic positions at a strain rate of $0.004 \mathrm{ps}^{-1}$ until the strain reached $50 \%$, temperature set at $0 \mathrm{~K}$ and controlled using Langevin dynamics with a damping factor, $\gamma$, of $1 \mathrm{ps}^{-1}$. Samples of silica and hybrid aerogels, density $0.28 \frac{\mathrm{g}}{\mathrm{cm}^{3}}$, with $0 \mathrm{~mol} \%, 0.1 \mathrm{~mol} \%$ and $0.4 \mathrm{~mol} \% \mathrm{CH}_{3}$ content, after the tension test are presented in Figs. 4.12-4.14, Figures corresponding to the additional samples are presented in appendix $\mathrm{A}$.

The stress vs. strain plots for a sample of density $0.77 \frac{\mathrm{g}}{\mathrm{cm}^{3}}$, having $0 \mathrm{~mol} \%, 0.1 \mathrm{~mol} \%$ and 0.4 mol\% $\mathrm{CH}_{3}$ content, are illustrated in 4.15. Stress vs. strain plots for all the additional samples are included in appendix C.

From Fig. 4.15(b) and (c) it is concluded that there are no major differences between the stress-strain responses of samples containing molecules of $\mathrm{CH}_{3}$ bonded to $\mathrm{Si}$ and $\mathrm{CH}_{3}$ bonded to O. The plot for the samples with $0.4 \mathrm{~mol} \% \mathrm{CH}_{3}$ bonded to Si content is very similar to that of the sample with $0.4 \mathrm{~mol} \% \mathrm{CH}_{3}$ bonded to O. Likewise, although to a lesser extend, it happens with the samples containing $0.1 \mathrm{~mol}^{\circ} \mathrm{CH}_{3}$. 

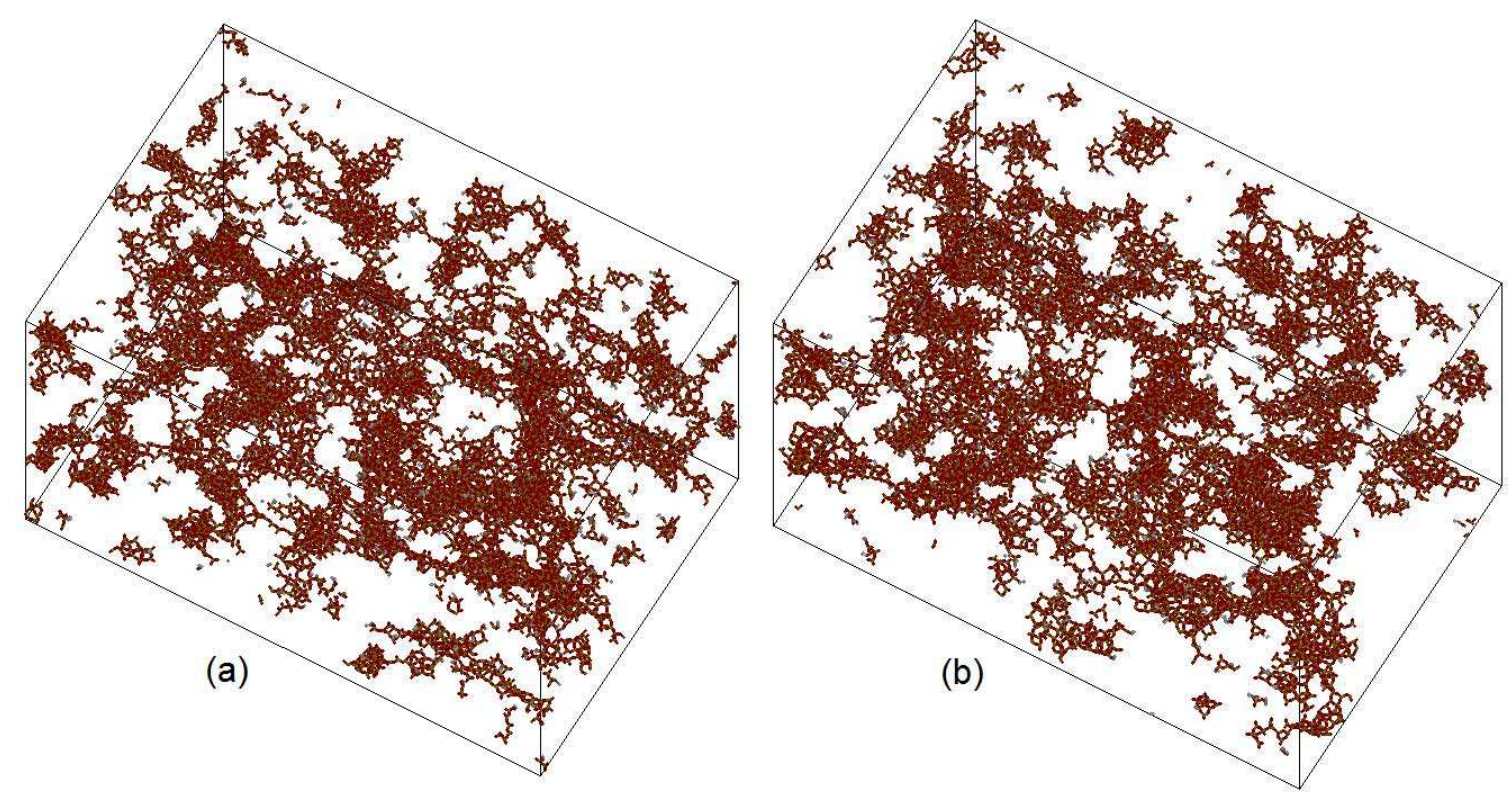

Figure 4.13: Aerogel sample with $0.1 \mathrm{~mol} \% \mathrm{CH}_{3}$ content, density $0.28 \frac{\mathrm{g}}{\mathrm{cm}^{3}}$, after tension test 62. (a) $\mathrm{CH}_{3}$ bonded to $\mathrm{Si}$, (b) $\mathrm{CH}_{3}$ bonded to $\mathrm{O}$.
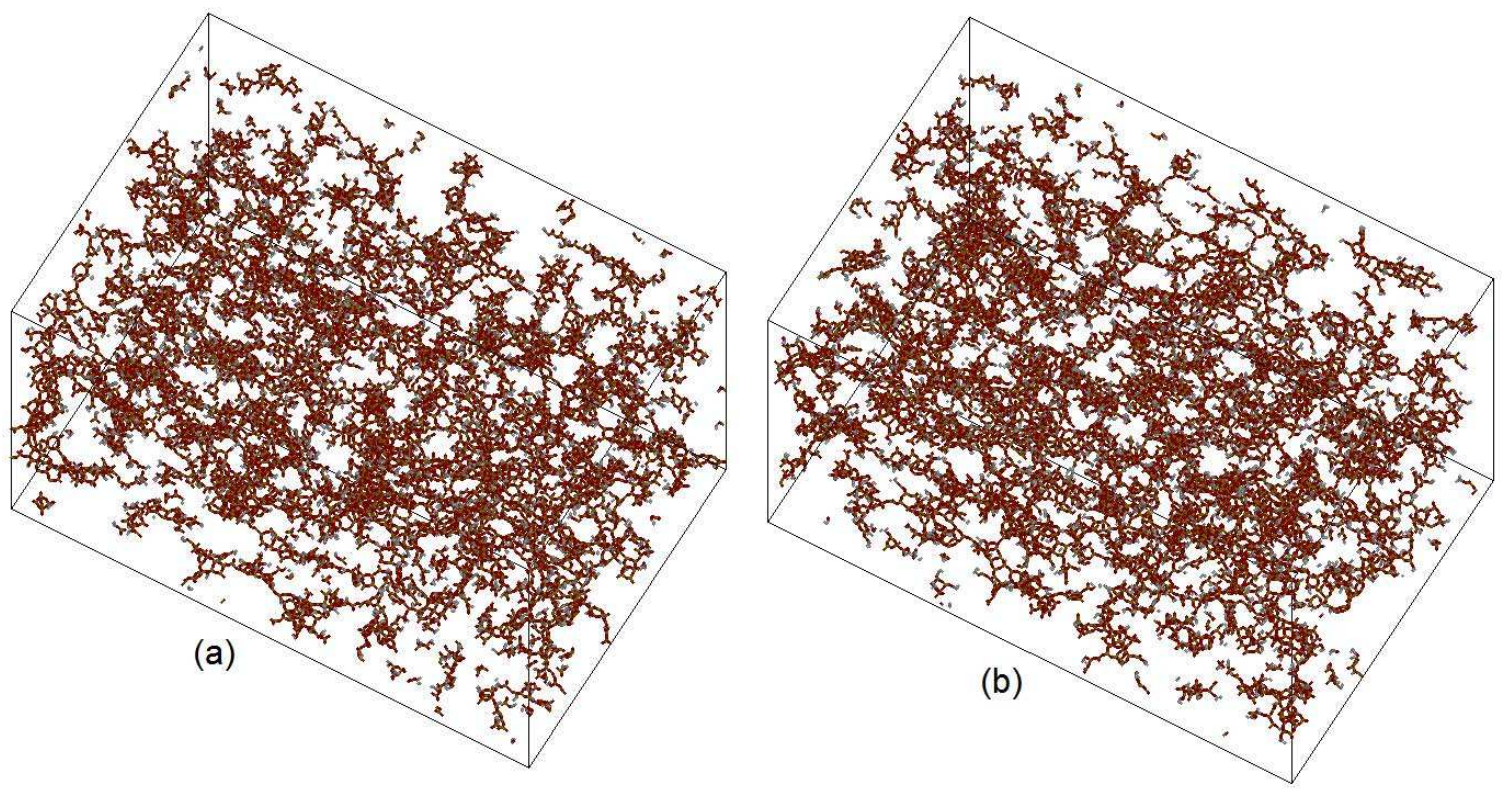

Figure 4.14: Aerogel sample with $0.4 \mathrm{~mol} \% \mathrm{CH}_{3}$ content, density $0.28 \frac{\mathrm{g}}{\mathrm{cm}^{3}}$, after tension test 62. (a) $\mathrm{CH}_{3}$ bonded to $\mathrm{Si}$, (b) $\mathrm{CH}_{3}$ bonded to $\mathrm{O}$. 
In Fig. 4.15(d) it is seen that the strength of the sample is not affected by the addition of $\mathrm{CH}_{3}$ in the structure. Since the strength of the samples is related to their hardness, this result is in agreement with experimental findings in which the hardness of hybrid aerogel was not influenced by the amount of organic content [18]. Contrary to the strength, the stiffness of the samples is highly affected by the amount of $\mathrm{CH}_{3}$ in the structure. Hybrid samples developed lower stress levels than silica samples at the same strain, therefore they can withstand larger strain without breaking.

The toughness of a material is defined as the amount of strain energy per unit of volume that it can absorb before breaking. It can be estimated from the results of a tension test as

$$
u=\int_{\epsilon=0}^{\epsilon_{f}} \sigma * d \epsilon
$$

with $\sigma$ being the stress, $\epsilon$ the strain, $\epsilon_{f}$ the strain at the breaking point of the sample. $u$ is equivalent to the area under the stress vs. strain plot. Since several of the samples used for this study did not break completely after they were stretched $50 \%$ of their original length, the estimation of the toughness was done integrating the stress-strain plot between the limits $\epsilon=0$ and $\epsilon=0.50$. The values obtained this way do not represent the exact toughness of the samples; but they are an useful tool to compare the different samples.

In Fig. 4.15(d) it is seen that the addition of $\mathrm{CH}_{3}$ into the silica structure dramatically improved the toughness of the samples. In Fig. 4.16 the toughness increase due to the addition of $0.4 \mathrm{~mol} \%$ $\mathrm{CH}_{3}$ into to the silica structure is presented. The presence of $\mathrm{CH}_{3}$ in the structure of silica caused a toughness increase of more than twice the value for pure silica. The effect is more evident for denser, hence stiffer, samples.

The improvement of the toughness of silica aerogels and xerogels samples caused by the addition of $\mathrm{CH}_{3}$ is due to the structural transformation generated by the $\mathrm{CH}_{3}$ molecules. $\mathrm{CH}_{3}$ molecules hinder the formation of $\mathrm{O}-\mathrm{Si}-\mathrm{O}$ bonds by either bonding to the $\mathrm{Si}$ or $\mathrm{O}$ atoms, the overall effect is the same. $\mathrm{Si}$ and $\mathrm{O}$ atoms are less cross-linked, hence $\mathrm{Si}-\mathrm{O}$ bonds can stretch and rotate more easily than in pure silica structures.

By hindering the creation of $\mathrm{O}-\mathrm{Si}-\mathrm{O}$ bonds, the presence of $\mathrm{CH}_{3}$ in the structure facilitates the formation of smaller clusters and smaller pores than in structures without $\mathrm{CH}_{3}$. The pores are also more uniformly distributed across the structure. Thanks to those structural features, when samples are stretched the breaking of bonds and chains of atoms has a less dramatic effect than it has in pure silica structures, which are formed by larger clusters. The strength of the samples is not affected by the addition of $\mathrm{CH}_{3}$ because the structures carrying load are formed by $\mathrm{Si}-\mathrm{O}$ bonds only.

The relationships between elastic modulus and density, and between strength and density are illustrated in Figs. 4.17- 4.22, The relationships are described by power laws, which agrees with 


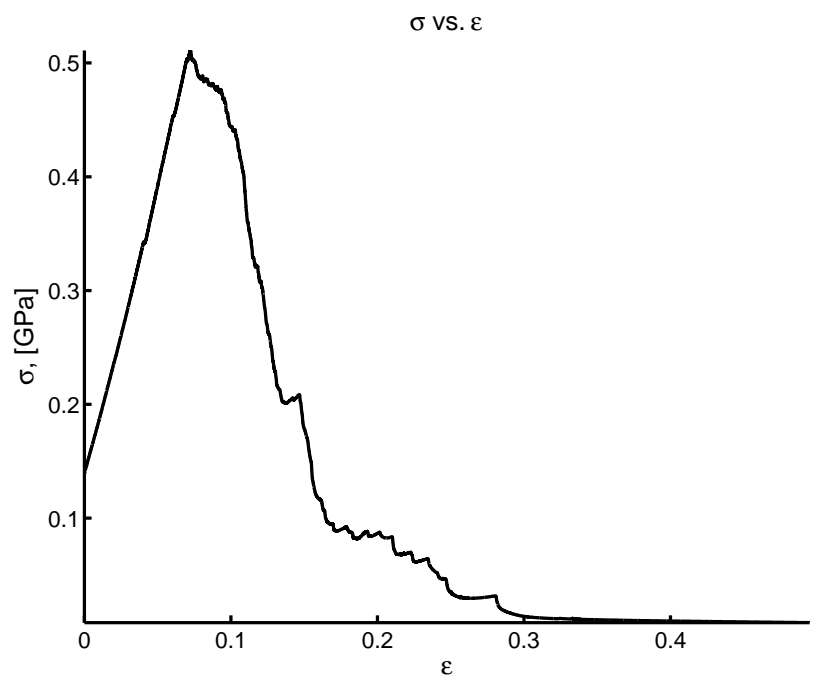

(a)

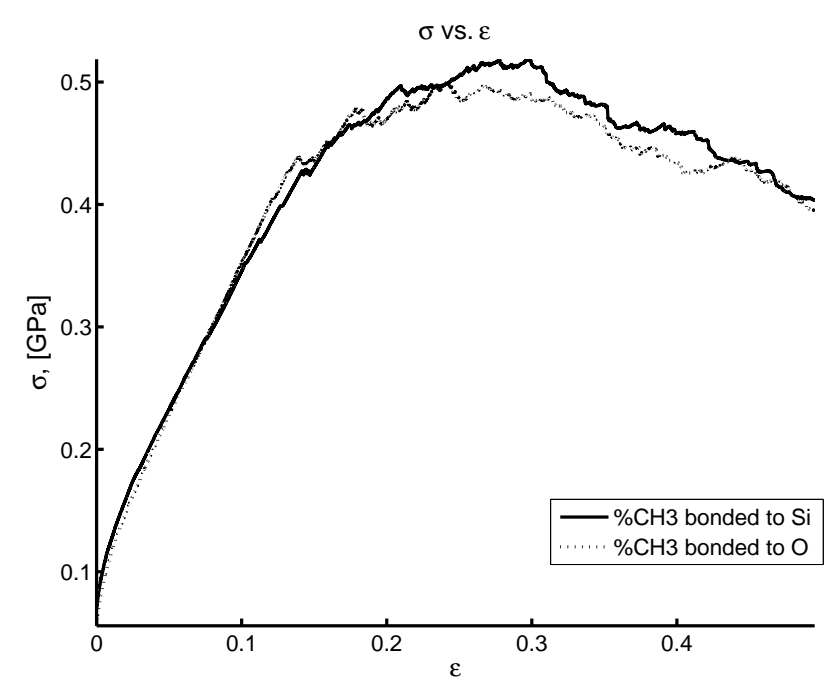

(c)

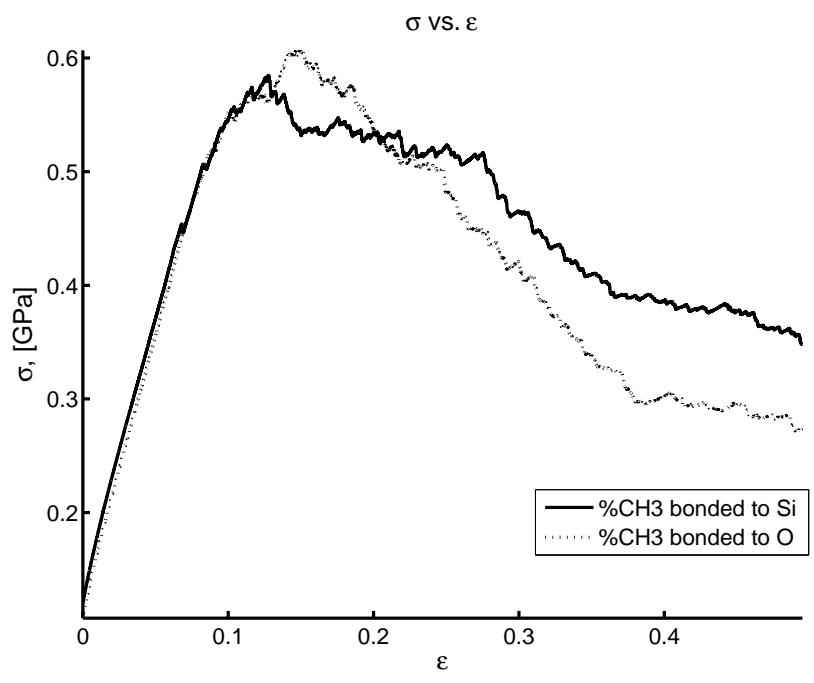

(b)

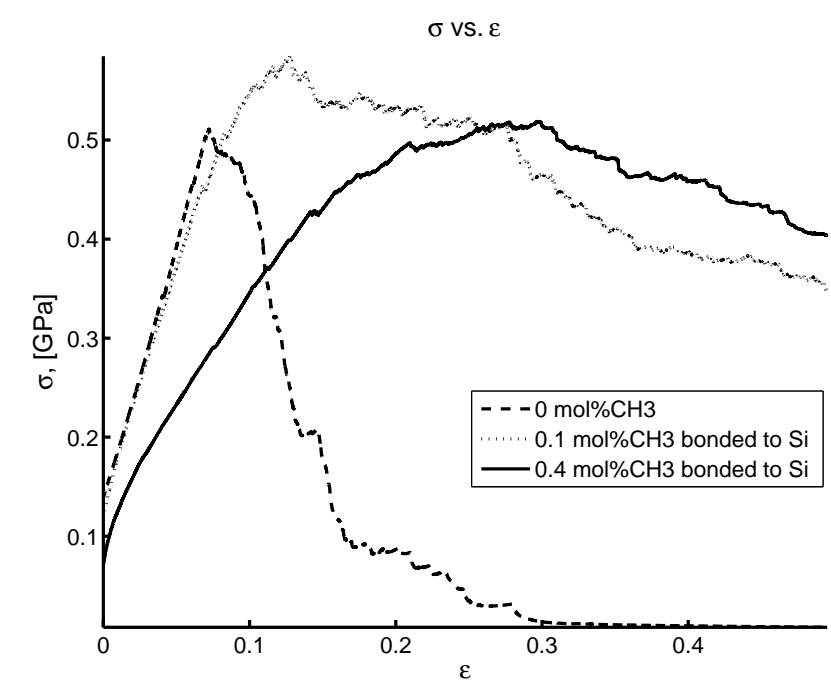

(d)

Figure 4.15: Stress vs. Strain plot for silica and hybrid aerogel, density $0.77 \frac{\mathrm{g}}{\mathrm{cm}^{3}}$. (a) $0 \mathrm{~mol} \% \mathrm{CH}_{3}$, (b) 0.1 $\mathrm{mol} \% \mathrm{CH}_{3}$, (c) $0.4 \mathrm{~mol} \% \mathrm{CH}_{3}$, (d) $0 \mathrm{~mol} \%$, $0.1 \mathrm{~mol} \%$, and $0.4 \mathrm{~mol} \% \mathrm{CH}_{3}$ 


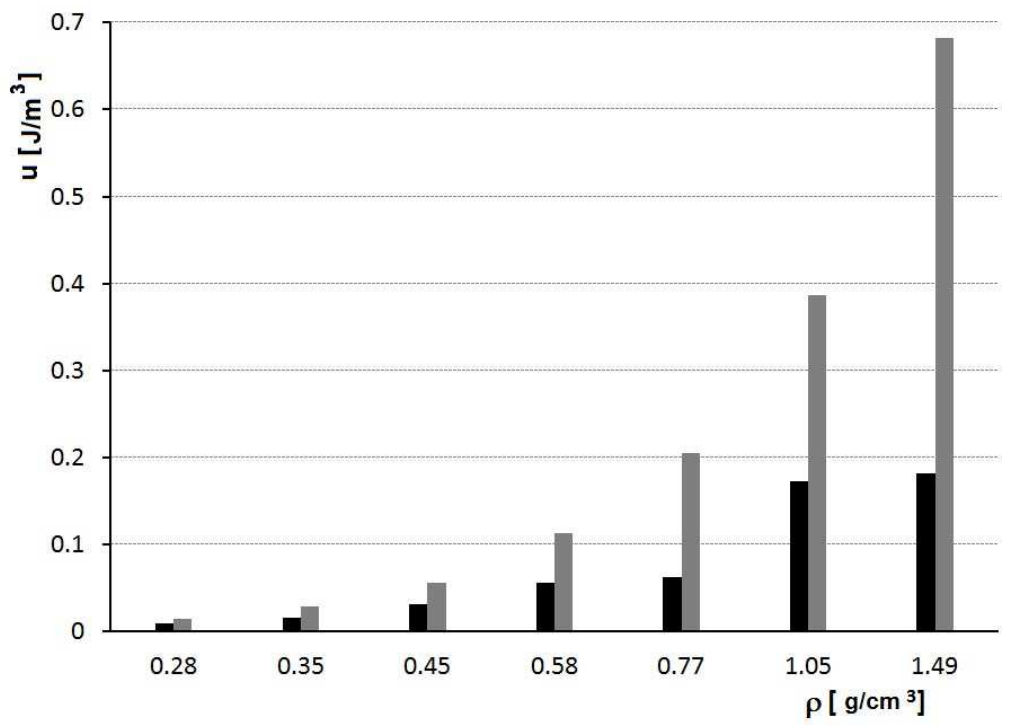

Figure 4.16: Toughness vs. density for silica and hybrid aerogels and xerogels. Silica: black bars, hybrid: gray bars. Toughness of hybrid samples calculated averaging the values for $\mathrm{CH}_{3}$ bonded to $\mathrm{Si}$ and $\mathrm{CH}_{3}$ bonded to O samples.

experimental data [10, 8] and numerical data [50, 64] for silica aerogels and xerogels. For pure silica samples, the elastic modulus vs. density, and strength vs. density relationships are characterized by exponents of $3.11 \pm 0.34$ and $2.65 \pm 0.31$ respectively. The values are similar to those reported in [64] (also see section 3.5.3) which further confirms that the procedure used to create the samples for this study leads to the formation of surrogate models of aerogel and xerogel materials.

The addition of $\mathrm{CH}_{3}$ to the silica structure causes a reduction of the exponent of the relationship between elastic modulus and density, which is $3.11 \pm 0.34$ for samples with 0 mol\% $\mathrm{CH}_{3}, 2.26 \pm$ 0.11 and $2.30 \pm 0.12$ for samples with $0.1 \mathrm{~mol}^{2} \mathrm{CH}_{3}$ bonded to Si and $\mathrm{O}$ atoms, respectively, and $1.99 \pm 0.19$ and $2.12 \pm 0.20$ for samples with $0.4 \mathrm{~mol} \% \mathrm{CH}_{3}$ bonded to Si and $\mathrm{O}$ atoms, respectively. The reduction of the exponent is consistent with the finding that $\mathrm{CH}_{3}$ addition effectively reduces the stiffness and increases the toughness of the structure, this effect being more significant in denser samples (see Fig. 4.16). The exponent of the strength vs. density relationship is $2.65 \pm 0.31$ for samples with $0 \mathrm{~mol} \% \mathrm{CH}_{3}, 2.12 \pm 0.22$ and $2.22 \pm 0.30$ for samples containing $0.1 \mathrm{~mol} \%$ of $\mathrm{CH}_{3}$ bonded to $\mathrm{Si}$ and $\mathrm{O}$ atoms respectively, and $2.23 \pm 0.22$ and $2.11 \pm 0.35$ for samples containing $0.4 \mathrm{~mol} \% \mathrm{CH}_{3}$ bonded to $\mathrm{Si}$ and $\mathrm{O}$ atoms respectively. The exponents for those relationships are virtually the same because the addition of $\mathrm{CH}_{3}$ does not affect the strength of the sample. Finally, it is worth noticing that the exponents of the relationships, and the magnitude of the values for elastic modulus and strength are very similar for samples having $\mathrm{CH}_{3}$ bonded to $\mathrm{Si}$ and bonded to $\mathrm{O}$ atoms. This provides additional support to the notion that one of the main effects of adding $\mathrm{CH}_{3}$ to the system is hindering the formation of $\mathrm{O}-\mathrm{Si}-\mathrm{O}$ bonds, and the overall results are the same independent of the type of atoms to which the $\mathrm{CH}_{3}$ molecules bond. 


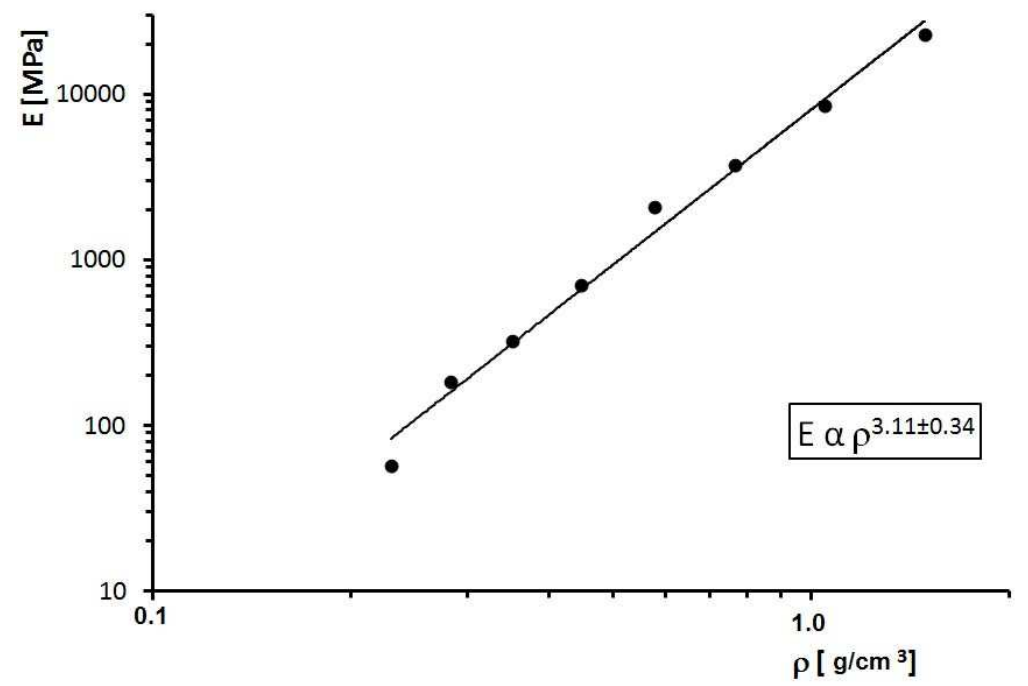

Figure 4.17: Elastic modulus vs. density plot for silica aerogels and xerogels

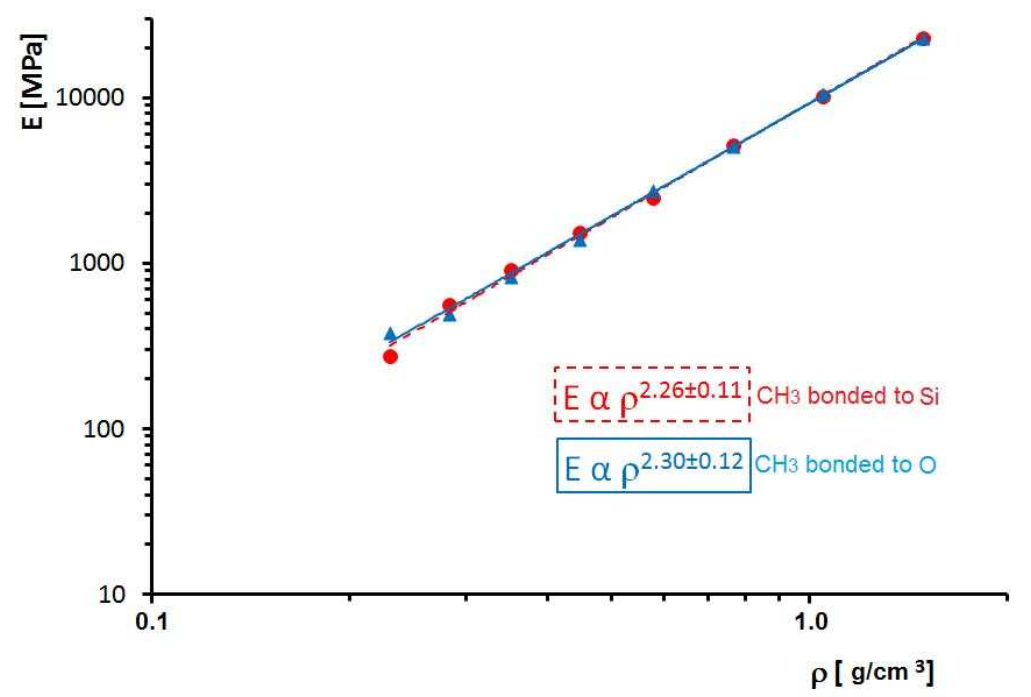

Figure 4.18: Elastic modulus vs. density plot for hybrid aerogels and xerogels, $0.1 \mathrm{~mol} \% \mathrm{CH}_{3}$ content. $\mathrm{CH}_{3}$ bonded to $\mathrm{O}$ : triangular marker and solid line, $\mathrm{CH}_{3}$ bonded to $\mathrm{Si}$ : circular marker and dotted line 


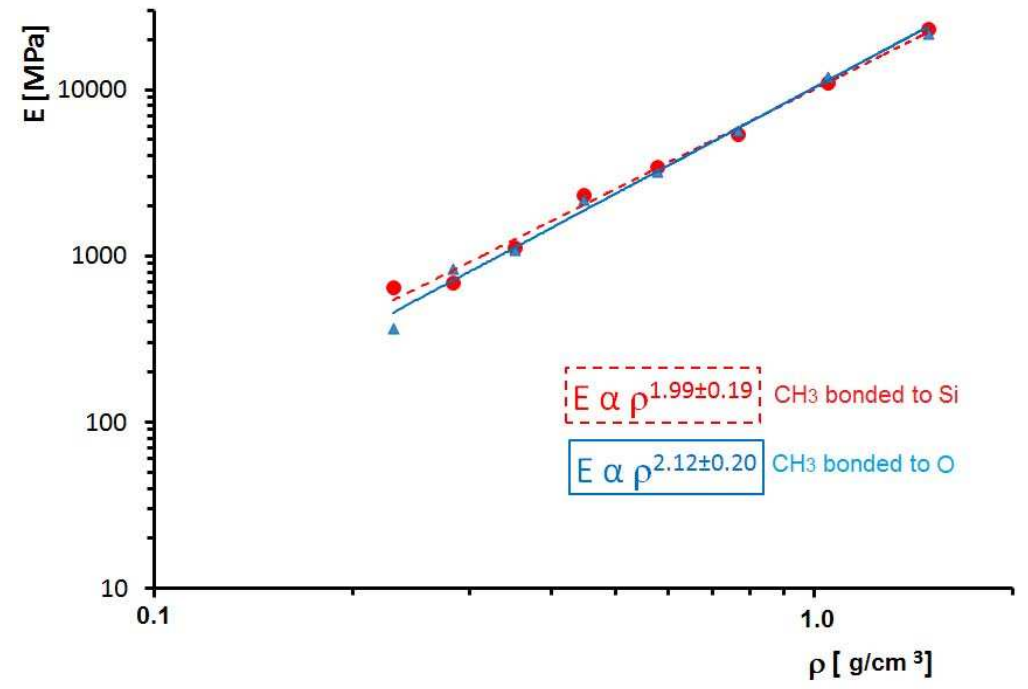

Figure 4.19: Elastic modulus vs. density plot for hybrid aerogels and xerogels, $0.4 \mathrm{~mol} \% \mathrm{CH}_{3}$ content. $\mathrm{CH}_{3}$ bonded to O: triangular marker and solid line, $\mathrm{CH}_{3}$ bonded to $\mathrm{Si}$ : circular marker and dotted line

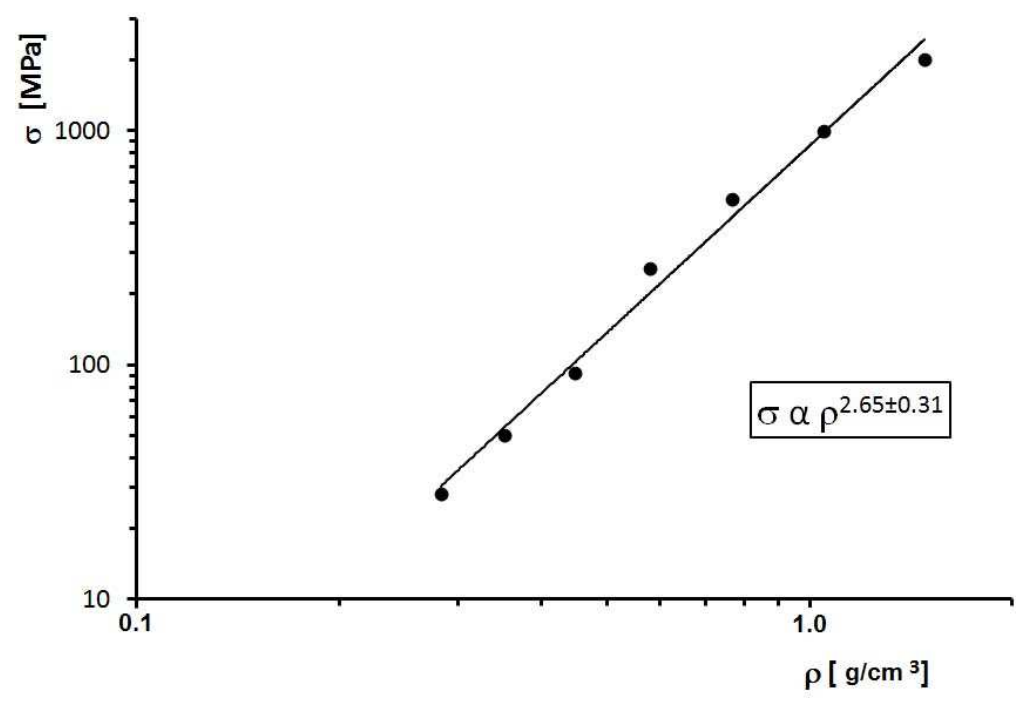

Figure 4.20: Strength vs. density plot for silica aerogels and xerogels 


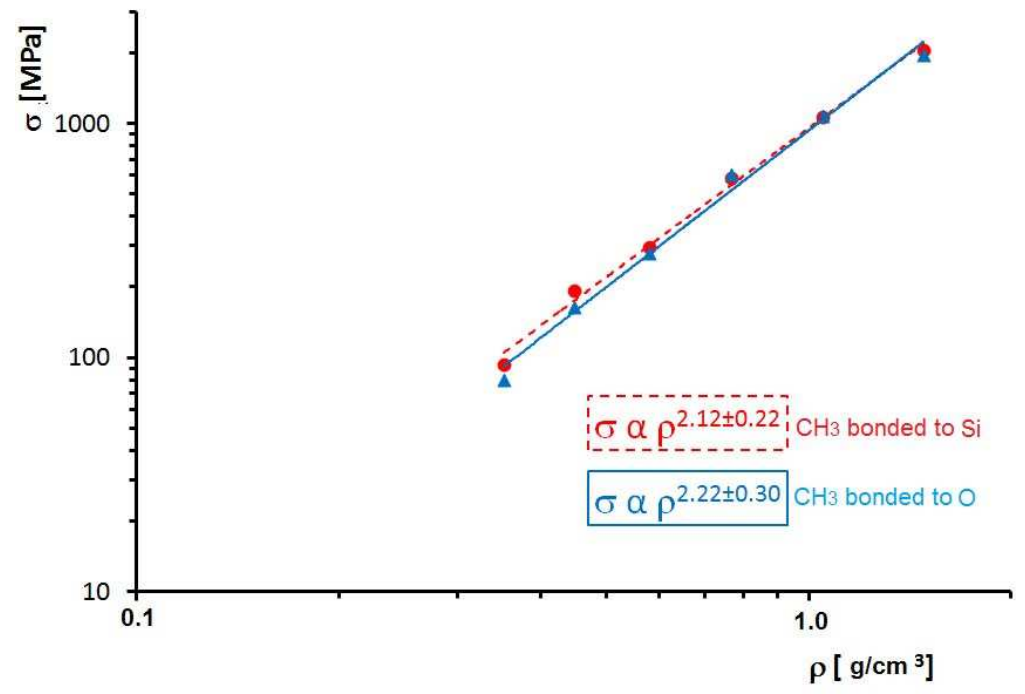

Figure 4.21: Strength vs. density plot for hybrid aerogels and xerogels, $0.1 \mathrm{~mol} \% \mathrm{CH}_{3}$ content. $\mathrm{CH}_{3}$ bonded to O: triangular marker and solid line, $\mathrm{CH}_{3}$ bonded to Si: circular marker and dotted line

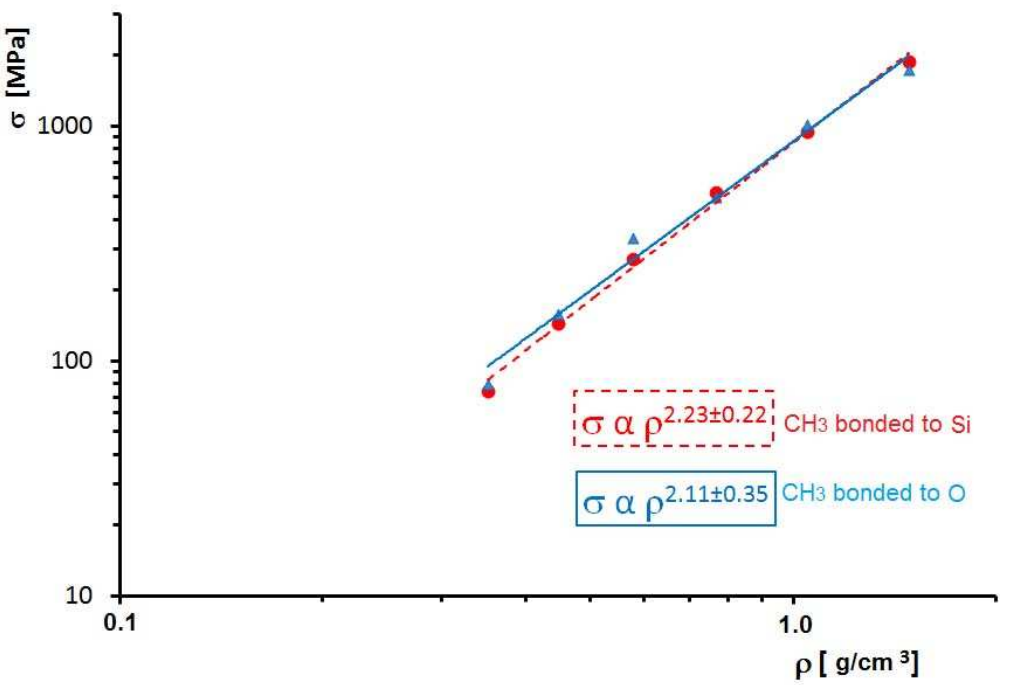

Figure 4.22: Strength vs. density plot for hybrid aerogels and xerogels, $0.4 \mathrm{~mol} \% \mathrm{CH}_{3}$ content. $\mathrm{CH}_{3}$ bonded to O: triangular marker and solid line, $\mathrm{CH}_{3}$ bonded to $\mathrm{Si}$ : circular marker and dotted line 


\section{Chapter 5}

\section{Conclusions}

Computational samples of porous silica were produced using two different preparation methods. Both methods consisted of expanding the atomic positions of silica samples, crystalline or glass, inside a simulation box. The volume of the simulation box was selected to guarantee that the porous samples have a specified density. In both preparation methods a thermal treatment followed the expansion of the initial sample. The thermal treatment consisted of heating up the samples by increasing the speed of the atoms, dwelling at certain temperatures, and cooling them down by reducing the speed of the atoms. When the samples are at high temperature, the atoms move at high speed and can travel over longer distances in the simulation box. When the samples are cooled down the atoms are locked in the atomic positions they are, and the structure is set. The preparation procedures differ in the details of the thermal treatment. The samples were cubic having densities varying between 0.23 and $2.2 \frac{\mathrm{g}}{\mathrm{cm}^{3}}$, and size varying from 71 to $151 \AA$.

The structural and mechanical properties of the porous silica samples, produced using both preparation methods, were characterized using the Pair Distribution Function, and simulating uniaxial tension tests. The structural characterization showed that the samples display fractal features in the intermediate-range order (9 to $30 \AA$ ). The fractal dimension of the samples, and the relationship between it and the density of the samples are in agreement with experimental and numerical data for silica aerogels and xerogels (see Figs. 3.21 and 4.11). This validates the procedures implemented in this research as methods to create computational surrogates of silica aerogels and xerogels. 
Further validation was provided by the mechanical characterization of the computational samples. The elastic modulus of the computational samples was found to be in agreement with experimental data. The elastic modulus vs. density relationships for the computational samples produced by both methods follow a power law characterized by exponents of $3.11 \pm 0.21$ and $3.11 \pm 0.34$ (see Figs. 3.24 and 4.17). Experimental samples have also shown that there is a power law relationship between elastic modulus and density characterized by an exponent of 3.2 $\pm 0.2[10,8]$. The strength of the computational samples also relates to the density following a power law relationship (see Figs. 3.25 and 4.20), as has been reported for silica and hybrid aerogels [10, 8]. The results of this research show that the exponents of the relationships are $2.53 \pm$ 0.15 , and $2.65 \pm 0.31$, the experimental value of the exponent is reported to be between 2.1 and 2.8.

Samples of hybrid aerogels and xerogels were produced by adding $0.1 \mathrm{~mol} \%$ and $0.4 \mathrm{~mol} \%$ of $\mathrm{CH}_{3}$ molecules to the silica structure. The $\mathrm{CH}_{3}$ molecules were bonded to $\mathrm{Si}$ or $\mathrm{O}$ atoms. The structural characterization of the samples showed that the extension of fractal region found in silica aerogels is reduced, and eventually eliminated by the addition of $\mathrm{CH}_{3}$ to the samples (see Figs. 4.7- 4.10). The addition of $\mathrm{CH}_{3}$ caused reduction of the cluster and the pore sizes in the sample, generating a more uniform pore distribution. Those findings have also been reported from experimental characterization of hybrid samples [18].

The addition of $\mathrm{CH}_{3}$ reduced the stiffness of the structures without affecting their strength (see Fig. 4.15). This caused a dramatic improvement of the toughness of the material, it increased more than twice when $0.4 \mathrm{~mol} \%$ of $\mathrm{CH}_{3}$ was added (see Fig. 4.16). The relationships between elastic modulus and density, and between strength and density are described by power laws, as is the case for pure silica samples. The exponents of the elastic modulus vs. density relationships were $2.30 \pm 0.12$ and $2.12 \pm 0.20$ for samples containing $0.1 \mathrm{~mol} \%$ and $0.4 \mathrm{~mol} \%$ of $\mathrm{CH}_{3}$ bonded to $\mathrm{O}$ atoms, respectively (see Figs. 4.18 and 4.19). And they were $2.26 \pm 0.11$ and $1.99 \pm 0.19$ for samples containing $0.1 \mathrm{~mol} \%$ and $0.4 \mathrm{~mol} \%$ of $\mathrm{CH}_{3}$ bonded to Si atoms, respectively (see Figs. 4.18 and 4.19). The reduction of the exponent with the increase of the $\mathrm{CH}_{3}$ content is consistent with the finding that the stiffness of denser samples is more affected by the addition of $\mathrm{CH}_{3}$. For the strength vs. density relationships the exponents were $2.22 \pm 0.30$ and $2.11 \pm 0.35$ for samples 
containing $0.1 \mathrm{~mol} \%$ and $0.4 \mathrm{~mol} \%$ of $\mathrm{CH}_{3}$ bonded to $\mathrm{O}$ atoms, respectively (see Figs. 4.21 and 4.22). And they were $2.12 \pm 0.22$ and $2.23 \pm 0.22$ for samples containing $0.1 \mathrm{~mol} \%$ and $0.4 \mathrm{~mol} \%$ of $\mathrm{CH}_{3}$ bonded to $\mathrm{Si}$ atoms, respectively (see Figs. 4.21 and 4.22 ). The exponents are very similar to those found for silica samples, further confirming that the addition of $\mathrm{CH}_{3}$ does not change the strength of the material.

Samples containing $\mathrm{CH}_{3}$ bonded to $\mathrm{Si}$ atoms have similar structural and mechanical properties than samples containing $\mathrm{CH}_{3}$ bonded to $\mathrm{O}$ atoms. This supports the notion that one of the main effects of adding $\mathrm{CH}_{3}$ to the system is hindering the formation of $\mathrm{O}-\mathrm{Si}-\mathrm{O}$ bonds, causing the formation of smaller clusters and more open structures independently of the type of atoms to which the $\mathrm{CH}_{3}$ molecules bond.

The results of this research provide an insight into the reasons why the addition of $\mathrm{CH}_{3}$ improves the toughness of silica aerogels and xerogels. It is due to structural transformations forced by the presence of $\mathrm{CH}_{3}$ molecules blocking the formation of $\mathrm{Si}-\mathrm{O}$ bonds. $\mathrm{CH}_{3}$-added silica aerogels and xerogels have smaller clusters and more open pore porous structure which generates samples with larger surface area, an important property for materials used in catalysis.

Other applications that could benefit from the use of $\mathrm{CH}_{3}$-added silica aerogels and xerogels are thermal and acoustic insulation. Those applications use the fact that the structure of aerogels and xerogels provide a cumbersome path for heat conduction and mechanical energy transfer. In $\mathrm{CH}_{3}$-added silica aerogels and xerogels smaller clusters and pores would provide an even more unsuitable path for energy transfer. 


\section{Bibliography}

[1] G. C. Ruben et al., "Imaging aerogels at the molecular level," Journal of Materials Science, vol. 27, pp. 4341-4349, 1992.

[2] Technical Insights Inc., Aerogels and xerogels: Growth and opportunities for the early $21^{\text {st }}$ century (Emerging technologies series). Technical Insights, 1996.

[3] H. D. Gesser and P. C. Goswami, "Aerogels and related porous materials," Chemical Reviews, vol. 89, no. 4, pp. 765-788, 1989.

[4] J. Fricke and T. Tillotson, "Aerogels: production, characterization and applications," Thin Solid Films, vol. 297, pp. 213-223, 1997.

[5] T. Woignier and J. Phalippou, "Mechanical strength of silica aerogels," Journal of NonCrystalline Solids, vol. 100, pp. 404-408, 1988.

[6] T. Burger and J. Fricke, "Aerogels: production, modification and applications," Berichte der Bunsengesellschaft für Physikalische Chemie, vol. 102, no. 11, pp. 1523-1528, 1998.

[7] R. K. Iler, The Chemistry of Silica: Solubility, Polymerization, Colloid and Surface Properties and Biochemistry of Silica. Wiley-Interscience, 1979.

[8] T. Woignier, J. Reynes, A. Hafidi Alaoui, I. Beurroies, and J. Phalippou, "Different kinds of structures in aerogels: relationship with mechanical properties," Journal of Non-Crystalline Solids, vol. 241, pp. 45-52, 1998.

[9] J. Fricke and G. Reichernauer, "Structural investigations of $\mathrm{SiO}_{2}$ aerogels," Journal of NonCrystalline Solids, vol. 95-96, pp. 1135-1142, 1987. 
[10] T. Woignier et al., "Elastic properties of silica aerogels," Journal of Non-Crystalline Solids, vol. 95-96, pp. 1197-1202, 1987.

[11] T. Woignier, J. Phalippou, R. Vacher, J. Pelous, and E. Courtens, "Different kinds of fractal structures in silica aerogels," Journal of Non-Crystalline Solids, vol. 121, pp. 198-201, 1990.

[12] J. Groß and J. Fricke, "Scaling of elastic properties in highly porous nanostructured aerogels," Nanostructured Materials, vol. 6, pp. 905-908, 1995.

[13] N. Hüsing and U. Schubert, "Aerogels - airy materials: Chemistry, structure, and properties," Angewandte Chemie International Edition, vol. 37, pp. 22-45, 1998.

[14] K. Kanamori, M. Aizawa, K. Nakanishi, and T. Hanada, "Elastic organic-inorganic hybrid aerogels and xerogels," Journal of Sol-Gel Science and Technology, vol. 48, pp. 172-181, 2008.

[15] F. Schwertfeger, W. Glaubitt, and U. Schubert, "Hydrophobic aerogels from $\mathrm{Si}(\mathrm{OMe})_{4} / \mathrm{Me}(\mathrm{Si}(\mathrm{OMe}))_{3}$ mixtures," Journal of Non-Crystalline Solids, vol. 145, pp. 85-89, 1992.

[16] F. Schwertfeger, A. Emmerling, J. Gross, U. Schubert, and J. Fricke, "Organically modified silica aerogels," in Sol-Gel processing and applications (Y. Attia, ed.), pp. 85-89, Plenum Press, 1994.

[17] F. Schwertfeger, N. Hüsing, and U. Schubert, "Influence of the nature of organic groups on the properties of organically modified silica aerogels," Journal of Sol-Gel Science and Technology, vol. 2, pp. 103-108, 1994.

[18] L. Martin, J. O. Ossó, S. Ricart, A. Roig, O. Garcia, and R. Sastre, "Organo-modified silica aerogels and implications for material hydrophobicity and mechanical properties," Journal of Materials Chemistry, vol. 18, pp. 207-213, 2008.

[19] T. M. Tillotson, K. G. Foster, and J. G. Reynolds, "Structure and characterization of aerogel materials and oxidation products from the reaction of $\left(\mathrm{CH}_{3} \mathrm{O}\right)_{4} \mathrm{Si}$ and $\mathrm{RSi}\left(\mathrm{CH}_{3} \mathrm{O}\right)_{3}$," Journal of Non-Crystalline Solids, vol. 350, pp. 209-215, 2004. 
[20] N. de la Rosa-Fox, V. Morales-Flórez, J. A. Toledo-Fernández, M. Piñero, R. Medoza-Serna, and L. Esquivias, "Nanoindentation on hybrid organic/inorganic silica aerogels," Journal of the European Ceramic Society, vol. 27, pp. 3311-3316, 2007.

[21] M. Piñero, V. Morales-Flórez, N. de la Rosa-Fox, and L. Esquivias, "Propiedades mecánicas de aerogeles híbridos de silicé," Boletín de la Sociedad Española de Céramica y Vidrio, vol. 44, no. 5, pp. 291-293, 2005.

[22] G. Sutmann, "Molecular dynamics - vision and reality," in Computational nanoscience: Do it yourself! (J. Grotendorst, S. Blügel, and D. Marx, eds.), vol. 31, (Forschungszentrum, Jülich, Germany), pp. 159-194, John von Neumann Institut für Computing, Feb. 2006.

[23] M. P. Allen and D. J. Tildesley, Computer Simulation of Liquids. New York, NY, USA: Oxford University Press, 1989.

[24] M. P. Allen, "Introduction to molecular dynamics simulation," in Computational soft matter: From synthetic polymers to proteins (N. Attig, K. Binder, H. Grubmüller, and K. Kremer, eds.), vol. 23, (Gustav-Stresemann-Institut, Bonn, Germany), pp. 1-28, John von Neumann Institut für Computing, Feb. 2004.

[25] M. Griebel, S. Knapek, and G. Zumbusch, Numerical simulation in molecular dynamics: Numerics, algorithms, parallelization, applications. USA: Springer, 2007.

[26] D. Frenkel and B. Smit, Understanding molecular dynamics simulation, $2^{\text {nd }}$ edition. San Diego, CA, USA: Academic Press, 2002.

[27] J. P. Ryckaert, G. Ciccotti, and H. J. C. Berendsen, "Numerical integration of Cartesian equations of motion of a system with constraints: Molecular Dynamics of n-Alkanes," Journal of Computational Physics, vol. 23, pp. 327-341, 1977.

[28] J. P. Ryckaert, G. Arialdi, and S. Melchionna, "Molecular dynamics of polymers with explicit but frozen hydrogens," Molecular Physics, vol. 99, no. 3, pp. 155-165, 2001.

[29] D. Brown, J. H. R. Clarke, M. Okuda, and T. Yamazaki, "A domain decomposition parallel processing algorithm for molecular dynamics simulations of polymers," Computer Physics Communications, vol. 83, no. 1, pp. 1-13, 1994. 
[30] A. R. C. Raine, "Molecular dynamics simulation of proteins on an array of transputers," in Applications of Transputers 2. Proceedings of the Second International Conference on Applications of Transputers, (July 11 th $-13^{\text {th }} 1990$, Southampton, UK), 1990.

[31] X.-W. Wu and S.-S. Sung, "Constraint dynamics algorithm for simulation of semiflexible macromolecules," Journal of Computational Chemistry, vol. 19, no. 14, pp. 1555-15566, 1998.

[32] E. S. R. Gopal, Statistical Mechanics and Properties of Matter. UK: Ellis Horwood Ltd., 1974.

[33] H. Goldstein, Classical Mechanics, $2^{\text {nd }}$ edition. USA: Addison-Wesley, 1980.

[34] A. G. McLellan, "Virial theorem generalized," American Journal of Physics, vol. 42, pp. 239$243,1974$.

[35] M. Zhuo, "A new look at the atomic level virial stress: On continuum-molecular system equivalence," Proceedings of the Royal Society A, vol. 459, pp. 2347-2392, 2003.

[36] P. Vashishta, R. K. Kalia, J. P. Rino, and I. Ebbsjö, "Interaction potential for $\mathrm{SiO}_{2}$ : A molecular-dynamics study of structural correlations," Physical Review B, vol. 15, pp. 1219712209, 1990.

[37] A. C. Wright, "The comparison of molecular dynamics simulations with diffraction experiments," Journal of Non-Crystalline Solids, vol. 159, pp. 264-268, 1993.

[38] R. Vacher, T. Woignier, and J. Pelous, "Structure and self-similarity of silica aerogels," Physical Review B, vol. 37, no. 11, pp. 6500-6503, 1988.

[39] F. Devreux, J. P. Boilot, F. Chaput, and B. Sapoval, "NMR determination of the fractal dimension in silica aerogels," Physical Review Letters, vol. 65, no. 5, pp. 614-617, 1990.

[40] T. Vicsek, Fractal growth phenomena. Singapore: World Scientific, 1992.

[41] J. Kieffer and C. A. Angell, "Generation of fractal structures by negative pressure rupturing of $\mathrm{SiO}_{2}$ glass," Journal of Non-Crystalline Solids, vol. 106, pp. 336-342, 1988.

[42] M. Kallala, B. Jullien, and B. Cabane, "Crossover from gelation to precipitation," Journal de Physique II, vol. 2, pp. 7-25, 1992. 
[43] A. Emmerling and J. Fricke, "Scaling properties and structure of aerogels," Journal of Sol-Gel Science and Technology, vol. 8, pp. 781-788, 1997.

[44] T. Nakayama and K. Yahubo, "Dynamical properties of fractal networks: scaling, numerical simulations and physical realizations," Reviews of Modern Physics, vol. 66, no. 2, pp. 381-443, 1994.

[45] V. V. Zosimov and L. M. Lyamshev, "Fractals in wave processes," Physics-Uspekhi, vol. 38, no. 4, pp. 347-484, 1995.

[46] P. Vashishta, R. K. Kalia, A. Nakano, W. Li, and I. Ebbsjö, "Molecular dynamics methods and large-scale simulations of amorphous materials," in Amorphous insulators and semiconductors (M. F. Thorpe and M. I. Mitkova, eds.), vol. 23, pp. 151-213, Kluwer Academic Publishers, 1997. NATO ASI Series, 3 High Technology.

[47] A. Nakano, L. Bi, R. K. Kalia, and P. Vashishta, "Structural correlations in porous silica: Molecular dynamics simulation on a parallel computer," Physical Review Letters, vol. 71, no. 1, pp. 85-88, 1993.

[48] P. I. Pohl, J.-L. Faulon, and D. M. Smith, "Molecular dynamics computer simulations of silica aerogels," Journal of Non-Crystalline Solids, vol. 186, pp. 249-355, 1995.

[49] B. P. Feuston and S. H. Garofalini, "Oligomerization in silica sols," Journal of Physical Chemistry, vol. 94, no. 13, pp. 5351-5356, 1990.

[50] T. Campbell, R. K. Kalia, A. Nakano, F. Shimojo, K. Tsuruta, and P. Vashishta, "Structural correlations and mechanical behavior in nanophase silica glasses," Physical Review Letters, vol. 82, no. 20, pp. 4018-4021, 1999.

[51] S. Bhattacharya and J. Kieffer, "Fractal dimensions of silica gels generated using molecular dynamics simulations," The Journal of Chemical Physics, vol. 122, p. 094715, 2005.

[52] M. Moner-Girona, A. Roig, E. Molins, E. Martínez, and J. Esteve, "Micromechanical properties of silica aerogels," Applied Physics Letters, vol. 75, no. 5, pp. 653-655, 1999. 
[53] L. D. Gelb, "Simulating silica aerogels with a coarse-grained flexible model and Langevin dynamics," Journal of Physical Chemistry, vol. 111, no. 43, pp. 15792-15802, 2007.

[54] H. S. Ma, J. H. Prévost, R. Jullien, and G. W. Scherer, "Computer simulation of mechanicalstructure property relationship of aerogels," Journal of Non-Crystalline Solids, vol. 285, pp. 216-221, 2001.

[55] K. Muralidarhan, J. H. Simmons, P. A. Deymier, and K. Runge, "Molecular dynamics studies of brittle fracture in vitreous silica: Review and recent progress," Journal of Non-Crystalline Solids, vol. 351, pp. 1532-1542, 2005.

[56] T. F. Soules, "A molecular dynamics calculation of the structure of sodium silicate glasses," Journal of Chemical Physics, vol. 71, no. 11, pp. 4570-4578, 1979.

[57] B. W. H. van Beest, G. J. Kramer, and R. A. van Santen, "Force fields for silicas and aluminophosphates based on ab initio calculations," Physical Review Letters, vol. 64, no. 16, pp. $1955-1958,1990$.

[58] B. P. Feuston and S. H. Garofalini, "Empirical three-body potential for vitreous silica," Journal of Chemical Physics, vol. 89, no. 9, pp. 5818-5824, 1989.

[59] J. S. Rivas Murillo, E. J. Barbero, M. E. Bachlechner, and D. Cairns, "Toward toughening and understanding of silica aerogels MD simulations," in Proceedings of IMECE2008, ASME International Mechanical Engineering Congress and Exposition, (Oct. $31^{\text {st }}-$ Nov. $6^{\text {th }} 2008$, Boston, MA, USA), 2008.

[60] R. W. G. Wyckoff, Crystal structures, vol. 1. Intersciense Publishers, $2^{\text {nd }}$ ed., 1963.

[61] B. M. Lee, H. K. Baik, B. S. Seong, S. Munetoh, and T. Motooka, "Generation of glass $\mathrm{SiO}_{2}$ structures by various cooling rates: A molecular-dynamics study," Computational Materials Science, vol. 37, pp. 203-208, 2006.

[62] R. Sayle and E. J. Milner White, "RasMol: Biomolecular graphics for all," Trends in Biochemical Sciences (TIBS), vol. 20, no. 9, p. 374, 1995. 
[63] H. J. C. Berendsen, J. P. M. Postma, W. F. van Gunsteren, A. DiNola, and J. R. Haak, "Molecular dynamics with coupling to an external bath," Journal of Chemical Physics, vol. 81, no. 8, pp. 3684-3690, 1984 .

[64] J. S. Rivas Murillo, M. E. Bachlechner, F. A. Campo, and E. J. Barbero, "Structure and mechanical properties of silica aerogels and xerogels modeled by molecular dynamics simulations," Journal of Non-Crystalline Solids, vol. 356, no. 25-27, pp. 1325-1331, 2010.

[65] C. L. Rountree, Massively parallel molecular dynamics simulations of crack-front dynamics and morphology in amorphous nanostructured silica. $\mathrm{PhD}$ thesis, Agricultural and Mechanical College, Louisiana State University, Dec. 2003.

[66] D. W. Brenner, O. A. Shenderova, J. A. Harrison, S. J. Stuart, B. Ni, and S. B. Sinnott, "A second-generation reactive empirical bond order (REBO) potential energy expression for hydrocarbons," Journal of Physics: Condensed Matter, vol. 14, pp. 783-802, 2002.

[67] S. J. Stuart, A. B. Tutein, and J. A. Harrison, "A reactive potential for hydrocarbons with intermolecular interactions," Journal of Chemical Physics, vol. 112, no. 14, pp. 6472-6486, 2000.

[68] N. Boris, K. H. Lee, and S. B. Sinnott, "A reactive empirical bond order (REBO) potential for hydrocarbon-oxygen interactions," Journal of Physics: Condensed Matter, vol. 16, pp. 7261$7275,2004$.

[69] A. J. Dyson and P. V. Smith, "Extension of the Brenner empirical interatomic potential to C-Si-H systems," Surface Science, vol. 355, pp. 140-150, 1996.

[70] L. Qi and S. B. Sinnott, "Polymerization via cluster-solid surface impacts: Molecular dynamics simulations," Journal of Physical Chemistry B, vol. 101, no. 35, pp. 6883-6890, 1997.

[71] A. B. Tutein, S. J. Stuart, and J. A. Harrison, "Indentation analysis of linear-chain hydrocarbon monolayers anchored to diamond," Journal of Physical Chemistry B, vol. 103, no. 51, pp. 11357-11365, 1999. 
[72] O. A. Shenderova and D. W. Brenner, "Atomistic simulations of structures and mechanical properties of $<011>$ tilt grain boundaries and their triple junctions in diamond," Physical Review B: Condensed Matter, vol. 60, no. 10, pp. 7053-7061, 1999.

[73] O. A. Shenderova, D. W. Brenner, A. Omeltchenko, X. Su, and L. H. Yang, "Atomistic modeling of the fracture of polycrystalline diamond," Physical Review B: Condensed Matter, vol. 61, no. 6 , pp. $3877-3888,2000$.

[74] S. B. Sinnott, R. J. Colton, C. T. White, O. A. Shenderova, D. W. Brenner, and J. A. Harrison, "Atomistic simulations of the nanometer-scale indentation of amorphous-carbon thin films," Journal of Vacuum Science \& Technology A: Vacuum, Surfaces, and Films, vol. 15, no. 3, pp. 936-940, 1997.

[75] D. W. Brenner, "Empirical potential for hydrocarbons for use in simulating the chemical vapor deposition of diamond films," Physical Review B, vol. 42, no. 15, pp. 9458-9471, 1990.

[76] A. J. Dyson and P. V. Smith, "Empirical potential study of the chemisorption of $\mathrm{C}_{2} \mathrm{H}_{2}$ and $\mathrm{CH}_{3}$ on the $\beta$-SiC(001) surface," Surface Science, vol. 396, pp. 24-39, 1998.

[77] A. J. Dyson and P. V. Smith, "A molecular dynamics study of the chemisorption of $\mathrm{C}_{2} \mathrm{H}_{2}$ and $\mathrm{CH}_{3}$ on the $\mathrm{Si}(001)-(2 \times 1)$ surface," Surface Science, vol. 375, pp. 45-54, 1997. 
Appendix A

Additional Figures: Samples 

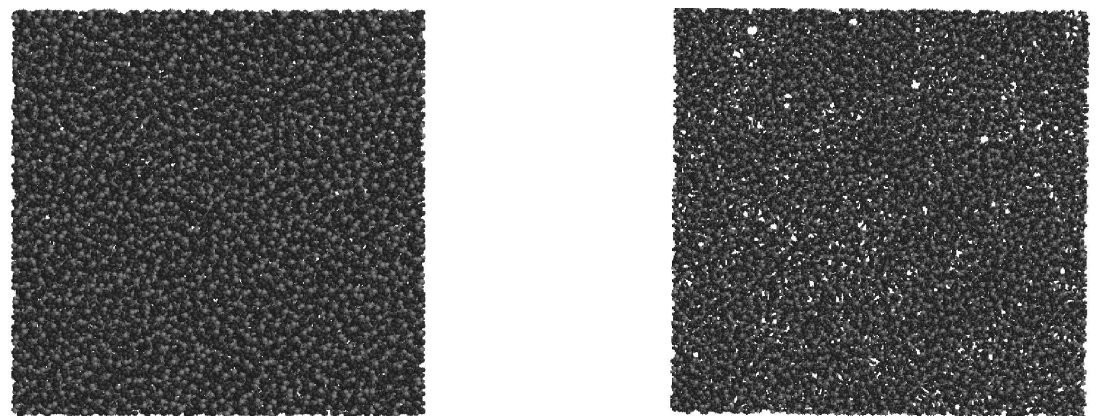

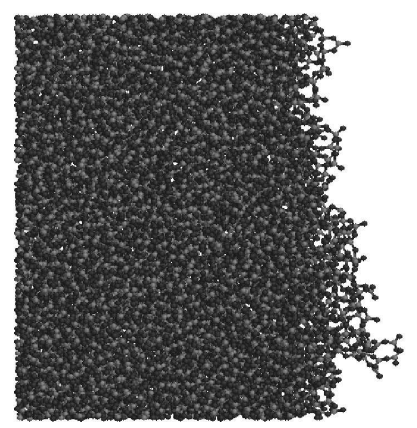

(a)
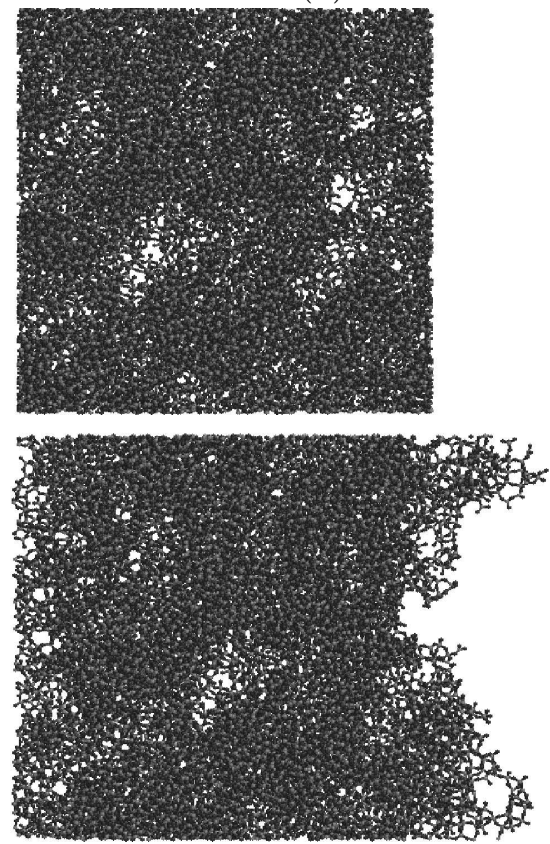

(c)
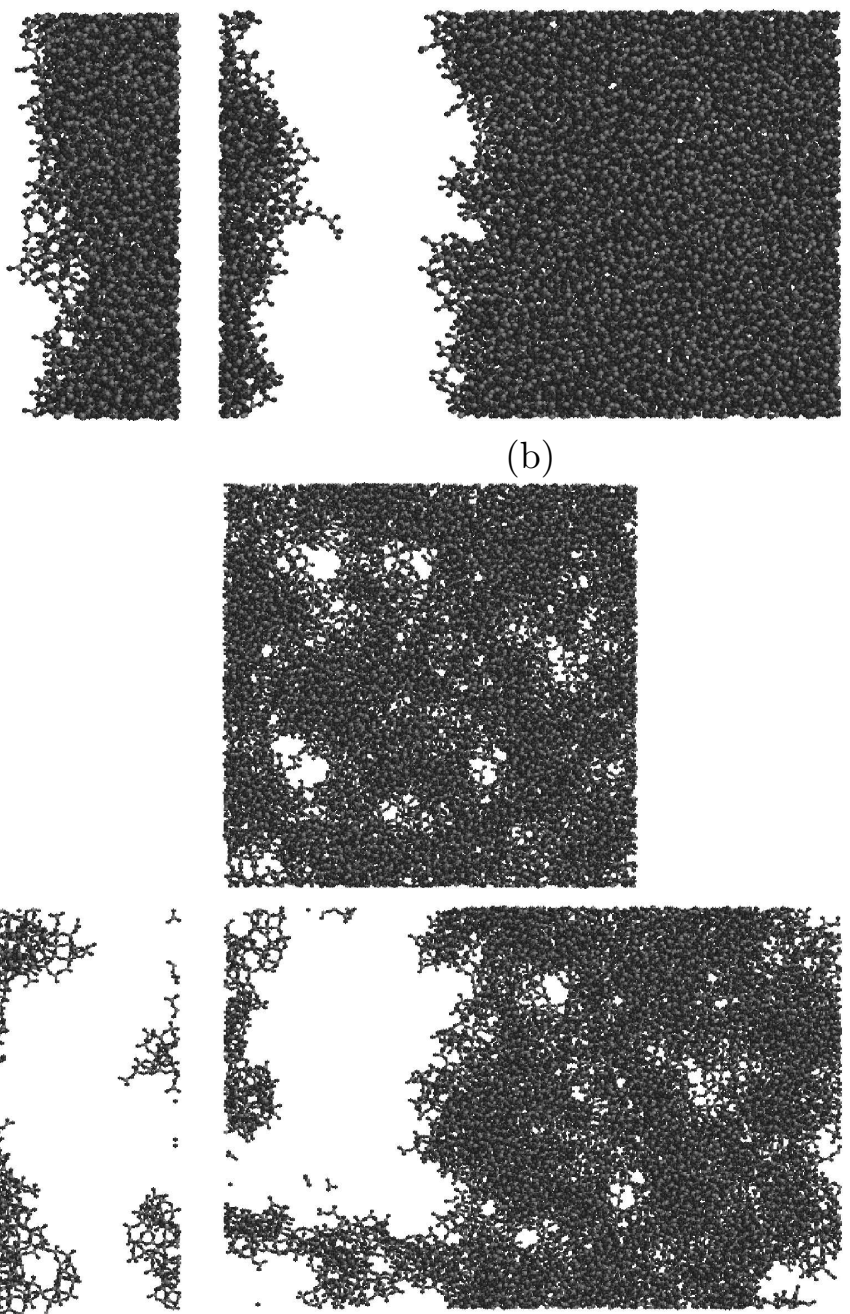

(d)

Figure A.1: Silica aerogel and xerogel samples before (above) and after (below) tension test 62]. (a) $\rho=$ $2.20 \frac{g}{\mathrm{~cm}^{3}}$, (b) $\rho=1.49 \frac{\mathrm{g}}{\mathrm{cm}^{3}}$, (c) $\rho=1.05 \frac{\mathrm{g}}{\mathrm{cm}^{3}}$, (d) $\rho=0.77 \frac{\mathrm{g}}{\mathrm{cm}^{3}}$. 

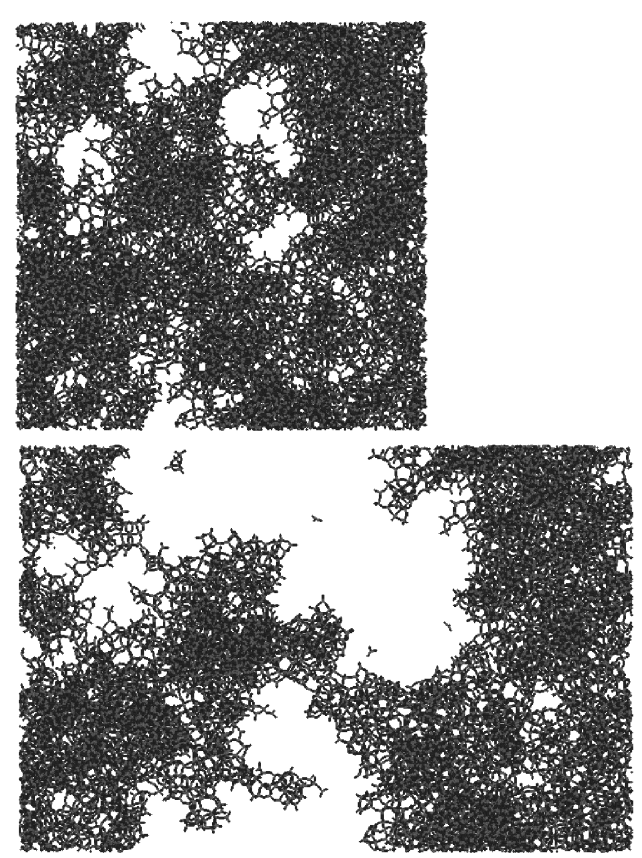

(a)
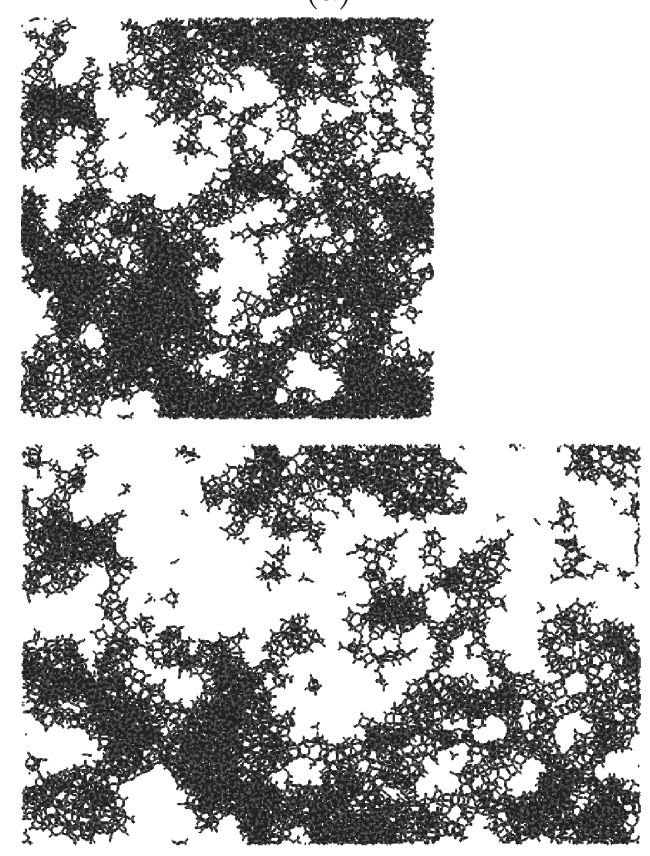

(c)
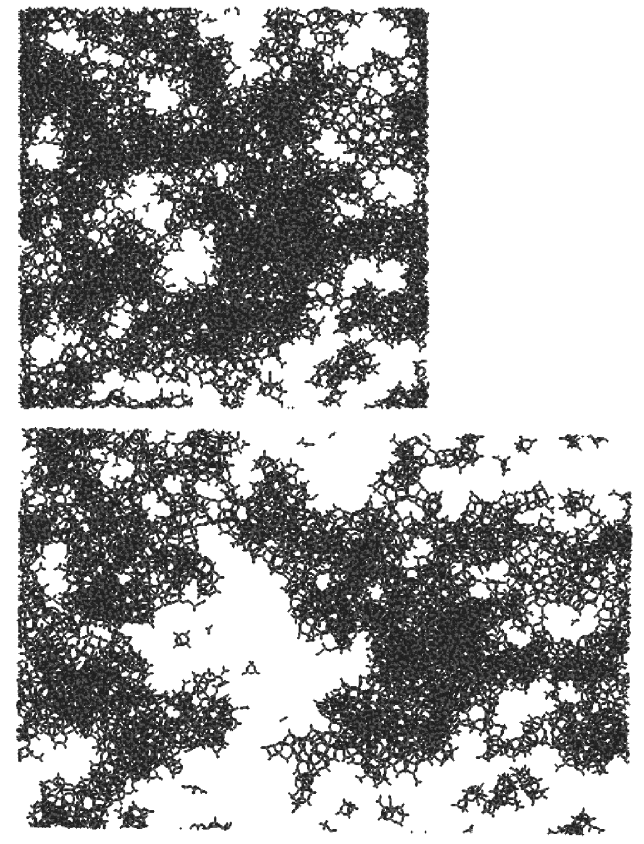

(b)
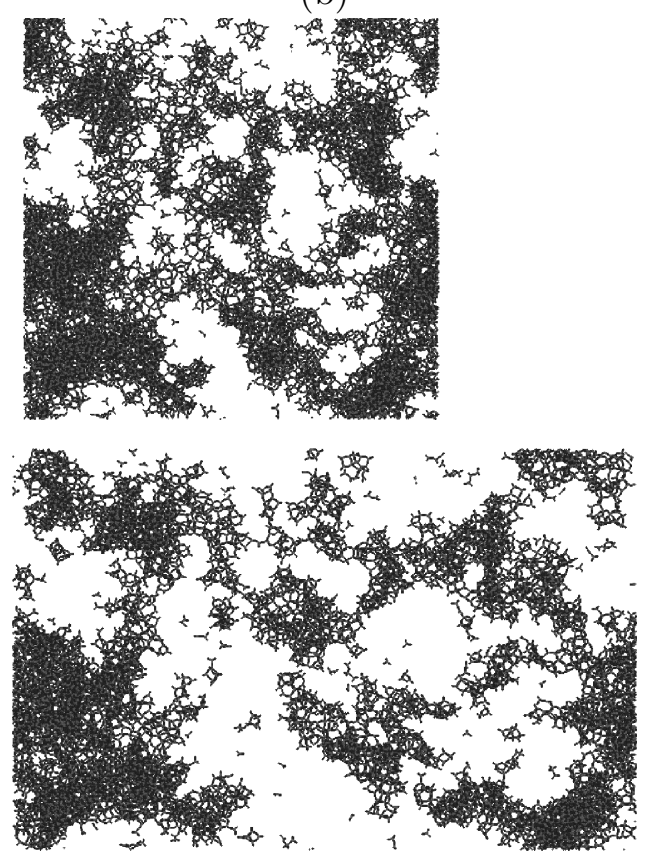

(d)

Figure A.2: Silica aerogel and xerogel samples before (above) and after (below) tension test 62]. (a) $\rho=$ $0.58 \frac{g}{\mathrm{~cm}^{3}}$, (b) $\rho=0.35 \frac{g}{\mathrm{~cm}^{3}}$, (c) $\rho=0.28 \frac{g}{\mathrm{~cm}^{3}}$, (d) $\rho=0.23 \frac{g}{\mathrm{~cm}^{3}}$. 

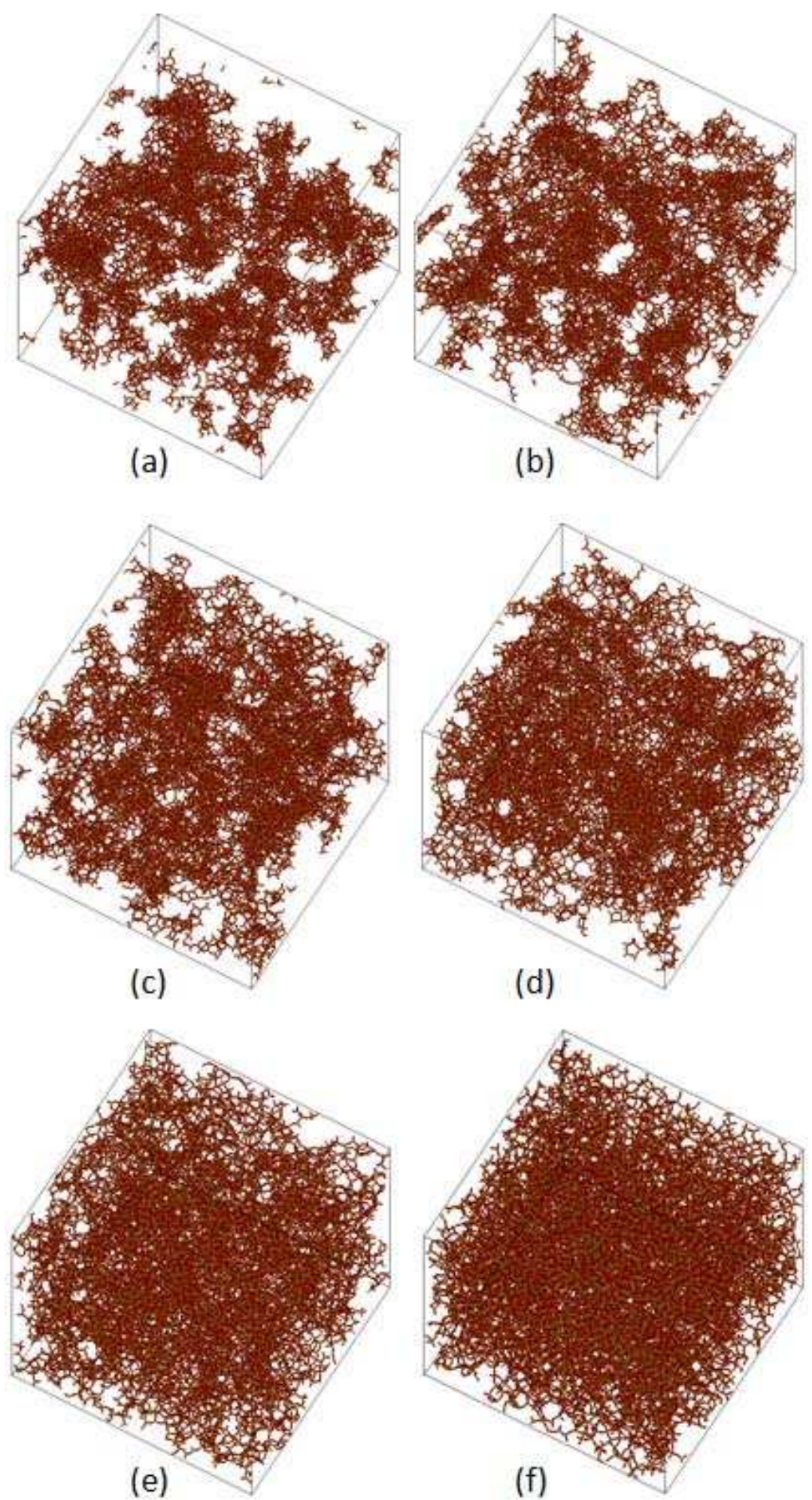

Figure A.3: Silica aerogel and xerogel samples before tension test 62. (a) $\rho=0.35 \frac{\mathrm{g}}{\mathrm{cm}^{3}}$, (b) $\rho=0.45 \frac{\mathrm{g}}{\mathrm{cm}^{3}}$, (c) $\rho=0.58 \frac{g}{\mathrm{~cm}^{3}}$, (d) $\rho=0.77 \frac{\mathrm{g}}{\mathrm{cm}^{3}}$, (e) $\rho=1.05 \frac{\mathrm{g}}{\mathrm{cm}^{3}}$, (f) $\rho=1.49 \frac{\mathrm{g}}{\mathrm{cm}^{3}}$ 

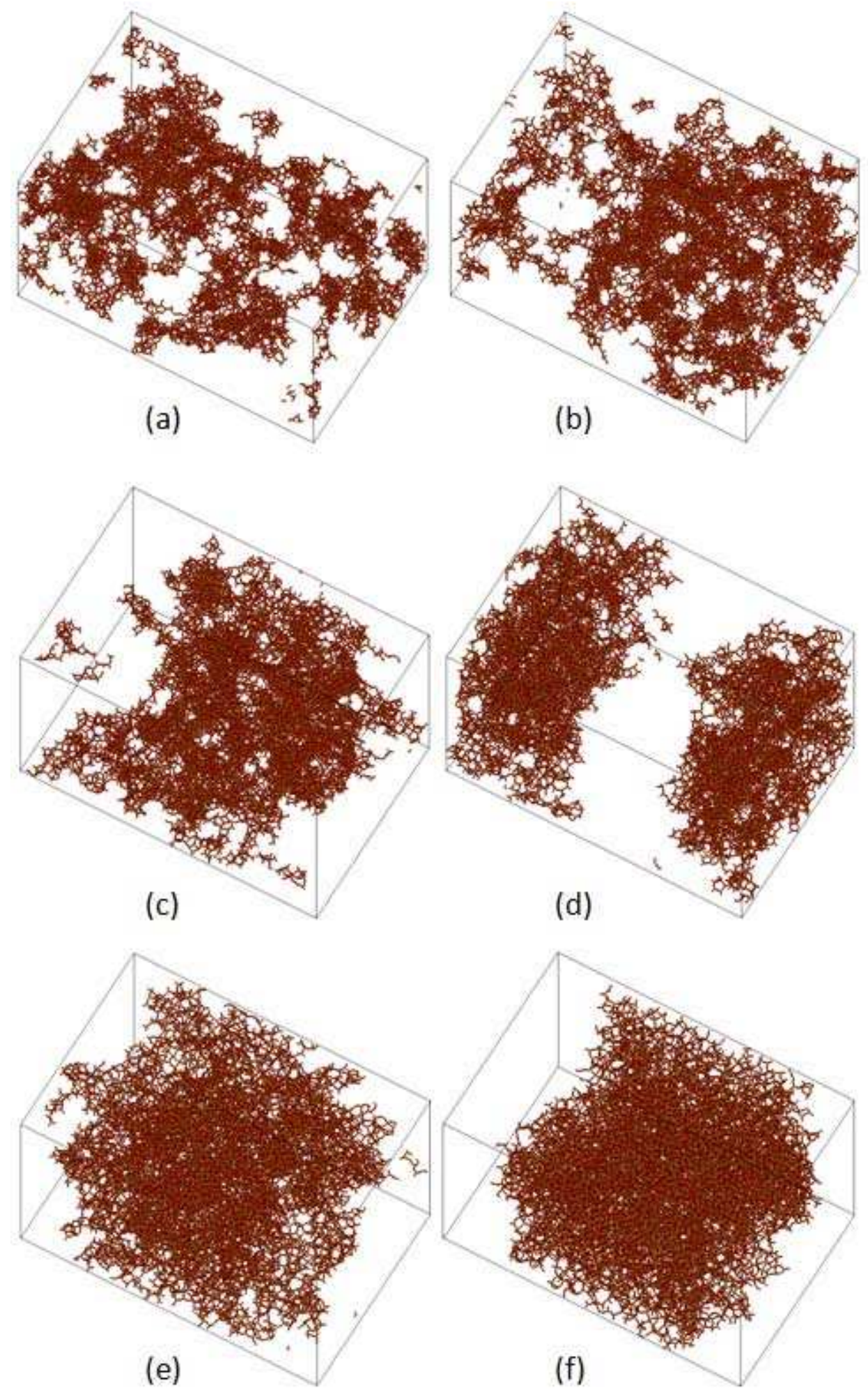

Figure A.4: Silica aerogel and xerogel samples after tension test [62. (a) $\rho=0.35 \frac{g}{\mathrm{~cm}^{3}}$, (b) $\rho=0.45 \frac{g}{\mathrm{~cm}^{3}}$, (c) $\rho=0.58 \frac{g}{\mathrm{~cm}^{3}}$, (d) $\rho=0.77 \frac{g}{\mathrm{~cm}^{3}}$, (e) $\rho=1.05 \frac{g}{\mathrm{~cm}^{3}}$, (f) $\rho=1.49 \frac{g}{\mathrm{~cm}^{3}}$ 

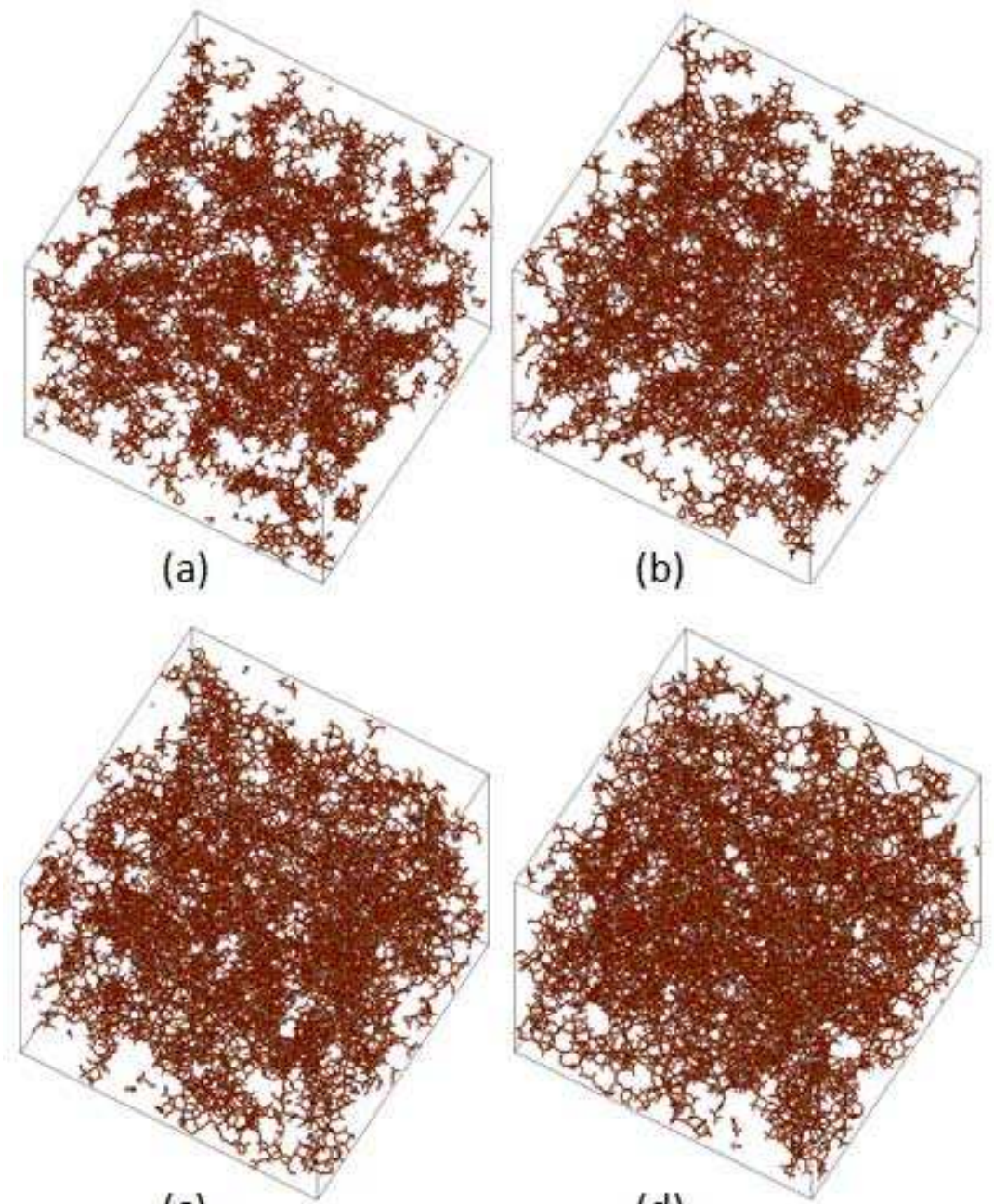

(c)

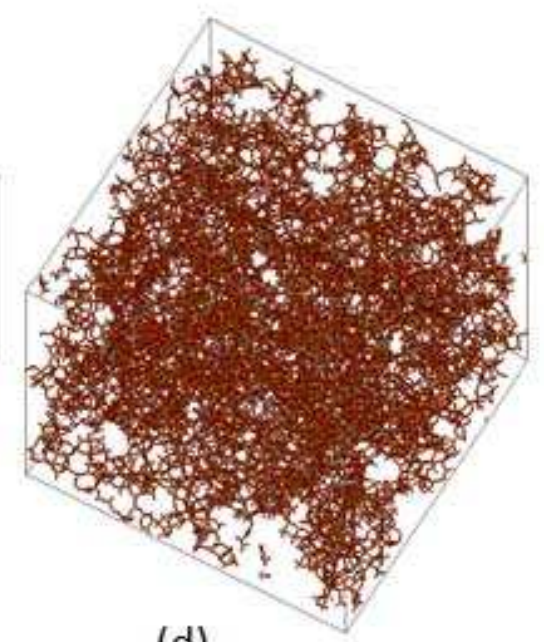

(d)
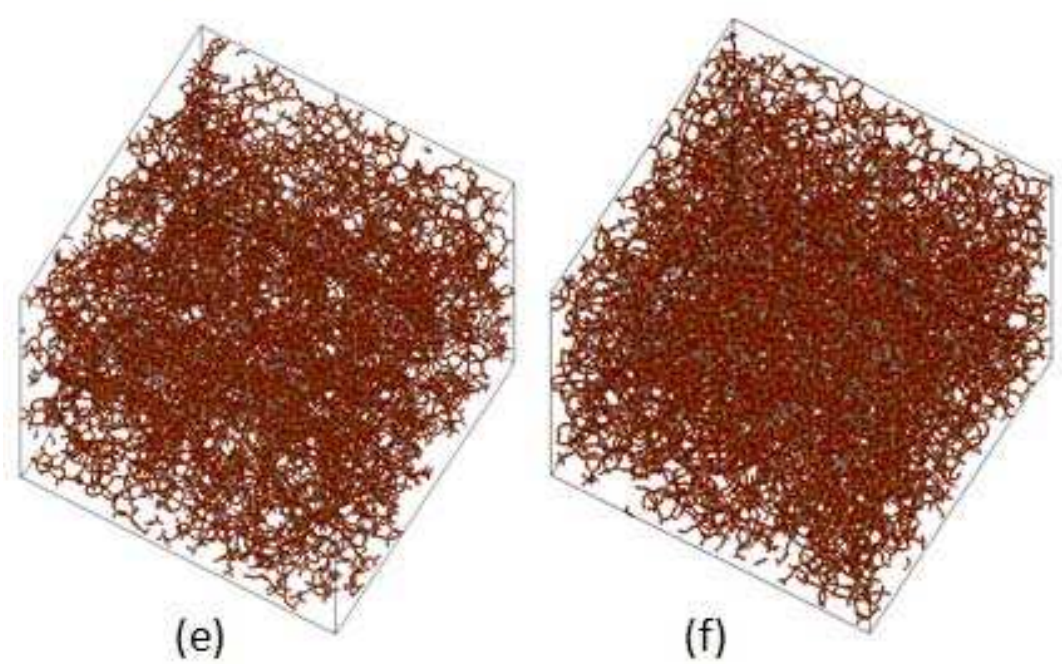

Figure A.5: Hybrid aerogel and xerogel samples before tension test 62, $0.1 \mathrm{~mol} \% \mathrm{CH}_{3}$ bonded to $\mathrm{Si}$. (a) $\rho=0.35 \frac{g}{\mathrm{~cm}^{3}}$, (b) $\rho=0.45 \frac{g}{\mathrm{~cm}^{3}}$, (c) $\rho=0.58 \frac{g}{\mathrm{~cm}^{3}}$, (d) $\rho=0.77 \frac{g}{\mathrm{~cm}^{3}}$, (e) $\rho=1.05 \frac{g}{\mathrm{~cm}^{3}}$, (f) $\rho=1.49 \frac{g}{\mathrm{~cm}^{3}}$ 

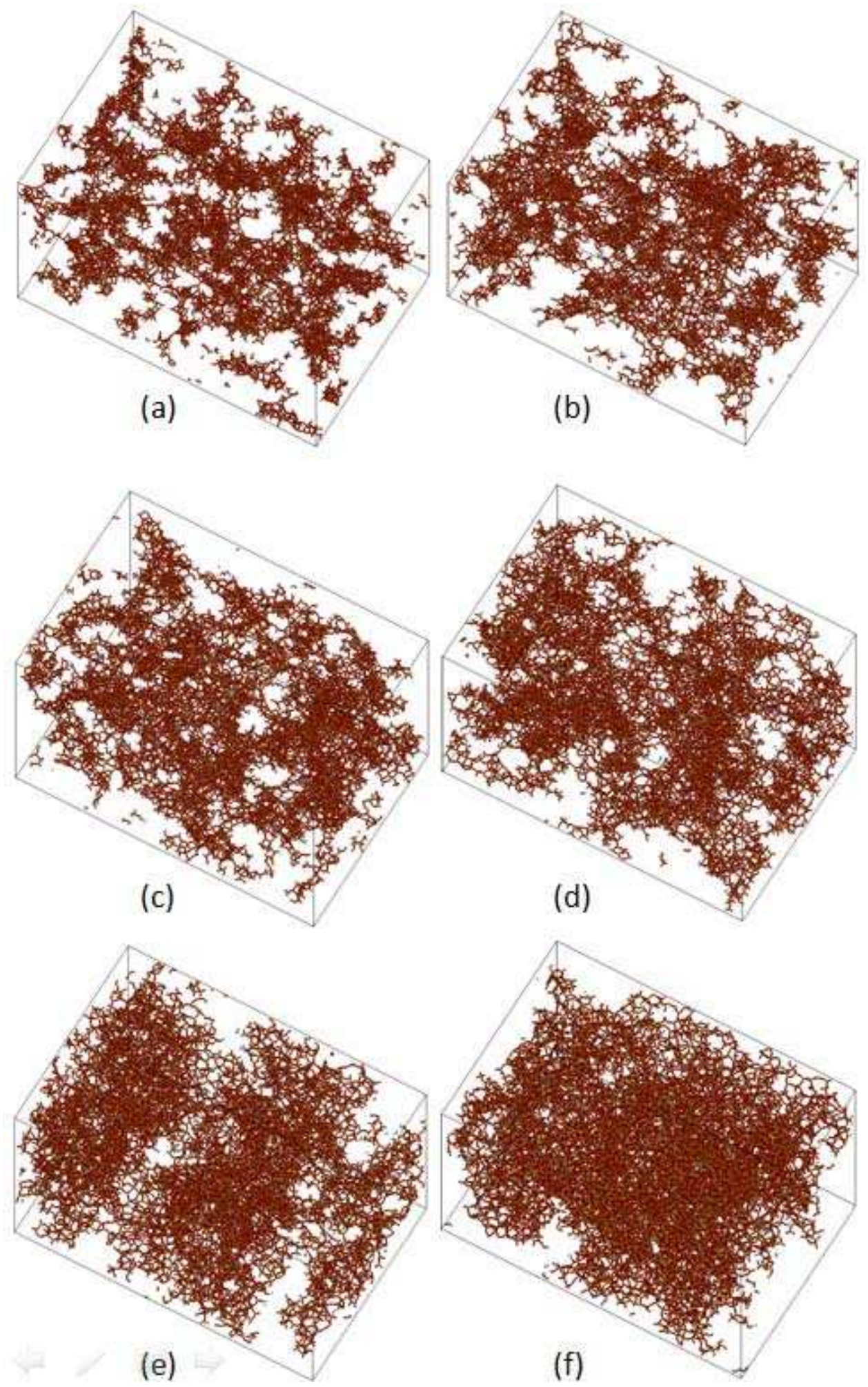

Figure A.6: Hybrid aerogel and xerogel samples after tension test 62, $0.1 \mathrm{~mol} \% \mathrm{CH}_{3}$ bonded to Si. (a) $\rho=0.35 \frac{g}{\mathrm{~cm}^{3}}$, (b) $\rho=0.45 \frac{g}{\mathrm{~cm}^{3}}$, (c) $\rho=0.58 \frac{g}{\mathrm{~cm}^{3}}$, (d) $\rho=0.77 \frac{g}{\mathrm{~cm}^{3}}$, (e) $\rho=1.05 \frac{g}{\mathrm{~cm}^{3}}$, (f) $\rho=1.49 \frac{\mathrm{g}}{\mathrm{cm}^{3}}$ 


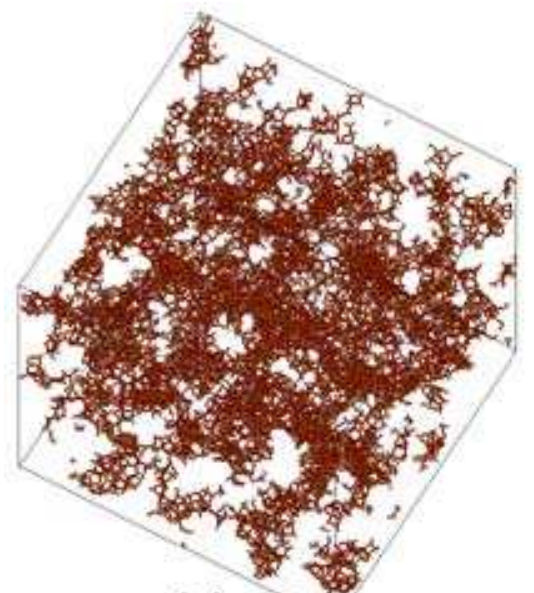

(a)
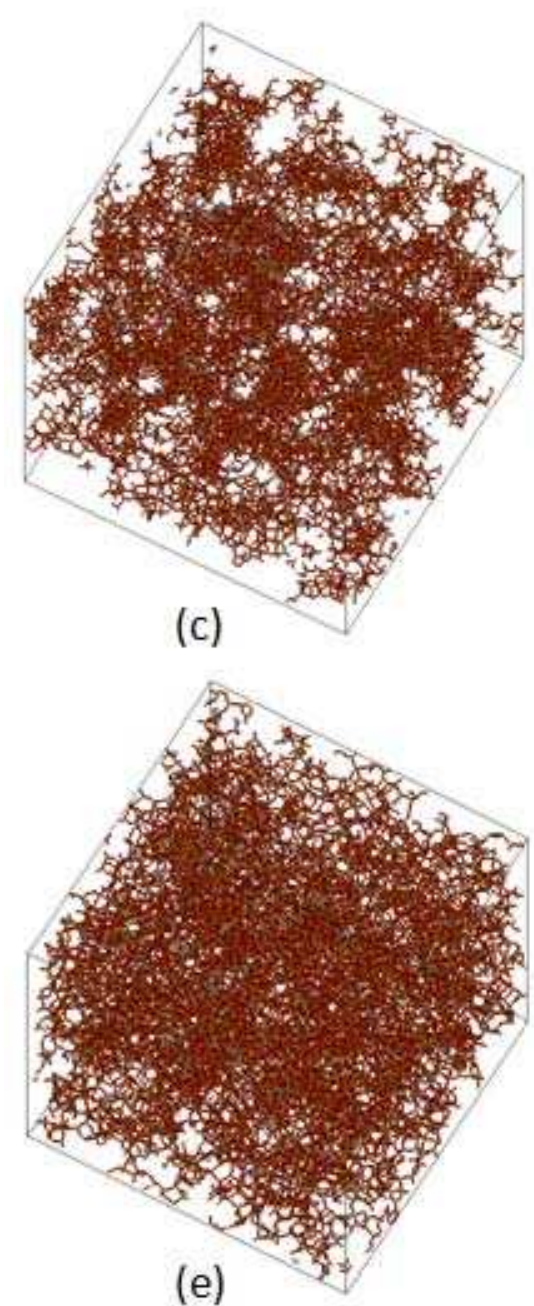
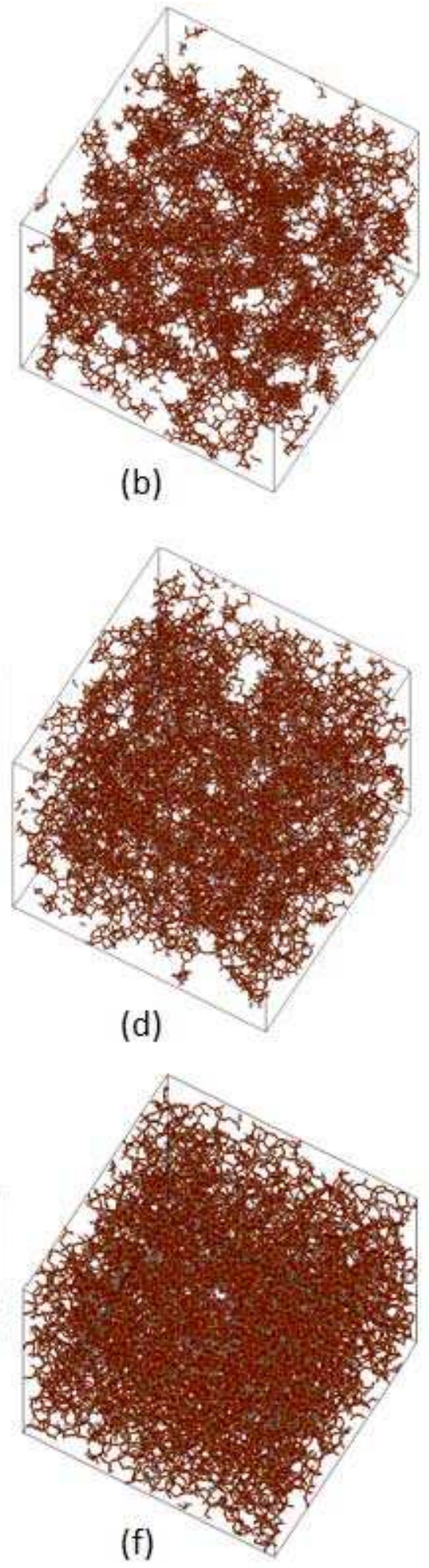

Figure A.7: Hybrid aerogel and xerogel samples before tension test [62, $0.1 \mathrm{~mol} \% \mathrm{CH}_{3}$ bonded to O. (a) $\rho=0.35 \frac{g}{\mathrm{~cm}^{3}}$, (b) $\rho=0.45 \frac{g}{\mathrm{~cm}^{3}}$, (c) $\rho=0.58 \frac{\mathrm{g}}{\mathrm{cm}^{3}}$, (d) $\rho=0.77 \frac{\mathrm{g}}{\mathrm{cm}^{3}}$, (e) $\rho=1.05 \frac{\mathrm{g}}{\mathrm{cm}^{3}}$, (f) $\rho=1.49 \frac{\mathrm{g}}{\mathrm{cm}^{3}}$ 

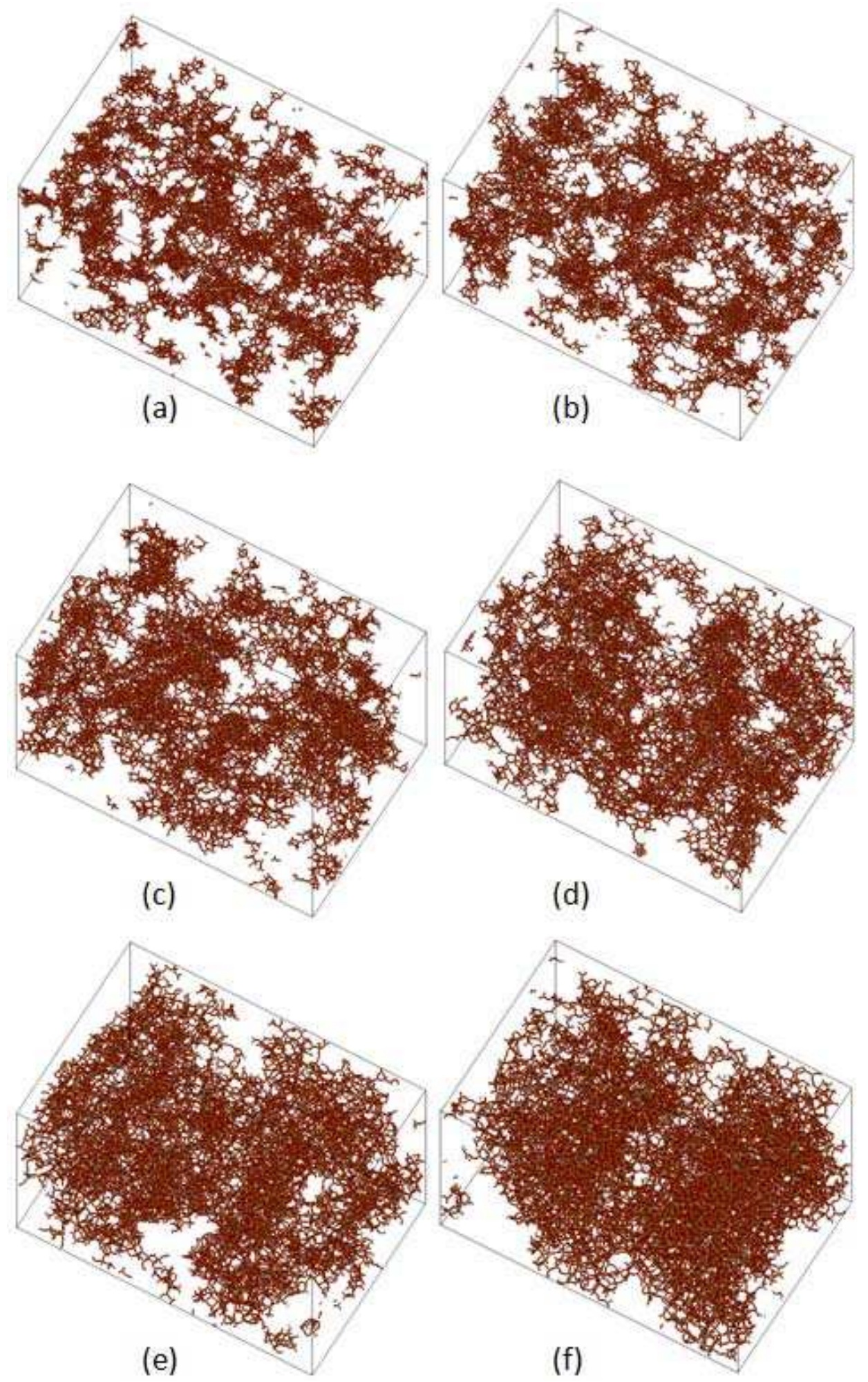

Figure A.8: Hybrid aerogel and xerogel samples after tension test 62, $0.1 \mathrm{~mol} \% \mathrm{CH}_{3}$ bonded to O. (a) $\rho=$ $0.35 \frac{g}{c m^{3}}$, (b) $\rho=0.45 \frac{g}{c m^{3}}$, (c) $\rho=0.58 \frac{g}{c^{3}}$, (d) $\rho=0.77 \frac{g}{c^{3}}$, (e) $\rho=1.05 \frac{g}{c^{3}}$, (f) $\rho=1.49 \frac{g}{c^{3}}$ 

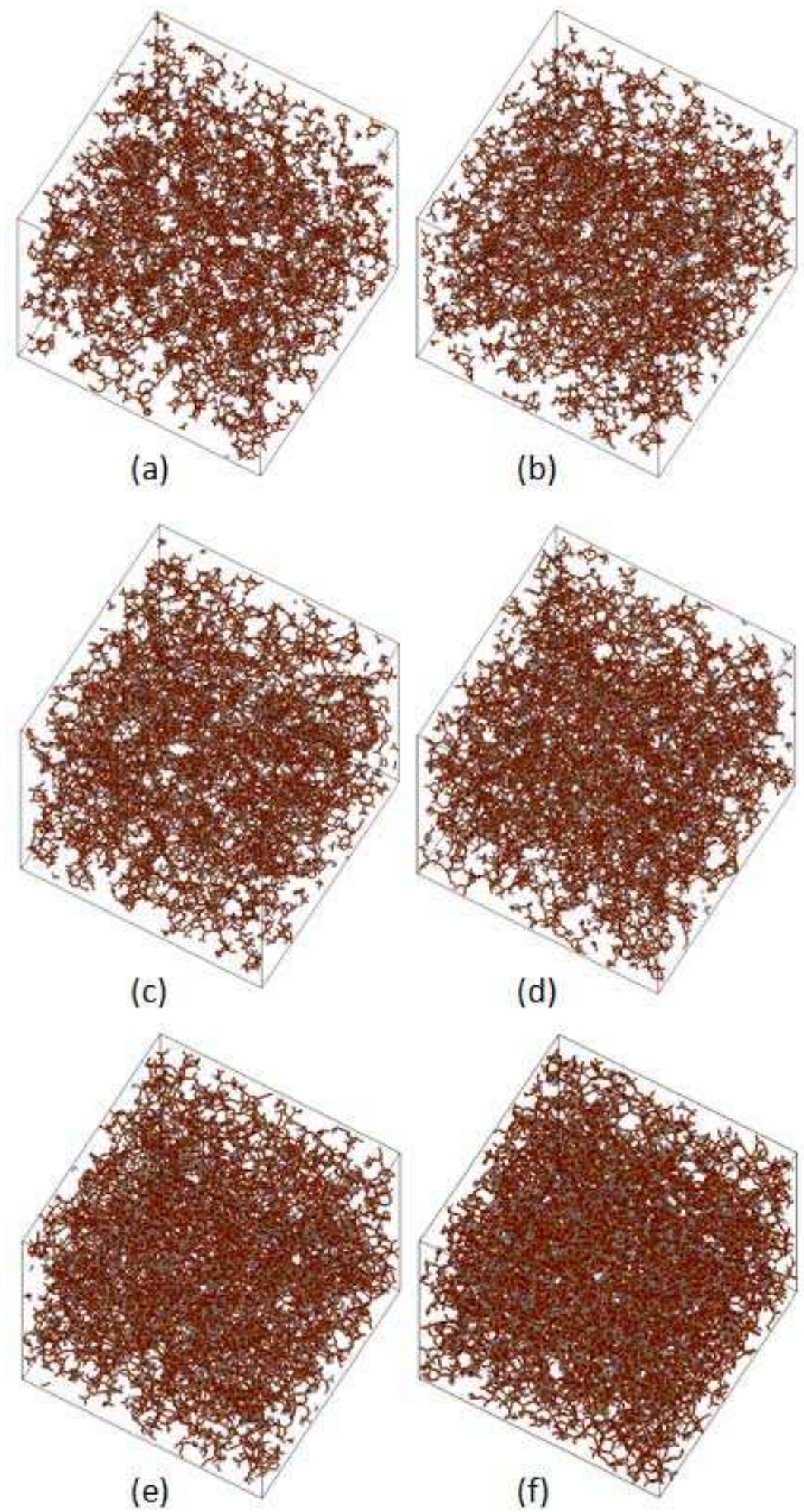

Figure A.9: Hybrid aerogel and xerogel samples before tension test 62, $0.4 \mathrm{~mol} \% \mathrm{CH}_{3}$ bonded to Si. (a) $\rho=0.35 \frac{g}{\mathrm{~cm}^{3}}$, (b) $\rho=0.45 \frac{g}{\mathrm{~cm}^{3}}$, (c) $\rho=0.58 \frac{\mathrm{g}}{\mathrm{cm}^{3}}$, (d) $\rho=0.77 \frac{\mathrm{g}}{\mathrm{cm}^{3}}$, (e) $\rho=1.05 \frac{\mathrm{g}}{\mathrm{cm}^{3}}$, (f) $\rho=1.49 \frac{\mathrm{g}}{\mathrm{cm}^{3}}$ 

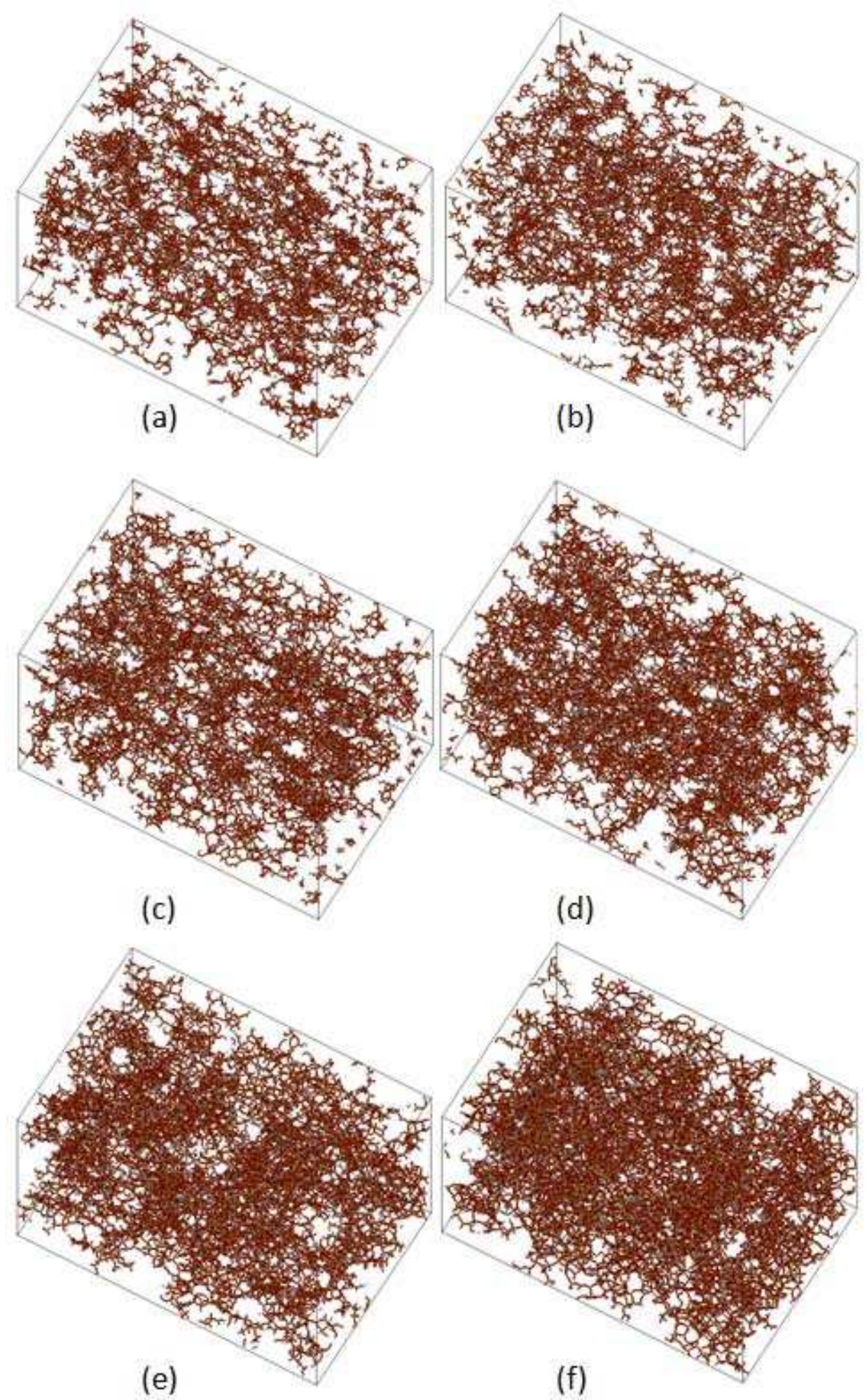

Figure A.10: Hybrid aerogel and xerogel samples after tension test 62, $0.4 \mathrm{~mol} \% \mathrm{CH}_{3}$ bonded to $\mathrm{Si}$. (a) $\rho=0.35 \frac{g}{\mathrm{~cm}^{3}}$, (b) $\rho=0.45 \frac{\mathrm{g}}{\mathrm{cm}^{3}}$, (c) $\rho=0.58 \frac{\mathrm{g}}{\mathrm{cm}^{3}}$, (d) $\rho=0.77 \frac{\mathrm{g}}{\mathrm{cm}^{3}}$, (e) $\rho=1.05 \frac{\mathrm{g}}{\mathrm{cm}^{3}}$, (f) $\rho=1.49 \frac{\mathrm{g}}{\mathrm{cm}^{3}}$ 

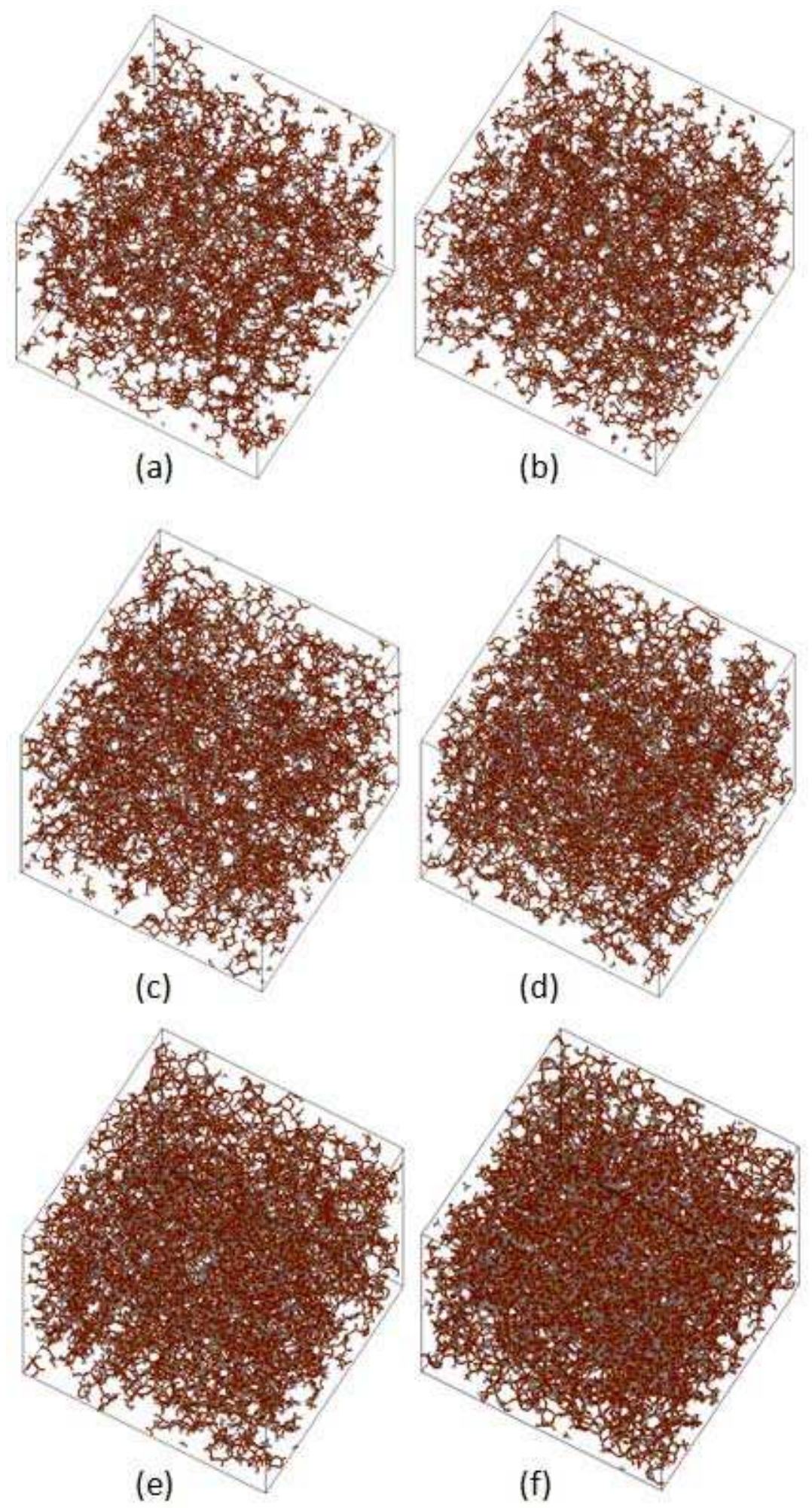

Figure A.11: Hybrid aerogel and xerogel samples before tension test 62, $0.4 \mathrm{~mol} \% \mathrm{CH}_{3}$ bonded to O. (a) $\rho=0.35 \frac{g}{\mathrm{~cm}^{3}}$, (b) $\rho=0.45 \frac{g}{\mathrm{~cm}^{3}}$, (c) $\rho=0.58 \frac{g}{\mathrm{~cm}^{3}}$, (d) $\rho=0.77 \frac{\mathrm{g}}{\mathrm{cm}^{3}}$, (e) $\rho=1.05 \frac{g}{\mathrm{~cm}^{3}}$, (f) $\rho=1.49 \frac{\mathrm{g}}{\mathrm{cm}^{3}}$ 

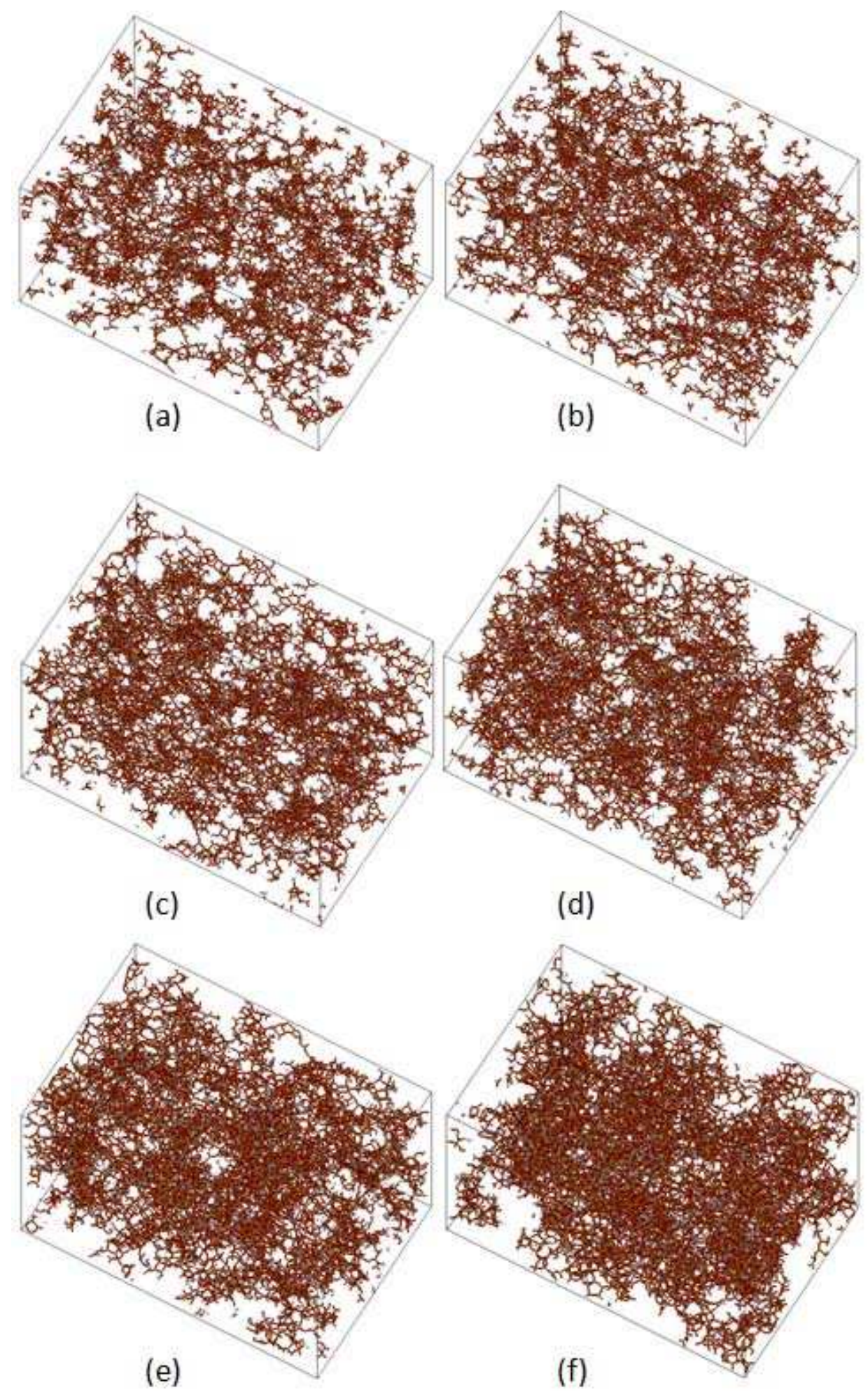

Figure A.12: Hybrid aerogel and xerogel samples after tension test 62, $0.4 \mathrm{~mol} \% \mathrm{CH}_{3}$ bonded to O. (a) $\rho=0.35 \frac{g}{\mathrm{~cm}^{3}}$, (b) $\rho=0.45 \frac{\mathrm{g}}{\mathrm{cm}^{3}}$, (c) $\rho=0.58 \frac{\mathrm{g}}{\mathrm{cm}^{3}}$, (d) $\rho=0.77 \frac{\mathrm{g}}{\mathrm{cm}^{3}}$, (e) $\rho=1.05 \frac{\mathrm{g}}{\mathrm{cm}^{3}}$, (f) $\rho=1.49 \frac{\mathrm{g}}{\mathrm{cm}^{3}}$ 
Appendix B

Additional Figures: Pair Distribution Function \& Fractal Dimension 


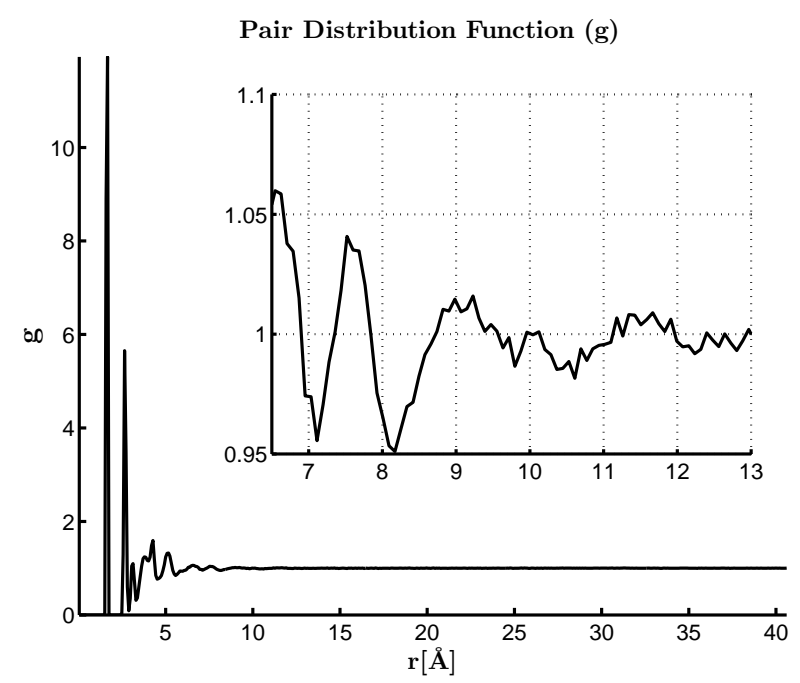

(a)

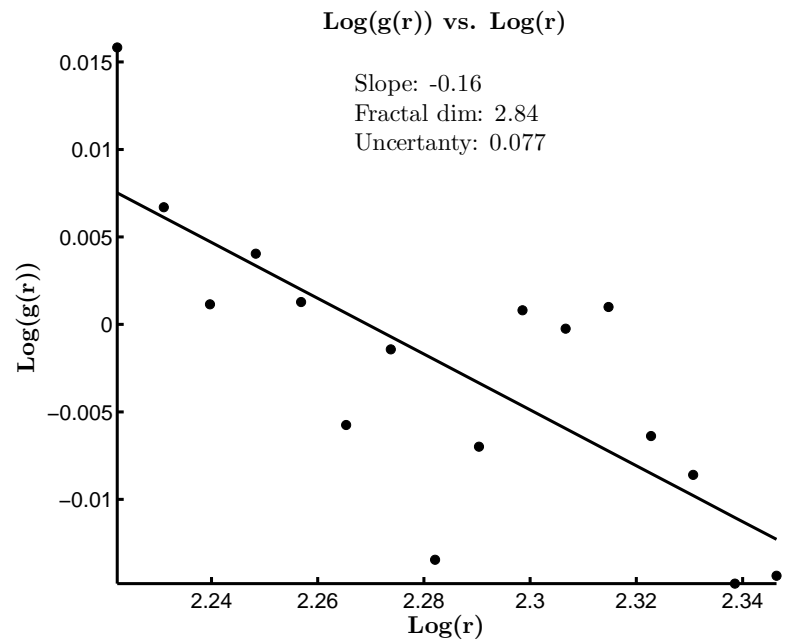

(b)

Figure B.1: Pair Distribution Function and fractal dimension for silica xerogel, $\rho=1.49 \frac{\mathrm{gr}}{\mathrm{cm}^{3}}$.

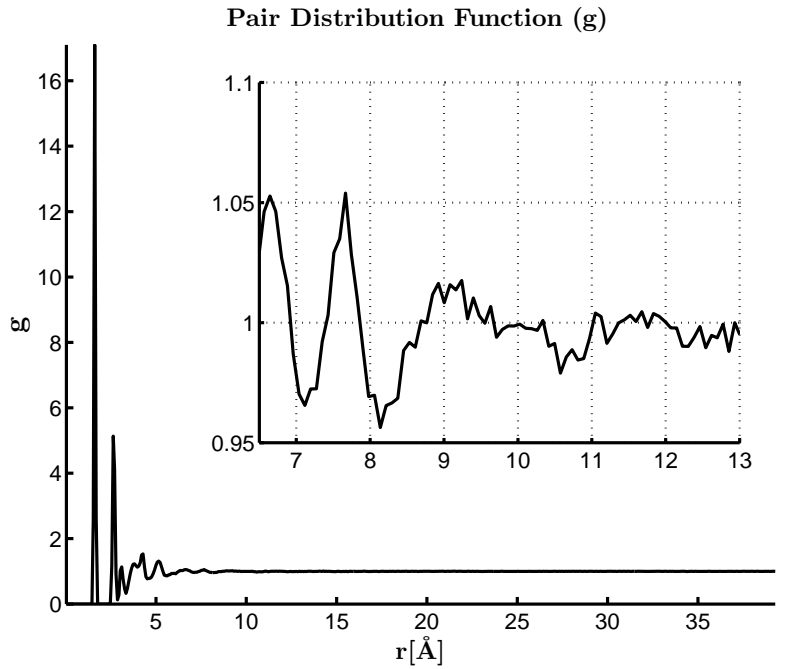

(a)

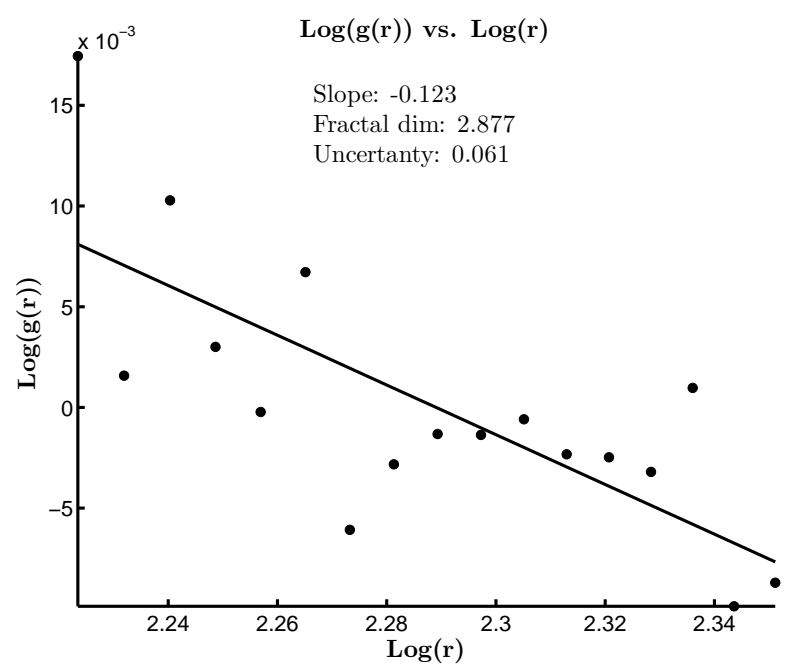

(b)

Figure B.2: Pair Distribution Function and fractal dimension for hybrid xerogel, $\rho=1.49 \frac{g r}{\mathrm{~cm}^{3}}, 0.1 \mathrm{~mol} \%$ $\mathrm{CH}_{3}$ bonded to $\mathrm{Si}$. 


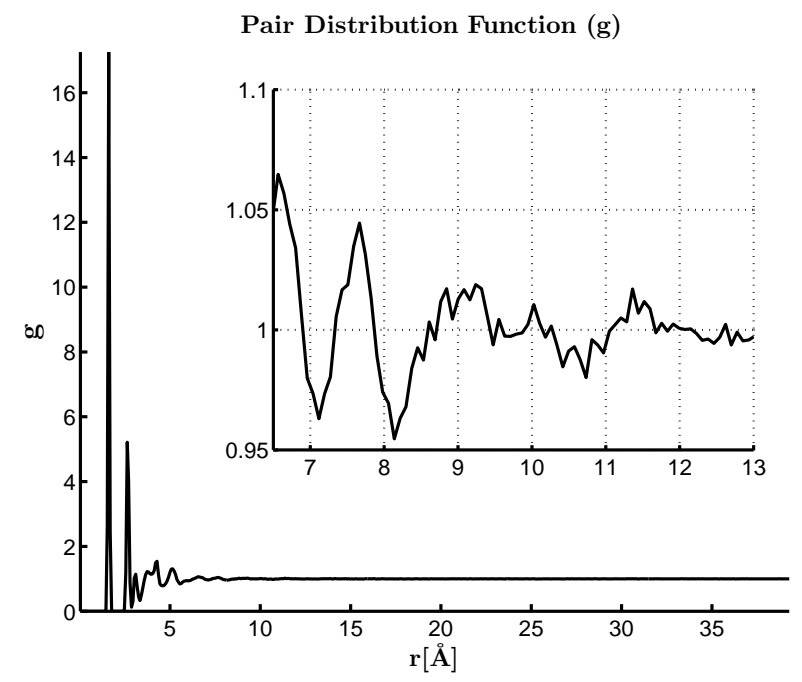

(a)

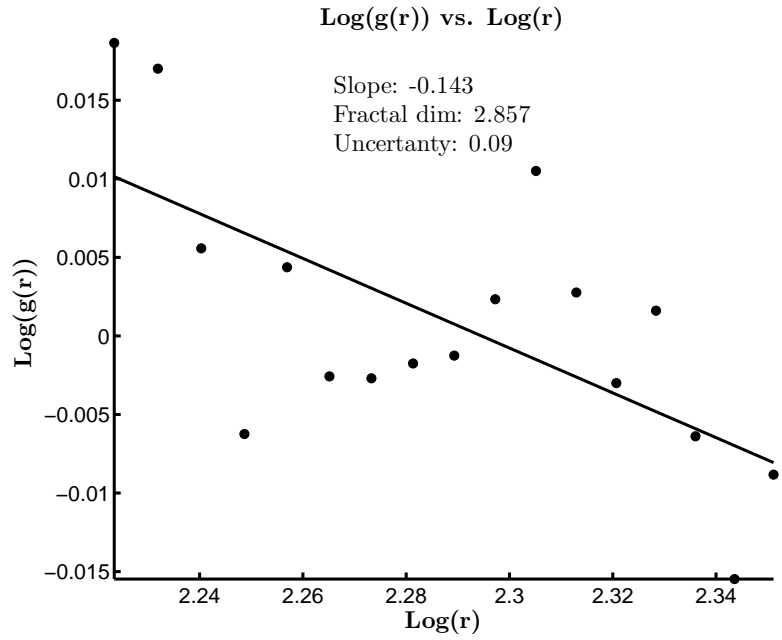

(b)

Figure B.3: Pair Distribution Function and fractal dimension for hybrid xerogel, $\rho=1.49 \frac{g r}{\mathrm{~cm}^{3}}, 0.1 \mathrm{~mol} \%$ $\mathrm{CH}_{3}$ bonded to $\mathrm{O}$.

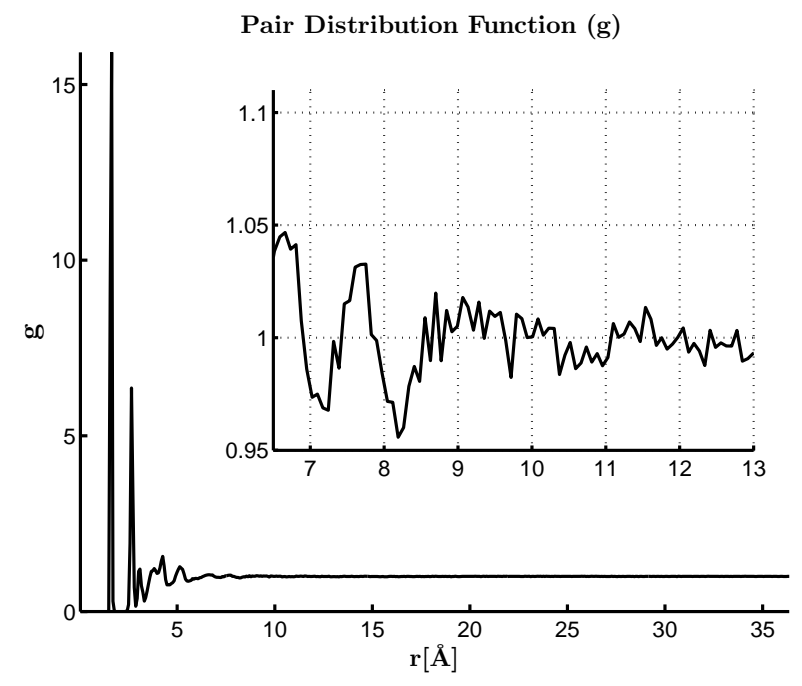

(a)

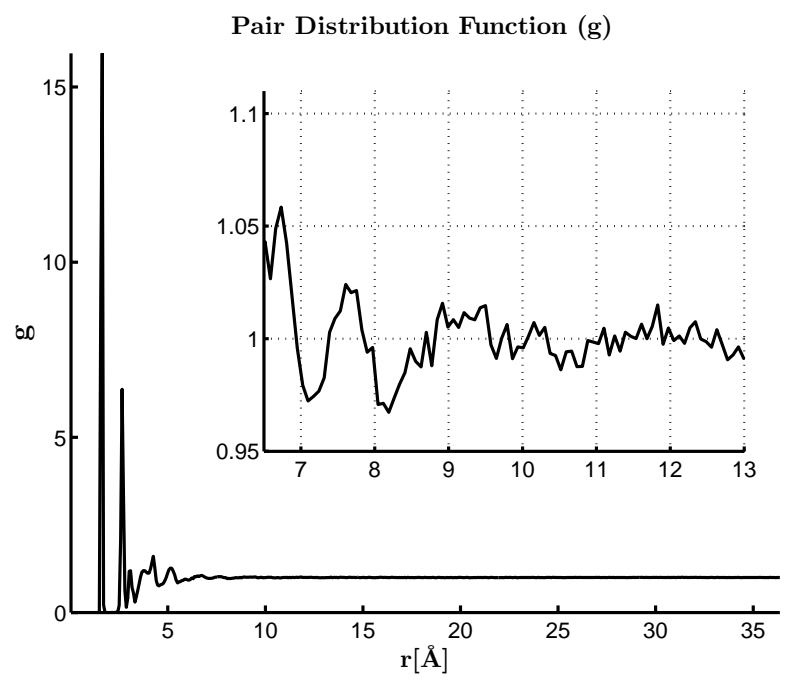

(b)

Figure B.4: Pair Distribution Function hybrid aerogel, $\rho=1.49 \frac{g}{\mathrm{~cm}^{3}}, 0.4 \mathrm{~mol} \% \mathrm{CH}_{3}$ content. (a) $\mathrm{CH}_{3}$ bonded to $\mathrm{Si}$, (b) $\mathrm{CH}_{3}$ bonded to $\mathrm{O}$. 


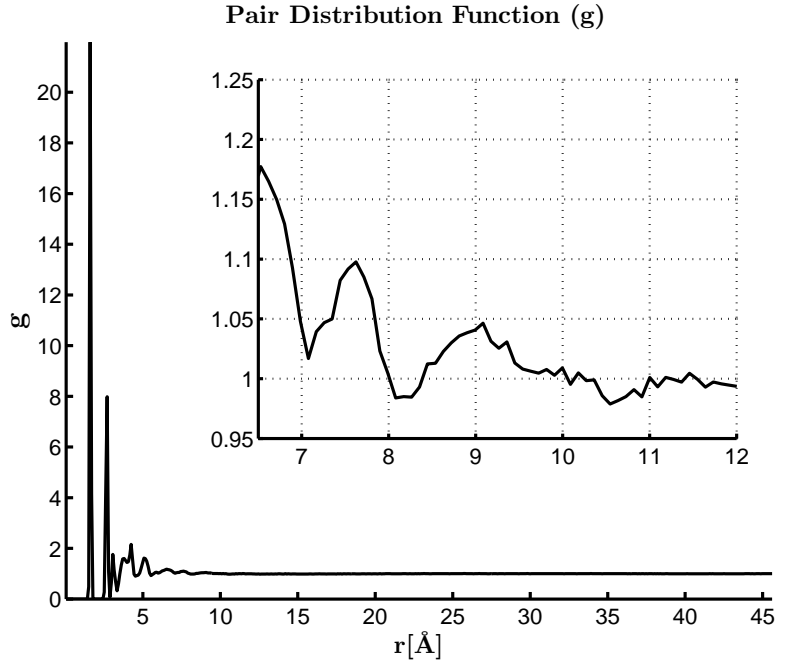

(a)

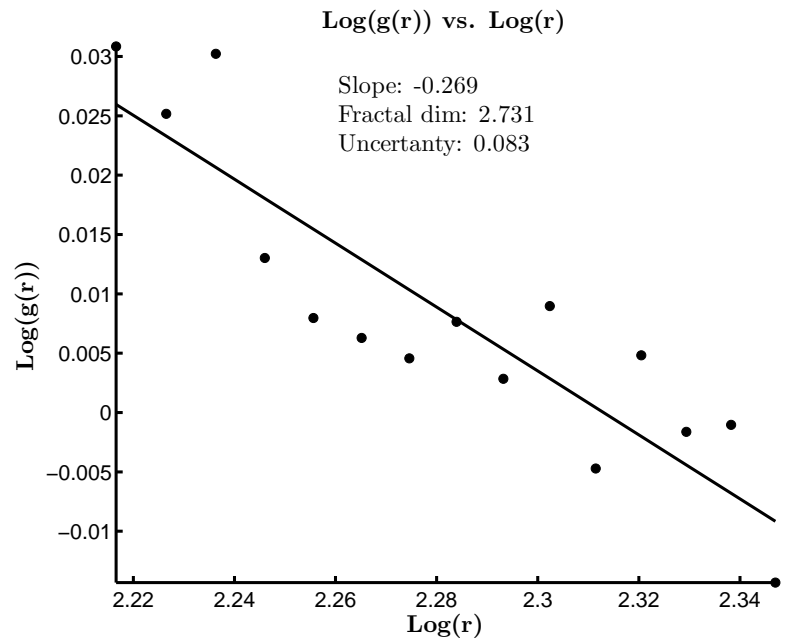

(b)

Figure B.5: Pair Distribution Function and fractal dimension for silica xerogel, $\rho=1.05 \frac{\mathrm{gr}}{\mathrm{cm}^{3}}$.

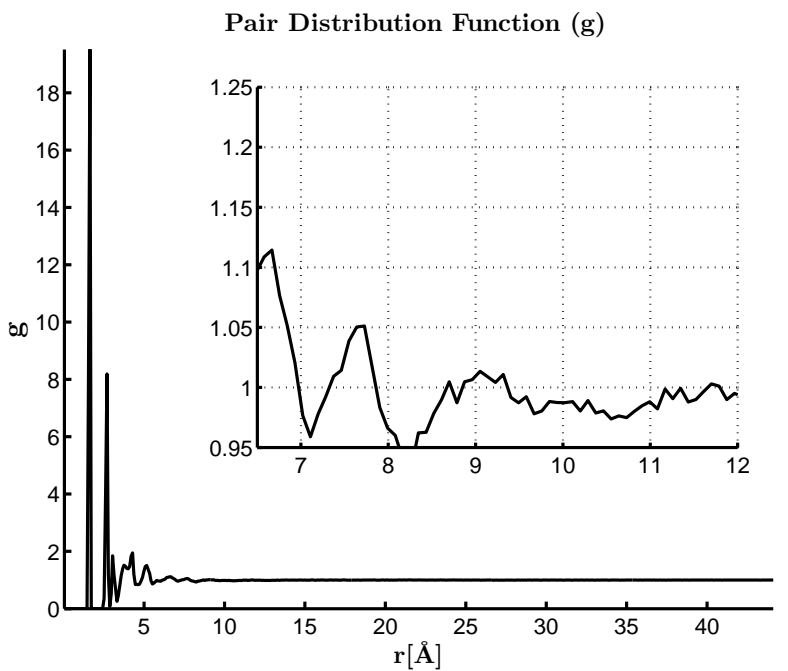

(a)

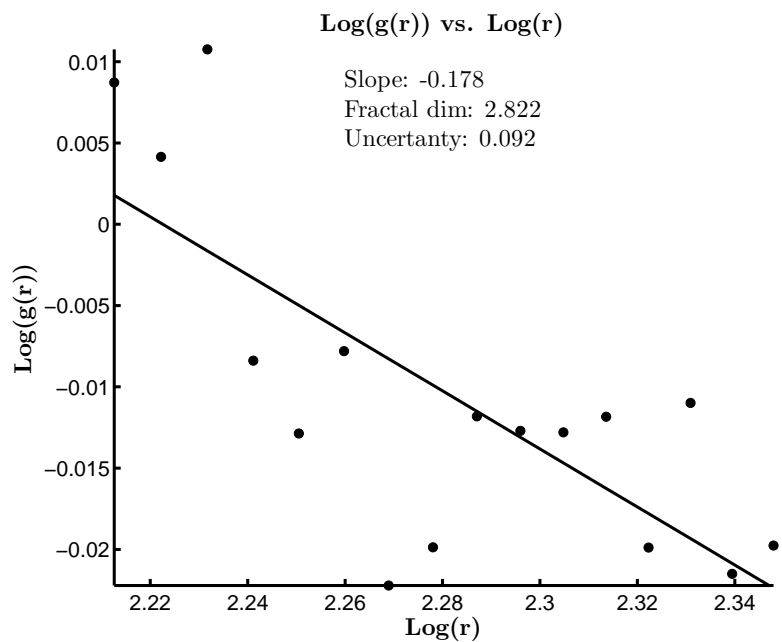

(b)

Figure B.6: Pair Distribution Function and fractal dimension for hybrid xerogel, $\rho=1.05 \frac{\mathrm{gr}}{\mathrm{cm}^{3}}, 0.1 \mathrm{~mol} \%$ $\mathrm{CH}_{3}$ bonded to $\mathrm{Si}$. 


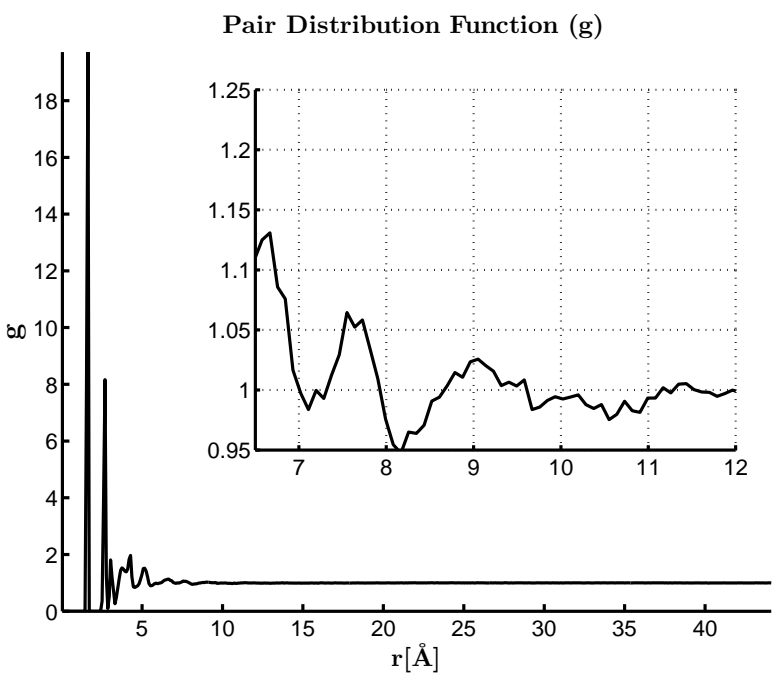

(a)

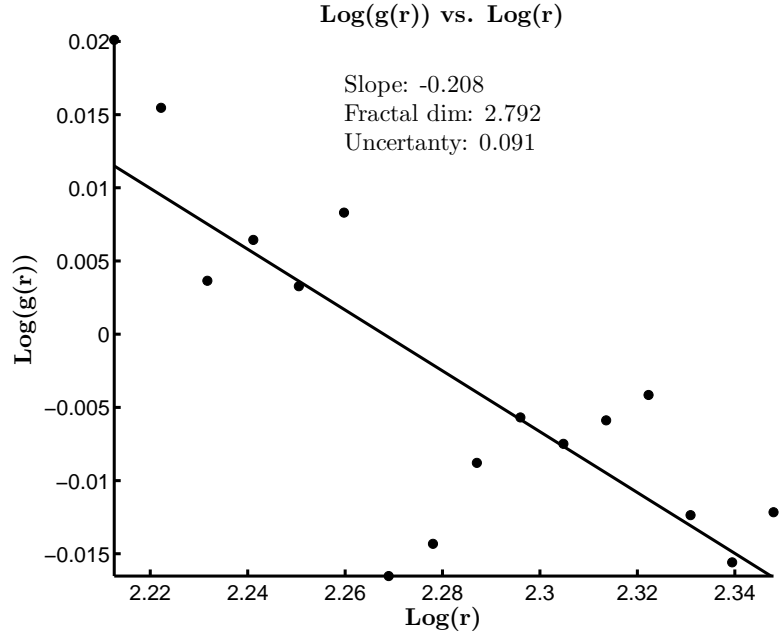

(b)

Figure B.7: Pair Distribution Function and fractal dimension for hybrid xerogel, $\rho=1.05 \frac{g r}{c^{3}}, 0.1 \mathrm{~mol} \%$ $\mathrm{CH}_{3}$ bonded to $\mathrm{O}$.

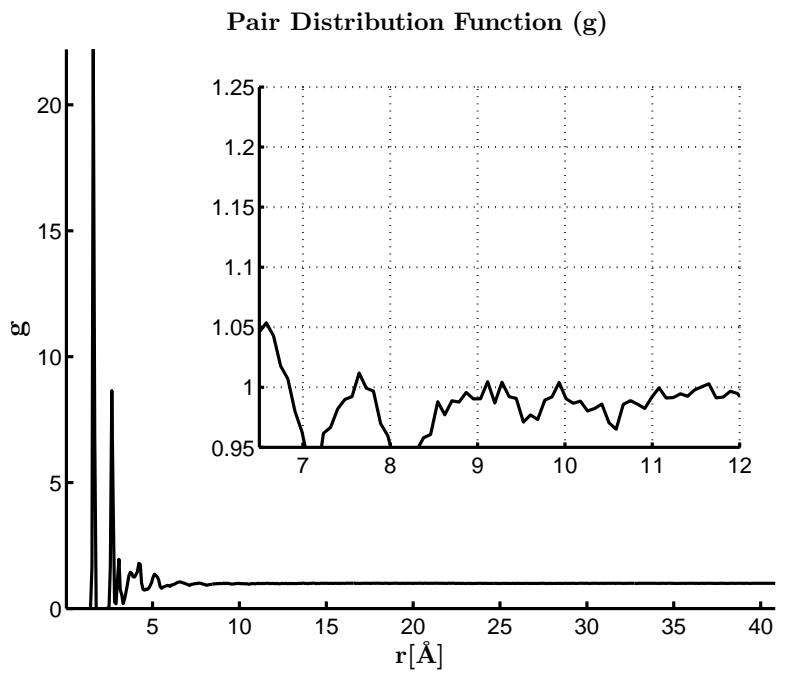

(a)

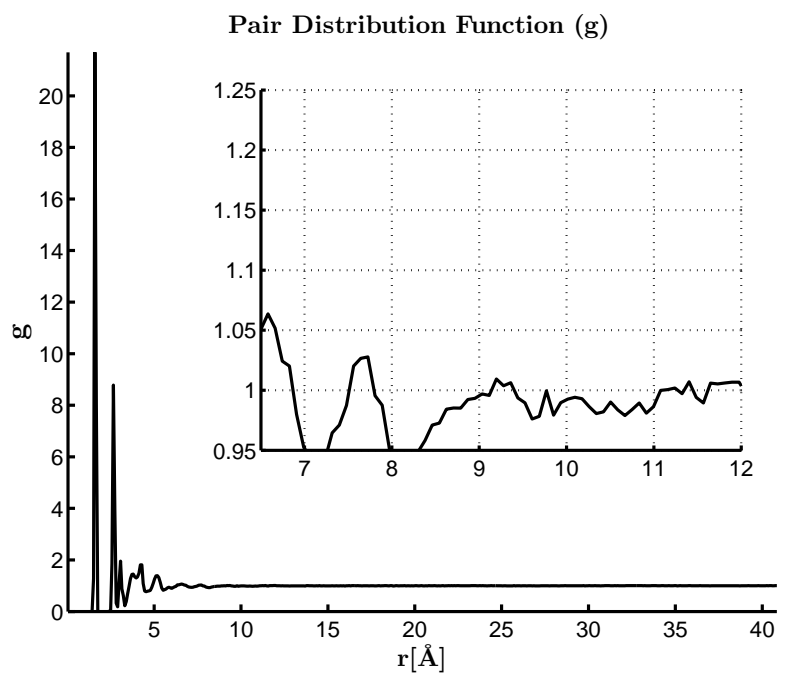

(b)

Figure B.8: Pair Distribution Function hybrid aerogel, $\rho=1.05 \frac{g}{\mathrm{~cm}^{3}}, 0.4 \mathrm{~mol} \% \mathrm{CH}_{3}$ content. (a) $\mathrm{CH}_{3}$ bonded to $\mathrm{Si}$, (b) $\mathrm{CH}_{3}$ bonded to $\mathrm{O}$ 


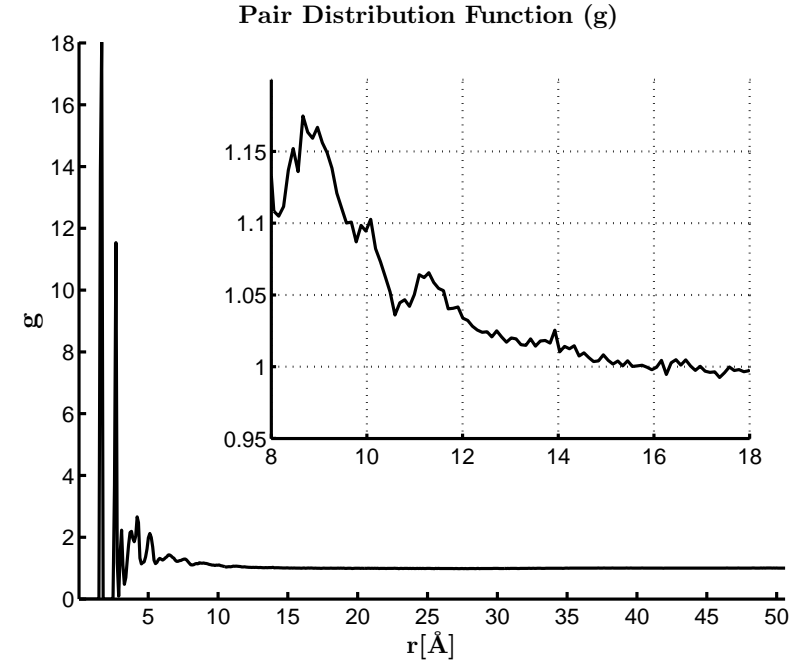

(a)

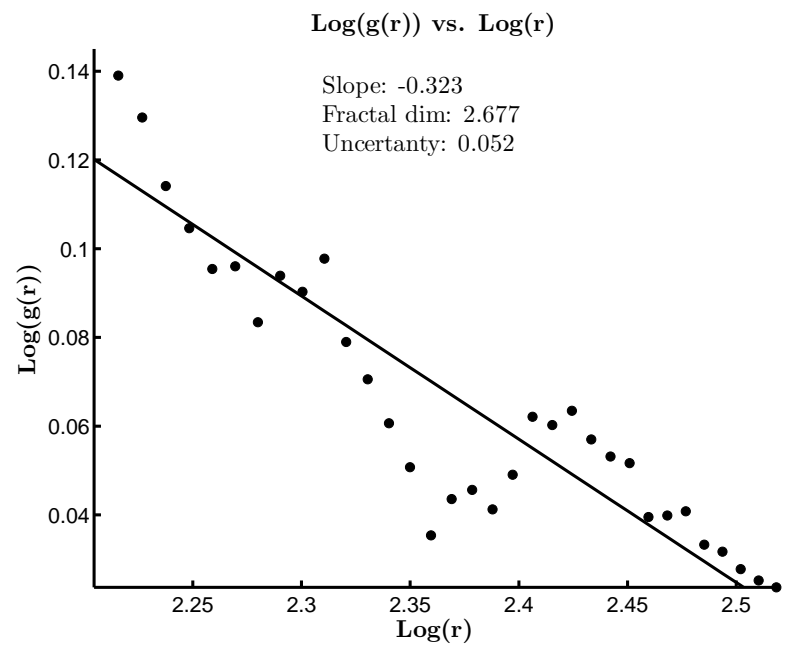

(b)

Figure B.9: Pair Distribution Function and fractal dimension for silica aerogel, $\rho=0.77 \frac{\mathrm{gr}}{\mathrm{cm}^{3}}$.

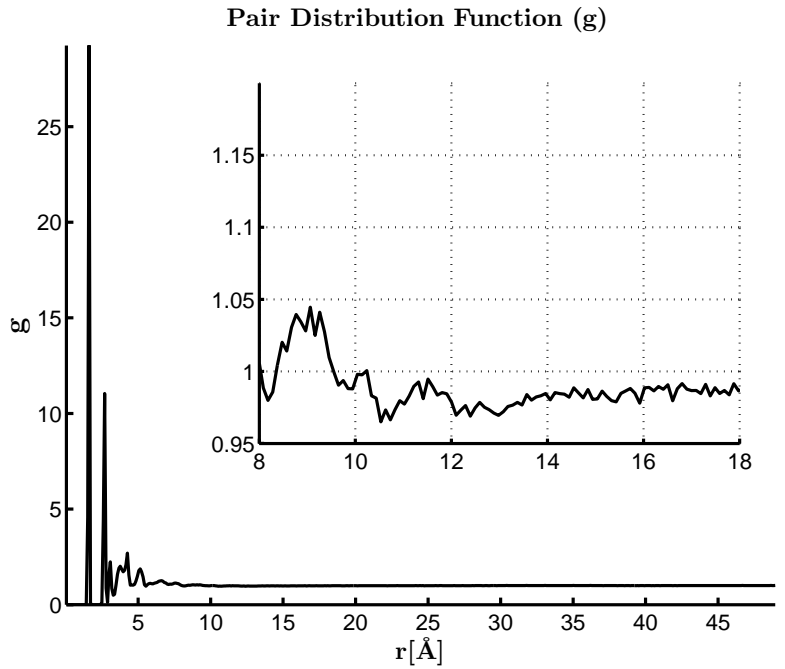

(a)

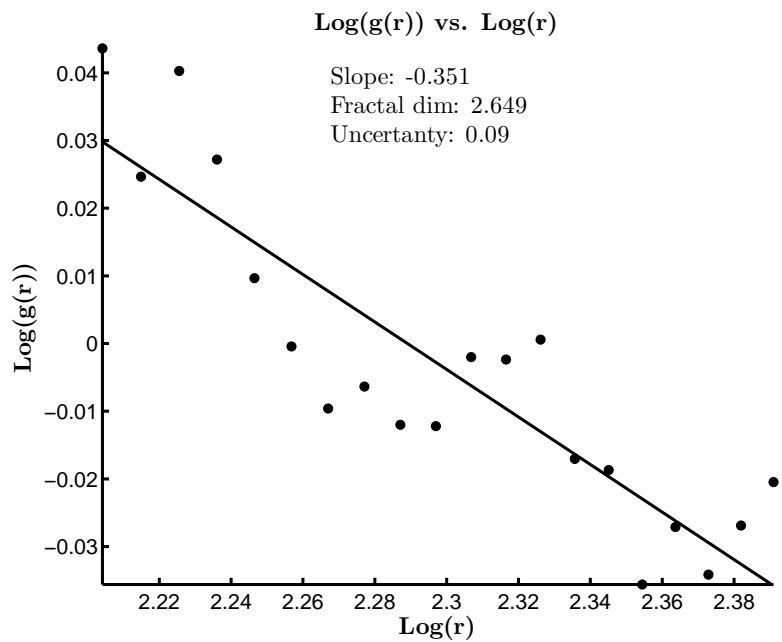

(b)

Figure B.10: Pair Distribution Function and fractal dimension for hybrid xerogel, $\rho=0.77 \frac{\mathrm{gr}}{\mathrm{cm}^{3}}, 0.1 \mathrm{~mol} \%$ $\mathrm{CH}_{3}$ bonded to $\mathrm{Si}$. 


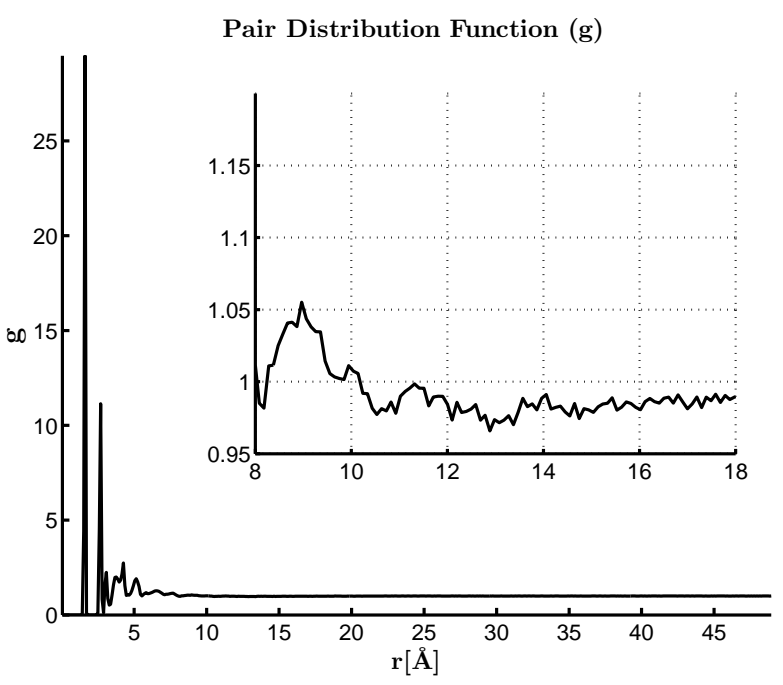

(a)

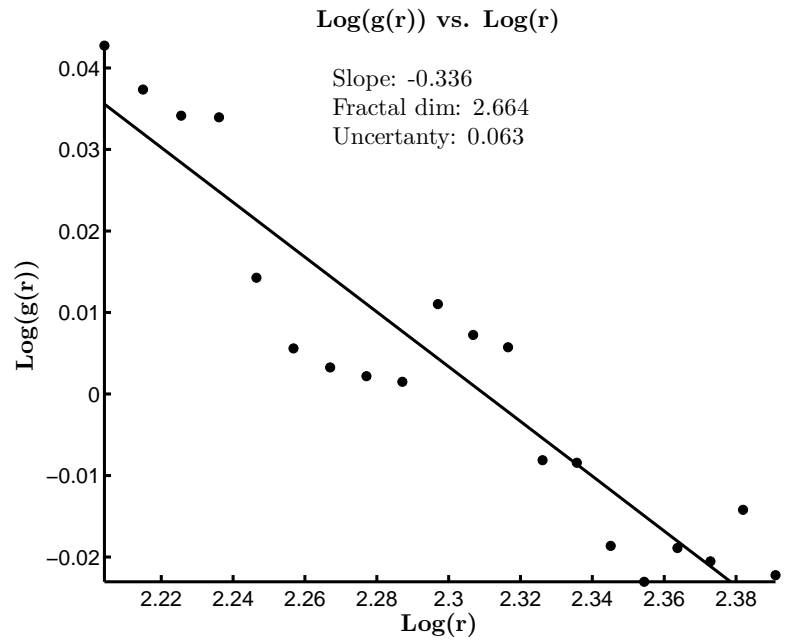

(b)

Figure B.11: Pair Distribution Function and fractal dimension for hybrid xerogel, $\rho=0.77 \frac{g r}{c m^{3}}, 0.1 \mathrm{~mol} \%$ $\mathrm{CH}_{3}$ bonded to $\mathrm{O}$.

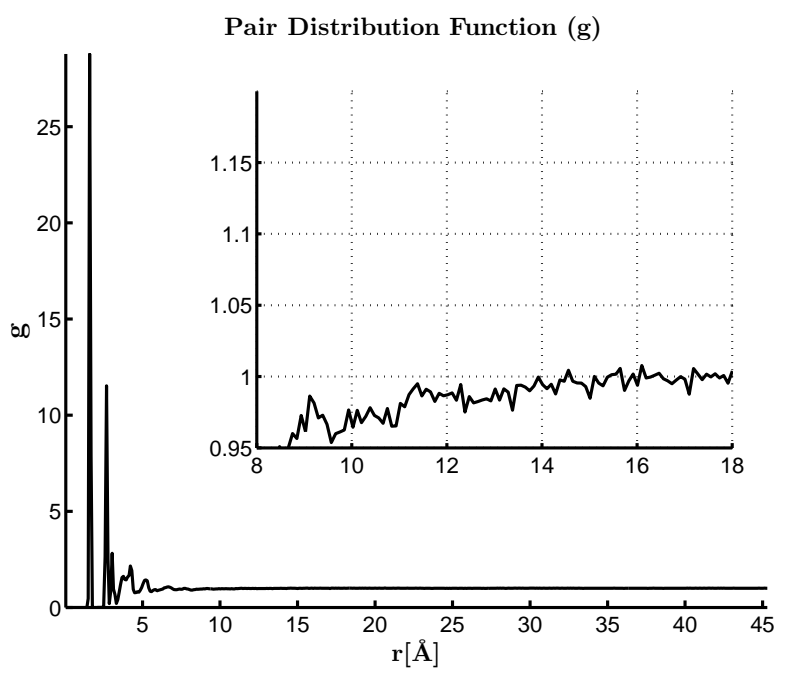

(a)

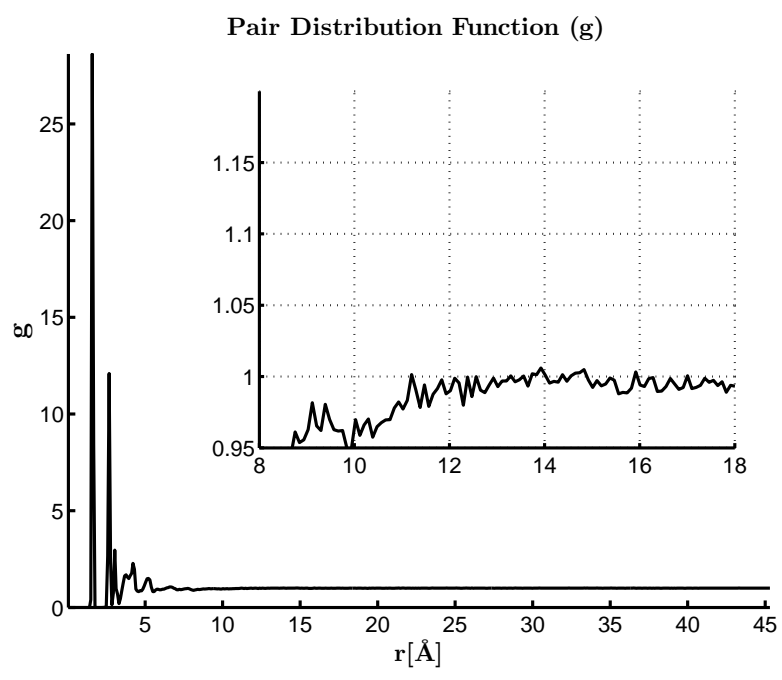

(b)

Figure B.12: Pair Distribution Function hybrid aerogel, $\rho=0.77 \frac{g}{\mathrm{~cm}^{3}}, 0.4 \mathrm{~mol} \% \mathrm{CH}_{3}$ content. (a) $\mathrm{CH}_{3}$ bonded to $\mathrm{Si}$, (b) $\mathrm{CH}_{3}$ bonded to $\mathrm{O}$. 


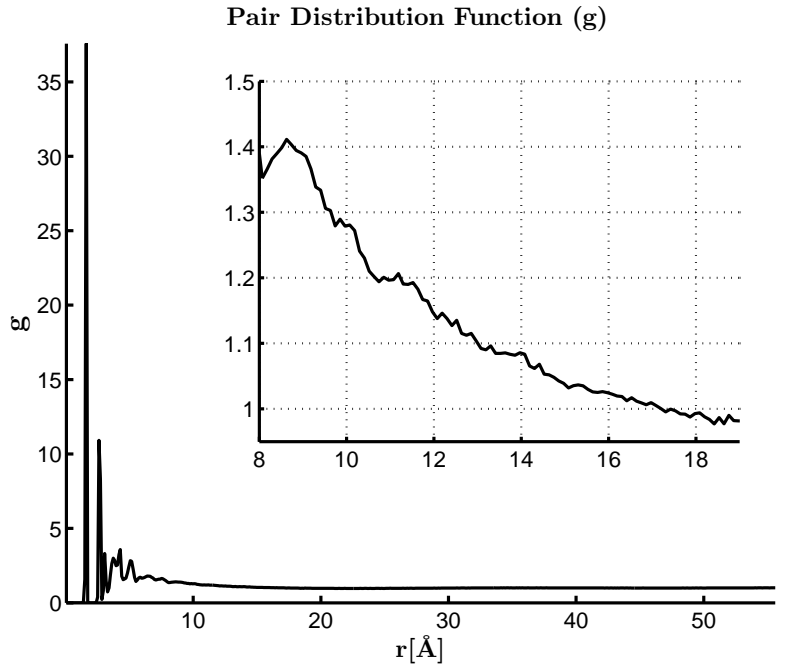

(a)

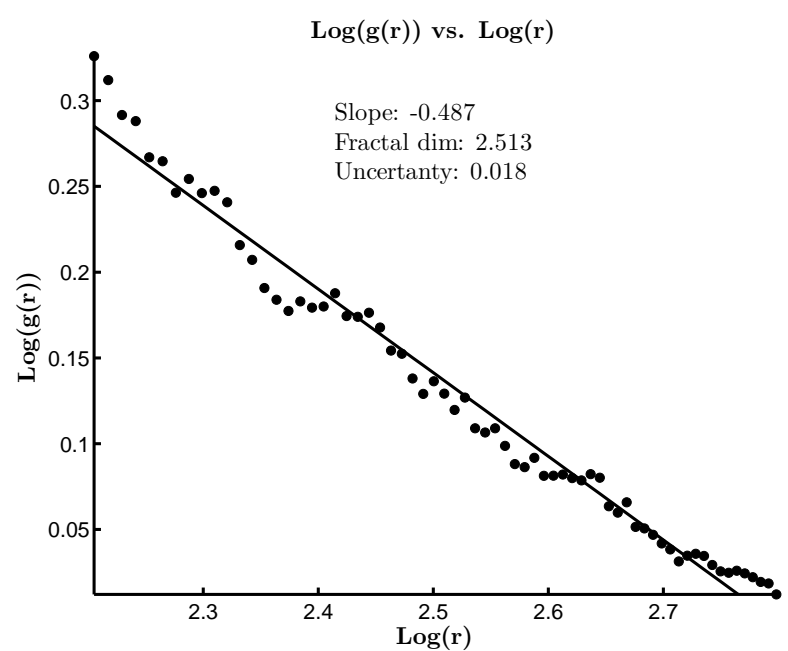

(b)

Figure B.13: Pair Distribution Function and fractal dimension for silica aerogel, $\rho=0.58 \frac{\mathrm{gr}}{\mathrm{cm}^{3}}$.

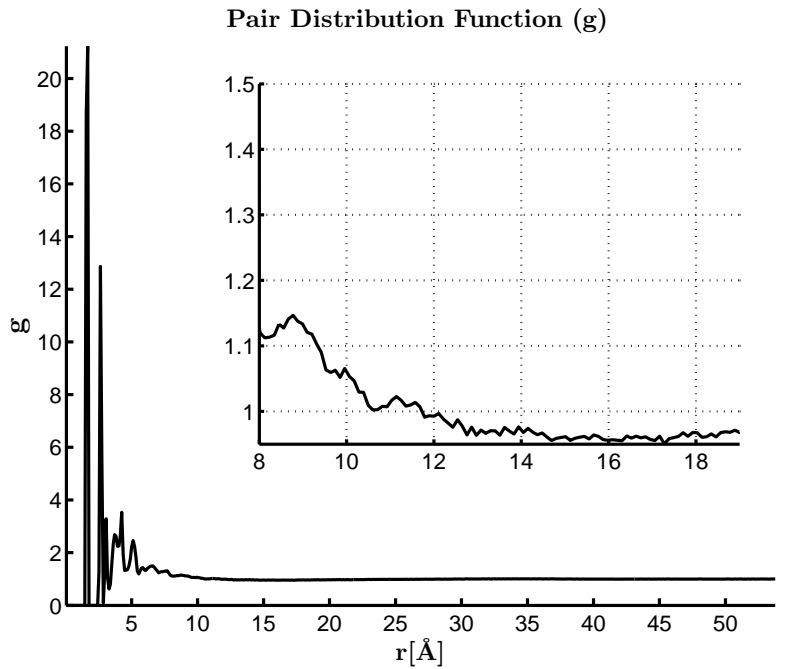

(a)

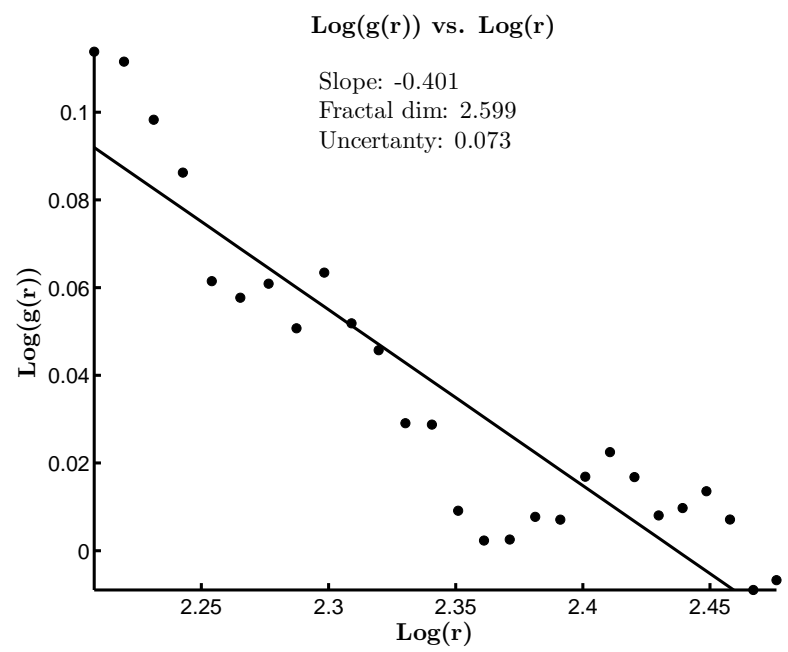

(b)

Figure B.14: Pair Distribution Function and fractal dimension for hybrid xerogel, $\rho=0.58 \frac{g r}{c m^{3}}, 0.1 \mathrm{~mol} \%$ $\mathrm{CH}_{3}$ bonded to $\mathrm{Si}$. 


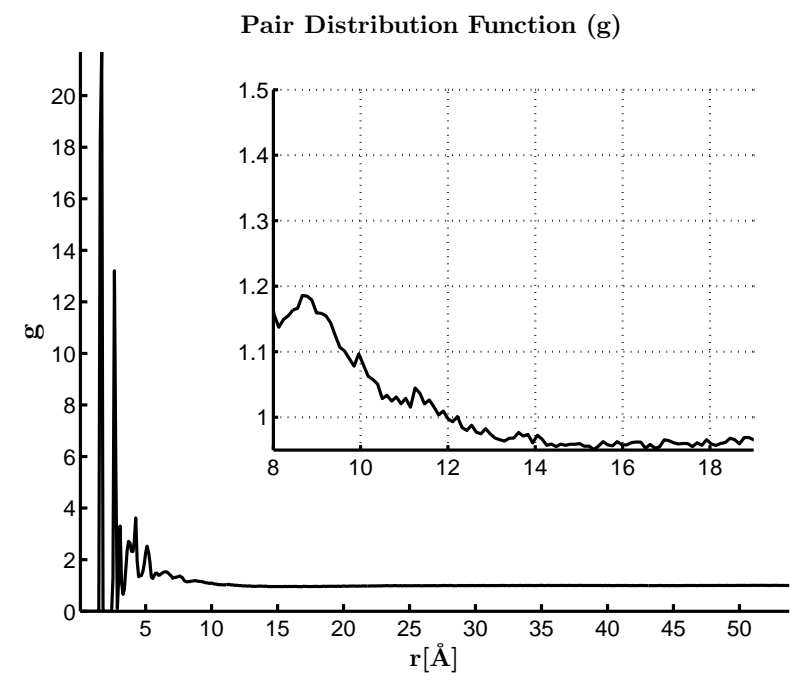

(a)

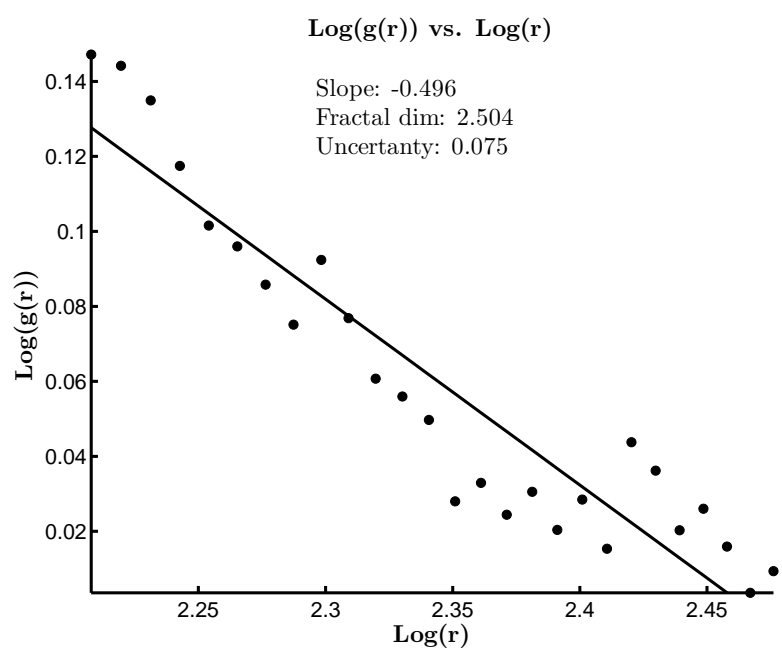

(b)

Figure B.15: Pair Distribution Function and fractal dimension for hybrid xerogel, $\rho=0.58 \frac{g r}{\mathrm{~cm}^{3}}, 0.1 \mathrm{~mol} \%$ $\mathrm{CH}_{3}$ bonded to $\mathrm{O}$.

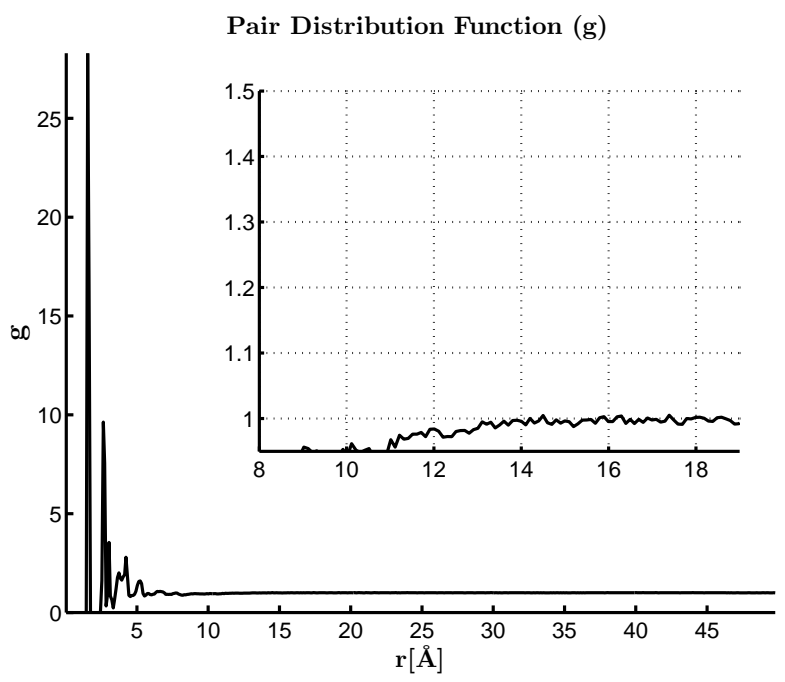

(a)

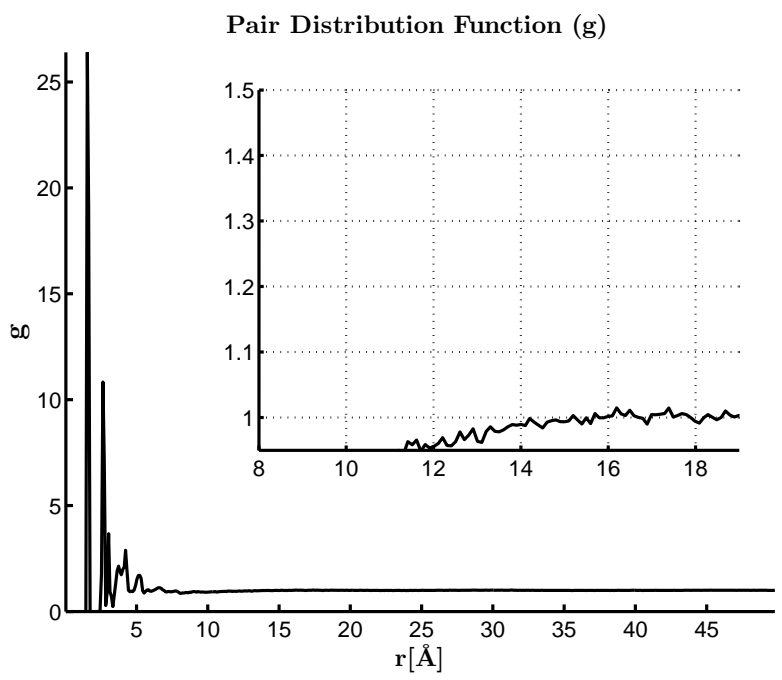

(b)

Figure B.16: Pair Distribution Function hybrid aerogel, $\rho=0.58 \frac{g}{\mathrm{~cm}^{3}}, 0.4 \mathrm{~mol} \% \mathrm{CH}_{3}$ content. (a) $\mathrm{CH}_{3}$ bonded to $\mathrm{Si}$, (b) $\mathrm{CH}_{3}$ bonded to $\mathrm{O}$. 


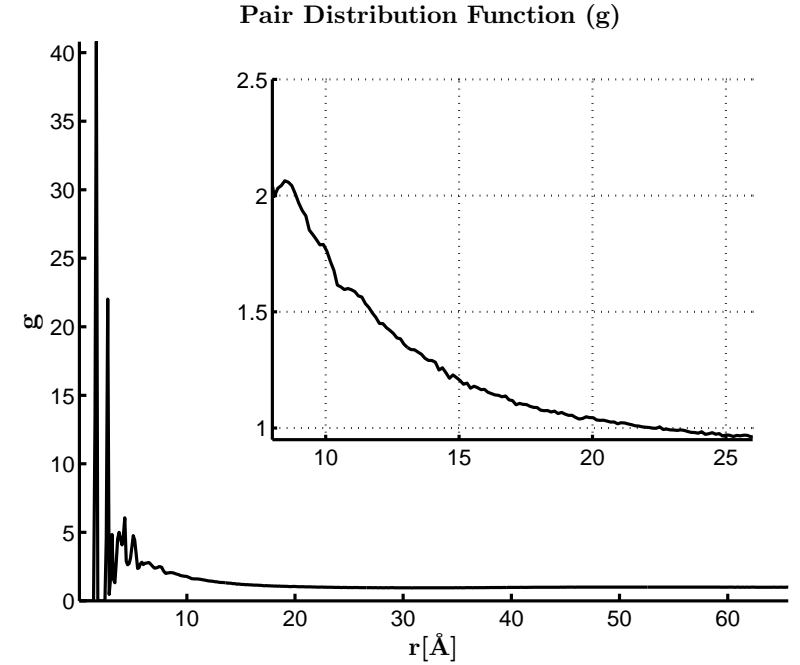

(a)

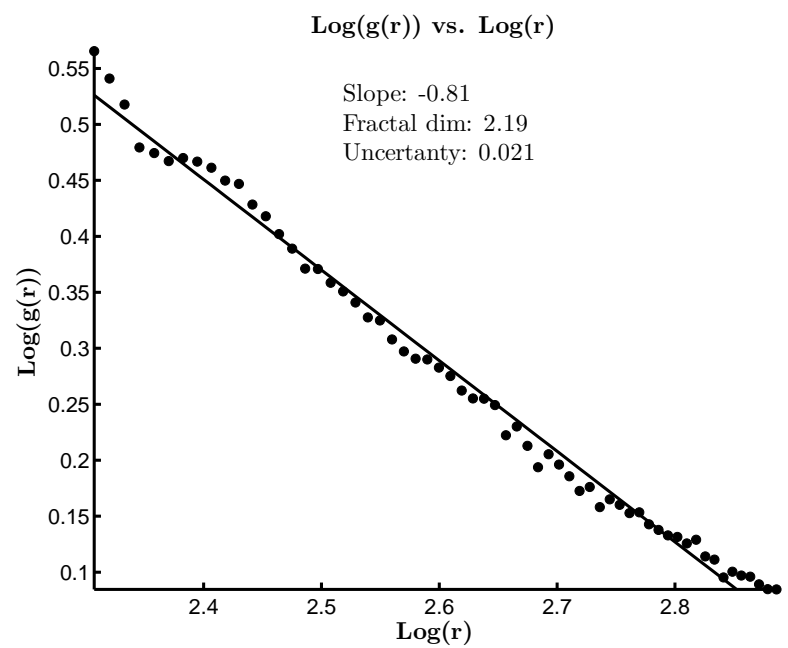

(b)

Figure B.17: Pair Distribution Function and fractal dimension for silica aerogel, $\rho=0.35 \frac{\mathrm{gr}}{\mathrm{cm}^{3}}$.

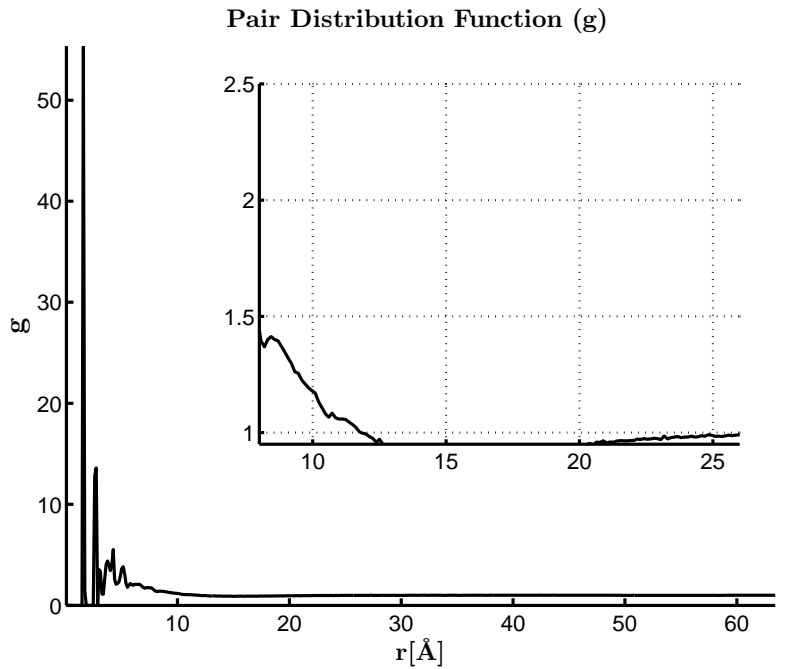

(a)

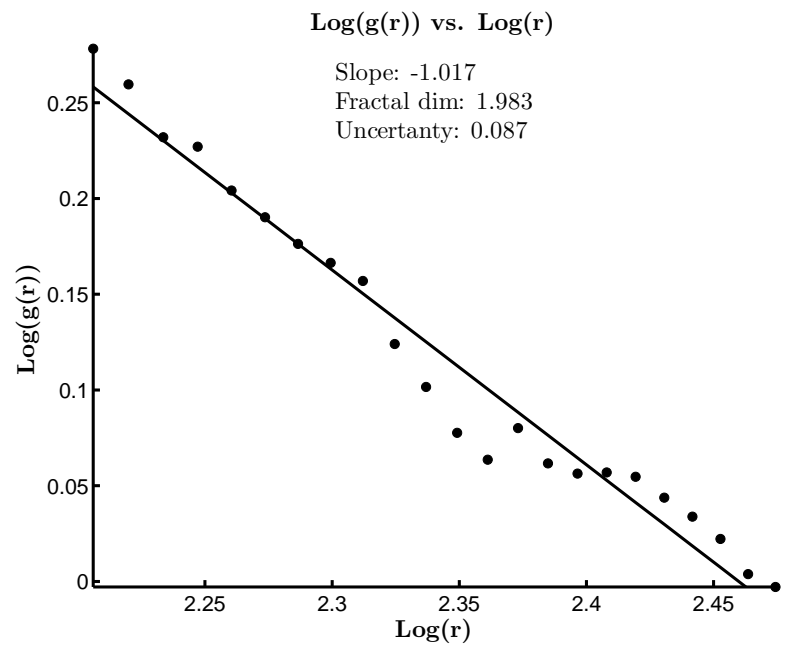

(b)

Figure B.18: Pair Distribution Function and fractal dimension for hybrid xerogel, $\rho=0.35 \frac{g r}{c m^{3}}, 0.1 \mathrm{~mol} \%$ $\mathrm{CH}_{3}$ bonded to $\mathrm{Si}$. 


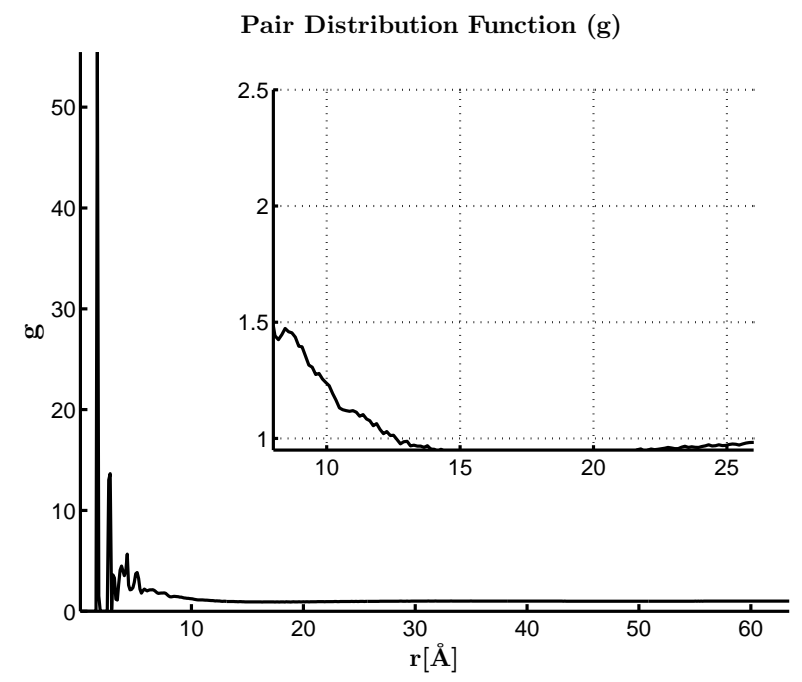

(a)

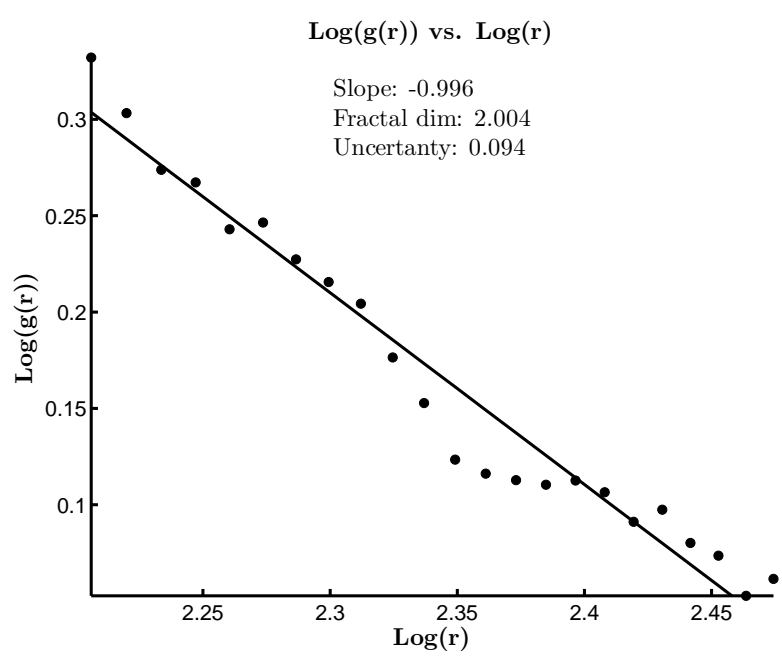

(b)

Figure B.19: Pair Distribution Function and fractal dimension for hybrid xerogel, $\rho=0.35 \frac{g r}{\mathrm{~cm}^{3}}, 0.1 \mathrm{~mol} \%$ $\mathrm{CH}_{3}$ bonded to $\mathrm{O}$.

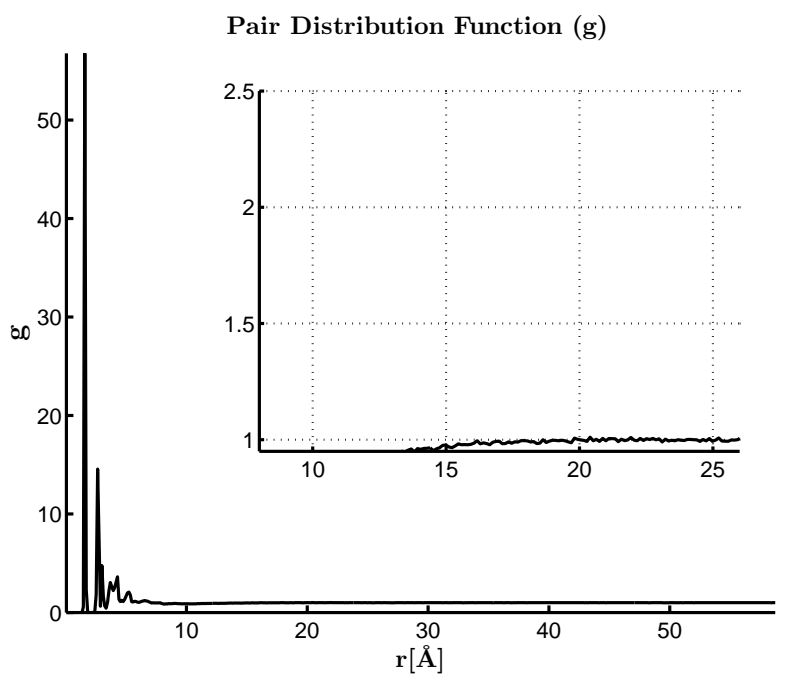

(a)

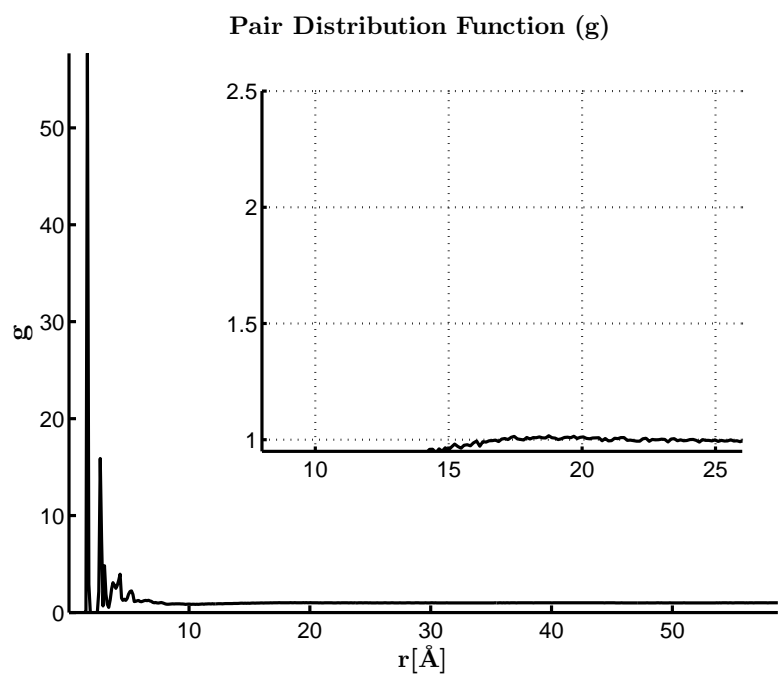

(b)

Figure B.20: Pair Distribution Function hybrid aerogel, $\rho=0.35 \frac{\mathrm{g}}{\mathrm{cm}^{3}}, 0.4 \mathrm{~mol} \% \mathrm{CH}_{3}$ content. (a) $\mathrm{CH}_{3}$ bonded to $\mathrm{Si}$, (b) $\mathrm{CH}_{3}$ bonded to $\mathrm{O}$. 


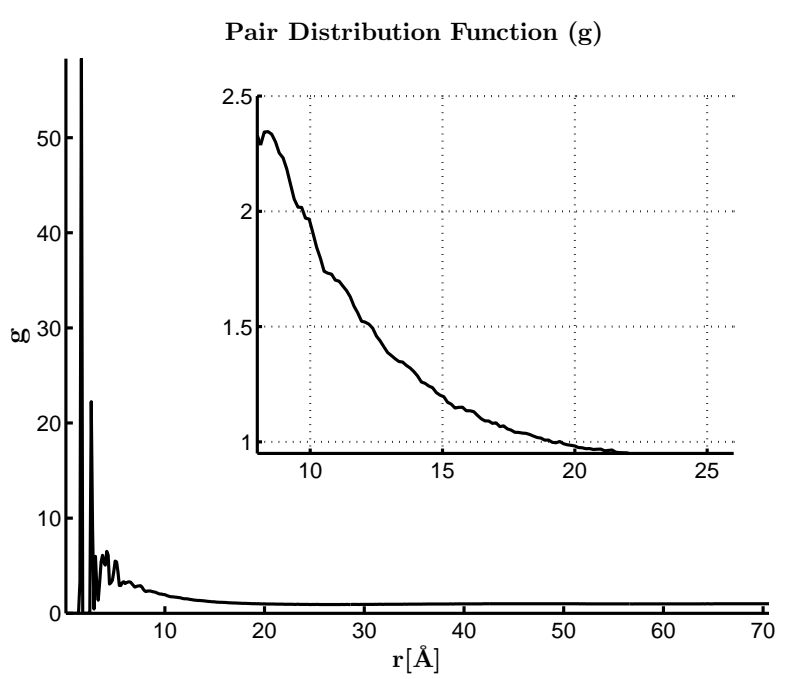

(a)

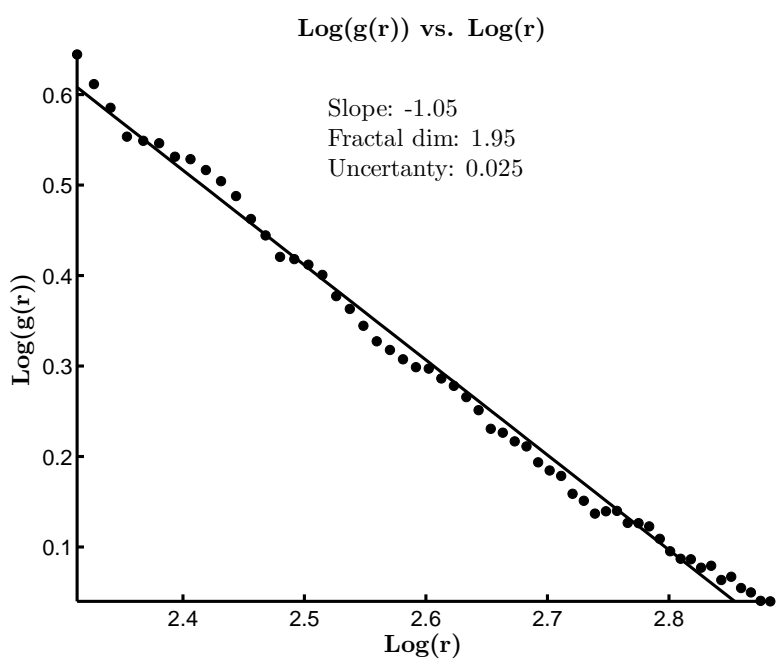

(b)

Figure B.21: Pair Distribution Function and fractal dimension for silica aerogel, $\rho=0.28 \frac{\mathrm{gr}}{\mathrm{cm}^{3}}$.

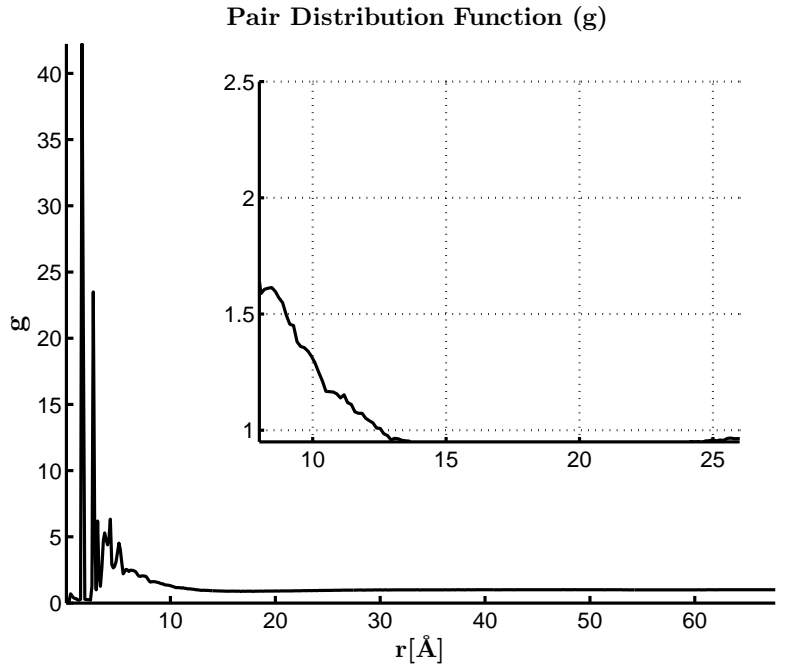

(a)

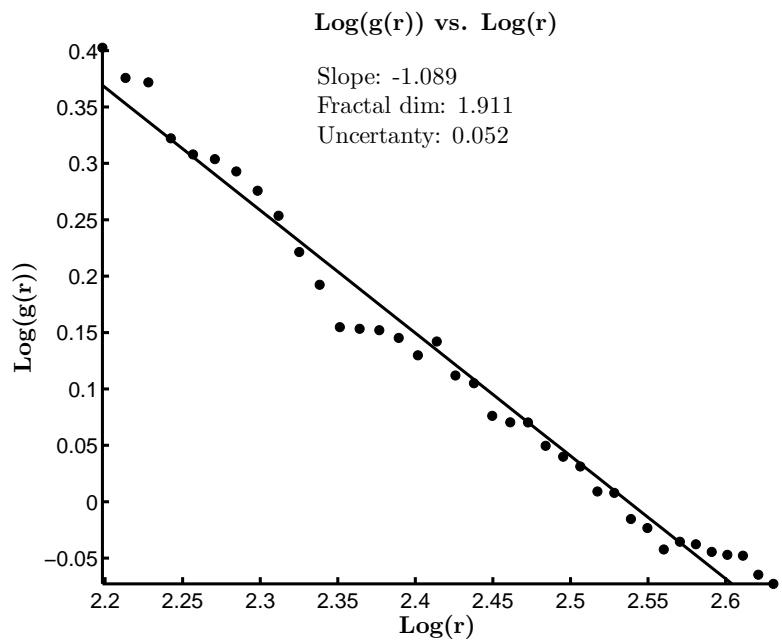

(b)

Figure B.22: Pair Distribution Function and fractal dimension for hybrid xerogel, $\rho=0.28 \frac{\mathrm{gr}}{\mathrm{cm}^{3}}, 0.1 \mathrm{~mol} \%$ $\mathrm{CH}_{3}$ bonded to $\mathrm{Si}$. 


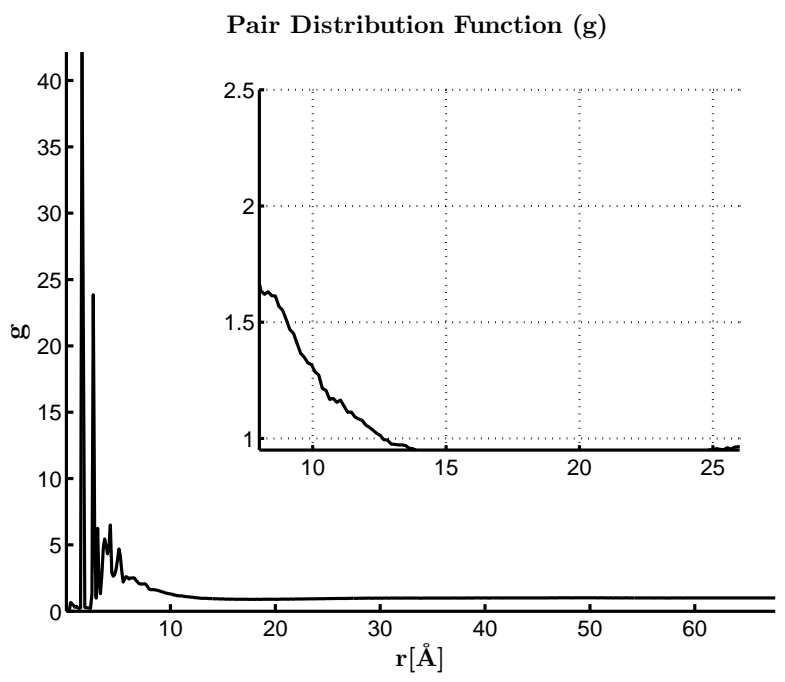

(a)

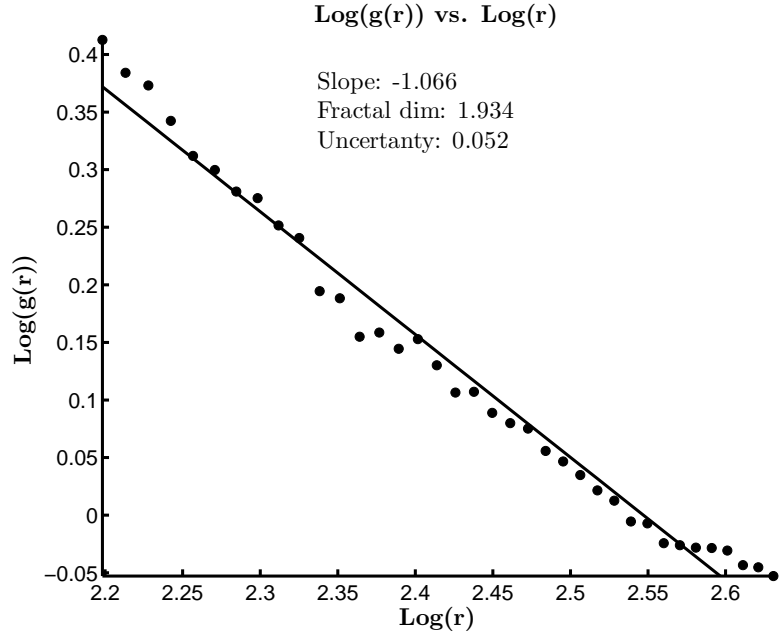

(b)

Figure B.23: Pair Distribution Function and fractal dimension for hybrid xerogel, $\rho=0.28 \frac{g r}{\mathrm{~cm}^{3}}, 0.1 \mathrm{~mol} \%$ $\mathrm{CH}_{3}$ bonded to $\mathrm{O}$.

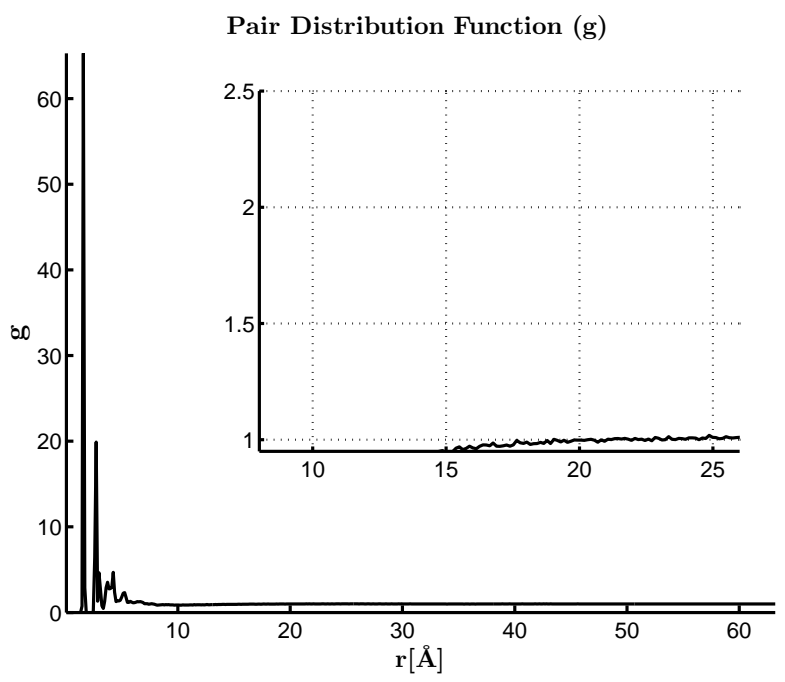

(a)

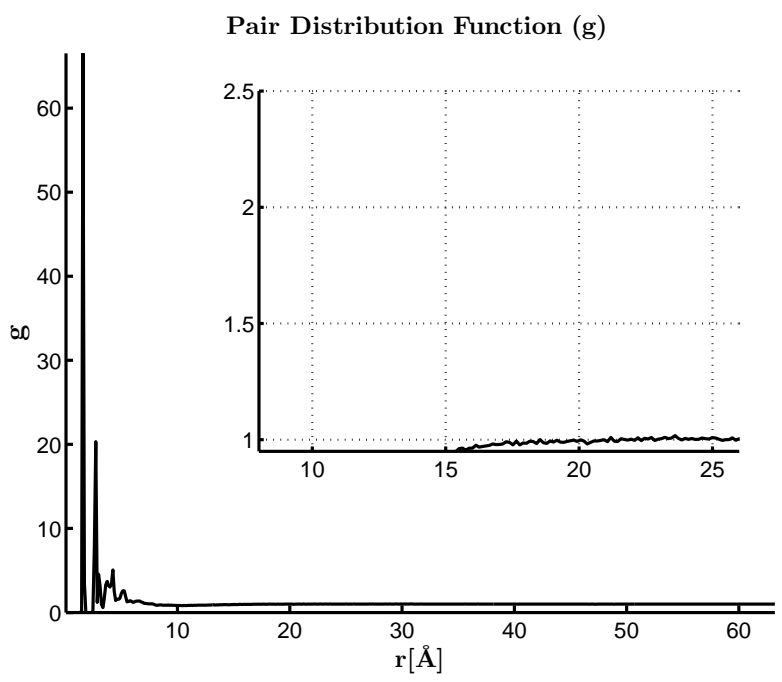

(b)

Figure B.24: Pair Distribution Function hybrid aerogel, $\rho=0.28 \frac{g}{\mathrm{~cm}^{3}}, 0.4 \mathrm{~mol} \% \mathrm{CH}_{3}$ content. (a) $\mathrm{CH}_{3}$ bonded to $\mathrm{Si}$, (b) $\mathrm{CH}_{3}$ bonded to $\mathrm{O}$. 
Appendix C

Additional Figures: Stress vs. Strain 


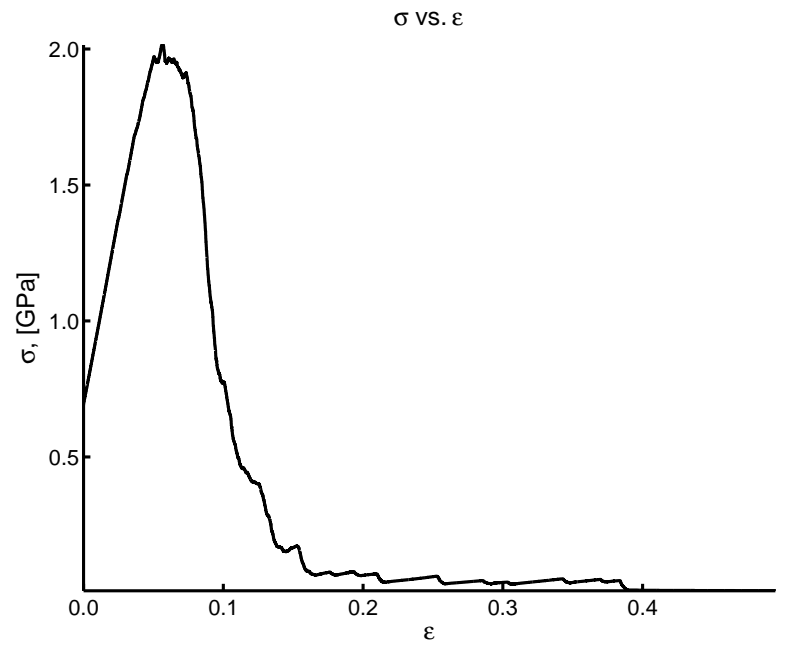

(a)

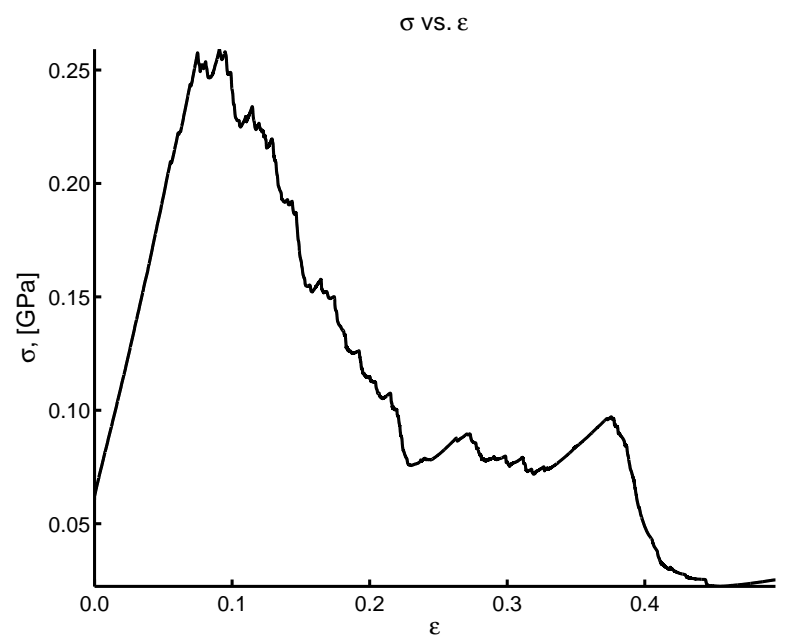

(c)

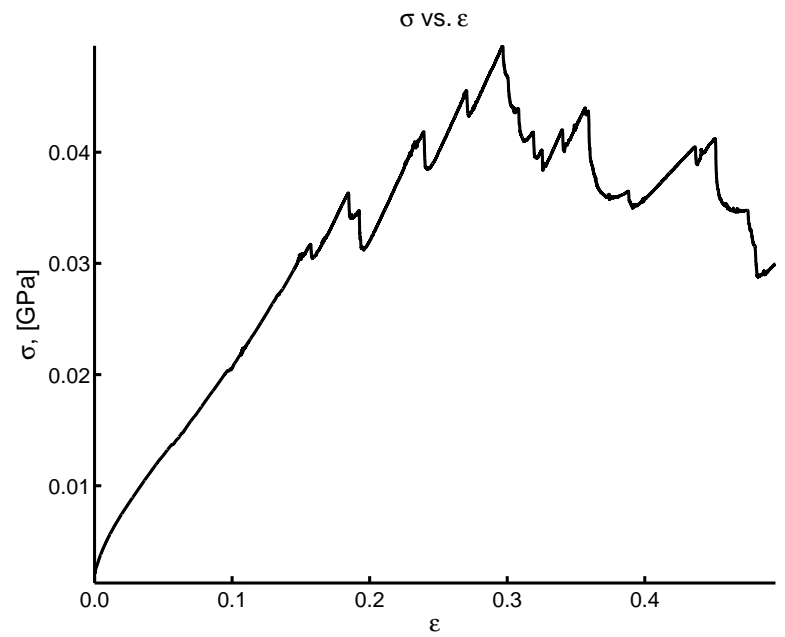

(e)

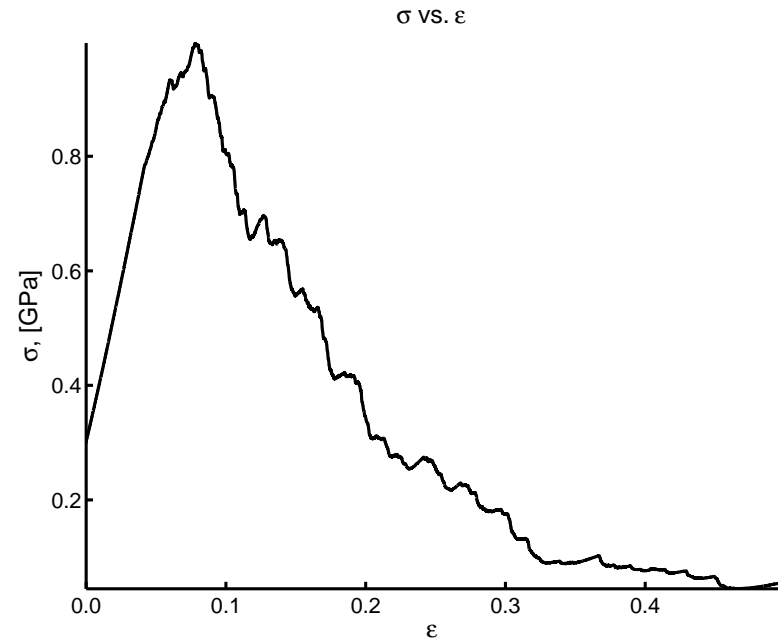

(b)

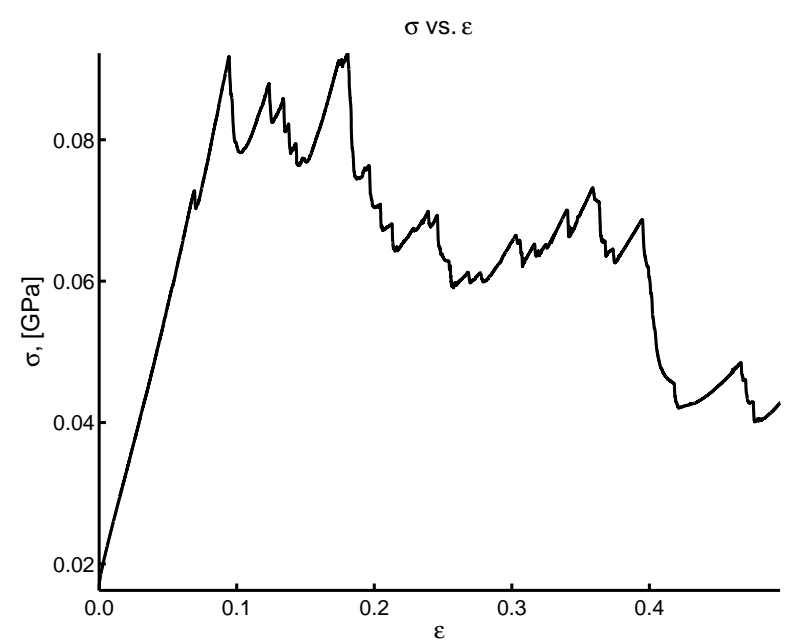

(d)

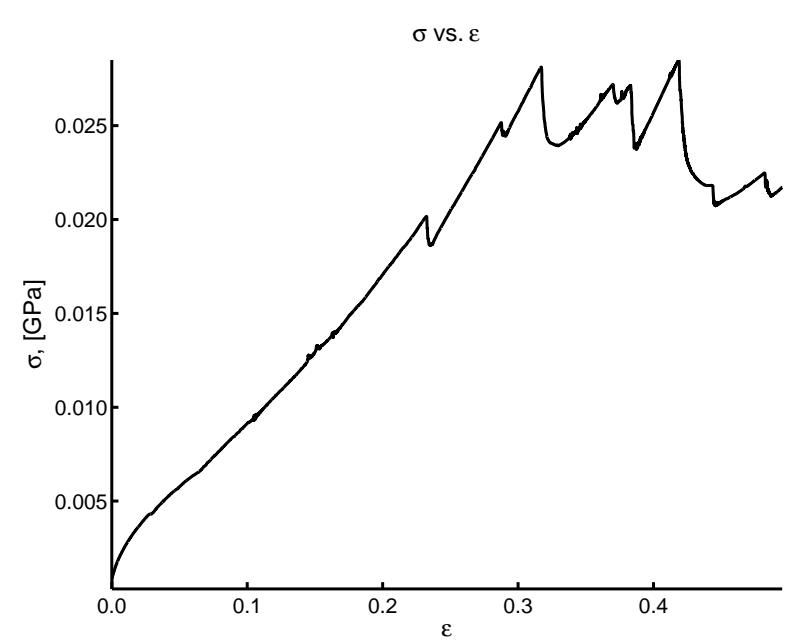

(f)

Figure C.1: Stress vs. Strain plots for silica aerogels and xerogels, $0 \mathrm{~mol} \% \mathrm{CH}_{3}$ content. (a) $\rho=1.49 \frac{g}{\mathrm{~cm}^{3}}$, (b) $\rho=1.05 \frac{g}{c^{3}}$, (c) $\rho=0.58 \frac{g}{\mathrm{~cm}^{3}}$, (d) $\rho=0.45 \frac{g}{\mathrm{~cm}^{3}}$, (e) $\rho=0.35 \frac{g}{\mathrm{~cm}^{3}}$, (f) $\rho=0.28 \frac{g}{\mathrm{~cm}^{3}}$ 


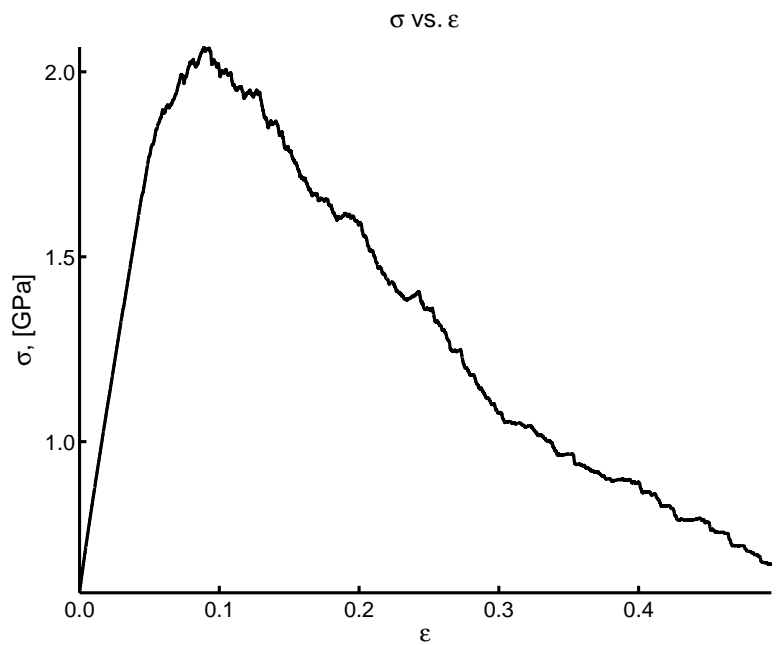

(a)

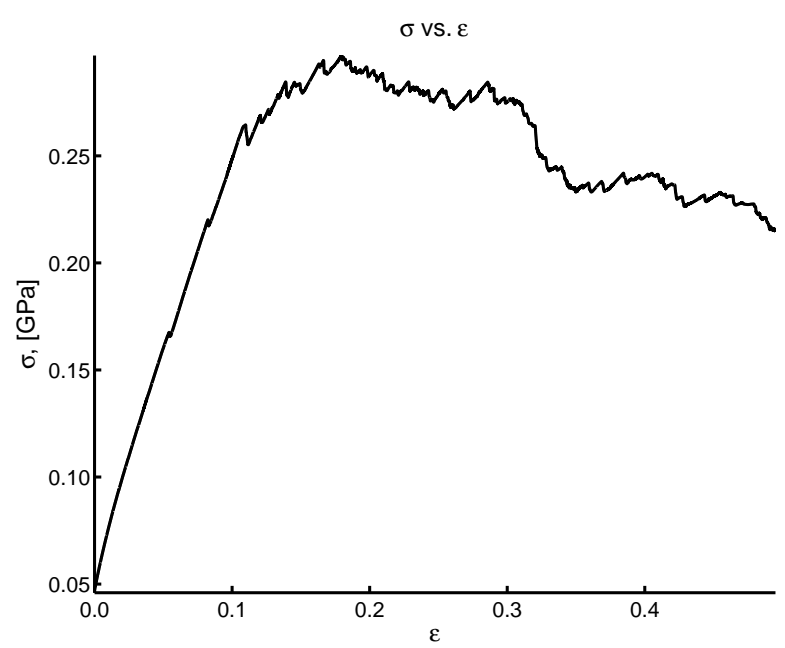

(c)

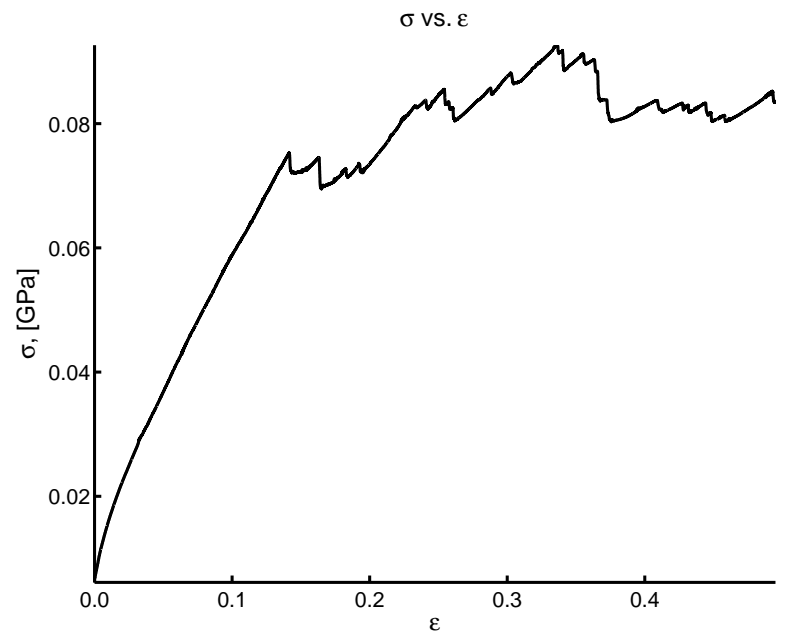

(e)

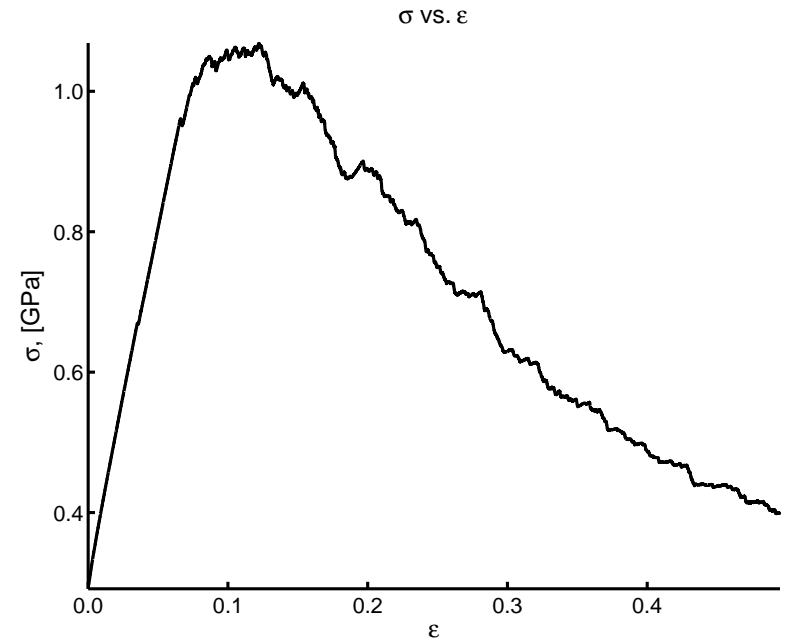

(b)

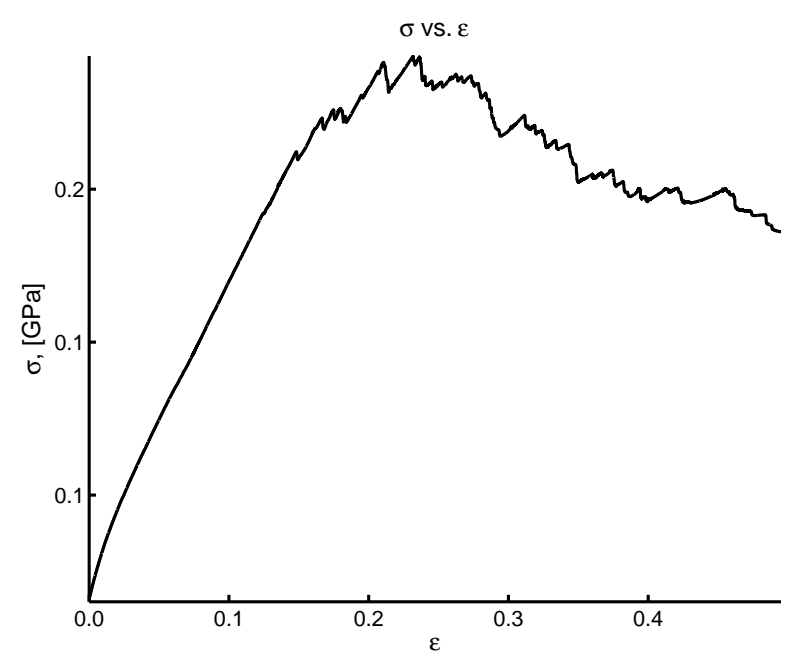

(d)

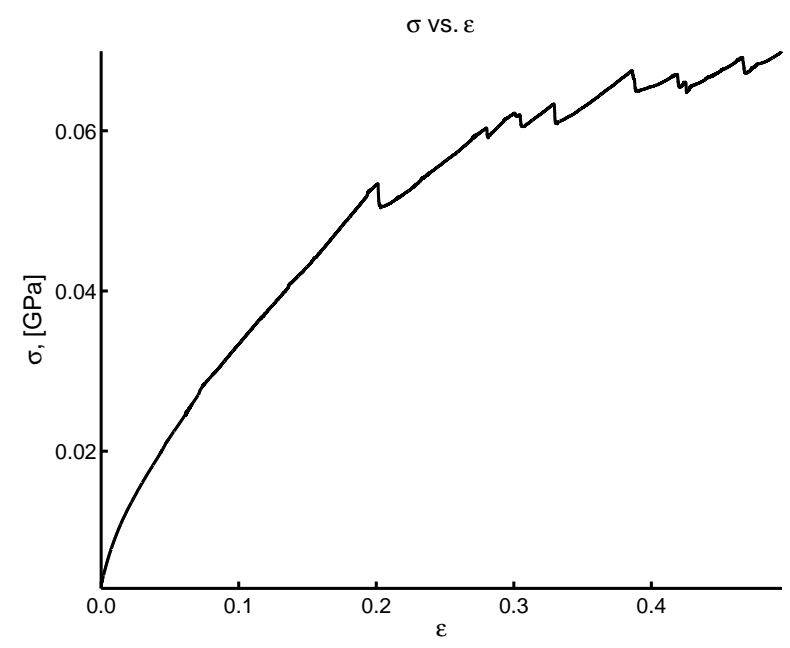

(f)

Figure C.2: Stress vs. Strain plots for silica aerogels and xerogels, $0.1 \mathrm{~mol} \% \mathrm{CH}_{3}$ bonded to Si. (a) $\rho=$ $1.49 \frac{g}{\mathrm{~cm}^{3}}$, (b) $\rho=1.05 \frac{\mathrm{g}}{\mathrm{cm}^{3}}$, (c) $\rho=0.58 \frac{g}{\mathrm{~cm}^{3}}$, (d) $\rho=0.45 \frac{g}{\mathrm{~cm}^{3}}$, (e) $\rho=0.35 \frac{g}{\mathrm{~cm}^{3}}$, (f) $\rho=0.28 \frac{\mathrm{g}}{\mathrm{cm}^{3}}$ 


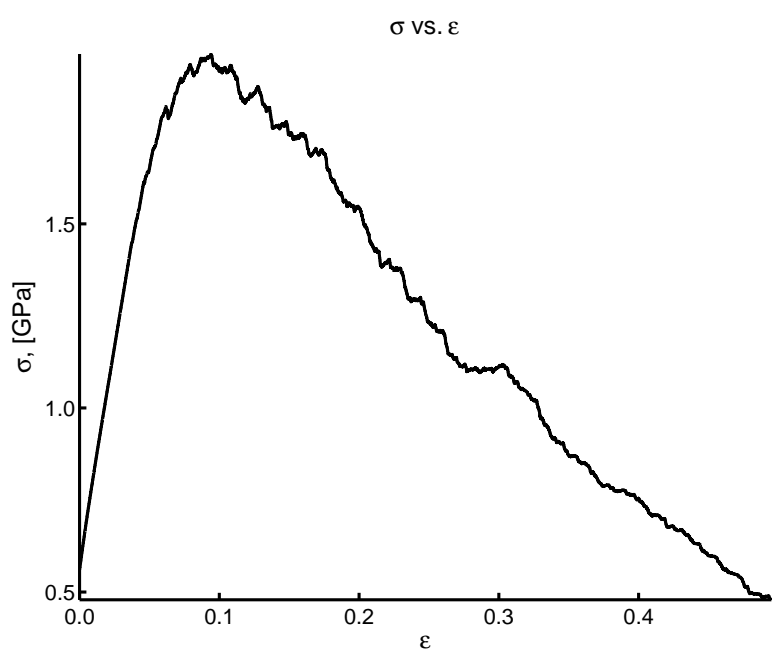

(a)

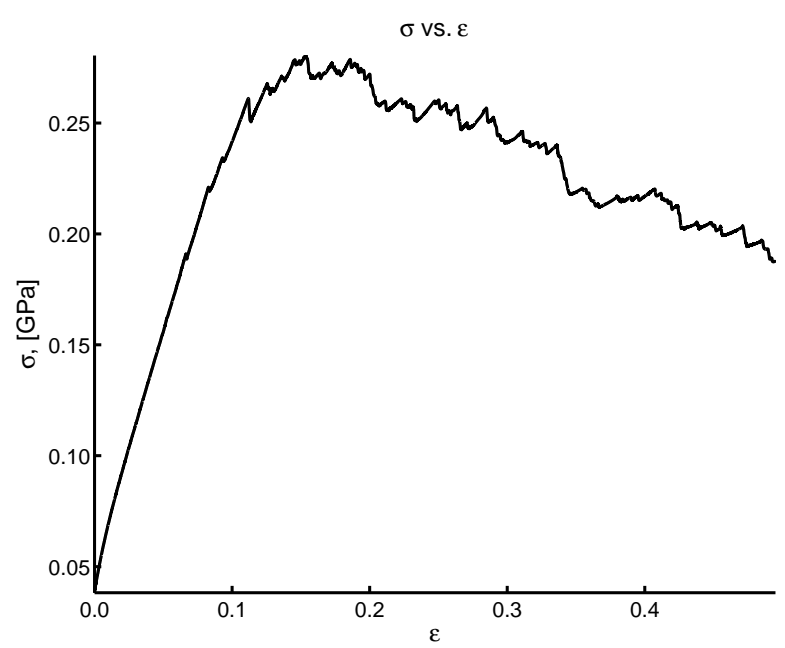

(c)

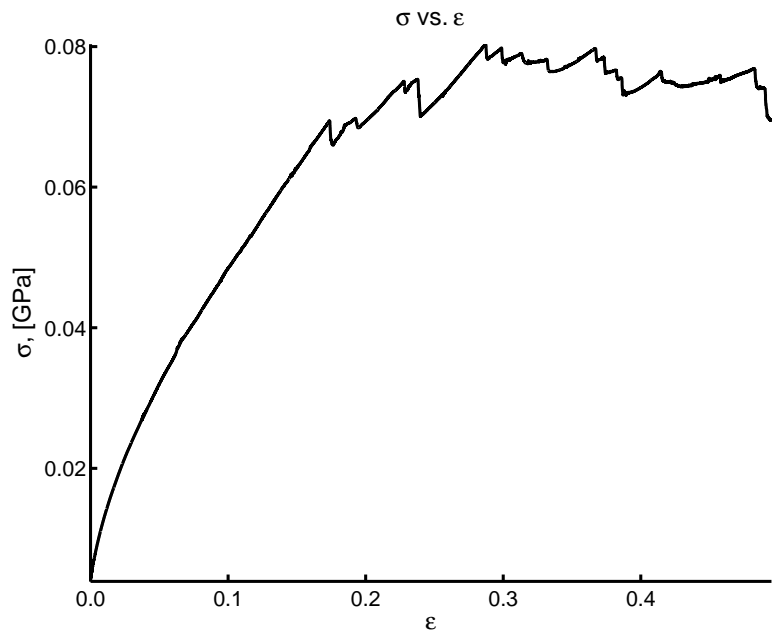

(e)

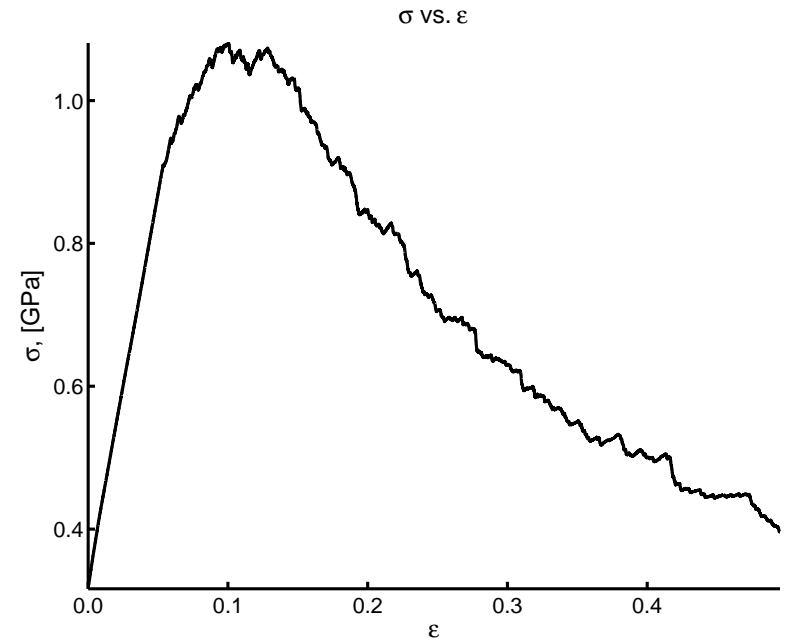

(b)

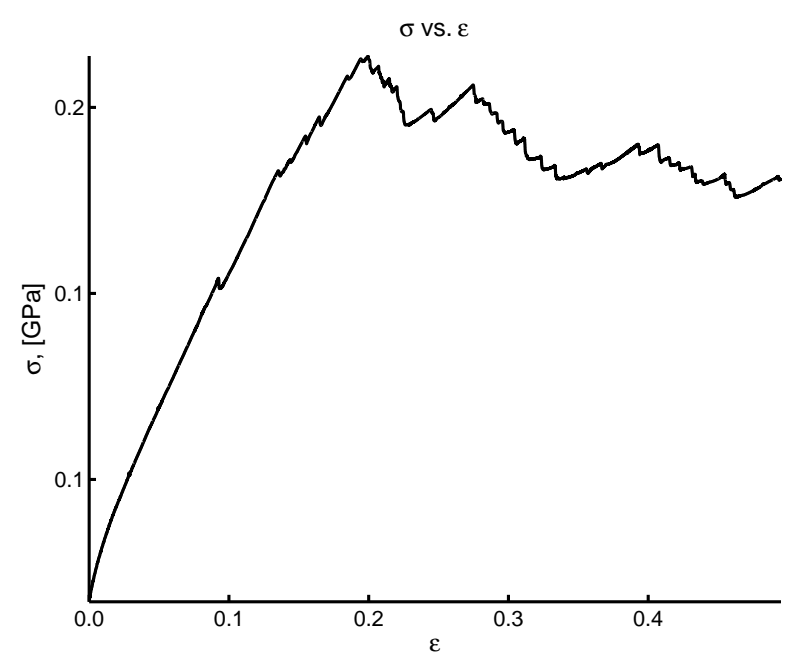

(d)

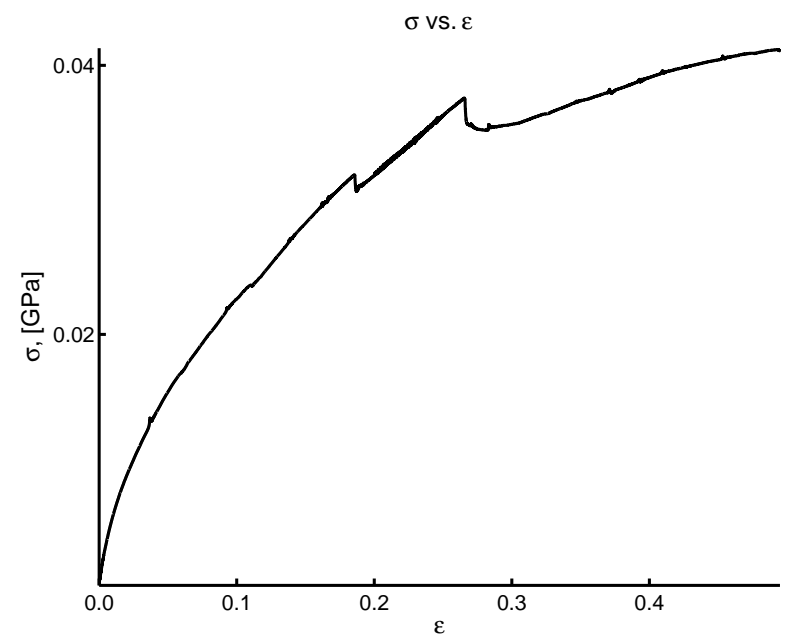

(f)

Figure C.3: Stress vs. Strain plots for silica aerogels and xerogels, $0.1 \mathrm{~mol} \% \mathrm{CH}_{3}$ bonded to O. (a) $\rho=$ $1.49 \frac{g}{\mathrm{~cm}^{3}}$, (b) $\rho=1.05 \frac{\mathrm{g}}{\mathrm{cm}^{3}}$, (c) $\rho=0.58 \frac{\mathrm{g}}{\mathrm{cm}^{3}}$, (d) $\rho=0.45 \frac{\mathrm{g}}{\mathrm{cm}^{3}}$, (e) $\rho=0.35 \frac{\mathrm{g}}{\mathrm{cm}^{3}}$, (f) $\rho=0.28 \frac{\mathrm{g}}{\mathrm{cm}^{3}}$ 


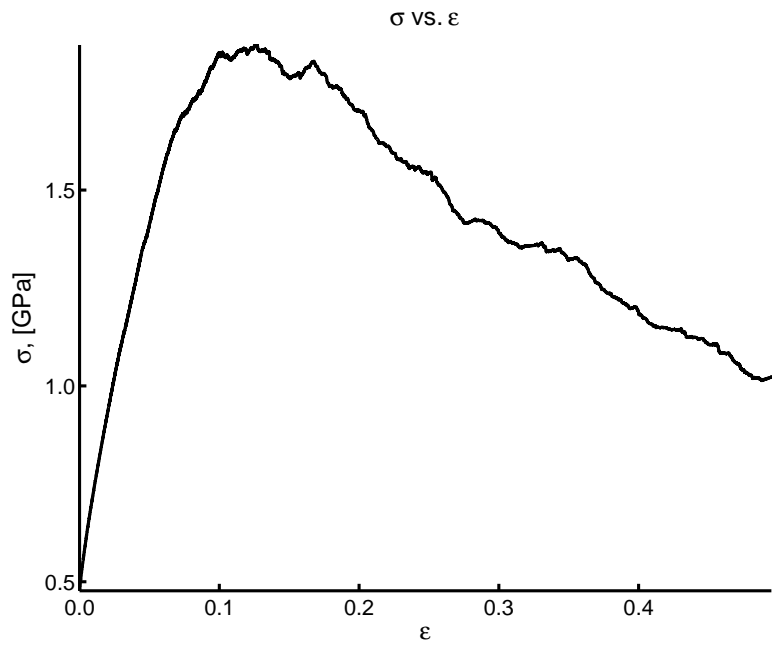

(a)

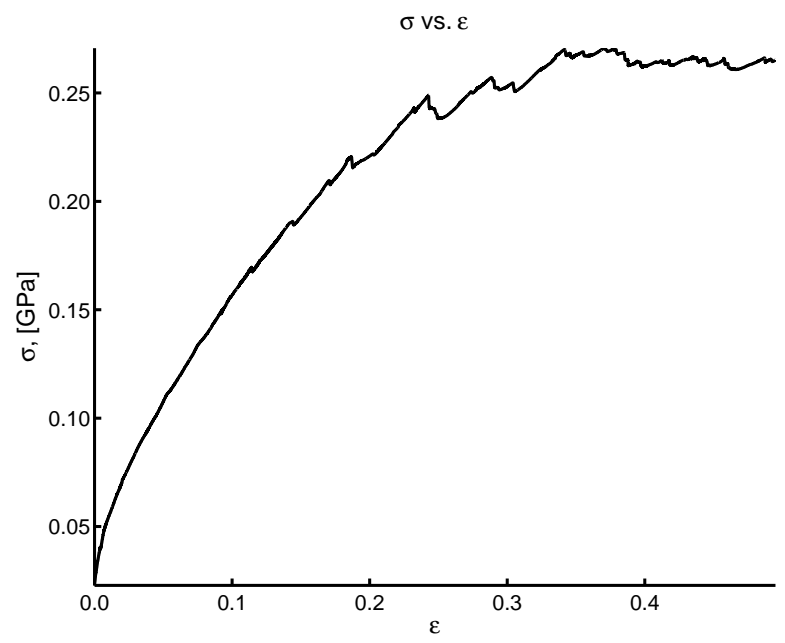

(c)

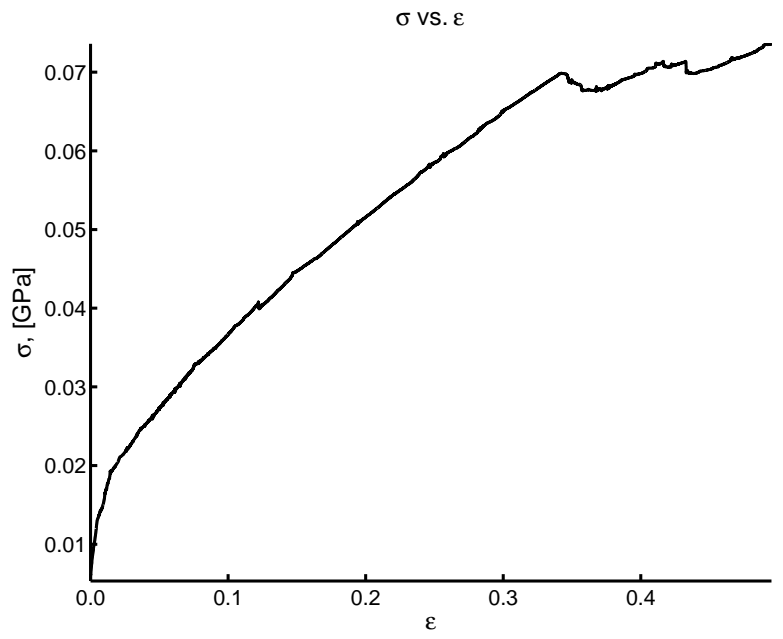

(e)

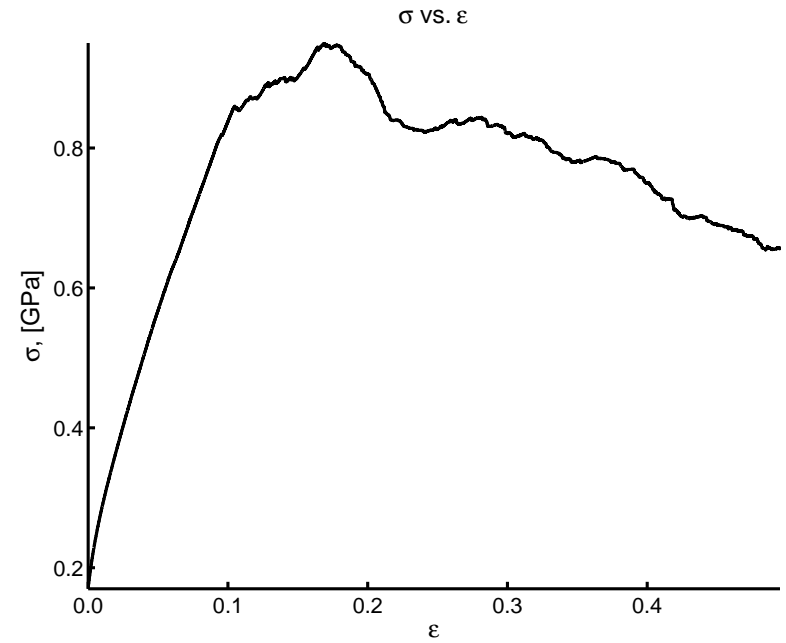

(b)

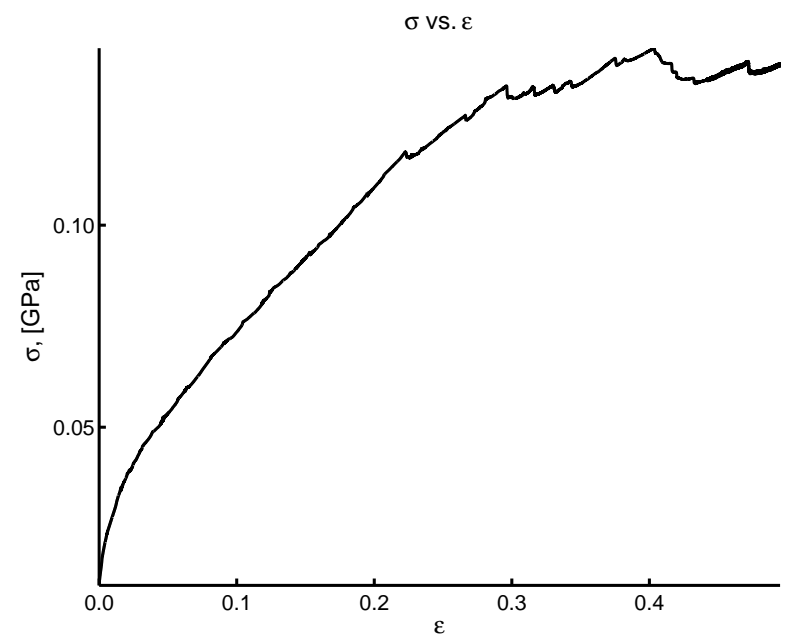

(d)

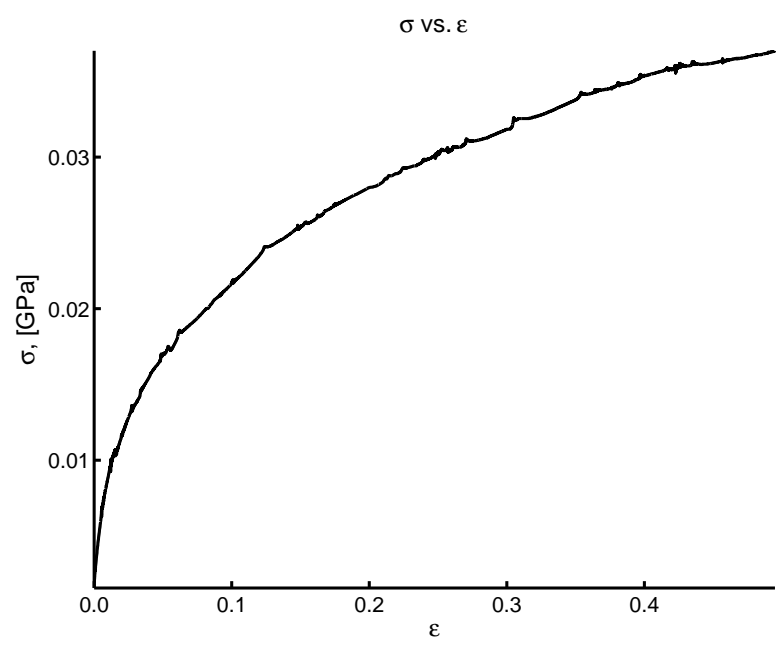

(f)

Figure C.4: Stress vs. Strain plots for silica aerogels and xerogels, $0.4 \mathrm{~mol} \% \mathrm{CH}_{3}$ bonded to Si. (a) $\rho=$ $1.49 \frac{g}{\mathrm{~cm}^{3}}$, (b) $\rho=1.05 \frac{g}{\mathrm{~cm}^{3}}$, (c) $\rho=0.58 \frac{g}{\mathrm{~cm}^{3}}$, (d) $\rho=0.45 \frac{g}{\mathrm{~cm}^{3}}$, (e) $\rho=0.35 \frac{g}{\mathrm{~cm}^{3}}$, (f) $\rho=0.28 \frac{\mathrm{g}}{\mathrm{cm}^{3}}$ 


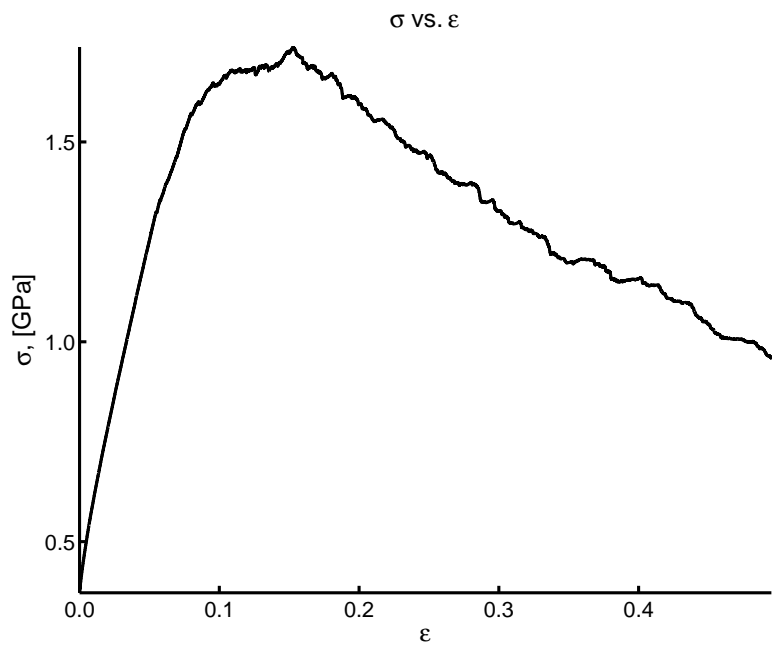

(a)

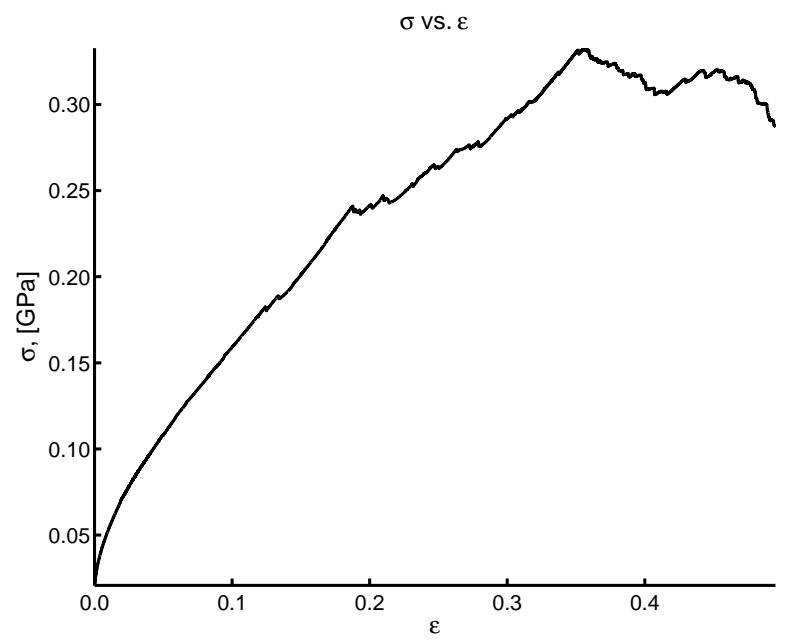

(c)

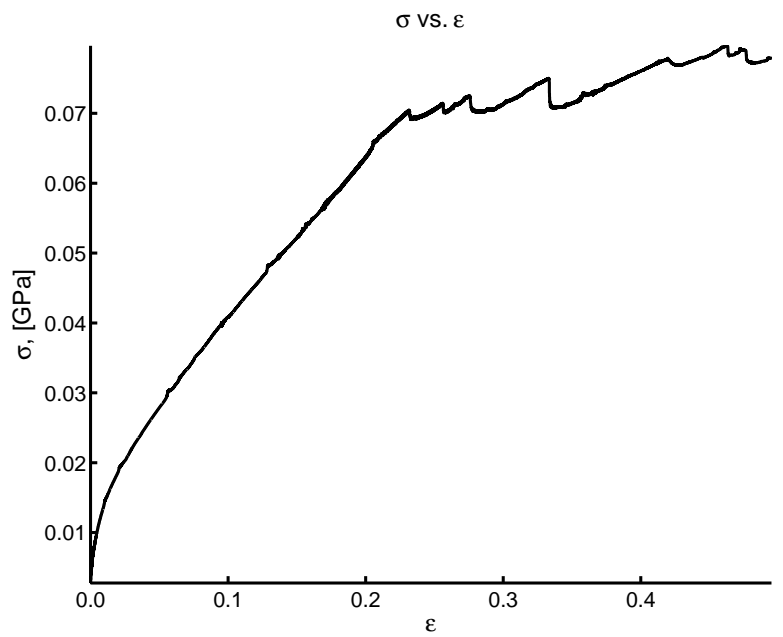

(e)

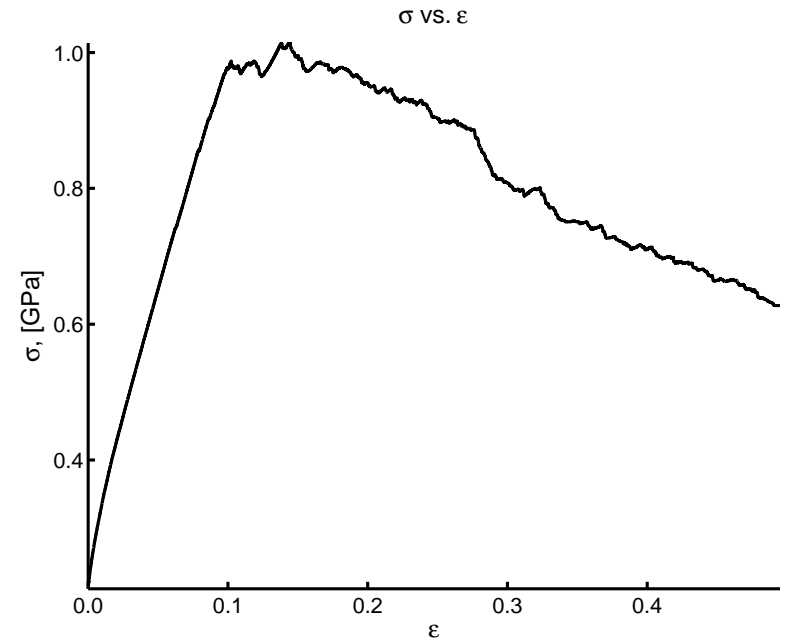

(b)

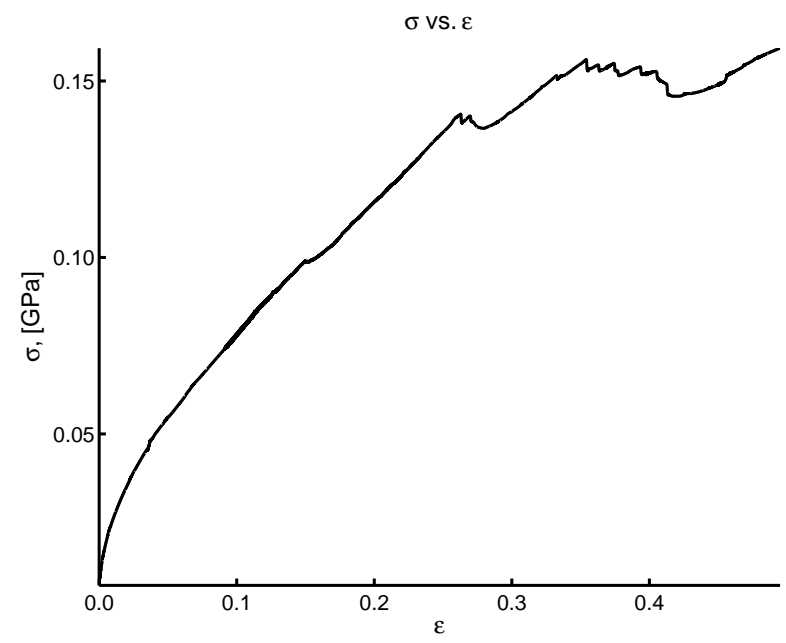

(d)

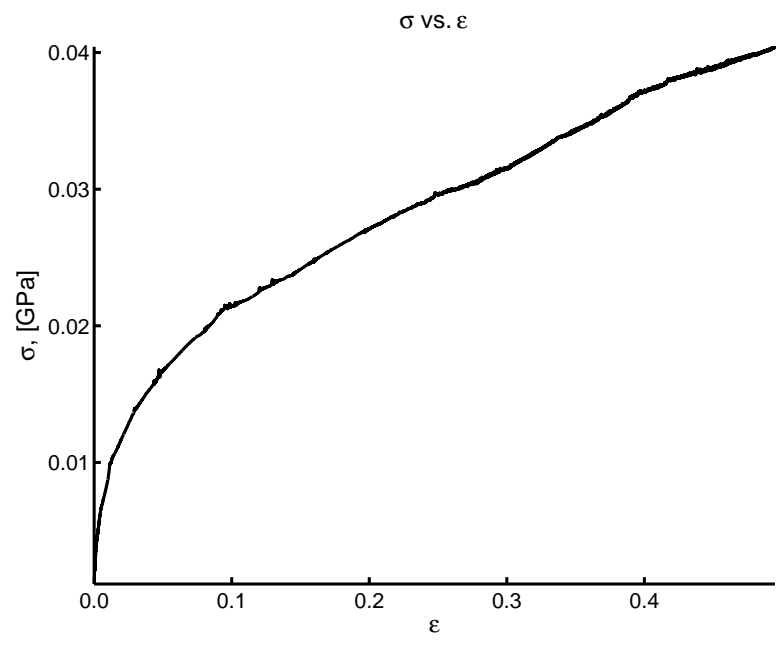

(f)

Figure C.5: Stress vs. Strain plots for silica aerogels and xerogels, $0.4 \mathrm{~mol} \% \mathrm{CH}_{3}$ bonded to O. (a) $\rho=$ $1.49 \frac{g}{\mathrm{~cm}^{3}}$, (b) $\rho=1.05 \frac{\mathrm{g}}{\mathrm{cm}^{3}}$, (c) $\rho=0.58 \frac{\mathrm{g}}{\mathrm{cm}^{3}}$, (d) $\rho=0.45 \frac{\mathrm{g}}{\mathrm{cm}^{3}}$, (e) $\rho=0.35 \frac{\mathrm{g}}{\mathrm{cm}^{3}}$, (f) $\rho=0.28 \frac{\mathrm{g}}{\mathrm{cm}^{3}}$ 